Florida International University FIU Digital Commons

3-30-1999

\title{
Tool cutting force modeling and wear estimation of micro-end-milling operations
}

Wei-yu Bao

Florida International University

DOI: $10.25148 /$ etd.FI14050423

Follow this and additional works at: https://digitalcommons.fiu.edu/etd

Part of the Mechanical Engineering Commons

\section{Recommended Citation}

Bao, Wei-yu, "Tool cutting force modeling and wear estimation of micro-end-milling operations" (1999). FIU Electronic Theses and Dissertations. 1386.

https://digitalcommons.fiu.edu/etd/1386

This work is brought to you for free and open access by the University Graduate School at FIU Digital Commons. It has been accepted for inclusion in FIU Electronic Theses and Dissertations by an authorized administrator of FIU Digital Commons. For more information, please contact dcc@fiu.edu. 
FLORIDA INTERNATIONAL UNIVERSITY

Miami, Florida

\section{TOOL CUTTING FORCE MODELING AND WEAR ESTIMATION OF MICRO-END-MILLING OPERATIONS}

A dissertation submitted in partial fulfillment of the requirements for the degree of DOCTOR OF PHILOSOPHY in

MECHANICAL ENGINEERING

by

Wei-Yu Bao 
To: Dean Gordon Hopkins

College of Engineering

This dissertation, written by Wei-Yu Bao, and entitled Tool Cutting Force Modeling and Wear Estimation of Micro End-Milling Operations, having been approved in respect to style and intellectual content, is referred to you for judgment.

We have read this dissertation and recommend that it be approved.

Sabri Tosunoglu

Tachung C \in'

Ibrahim N. Tansel, Major Professor

Data of Defense: March 30, 1999

The dissertation of Wei-Yu Bao is approved.

Dean Gordon Hopkins

College of Engineering

Dean Richard L. Campblell

Division of Graduate Studies

Florida International University, 1999 


\section{ACKNOWLEDGMENTS}

I would like to thank the members of the committee for their patience and constructive comments. I especially thank my major professor Dr. Ibrahim N. Tansel for introducing me to neural networks and genetic algorithms and showing me the beauty and joy that lie beyond the neuron and gene.

The majority of the experimental data was collected at Engineering Prototype Center of Radio Technology Division of Motorola Inc. I thank Mr. Bob Shisler, Chris Nelson, Derek Smith and Michael McCool of Motorola Inc. for their technical supports and recommendations. I also thank my colleagues Mr. Tacku T. Arkan and Mahendrakar Naudeshwar for their assistance and help. It would impossible to complete this research without their cooperation. 


\author{
ABSTRACT OF THE DISSERTATION \\ TOOL CUTTING FORCE MODELING AND WEAR ESTIMATION \\ OF MICRO END-MILLING OPERATIONS \\ by \\ Wei-Yu Bao \\ Florida International University, 1999 \\ Miami, Florida \\ Professor Ibrahim N. Tansel, Major Professor
}

The applications of micro-end-milling operations have increased recently. A Micro-End-Milling Operation Guide and Research Tool (MOGART) package has been developed for the study and monitoring of micro-end-milling operations. It includes an analytical cutting force model, neural network based data mapping and forecasting processes, and genetic algorithms based optimization routines. MOGART uses neural networks to estimate tool machinability and forecast tool wear from the experimental cutting force data, and genetic algorithms with the analytical model to monitor tool wear, breakage, run-out, cutting conditions from the cutting force profiles.

The performance of MOGART has been tested on the experimental data of over 800 experimental cases and very good agreement has been observed between the theoretical and experimental results. The MOGART package has been applied to the micro-end-milling operation study of Engineering Prototype Center of Radio Technology Division of Motorola Inc. 


\section{TABLE OF CONTENTS}

CHAPTER

PAGE

Chapter I Introduction

$\begin{array}{ll}\text { Chapter II Theoretical Background } & 4\end{array}$

2.1 Modeling of End Milling Operations 4

2.2 Neural Networks

2.3 Genetic Algorithms

2.4 Machinability and Monitoring of End Milling Operations

Chapter III Analytical Cutting Force Model of Micro-End-Milling Operations

3.1 Cutting Force Model without Tool Run-out

3.2 Cutting Force Model with Tool Run-out

3.3 Cutting Force Model of Conventional End Milling Operations

Chapter IV Model Based Cutting Force Characteristics and Surface Finish

4.1 Cutting Force Profiles

4.2 Cutting Force Characteristics

4.3 Work-piece Surface Roughness and Precision

5.1 Tool Breakage Detection

5.2 Tool Wear Estimation

5.3 Tool Run-out Estimation 
7.2 Representation of the Cutting Force Characteristics

7.3 Performance of Monitoring in Micro-End-Milling Operations

$\begin{array}{ll}\text { 8.1 Structure of MOGART Package } & 160\end{array}$

8.2 Analytical Cutting Force Model 164

8.3 Neural Network Research Tool 168

8.4 Genetic Algorithm Research Tool 175

8.5 Applications of Operation Guide in Micro-End-Milling Operations 178

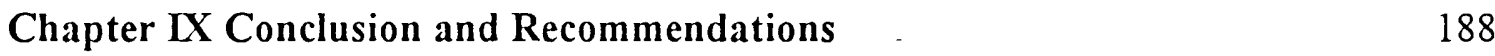

$\begin{array}{ll}\text { List of References } & 191\end{array}$

Vita

198 


\section{LIST OF TABLES}

TABLE

PAGE

Table 5.1 Results of the tool wear estimation model

Table 5.2 Results of the tool cutting angle identification

Table 6.1 Experimental contents

Table 6.2 Experimental equipment

Table 7.1 Maximum cutting force error of the analytical cutting force model

Table 7.2 Experimental data of 0.020 " diameter end mill machinability testing

Table 7.3 Experimental data of 0.0625 " diameter end mill machinability testing

Table 7.4 Results of the tool wear forecasting model testing

Table 7.5 Run-out experimental results of collet end mill holder

Table 7.6 Run-out experimental results of conventional end mill holder

Table 7.7 Results of the tool run-out estimation

Table 7.8 Results of the tool cutting condition monitoring 


\section{LIST OF FIGURES}

FIGURE

PAGE

Figure 2.1 Construction of neural networks

Figure 2.2 Construction of the three-layer neural networks

Figure 2.3 A research area map with the ground-water contamination data

Figure 2.4 Estimation of the ground-water contamination distributions

Figure 2.5 Computational results of a mapping study

Figure 2.6 Estimated results of a mapping study

Figure 2.7 Results of square wave forecasting

Figure 2.8 Results of function forecasting

Figure 2.9 A data chromosome with 16 genes presented three DNA

Figure 2.10 Biological evolution cycle of species

Figure 2.11 A mating procedure of genetic algorithms

Figure 2.12 Fixed-point crossover genetic procedure

Figure 2.13 Uniform crossover genetic procedure

Figure 2.14 Jumping mutation genetic procedure

Figure 2.15 Genetic evolution procedure of the tool cutting angle monitoring

Figure 2.16 Function of an optimization case

Figure 2.17 Genetic evolution procedure of the function optimization

Figure 3.1 Tool cutting edge profiles of micro-end-milling operations

Figure 3.2 Tool cutting edge profiles of Tlusty's cutting force model 
Figure 4.1 Cutting force of two-flute tool, climbing milling without tool run-out

Figure 4.2 Cutting force of two-flute tool, conventional milling without tool run-out

Figure 4.3 Cutting force of two-flute tool, climbing milling with tool run-out

Figure 4.4 Cutting force of two-flute tool, conventional milling with tool run-out

Figure 4.5 Cutting force of four-flute tool, climbing milling without tool run-out

Figure 4.6 Cutting force of four-flute tool, conventional milling without tool run-out

Figure 4.7 Cutting force characteristics with spindle speed

Figure 4.8 Cutting force characteristics with spindle speed

Figure 4.9 Cutting force characteristics with feed rate

Figure 4.10 Cutting force characteristics with depth of cut

Figure 4.11 2-D cutting force characteristics with spindle speed and feed rate

Figure 4.12 3-D cutting force characteristics with spindle speed and feed rate

Figure 4.13 2-D cutting force characteristics with spindle speed and depth of cut

Figure 4.14 3-D cutting force characteristics with spindle speed and depth of cut

Figure 4.15 2-D cutting force characteristics with feed rate and depth of cut

Figure 4.16 3-D cutting force characteristics with feed rate and depth of cut

Figure 4.17 Cutting force characteristics with tool run-out

Figure 4.18 Cutting force characteristics with tool run-out angle

Figure 4.19 2-D cutting force characteristics with tool run-out and its angle

Figure 4.20 3-D cutting force characteristics with tool run-out and its angle

Figure 4.21 Cutting force characteristics with entry cutting angle

Figure 4.22 Cutting force characteristics with tool exit cutting angle 
Figure 4.23 Cutting force characteristics with tool diameter and spindle speed

Figure 4.24 Cutting force characteristics with tool diameter and feed rate

Figure 4.25 Cutting force characteristics with tool diameter and depth of cut

Figure 4.26 2-D cutting force characteristics with tool diameter and spindle speed

Figure 4.27 3-D cutting force characteristics with tool diameter and spindle speed

Figure 4.28 2-D cutting force characteristics with tool diameter and feed rate

Figure 4.29 3-D cutting force characteristics with tool diameter and feed rate

Figure 4.30 2-D cutting force characteristics with tool diameter and depth of cut

Figure 4.31 3-D cutting force characteristics with tool diameter and depth of cut

Figure 4.32 Cutting force characteristics with toot flute numbers

Figure 4.33 Cutting force characteristics with toot helix angle

Figure 4.34 Work-piece surface roughness by considering the tool cutting edge tip profiles

Figure 4.35 Work-piece surface precision by considering the tool run-out

Figure 5.1 AE activity of micro-end-milling experiment I, case 1

Figure 5.2 AE activity of micro-end-milling experiment I, case 2

Figure 5.3 AE activity of micro-end-milling experiment II, case 1

Figure 5.4 AE activity of micro-end-milling experiment II, case 2

Figure 5.5 Tool cutting force of micro-end-milling experiment I

Figure 5.6 Tool cutting force of micro-end-milling experiment II

Figure 5.7 New tool cutting force average of experiment I

Figure 5.8 Tool cutting force average before breakage of experiment I 
Figure 5.9 New tool cutting force variation of experiment I

Figure 5.10 Tool cutting force variation before breakage of experiment I

Figure 5.11 New tool cutting force average of experiment II

Figure 5.12 Tool cutting force average before breakage of experiment II

Figure 5.13 New tool cutting force variation of experiment II

Figure 5.14 Tool cutting force variation before breakage of experiment II

Figure 5.15 Monitoring of tool wear by the thrust direction cutting force

Figure 5.16 Monitoring of tool wear by the feed direction cutting force

Figure 5.17 Indirect monitoring of tool wear by the cutting forces

Figure 5.18 Genetic evolution procedure of the tool wear model

Figure 5.19 An empirical tool wear model

Figure 5.20 Comparison of the tool wear model

Figure 5.21 Tool run-out estimation procedure

Figure 5.22 Cutting force of climbing milling with tool run-out

Figure 5.23 Genetic evolution procedure of tool run-out estimation

Figure 5.24 Tool cutting angles of end milling operations

Figure 5.25 Tool cutting angle identification procedure

Figure 5.26 Spindle speed identification

Figure 5.27 Tool cutting condition identification procedure

Figure 5.28 Tool optimal working condition estimation procedure

Figure 6.1 Experimental setup 
Figure 6.4 Data acquisition

Figure 6.5 Hardness measurement

Figure 6.6 Image processing system

Figure 6.7 The computer screen showing the software used for Image processing

Figure 6.8 The cutting edges of a new tool

Figure 6.9 The cutting edges of a worn tool

Figure 6.10 Work-piece surface cut by a new tool

Figure 6.11 Work-piece surface cut by a worn tool

Figure 7.1 Experimental cutting forces of micro-end-milling without run-out

Figure 7.2 Analytical model based cutting forces of micro-end-milling without run-out 137

Figure 7.3 Experimental cutting forces of micro-end-milling with run-out

Figure 7.4 Analytical model based cutting forces of micro-end-milling with run-out

Figure 7.5 Thrust direction maximum cutting force of 0.020 " diameter end mills

Figure 7.6 Feed direction maximum cutting force of 0.020 " diameter end mills

Figure 7.7 Thrust direction maximum cutting force of 0.0625 " diameter end mills

Figure 7.8 Feed direction maximum cutting force of 0.0625 " diameter end mills

Figure 7.9 Feed direction maximum cutting force of 0.020 " diameter end mills

Figure 7.10 Feed direction maximum cutting force of 0.030 " diameter end mills

Figure 7.11 Feed direction maximum cutting force of 0.050 " diameter end mills

Figure 7.12 Feed direction maximum cutting force of 0.0625 " diameter end mills

Figure 7.13 Model based thrust direction maximum cutting force of micro-end-milling 147 
Figure 7.14 Model based feed direction maximum cutting force of micro-end-milling 147

$\begin{array}{ll}\text { Figure 7.15 Difference between micro and conventional end milling operations } & 148\end{array}$

Figure 7.16 Tool breakage monitoring by AE activity of experiment II, case $1 \quad 151$

Figure 7.17 Tool breakage monitoring by AE activity of experiment II, case $2 \quad 151$

$\begin{array}{ll}\text { Figure 7.18 Performance of the tool wear forecast model } & 153\end{array}$

$\begin{array}{ll}\text { Figure 7.19 Thrust direction cutting force of the new tool } & 155\end{array}$

Figure 7.20 Thrust direction cutting force of the tool before breakage 155

$\begin{array}{lr}\text { Figure 8.1 MOGART program } & 159\end{array}$

$\begin{array}{ll}\text { Figure 8.2 Diagram of MOGAT program } & 161\end{array}$

$\begin{array}{ll}\text { Figure } 8.3 \text { Menu of MOGART program } & 163\end{array}$

$\begin{array}{ll}\text { Figure } 8.4 \text { Cutting force estimation } & 165\end{array}$

$\begin{array}{ll}\text { Figure } 8.5 \text { Cutting force report } & 165\end{array}$

$\begin{array}{ll}\text { Figure 8.6 Cutting force profile } & 167\end{array}$

$\begin{array}{ll}\text { Figure } 8.7 \text { Tool cutter profile } & 167\end{array}$

$\begin{array}{lr}\text { Figure } 8.8 \text { Neural network project } & 169\end{array}$

$\begin{array}{ll}\text { Figure 8.9 Neural network setup } & 169\end{array}$

$\begin{array}{ll}\text { Figure 8.10 Training data input } & 170\end{array}$

$\begin{array}{ll}\text { Figure 8.11 Neural network running } & 172\end{array}$

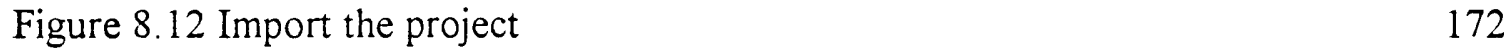

$\begin{array}{ll}\text { Figure 8.13 Neural network testing report } & 173\end{array}$

$\begin{array}{ll}\text { Figure 8.14 Graphics of neural network testing results } & 174\end{array}$

$\begin{array}{ll}\text { Figure } 8.15 \text { GATool setup step } 1 & 176\end{array}$ 
Figure 8.16 GATool setup step 2

Figure 8.17 GATool results

Figure 8.18 Characteristics of maximum cutting forces

Figure 8.19 Three-dimensional cutting force graphic

Figure 8.20 Work-piece surface precision estimation

Figure 8.21 Work-piece surface roughness estimation

Figure 8.22 Tool run-out estimation

Figure 8.23 Tool life estimation

Figure 8.24 Optimal working condition selection 


$$
\begin{aligned}
& \mathrm{r} \quad=\text { tool radius (inch) } \\
& \mathrm{Z} \quad=\text { the numbers of tool teeth } \\
& z \quad=\text { the ordinal number of tool teeth } \\
& \beta \quad=\text { tooth helix angle (rad) } \\
& \mathrm{n} \quad=\text { spindle speed }(\mathrm{rpm}) \\
& \omega \quad=\text { spindle circle speed }(1 / \mathrm{sec} .) \\
& \mathrm{f} \quad=\text { feed rate }(\mathrm{ipm}) \\
& f_{\mathrm{t}} \quad=\text { feed per tooth (inch) } \\
& \mathrm{t}=\text { time }(\mathrm{sec} .) \\
& \text { a } \quad=\text { depth of thrust (inch) } \\
& \text { b = depth of cut (inch) } \\
& \theta \quad=\text { tool cutting angle }(\mathrm{rad}) \\
& \lambda \quad=\text { leading angle (rad) } \\
& \alpha \quad=\text { engagement angle (rad) } \\
& \varphi \quad=\text { cutting angle of work piece (rad) } \\
& \psi \quad=\text { tool cutter angle per tooth (rad) } \\
& \delta=\text { computing angle }(\mathrm{rad}) \\
& \mathrm{f}_{\mathrm{c}} \quad=\text { computing feed (inch) } \\
& \mathrm{h}=\text { cutting chip thickness (inch) }
\end{aligned}
$$


$\mathrm{H}$ = not working cutter edge length (inch)

$\mathrm{p} \quad=$ proportional factor

$\mathrm{K}_{\mathrm{m}} \quad=$ material coefficient $\left(\mathrm{N} / \mathrm{cm}^{2}\right)$

$\mathrm{K}_{\mathrm{w}} \quad=$ wear coefficient

$F_{u} \quad=$ unit force $(N)$

$\mathrm{F}_{\mathrm{t}} \quad=$ tangential cutting force $(\mathrm{N})$

$\mathrm{F}_{\mathrm{r}} \quad$ = radial cutting force $(\mathrm{N})$

$\mathrm{F}_{\mathrm{x}} \quad=$ feed direction cutting force $(\mathrm{N})$

$\mathrm{F}_{\mathrm{y}} \quad=$ thrust direction cutting force $(\mathrm{N})$

$r_{0} \quad=$ run-out length (inch)

$\gamma \quad=$ run-out angle (rad)

$\theta_{\mathbf{s}} \quad=$ integrating start angle (rad)

$\theta_{\mathrm{e}} \quad=$ integrating end angle (rad)

$\mathrm{S}_{\mathrm{r}} \quad$ = work-piece surface roughness (inch)

$\mathrm{S}_{\mathrm{p}} \quad$ = work-piece surface precision (inch) 


\section{Chapter I}

\section{Introduction}

Micro-end-milling operations were first used for manufacturing of special purpose equipment in biomedical and aerospace applications. However, miniaturization of many consumer products and esthetic goals drastically increased micro-end-milling operations in the conventional shop floor. Currently, many state-of-the-art consumer product manufacturers widely use micro-tools with less than $2 \mathrm{~mm}$ diameter to prepare the plastic injection molds of their parts.

At the first glance, micro-end-milling operations look like conventional end-milling operations with only dimensional difference. However, it was found that cutting force characteristics of micro-end-milling operations were different from those of conventional end milling operations after their machinability tests had been done. Micro-tools have very short tool life compare to the conventional tools. If the cutting conditions are not selected properly, micro-tools will be broken in a few seconds. Depending on the hardness of work-pieces, even in the identical cutting conditions micro-tools may have less than 10 " of tool life. Operators have to carefully select the cutting conditions with the small margin of errors and monitor the machining operations since those tools will create unnoticeable sound and vibration. Because of their tiny size, it is very different to detect their breakage. Many hours of machining time could be wasted if the tool failure is not detected on time. In addition, the ratio of the tool run-out to tool diameter becomes very big compare to 
conventional tools. In many cases, micro-tools are subjected to larger cutting forces since only half of the cutting edges remove the material from the work-piece.

Even though the need for a concentrated study on micro-machining is known, very limited studies had been completed. In this dissertation, a new analytical model was developed for micro-end-milling operations, and a series of techniques were developed to use this model for machinability study, cutting condition monitoring, run-out estimation, tool wear modeling and breakage detection.

All the developed tools were integrated in a single software package. The package effectively uses neural networks and genetic algorithms together with the analytical model. In this work, the following studies are integrated:

- A new analytical cutting force model for micro-end-milling with or without tool run-out.

- Tool cutting force variation estimators based on the analytical model and neural networks mapping.

- Tool wear estimation by using the genetic algorithms and neural networks.

- Tool breakage detection method.

- Tool cutting condition monitoring method by using the analytical model and genetic algorithms.

- Run-out estimation by detecting the cutting force profiles.

- The analytical model based surface finish calculation.

The developed package is capable to help engineers to select the optimal cutting conditions with minimal experiments, to evaluate the performance of their operations and 
to monitor the tool condition. The cost of micro-machining operations, setup time and number of inspections could be reduced by using the package effectively.

To verify the analytical cutting force model and get the necessary data, more than 800 experiments have been performed for the cooperation of Mechatronics Lab of Mechanical Engineering Department of Florida International University and Engineering Prototype Center of Radio Technology Division of Motorola Inc.

The theoretical background of the studies is introduced in Chapter II, which includes the tool cutting force modeling of conventional end milling operations, neural networks and genetic algorithms. The derivation of the developed analytical cutting force model of the micro-end-milling operations is presented in Chapter III. The analytical model based cutting force characteristics of the micro-end-milling operations are discussed in Chapter IV. The analytical model based the monitoring methods of the micro-endmilling operations are proposed in Chapter $\mathrm{V}$, which include tool breakage, wear, run-out and cutting conditions. The Chapter VI presents the experiment setup and coverage. The results of the studies are discussed in Chapter VII. Chapter VIII is a user guide of the Micro-End-Milling Operation Guide and Research Tools (MOGART) program. The conclusion of the researches and recommended future work are presented in Chapter IX. 


\section{Chapter II}

\section{Theoretical Background}

In this chapter, the theoretical background of the existed modeling and processing techniques that were used in the dissertation are outlined. First the modeling of end milling operations is discussed. This model will be modified for micro-end-milling operations in the next chapter. For data processing, neural networks and genetic algorithms are presented. The neural networks are used for mapping, classification and forecasting. The genetic algorithms are used for optimization, modeling and monitoring.

\subsection{Modeling of End Milling Operations}

\section{Tlusty's Cutting Force Model of End Milling Operations}

In $1975, \mathrm{~J}$. Tlusty developed an analytical cutting force model of the end milling operations to calculate the cutting force variations. ${ }^{[1]}$ Tlusty's cutting force model was developed based on the following three assumptions:

Assumption 1: The tangential cutting force is proportional to the cutting areas:

$$
\mathrm{F}_{\mathrm{t}}=\mathrm{K}_{\mathrm{m}} \mathrm{bh}
$$

Assumption 2: The radial cutting force is proportional to the tangential cutting force:

$$
F_{r}=p F_{t}
$$

Assumption 3: The chip thickness can be expressed with the following expression:

$$
\mathrm{h}=\mathrm{f}_{\mathrm{t}} \sin \theta
$$


In the first assumption, the cutting chip thickness $h$ is not a constant, but a function of $z$ because of tool helix angle. The formula 2.1.1 can be rewritten as:

$\mathrm{dF}_{\mathrm{t}}=\mathrm{K}_{\mathrm{m}} \mathrm{h}(\mathrm{z}) \mathrm{dz}$

Because of $z=z(\theta)$ and $d z=\frac{r}{\tan \beta} d \theta$, it becomes:

$d F_{t}=2\left(F_{u} / f_{t}\right) h(\theta) d \theta$

$d F_{r}=p d F_{t}=2\left(F_{u} / f_{t}\right) p h(\theta) d \theta$

where: $F_{u}=\frac{K_{m} r f_{t}}{2 \tan \beta}$

The tool cutting forces of the feed direction $\mathrm{x}$ and the thrust direction $\mathrm{y}$ are calculated.

$$
\begin{aligned}
& d F_{x}=-d F_{t} \cos \theta-d F_{r} \sin \theta=-2\left(F_{u} / f_{t}\right) h(\theta)(\cos \theta d \theta+p \sin \theta d \theta) \\
& d F_{y}=d F_{t} \sin \theta-d F_{r} \cos \theta=2\left(F_{u} / f_{t}\right) h(\theta)(\sin \theta d \theta-p \cos \theta d \theta)
\end{aligned}
$$

Considering the third assumption, the formulas 2.1.6 and 2.1.7 become:

$$
\begin{aligned}
& d F_{x}=-2 F_{u}\left(\sin \theta \cos \theta d \theta+p \sin ^{2} \theta d \theta\right) \\
& d F_{y}=2 F_{u}\left(\sin ^{2} \theta d \theta-p \sin \theta \cos \theta d \theta\right)
\end{aligned}
$$

After integration, the analytical cutting force model has been derived.

$\underline{F_{\underline{z}}}=-F_{\underline{\underline{u}}}\left[p\left(\theta_{\underline{e}}-\theta_{\underline{s}}\right)+\left(\sin ^{2} \theta_{\underline{e}}-\sin ^{2} \theta_{\underline{\underline{S}}}\right)-0.5 p\left(\sin 2 \theta_{\underline{e}}-\sin 2 \theta_{\underline{\underline{S}}}\right)\right]$

$\underline{F}_{Y}=F_{\underline{u}}\left[\left(\theta_{\underline{e}}-\theta_{s}\right)-p\left(\sin ^{2} \theta_{\underline{e}}-\sin ^{2} \theta_{s}\right)-0.5\left(\sin 2 \theta_{\underline{e}}-\sin 2 \theta_{s}\right)\right]$

The resultant cutting force on the $\mathrm{x}-\mathrm{y}$ plant is:

$\mathrm{F}_{\mathrm{r}}^{2}=\mathrm{F}_{\mathrm{x}}^{2}+\mathrm{F}_{\mathrm{y}}^{2}$ 
To calculate the tool cutting force using the formulas 2.1 .10 and 2.1 .11 , three parameters have to be considered.

The first parameter is the tool cutter angle per tooth $\psi$.

$$
\psi=\frac{2 \pi}{Z}
$$

The second parameter is the cutting angle of the work-piece $\varphi$.

$$
\varphi=\arccos \left(\frac{r-a}{r}\right)
$$

The third parameter is the engagement angle $\alpha$.

$$
\alpha=\frac{b \tan \beta}{r}
$$

Three different machining operations have been discussed in Tlusty's model.

Case 1: $\alpha \leq \varphi$ and $\alpha+\varphi \leq \psi$

For conventional milling operations:

$$
\begin{array}{llll}
\text { section 1: } & {[0, \alpha]} & \theta_{\mathrm{s}}=0 & \theta_{\mathrm{e}}=\theta \\
\text { section 2: } & {[\alpha, \varphi]} & \theta_{\mathrm{s}}=\theta-\alpha & \theta_{\mathrm{e}}=\theta \\
\text { section 3: } & {[\varphi, \varphi+\alpha]} & \theta_{\mathrm{s}}=\theta-\alpha & \theta_{\mathrm{e}}=\varphi
\end{array}
$$

For climbing milling operations:

$$
\begin{array}{llll}
\text { section 1: } & {[\pi-\varphi, \pi-\varphi+\alpha]} & \theta_{s}=\pi-\varphi & \theta_{\mathrm{e}}=\theta \\
\text { section 2: } & {[\pi-\varphi+\alpha, \pi]} & \theta_{s}=\theta-\alpha & \theta_{\mathrm{e}}=\theta \\
\text { section 3: } & {[\pi, \pi+\alpha]} & \theta_{s}=\theta-\alpha & \theta_{\mathrm{e}}=\pi
\end{array}
$$

Case 2: $\alpha \geq \varphi$ and $\alpha+\varphi \leq \psi$

For conventional milling operations: 
section 1: $[0, \varphi] \quad \theta_{\mathrm{s}}=0 \quad \theta_{\mathrm{e}}=\theta$

section 2: $[\varphi, \alpha] \quad \theta_{\mathrm{s}}=0 \quad \theta_{\mathrm{e}}=\varphi$

section 3: $\quad[\alpha, \varphi+\alpha] \quad \theta_{\mathrm{s}}=\theta-\alpha \quad \theta_{\mathrm{e}}=\varphi$

For climbing milling operations:

section 1: $\quad[\pi-\varphi, \pi] \quad \theta_{\mathrm{s}}=\pi-\varphi \quad \theta_{\mathrm{e}}=\theta$

section 2: $[\pi, \alpha] \quad \theta_{\mathrm{s}}=\pi-\varphi \quad \theta_{\mathrm{e}}=\pi$

section 3: $\quad[\alpha, \pi+\alpha] \quad \theta_{\mathrm{s}}=\theta-\alpha \quad \theta_{\mathrm{e}}=\pi$

Case 3: $\alpha+\varphi \geq \psi$

Because of overlapping, the tool cutting force of the overlapped part is equal to the sum of the cutting forces of both cutting edges.

Tlusty's cutting force model has been widely used. It has reasonable assumptions, straightforward derivation and can be easily applied to most of conventional end milling operations without tool run-out.

In micro-end-milling operations, the tool diameter is very small. The micro-tools are easily worn and suddenly broken. The influence of the tool run-out becomes significant to the cutting force variation because of their tiny sizes. Tlusty's model didn't consider the tool run-out and wear. It also didn't explain the difference between the conventional and climbing milling operations. In micro-end-milling operations the ratio of the feed per tooth to the tool radius $\left(\mathrm{f}_{\mathrm{l}} / \mathrm{r}\right)$ usually can not be neglected. In this case the third assumption of Tlusty's model is not valid. A new analytical cutting force model of micro-end-milling has been looked for since the micro-end-mills were applied to the manufacturing. 


\section{Improvements of the Analytical Cutting Force Model.}

To include tool run-out into the model, researchers used two approaches. The first approach was development of a new analytical model. Gygas improved Trusty's cutting force modal by considering different total cutting angle, climbing and conventional milling, symmetric and asymmetric cut in $1979 .{ }^{[2]}$ Based on Tlusty's three assumptions and experimental data, another empirical cutting force modal was developed by Yucesan et al., in $1990 .{ }^{[3]}$ The different cutting conditions were investigated and analyzed by statistical methods and plasticity theory. The empirical model considered the cutting force coefficients of Tlusty's first assumption as a function of the chip thickness and also gave the limitations of integration angles. Investigating the tool vibration in three-dimension cutting, Jemielniak derived a formula from steady state cutting to determine the dynamic cutting coefficients in $1992 .{ }^{[4]}$ To improve Tlusty's cutting force model with tools run-out and keep the analysis simplicity, Wang et al. developed a cutting force model in frequency domain in $1994 .{ }^{[5]}$ The model was derived as the convolution of three component functions, and the effect of cutter run-out was taken into consideration in forming revised chip thickness and average chip thickness expressions. Another milling operation with runout model was developed by Gu et al. in $1991 .{ }^{[6]}$ The run-out were considered as two new items into Tlusty's third assumption and estimated by cutting force signal.

The second approach was to develop a computational cutting force model. Sutherland and DeVor developed a computational model in which the chip load and runout were considered in $1986 .{ }^{[7]}$ Armarego and Deshpande focused on the eccentricity and 
deflection of the cutter, developed three-component based cutting models for end-milling force, torque and power predictions. These model can be used to predict the average and fluctuating force components and torque in $1991 .{ }^{[8]}$ The deflection of the end-mill and the work-piece, and surface error were predicted by using the model rather than the rigid endmilling system. Kim and Ehmann described a procedure for the three-dimensional static and dynamic cutting force simulation in face milling in $1993{ }^{[9]}$ The cutting forces created by machine tool vibrations was simulated in the model.

Tlusty's model has been improved in many different ways. Most developed models depended on the tlusty's three assumptions. None of the previous studies discussed or proved the tlusty's third assumption in theory and derived a model with tool run-out by directly considering the cutting chip thickness. In this dissertation, a new analytical cutting force model is derived from the tool cutting edges and their tip profiles equations. The model represents micro-end-milling and conventional end milling operations without or with tool run-out. It also considers tool wear. The model proves the existence of the Tlusty's third assumptions in theory and explains the difference between micro-end-milling and conventional end milling operations. The derivation and discussion of the new model will be presented in the next chapter. 


\subsection{Neural Networks}

\section{Basic Theory of Neural Networks}

Neural networks are a class of dynamic computational models that mimic the constructions and operations of a biological brain to react the real world problems. The first successful neural network was developed during 1957 and 1958 by Frank Rosenblatt, Charles Wightman and others ${ }^{[10]}$. The basic idea was to construct a network by using some nodes (neurons) connected together by some connection channels (nerves), which was able to carry and process the information through its input and output interfaces (see Figure 2.1). For different problems, only the connection weights between the nodes of the network had to be adjusted by a training procedure.

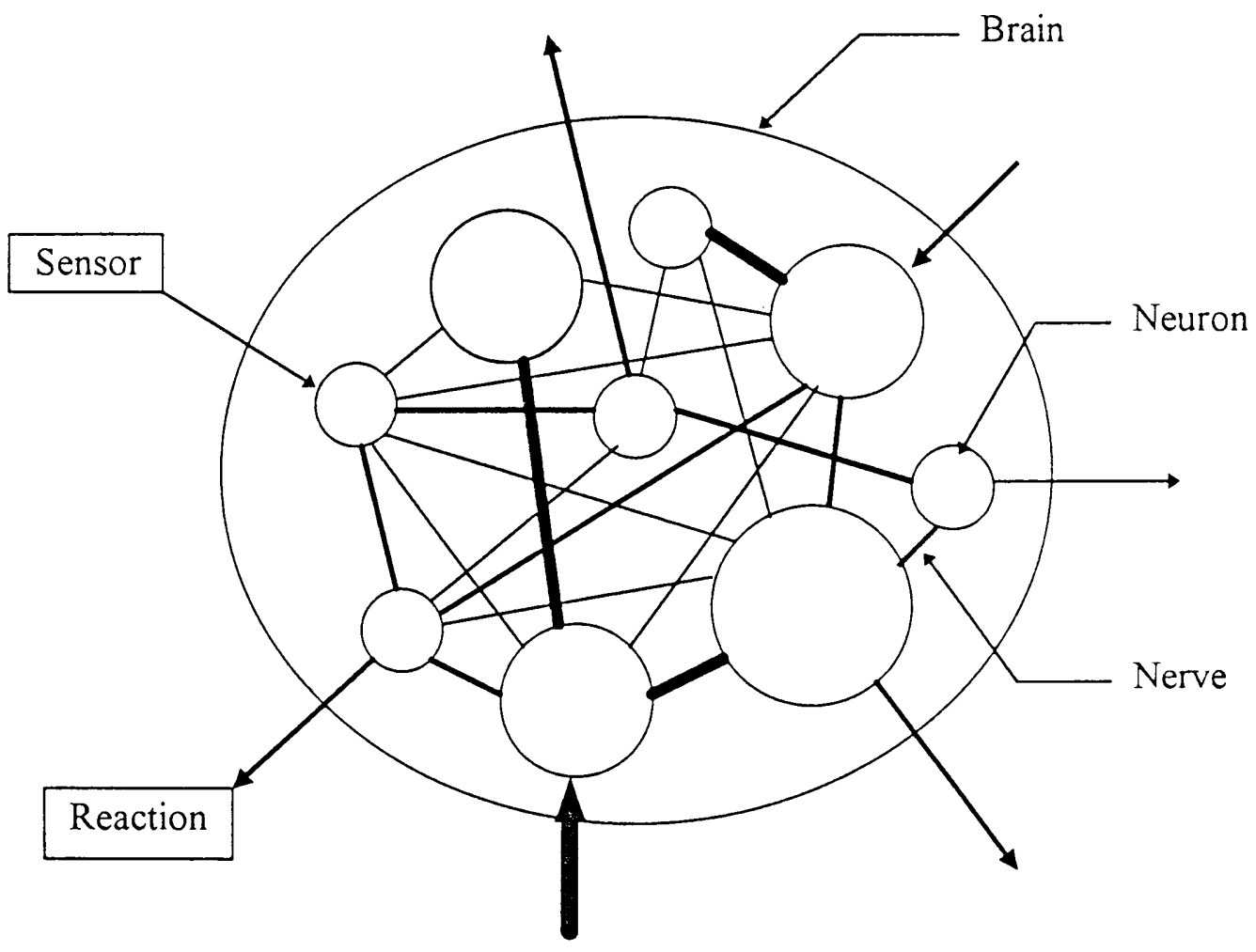

Figure 2.1 Construction of neural networks 
A basic theory of neural networks was contributed by mathematician Andrei Kolmogorov in $1957 .{ }^{[11]}$ He not only proved the existence of the neural networks, but also commented that they could be trained.

Theorem 1: (Kolmogorov's Neural Network Existence Theorem) Given any continuous function $\mathrm{f}:[0,1]^{\mathrm{n}} \rightarrow \mathbf{R}^{\mathbf{m}}, \mathrm{f}(\mathbf{x})=\mathbf{y}, \mathrm{f}$ can be implemented exactly by a three-layer feedforward neural network having $\mathrm{n}$ fan-out processing elements in the first ( $\mathbf{x}$ - input) layer, $(2 n+1)$ processing elements in the middle layer, and m processing elements in the top $(y-$ output) layer.

Theorem 2: Given any $\varepsilon>0$ and any $L_{2}$ function $f:[0,1]^{n} \rightarrow \mathbf{R}^{m}$, there exists a threelayer back-propagation neural network that can approximate $f$ to within $\varepsilon$ mean squared error accuracy.

The Kolmogorov's existence theorem proved that neural networks with three layers were able to implement an arbitrary function. The Kolmogorov's second theorem proved that neural networks were able to implement the function in any accuracy by adjusting the connection weights.

\section{Architecture of the Back-propagation Neural Network}

The back-propagation neural network is one of the most important historical developments in the neural networks. It is a powerful tool to solve mapping, classification and forecasting problems, which has been proved in many applications. In the designs of the back-propagation neural network, three layers (input layer, hidden layer and output layer) are constructed according to Kolmogorov's existence theorem. The information of 
distances and directions between the actual and estimating output data is used to update the connection weights among the connected nodes layer by layer through a backpropagation. A construction of the back-propagation neural network is presented in Figure 2.2.

Output layer:

Hidden layer:

Input layer:

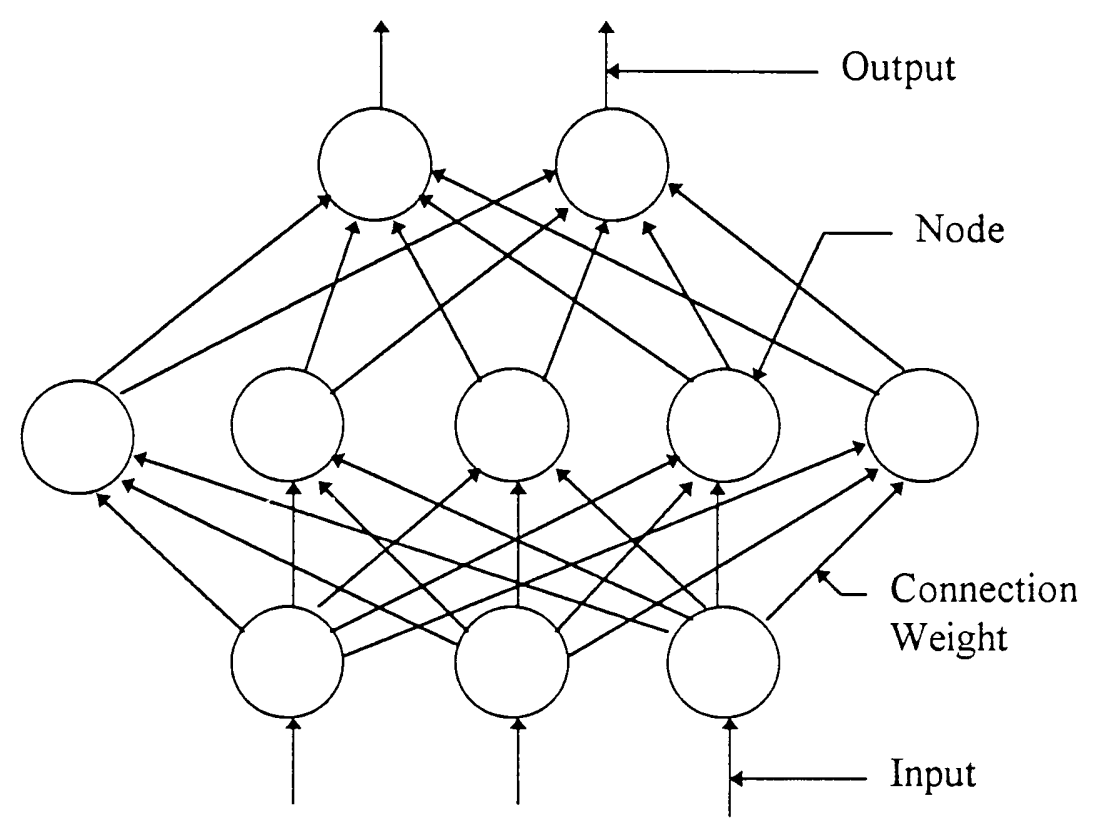

Figure 2.2 Construction of the three-layer neural networks

The back-propagation neural network could be trained by following three steps.

Step 1: Estimate output data by using forward-propagation calculations.

$$
\begin{aligned}
& \mathbf{h}=\mathbf{w}_{1} \delta(\mathbf{x}) \\
& \mathbf{y}_{\mathrm{e}}=\mathbf{w}_{2} \delta(\mathbf{h})
\end{aligned}
$$

where: $\mathbf{x}, \mathbf{h}$ and $\mathbf{y}_{\mathrm{e}}$ are the data of the input, hidden and output layers.

$\delta(\mathbf{x})$ and $\delta(\mathbf{h})$ are the unitizing transfers of the input and hidden layer data.

$\mathbf{w}_{1}$ and $\mathbf{w}_{2}$ are the connection weights between input and hidden nodes, hidden and output nodes. In the beginning, they are set randomly. 
Step 2: Calculate the error (distance) between the actual and estimated data (points).

$\varepsilon=\left|\mathbf{y}-\mathbf{y}_{\mathrm{e}}\right|$

where: $\mathbf{y}$ and $\mathbf{y}_{\mathrm{e}}$ are the actual and estimated data.

Step 3: Find the direction of the actual point, and update the weights by using the backpropagation calculations.

$$
\begin{aligned}
& \mathbf{w}_{2}^{\text {new }}=\mathbf{w}_{2}^{\text {old }}-\alpha \mathbf{f}_{1}\left(\varepsilon, \mathbf{y}_{\mathrm{e}}\right) \\
& \mathbf{w}_{1}^{\text {new }}=\mathbf{w}_{1}^{\text {old }}-\alpha \mathbf{f}_{1}(\varepsilon, \mathbf{h}) \\
& \text { where: } \alpha \text { is learning rate. }
\end{aligned}
$$

Repeat the three steps until the error $\varepsilon$ is less than the requested error level.

\section{Applications of the Back-propagation Neural Network}

The back-propagation neural network can be applied to solve the time independent problems called mapping and time series problems called forecasting. A Neural Network Tool program (NNTool) developed in 1995 and modified in 1996. ${ }^{[12][13]}$ is an application of back-propagation neural networks. It has been successfully applied to determine the underground contamination of New York area ${ }^{[14]}$ and Miami International Airport area. ${ }^{[15]}$ The three-dimensional underground contamination distribution graphics of both areas were generated by using 131 and 49 sample data sets respectively. In the research of micro-end-milling operations, it has been used to estimate the maximum cutting force of the micro-end-milling operations with different selected working conditions and tool parameters by using a few experimental data ${ }^{[16]}$ (see Chapter V). 
Mapping: The problem addressed by mapping is the approximate implementation of a function $\mathrm{f}: \mathrm{A} \subset \mathbf{R}^{\mathrm{n}} \rightarrow \mathbf{R}^{\mathrm{m}}$, from a bounded subset $\mathrm{A}$ of $\mathrm{n}$-dimensional Euclidean space to a bounded subset $\mathrm{f}[\mathrm{A}]$ of $\mathrm{m}$-dimensional Euclidean space, by training on the example cases $\left(\mathbf{x}_{1}, \mathbf{y}_{1}\right),\left(\mathbf{x}_{2}, \mathbf{y}_{2}\right), \ldots,\left(\mathbf{x}_{\mathbf{k}}, \mathbf{y}_{\mathbf{k}}\right)$, where $\mathbf{y}=\mathrm{f}(\mathbf{x})$

Example case 1: ${ }^{[1+1][15]}$

The research of Miami International Airport area ground-water contamination with three-dimensional coordinates and one contamination parameter has been completed. A back-propagation model with three layers has been constructed in the study, in which the three-dimensional coordinates were considered as the input nodes, one contamination parameter as the output node, and ten hidden nodes were designed. The 49 sample data sets were used to the neural network training with 0.15 learning rate and 0.075 momentum factor. The average error of the contamination estimations was less than $3 \%$. The map of the research area and estimation of the contamination distributions are presented in Figure 2.3 and 2.4.

Example case 2: ${ }^{[17]}$

A function with two input and one output $(f=\sin x+\sin y$ in $[0,2 \pi ; 0,2 \pi])$ has been studied. A back-propagation neural network model with four layers (one input layer with 2 nodes, two hidden layers with 15 nodes each and one output layer with 1 node) has been constructed. In this study, The 169 data sets were used for the neural network training with 0.6 learning rate and 0.9 momentum factor, and 144 data sets for its test. The average error of the estimation was less than $2.2 \%$. The actual (computational) and estimated results are presented in Figure 2.5 and 2.6. 


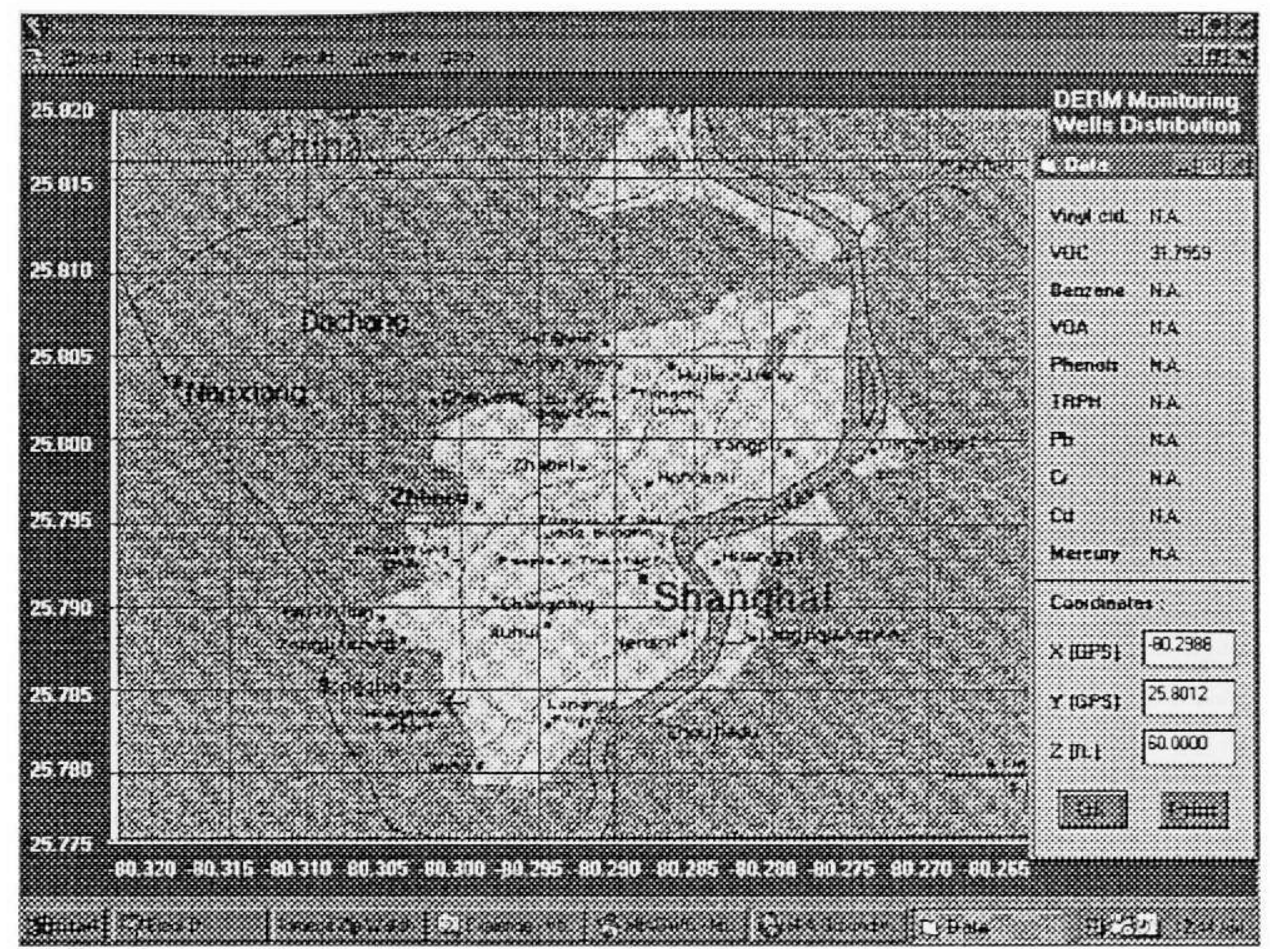

Figure 2.3 A research area map with the ground-water contamination data

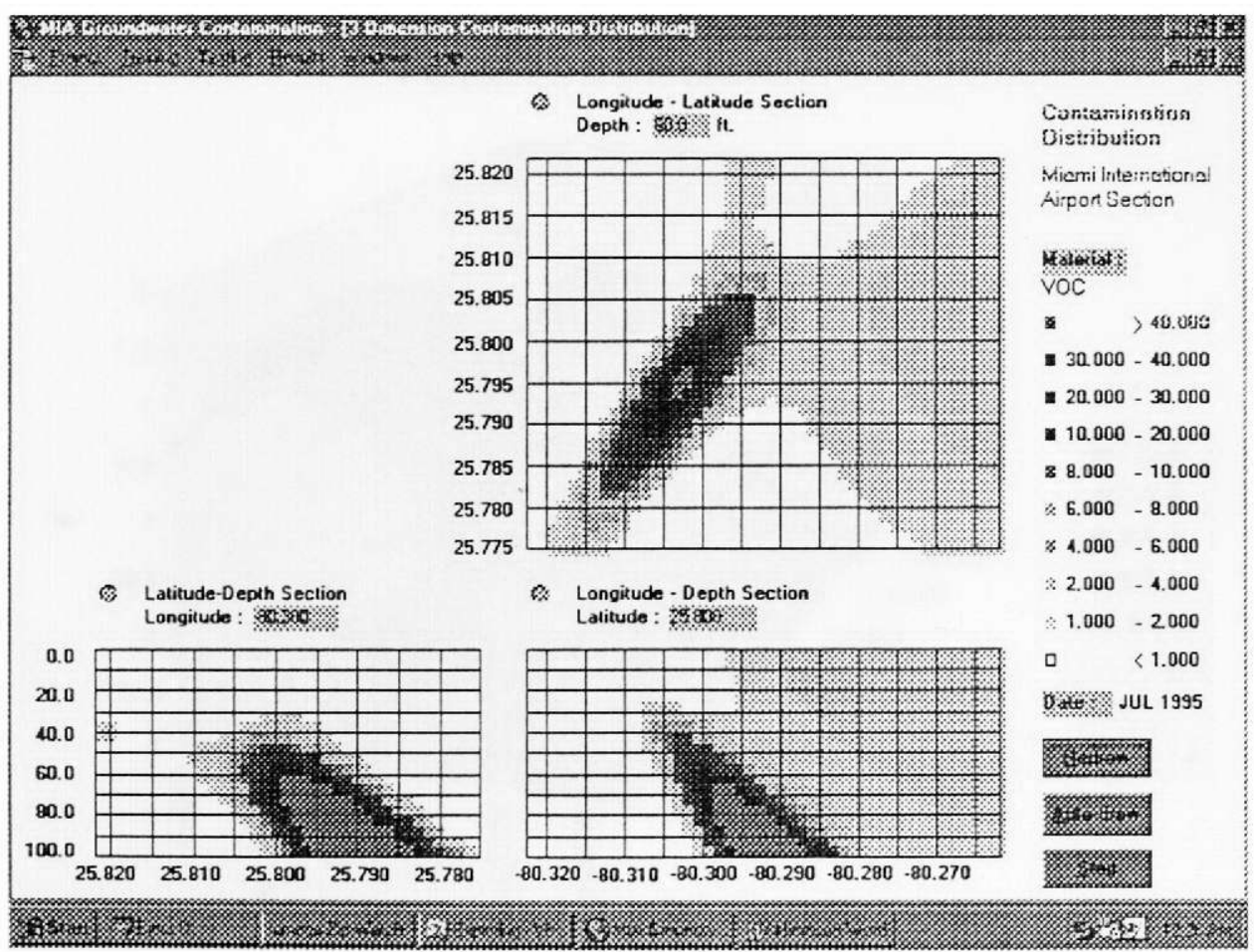

Figure 2.4 Estimation of the ground-water contamination distributions 


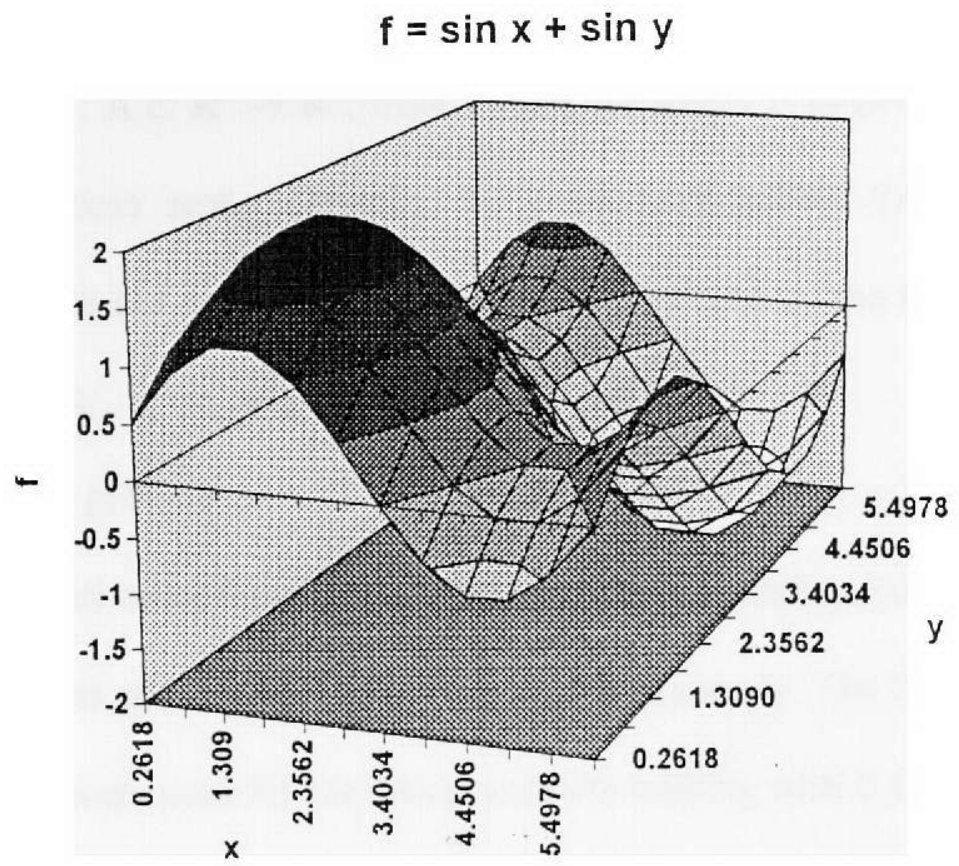

Figure 2.5 Computational results of a mapping study

\section{Function Estimation}

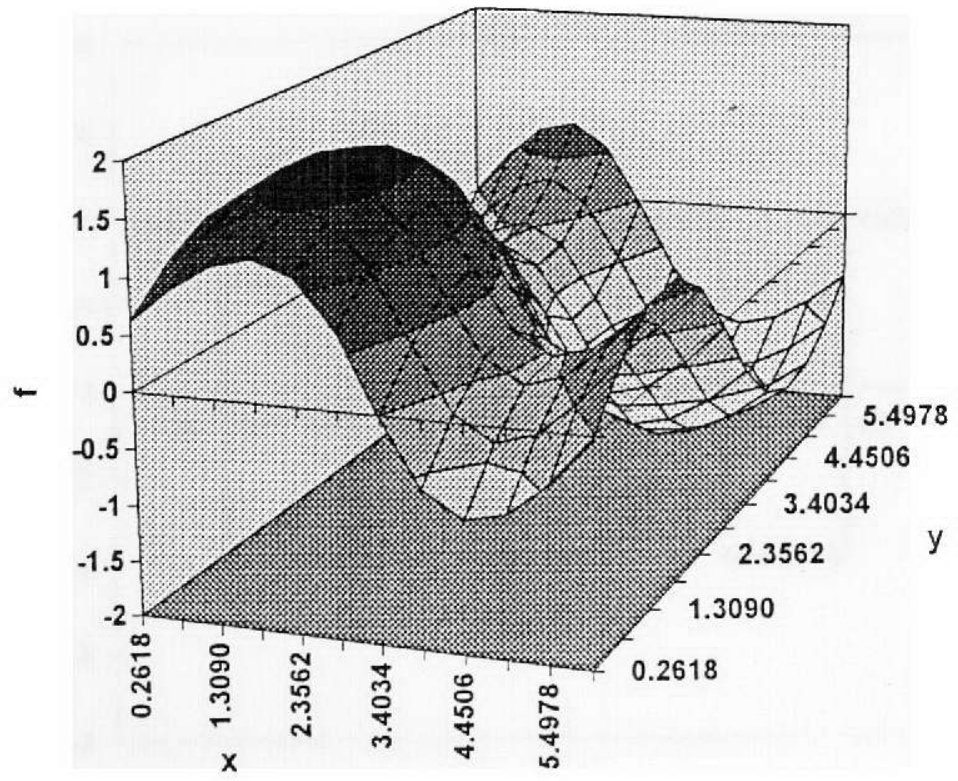

Figure 2.6 Estimated results of a mapping study 
Forecasting: The problem addressed by forecasting is the approximate implementation of a function $\mathrm{f}: \mathrm{A} \subset \mathbf{R}^{\mathrm{n}} \rightarrow \mathbf{R}^{\mathrm{m}}$, from a bounded subset $\mathrm{A}$ of ( $\left.\mathrm{n}-1\right)$-dimensional Euclidean space and a time series dimension to a bounded subset f[A] of (m-1)-dimensional Euclidean space and a time series dimension, by training on the time series example cases $\left(\mathbf{x}_{1}, \mathbf{y}_{1}\right),\left(\mathbf{x}_{2}, \mathbf{y}_{2}\right), \ldots,\left(\mathbf{x}_{\mathrm{k}}, \mathbf{y}_{\mathrm{k}}\right)$, where $\mathbf{y}=\mathrm{f}(\mathbf{x})$

\section{Example case 1: ${ }^{[15]}$}

A square wave function has been studied. A three-layer model with 5 input nodes, 16 hidden nodes and 1 output node was used in the study. The 216 data sets of the threeperiodic time were used for the neural network training with 0.15 learning rate and 0.075 momentum factor, and 72 data sets of the following period for its test. The average error of the estimation was less than $0.5 \%$. The results are presented in Figure 2.7.

\section{Square Wave Forecasting}

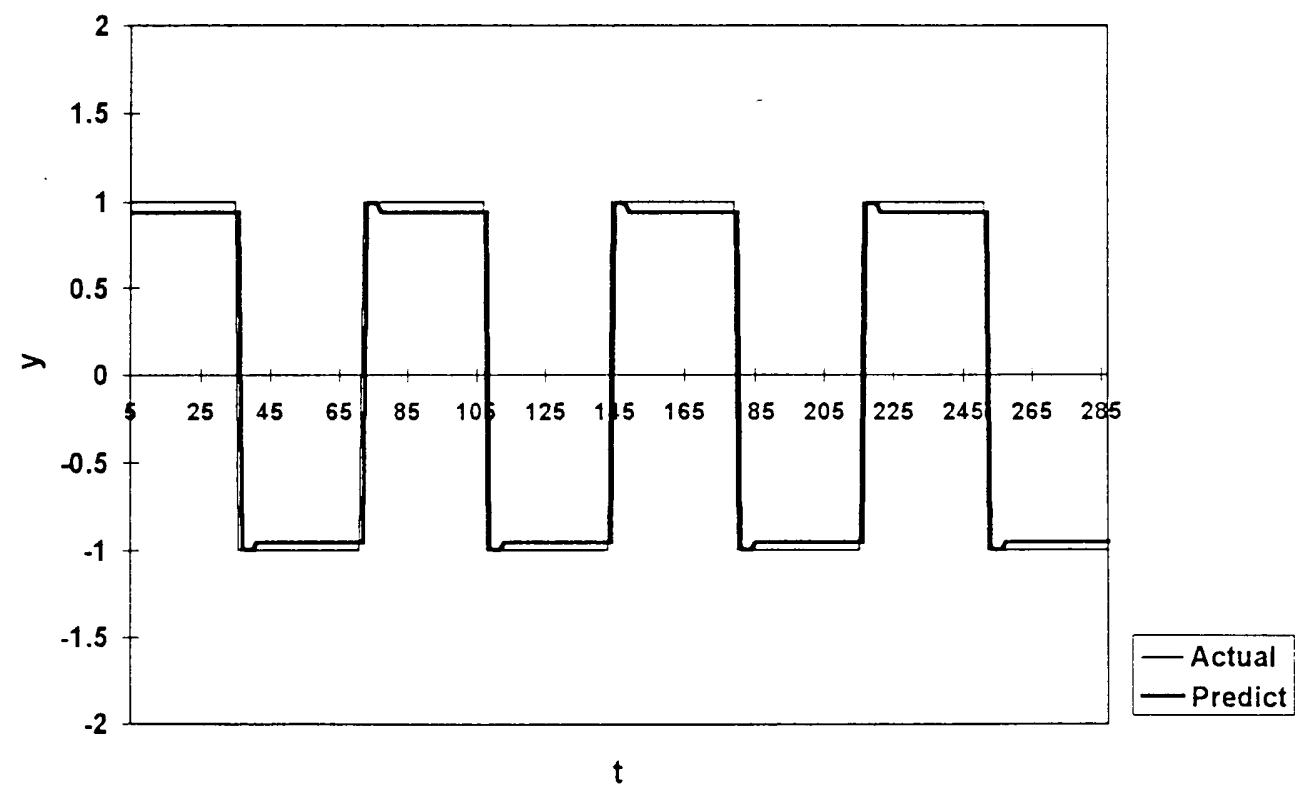

Figure 2.7 Results of square wave forecasting 


\section{Example case 2: ${ }^{[15]}$}

A exponential function with a exponential faded sine wave oscillation $\left(f=\left(1-e^{-t 2}\right)\right.$ $\left.+0.5 \mathrm{e}^{-t / 2} \sin (2 \pi t)\right)$ has been studied. A three-layer model with 3 input nodes, 10 hidden nodes and 1 output node was used in the study. The 72 data sets of the one-periodic time were used for the neural network training with 0.15 learning rate and 0.075 momentum factor, and 72 data sets of the following period for its test. The average error of the estimation was less than $2 \%$. The results are presented in Figure 2.8 .

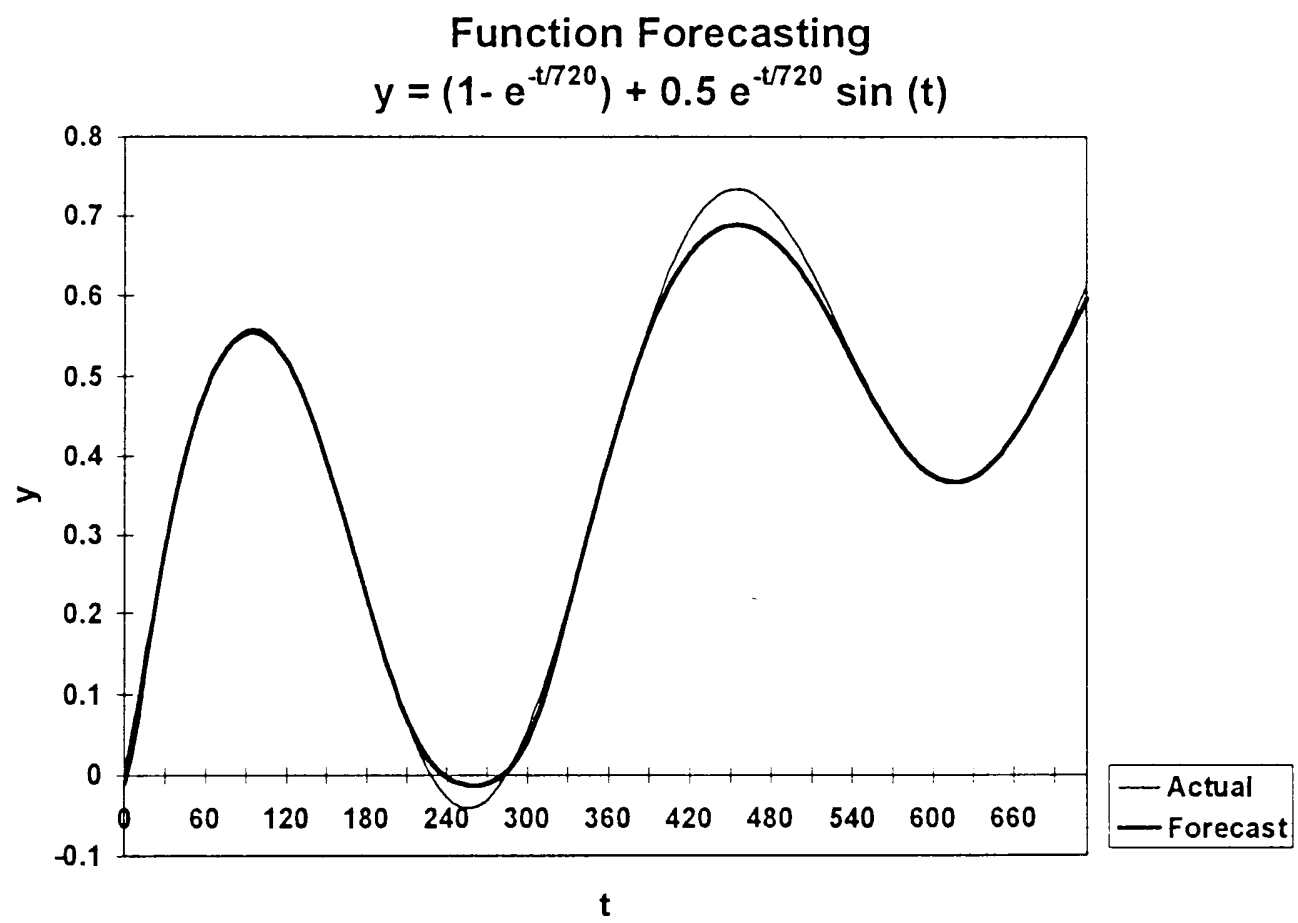

Figure 2.8 Results of function forecasting 


\subsection{Genetic Algorithms}

\section{Basic Theory of Genetic Algorithms}

Genetic algorithms are a class of dynamic computational models that mimic the natural selection and biological evolution to solve the real world problems. Based on biological theories, the deoxyribonucleic acid (DNA) molecule, a tiny corkscrew built from simple chemicals and included a large numbers of genes that define individual parts of the organism's blueprint, is the most fundamental piece carried life's mystery. The offspring inherit the characteristics from their parents through the genes. The survivals depended on natural selection (survival of the fittest) determine the characteristics of the next generation (the evolution of the species). ${ }^{[18]}$

The first application to solve problems by using the biological evolution was proposed in 1975 by John Holland. Based on the biomedical DNA theory, a binary number was designed as a individual chromosome to carry information of the data set, in which each bit presents a gene (see Figure 2.9). The individuals followed the biomedical DNA hereditary processes to mate each other and inherit a new generation through gene crossover and mutation. The natural selection as a rule determined the survivals of the next generation. The evolution would continue until one individual of the generation fitted the requested fitness level (see Figure 2.10).

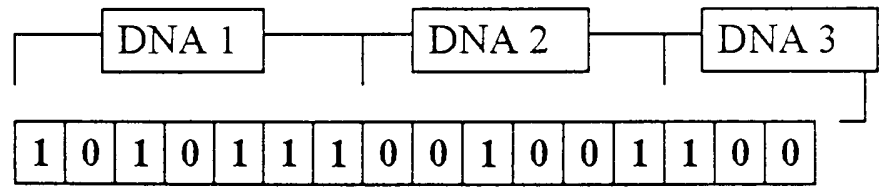

Figure 2.9 A data chromosome with 16 genes presented three DNA 


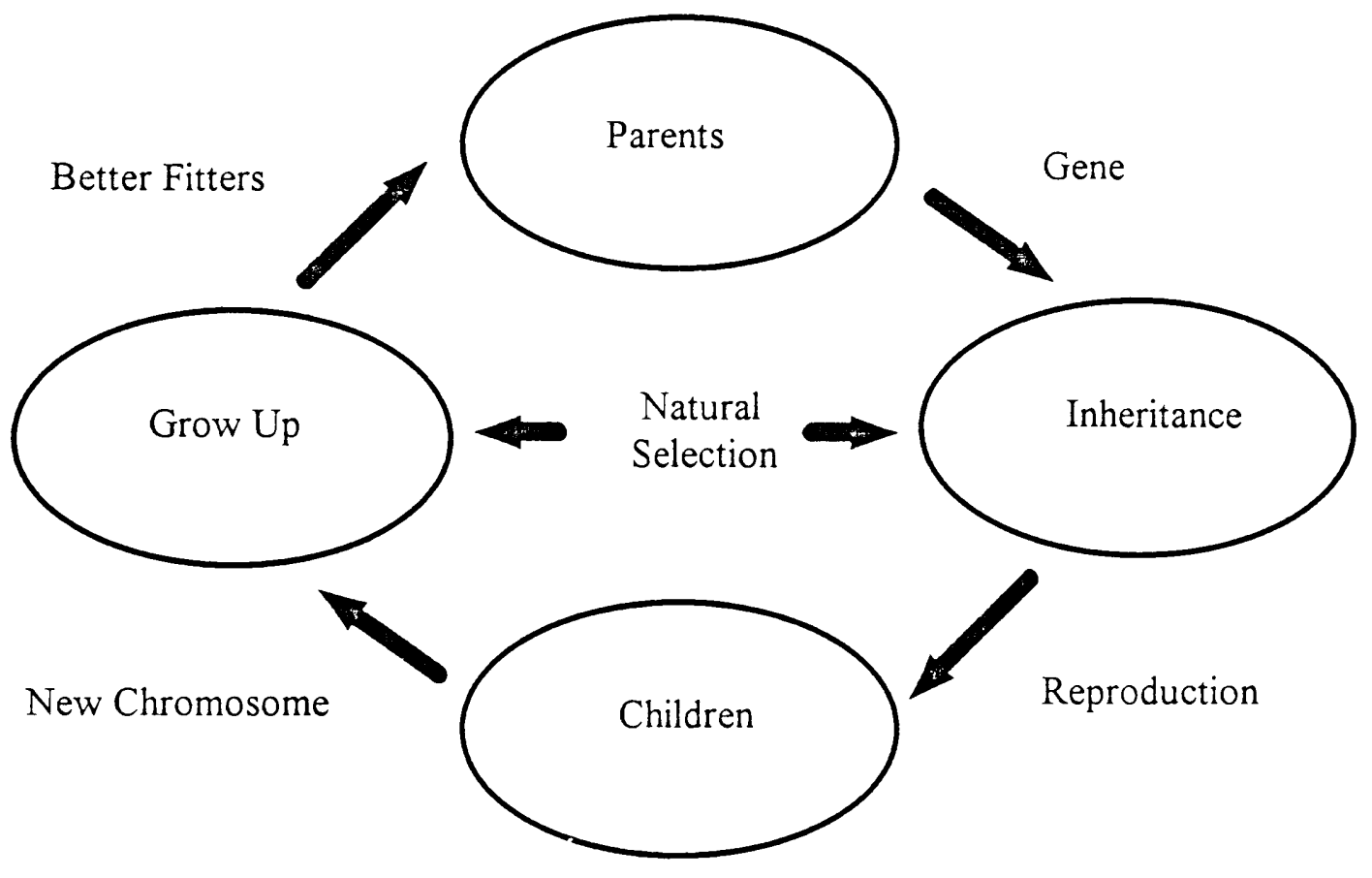

Figure 2.10 Biological evolution cycle of species

\section{Architecture of Genetic Algorithms}

In the view of the optimization, the genetic algorithms is a powerful tool to find the optimal solution of the problems with large data sets by using random search techniques. The natural selection rule is the key to search the optimal solution through the genetic evolution. The crossover operation keeps the better information from the last generation and the mutation operation helps to search the domain completely to avoid its being tripped in local maximum or minimum. The population size, mating pool size and the numbers of the children from each couple are decided by the problem study. The first generation can be randomly created and each following generation will be evolved by the following six steps: 
Step 1: Selection of the mating couples (parents).

The mating pools can be selected randomly or following some rules from the population pool. The mating couples can be picked randomly or following some rules from the mating pool. The procedure is presented in Figure 2.11.

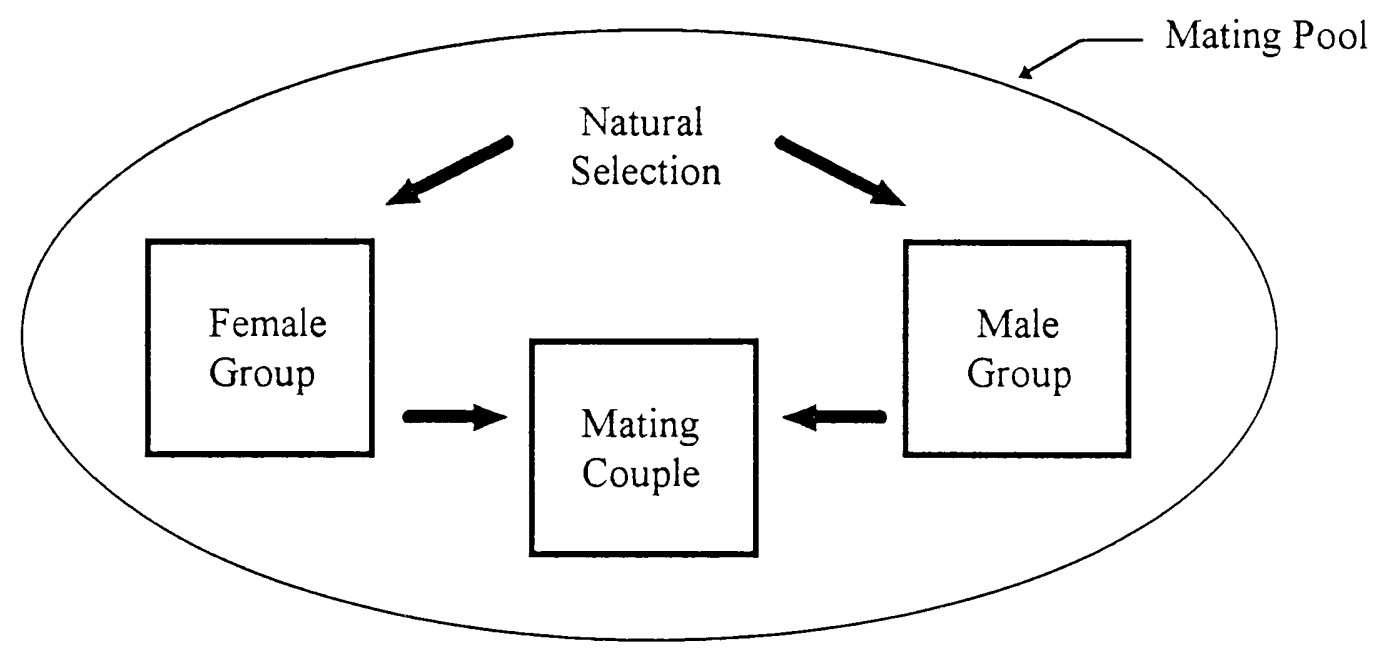

Figure 2.11 A mating procedure of genetic algorithms

Step 2: Selection of the hereditary chromosome of the next generation.

Three different methods are suggested to select the hereditary chromosomes.

1. The hereditary chromosome is always duplicated from the stronger one of the mating couple.

2. The hereditary chromosome is duplicated from the one of the mating couple by the turn.

3. The hereditary chromosome is randomly duplicated from the mating couple.

Step 3: Gene crossover.

Crossover is one of the genetic processes, in which the both parents' genes are 
combined to a new chromosome. There are two different processing theories to be applied to the operations, which are the fixed-point and uniform crossover.

Fixed-point crossover: Randomly pick a point in the chromosome, inherit all the genes from the one parent before that point and randomly inherit the genes from the both parents after the point (see Figure 2.12).

Crossover Point

Parent 1

\begin{tabular}{|l|l|l|l|l|l|l|l|l|l|l|l|l|l|l|l|}
\hline 1 & 0 & 1 & 0 & 1 & 1 & 1 & 0 & 0 & 1 & 0 & 0 & 1 & 1 & 0 & 0 \\
\hline
\end{tabular}

Parent 2

\begin{tabular}{|l|l|l|l|l|l|l|l|l|l|l|l|l|l|l|l|}
\hline 0 & 0 & 1 & 0 & 0 & 1 & 0 & 0 & 0 & 1 & 1 & 1 & 0 & 1 & 1 & 0 \\
\hline
\end{tabular}

Child

\begin{tabular}{|l|l|l|l|l|l|l|l|l|l|l|l|l|l|l|l|}
\hline 1 & 0 & 1 & 0 & 1 & 1 & 1 & 0 & 0 & 1 & 0 & 1 & 0 & 1 & 0 & 0 \\
\hline
\end{tabular}

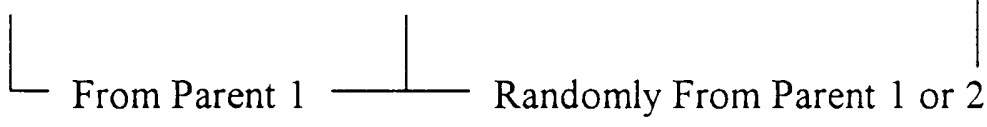

Figure 2.12 Fixed-point crossover genetic procedure

Uniform crossover: Randomly inherit the genes from the both parents in the whole chromosome (see Figure 2.13).

Parent 1

\begin{tabular}{|l|l|l|l|l|l|l|l|l|l|l|l|l|l|l|l|}
\hline 1 & 0 & 1 & 0 & 1 & 1 & 1 & 0 & 0 & 1 & 0 & 0 & 1 & 1 & 0 & 0 \\
\hline
\end{tabular}

Parent 2

\begin{tabular}{|l|l|l|l|l|l|l|l|l|l|l|l|l|l|l|l|}
\hline 0 & 0 & 1 & 0 & 0 & 1 & 0 & 0 & 0 & 1 & 1 & 1 & 0 & 1 & 1 & 0 \\
\hline
\end{tabular}

Child

\begin{tabular}{|l|l|l|l|l|l|l|l|l|l|l|l|l|l|l|l|}
\hline 0 & 0 & 1 & 0 & 1 & 1 & 0 & 0 & 0 & 1 & 0 & 1 & 0 & 1 & 0 & 0 \\
\hline
\end{tabular}

Randomly From Parent 1 or 2

Figure 2.13 Uniform crossover genetic procedure 
Step 4: Gene mutation.

Mutation is one of the genetic processes, in which the genes of the new chromosome are changed randomly according to the rapid evolution theories. There are two different processing theories to be applied to the operations, which are the jumping and creeping mutation.

Jumping mutation: Randomly change the genes of the chromosome in the certain jumping mutation probability lever (see Figure 2.14).

Before Jumping Mutation:

Child

\begin{tabular}{|l|l|l|l|l|l|l|l|l|l|l|l|l|l|l|l|}
\hline 0 & 0 & 1 & 0 & 1 & 1 & 0 & 0 & 0 & 1 & 0 & 1 & 0 & 1 & 0 & 0 \\
\hline
\end{tabular}

After Jumping Mutation: Randomly Mutated Genes

Child

\begin{tabular}{|l|l|l|l|l|l|l|l|l|l|l|l|l|l|l|l|}
\hline 0 & 0 & 1 & 0 & 1 & 1 & 0 & 0 & 1 & 1 & 0 & 0 & 0 & 1 & 0 & 0 \\
\hline
\end{tabular}

Figure 2.14 Jumping mutation genetic procedure

Creeping mutation: Randomly change a certain value of the chromosome data in the certain creeping mutation probability lever through a coding and decoding procedure.

Step 5: Creation of next generation (children).

There are two different theories to create new generations. One is that fittest individual of the one generation is always inherited to the next generations according to the elitism theory and the other is that fittest one is not necessary to be duplicated from one generation to the next one. 
Step 6: Evolution.

The children of this generation grow up and become the parents of the next one.

Repeat the six steps until the fitness is in the requested fitness level.

\section{Applications of Genetic Algorithms}

The Genetic algorithms can be applied to search a global optimal objective in high dimensional Euclidean space with a given function. A genetic algorithm program (GATool) was developed in 1998. It was successfully applied to monitor the cutting tool entry and exit angles of the micro-end-milling operations. ${ }^{[19]}$ In the research of micro-endmilling operations, it also has been used to estimate the tool breakage, wear, run-out and optimal working conditions (see Chapter V).

\section{Example case 1: ${ }^{[19]}$}

An identification program of the cutting tool entry and exit angles was developed by using genetic algorithms with an analytical cutting force model of micro-end-milling operations (see Chapter III). In all of the studied cases, the tool entry and exit cutting angles were estimated by the GATool program in less than 20 generations with less than $3 \%$ error. In 120 generations the error was reduced to less than $1 \%$. In the study, the population size was designed as five, mating pool size as two versus two and one child was created from each couple. 30 bit binary data was used for the individual coding. The uniform crossover with 0.5 probability, jumping mutation with 0.04 probability, creeping mutation with 0.02 probability and elitism were used in the genetic procedures. The results are presented in the Figure 2.15. 


\section{Geneic Evolution Procedure}

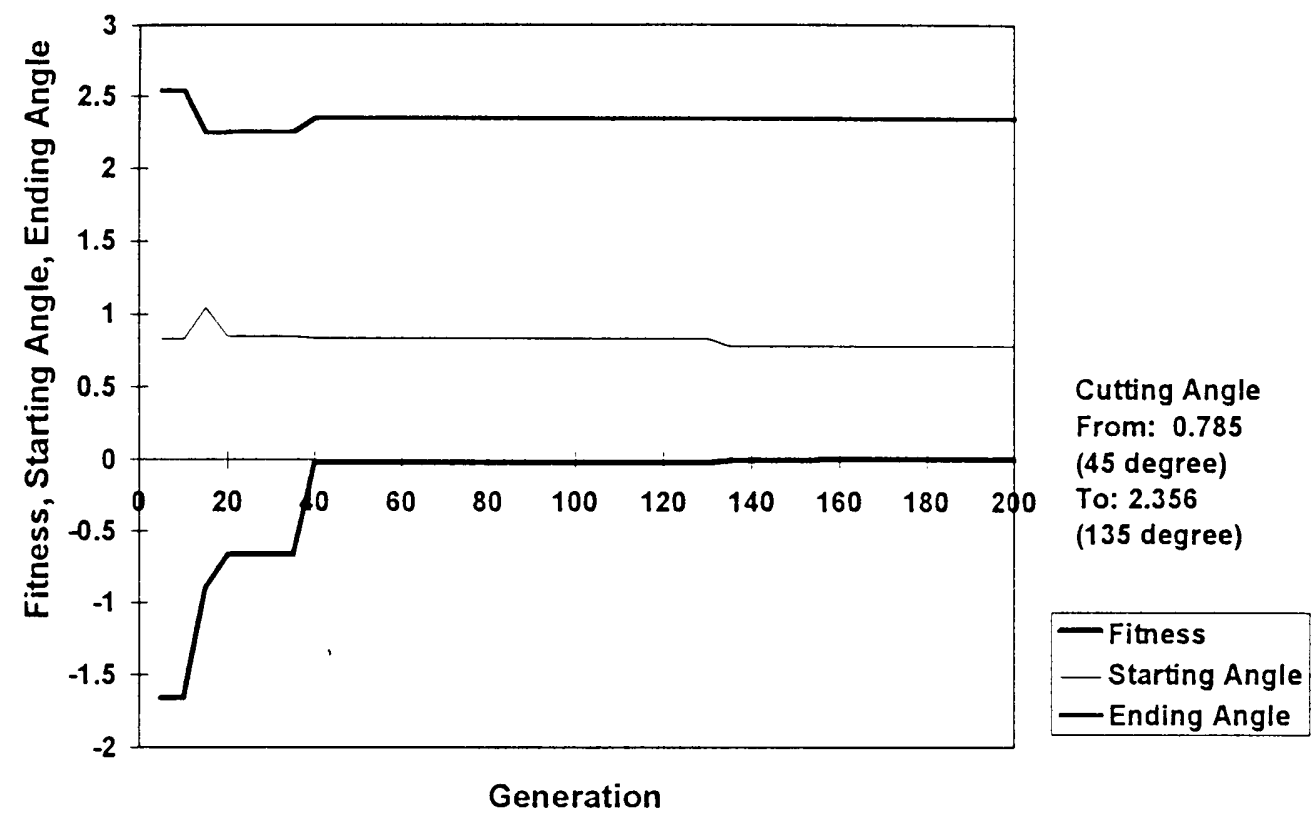

Figure 2.15 Genetic evolution procedure of the tool cutting angle monitoring

\section{Example case 2:}

A function with two parameters $\left(f=e^{(x+y)(2 \pi)}(\sin x+\sin y)\right.$ in $\left.[0,4 \pi ; 0,4 \pi]\right)$ has been studied. It has one global minimum, seven local minimum, eight local maximum and four stay points. The global minimum $(-69.615)$ of the function was found in less than 20 generations with less than $2.6 \%$ error and in 100 generation with less than $0.035 \%$ error. In the case, the population size was designed as five, mating pool size as two versus two and one child from each couple. 30 bit binary data was used for the individual coding. The uniform crossover with 0.5 probability, jumping mutation with 0.1 probability, creeping mutation with 0.05 probability and elitism were considered in the genetic procedures. The function and results are presented in Figure 2.16 and Figure 2.17. 


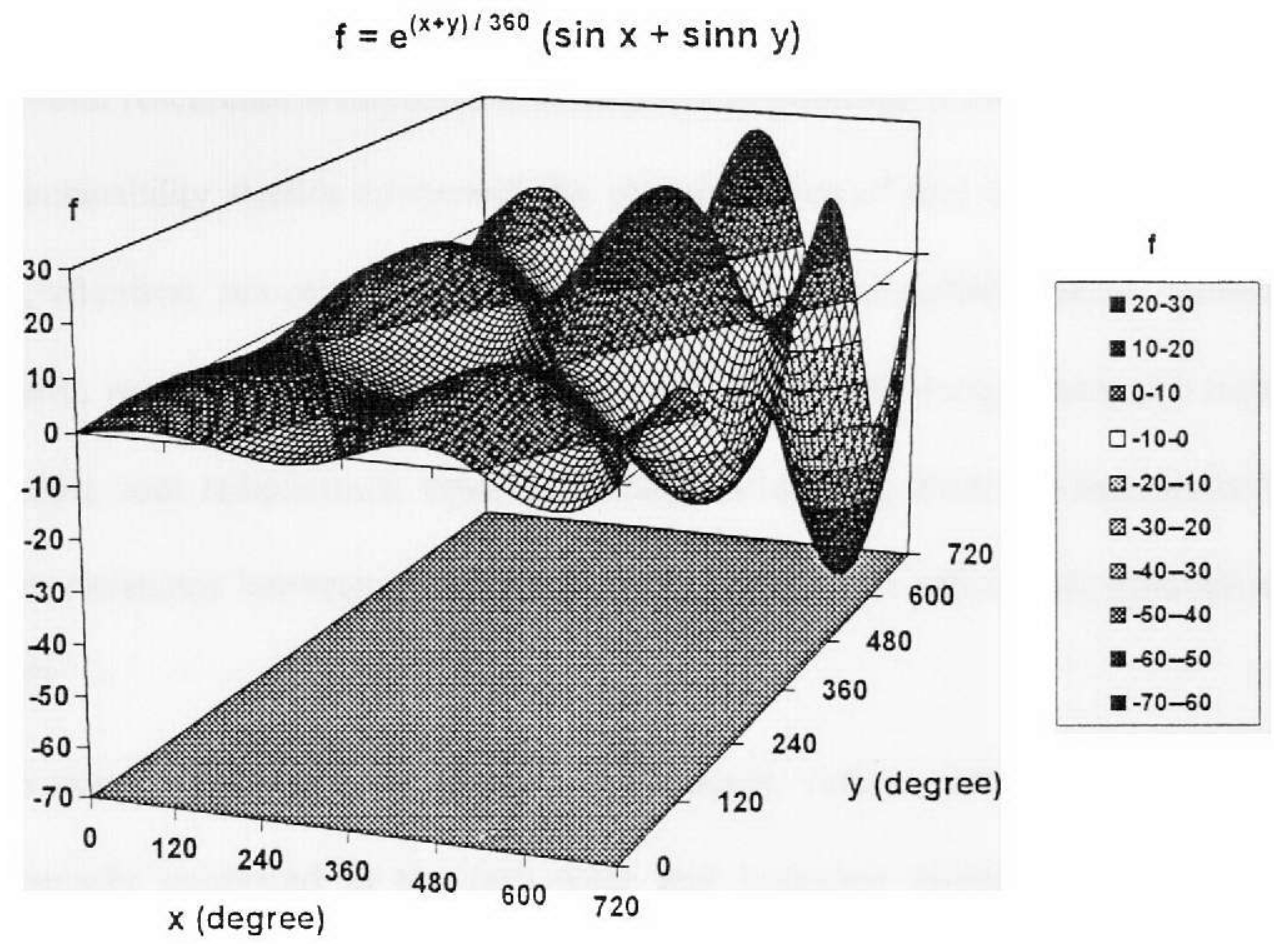

Figure 2.16 Function of an optimization case

\section{Genetic Evolution Procedure}

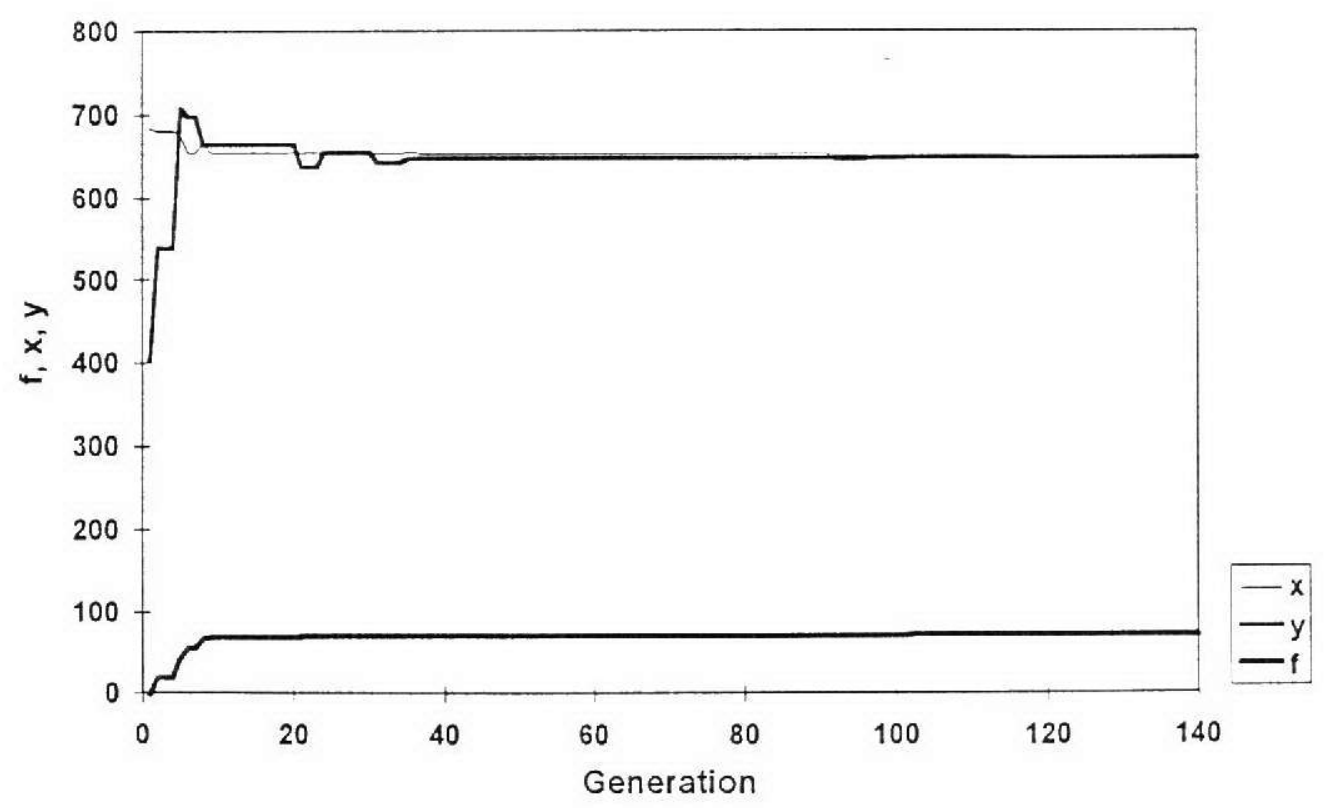

Figure 2.17 Genetic evolution procedure of the function optimization 


\subsection{Machinability and Monitoring of End Milling Operations}

Several researches studied machinability and monitoring of end milling operations.

Machinability studies concerned the characteristics of tool cutting force, torsion, deflection, vibration, run-out, wear, breakage and work-piece surface quality. To evaluate machinability, researchers measured tool cutting force, spindle torque, acoustic emission, tool vibration, tool temperature, optical scanning of tool tip, electrical measurements of the contact resistance between the tool and work-piece, radio active concentration at the chip. $^{[8][20-25]}$

To monitor tool wear and detect tool breakage, various methods were developed. It was specially interested in the tool wear and breakage monitoring, detection and control. Principle and Yoon detected the tool breakage by using displacement signals in 1991. ${ }^{[26]}$ Tansel et al. developed an on-line tool breakage detection system for end milling operations from acoustic emission and cutting force signals, and extend the tool life by reducing the feed rate in $1997 .{ }^{[27-31]}$ Liang and Dornfeld estimated tool wear from acoustic emission signals by using an on line time series model in $1989 .{ }^{[32]}$ Elbestawi et al. developed an on-line flank wear monitor system by monitoring cutting force signals. The harmonics of the cutting force spectrum were used for classification. ${ }^{[33]}$ Glass and Colbaugh estimated tool wear in metal cutting operations by directly using cutting force signals with neural networks in $1996^{[34]}$

To process experimental data, there are many different ways included geometry analyses, analysis methods, statistics, fuzzy mathematics, wavelet transform, neural networks and genetic algorithms. Statistical methods mainly used the average and 
deviation characteristics to avoid irregular information that was usually caused by noises in machining operations. The simple algorithms were applied to online tool breakage detection. ${ }^{[27-31][35][36]}$ Fuzzy mathematics was used to classify signals. It isolated the most important components of the objects and avoid unimportant details. It is a good way to analyze end milling operations because of the different characteristics of cutting force signals at different operating conditions. ${ }^{[37]}$ Wavelet transform, which is more efficient than Fourier transform when the signal is not a sine type, was used to compress any kinds of cyclical data to several simple characteristics ${ }^{[33][38][39]}$. Neural networks, a mimic human brain method, can be used for the data mapping, classification and forecasting. They was used to estimate tool cutting force and tool wear. ${ }^{[31][34][40-42]}$ Genetic algorithms, a mimic the natural selection and biological evolution method, were used for the functional optimization. ${ }^{[19]}$ 


\section{Chapter III}

\section{Analytical Cutting Force Model of Micro-End-Milling Operations}

The derivation of a new analytical cutting force model of end milling operations is presented in this chapter. The analytical model is developed for micro-end-milling operations. It also can be applied to conventional end milling operation. Ten parameters and two coefficients are considered in the model, which includes tool run-out and wear. They are three working condition variables (spindle speed, feed rate and depth of cut), two tool run-out variables (run-out and its angle), two cutting condition variables (tool cutting entry and exit angle) and three tool geometry variables (tool diameter, helix angle and the numbers of tool flutes). The material coefficient is related to the tool and workpiece materials. The tool wear coefficient is related to the tool life.

The developed analytical cutting force model has very good agreement with the experimental data of the micro-end-milling operations. The results are presented in Chapter VII.

\subsection{Cutting Force Model without Tool Run-out}

\section{Tool Cutting Edge and Its Tip Profile of End Milling Operations}

In end milling operations, the tip profile of the cutting edge of the tool (see Figure 3.1 ) is different from the one used by the third assumption of Tlusty's model (see Figure3.2). They are close only if the $\mathrm{f}_{\mathrm{t}} / \mathrm{r}$ is small enough to be neglected. 


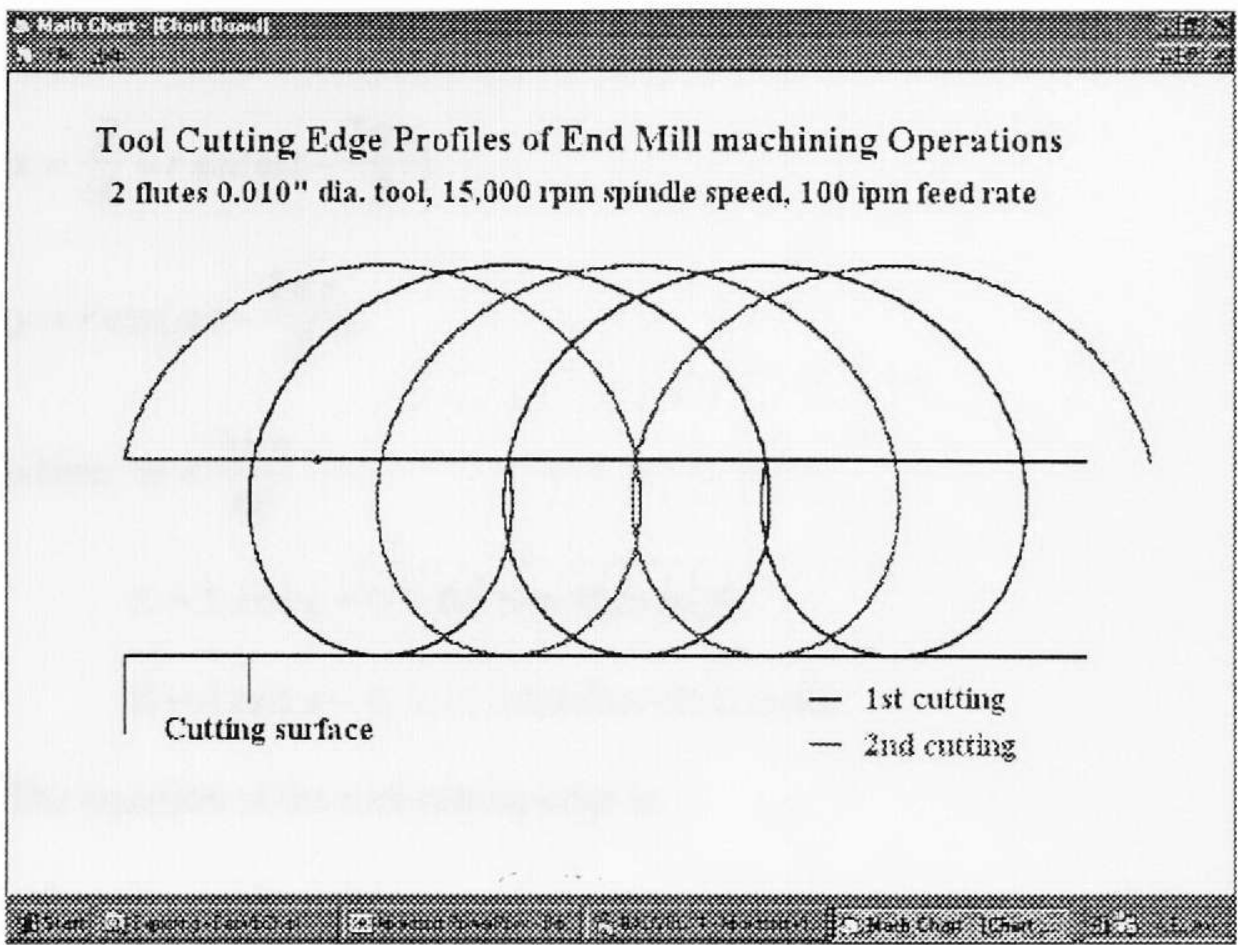

Figure 3.1 Tool cutting edge profiles of micro-end-milling operations

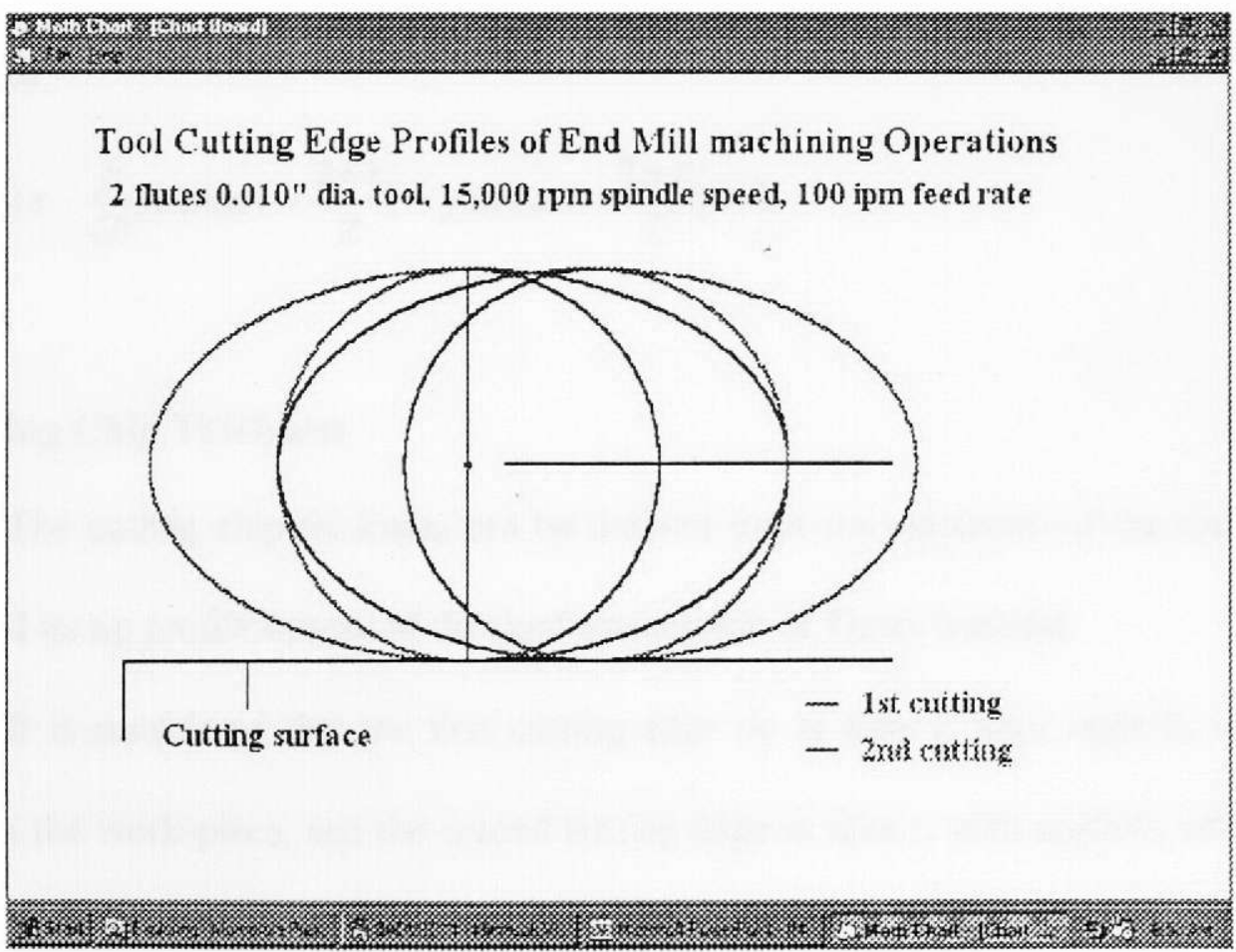

Figure 3.2 Tool cutting edge profiles of Tlusty's cutting force model 
The equations of the tool cutting edge tip profiles are:

$x=\frac{f t}{60}+r \sin \left(\omega t-\frac{2 \pi z}{Z}\right)$

$y=r \cos \left(\omega t-\frac{2 \pi z}{Z}\right)$

where: $\omega=\frac{2 \pi n}{60}$

$Z=2$ and $z=0,1$ for two-flute tools.

$Z=4$ and $z=0,1,2,3$ for four-flute tools.

The equation of the tool cutting edge is:

$\frac{x}{\frac{f t}{60}}-\frac{y}{\frac{f t}{60} \frac{1}{\tan \left(\omega t-\frac{2 \pi z}{Z}\right)}}=1$

or:

$\left(x-\frac{f t}{60}\right) \cos \left(\omega t-\frac{2 \pi z}{Z}\right)-y \sin \left(\omega t-\frac{2 \pi z}{Z}\right)=0$.

\section{Cutting Chip Thickness}

The cutting chip thickness can be derived from the equations of the tool cutting edge and its tip profile instead of the third assumption of Tlusty's model.

It is considered that the first cutting edge tip at time $t_{0}$ with angle $\theta_{0}$ reaches a point on the work-piece, and the second cutting edge at time $t_{1}$ with angle $\theta_{1}$ crosses that point. The following equations can be used: 


$$
\begin{aligned}
& \theta_{z}=\left(1+\frac{4 z}{Z}\right) \frac{\pi}{2}-\omega t_{z} \\
& \delta=\theta_{z+1}-\theta_{z} \\
& \omega t_{z+1}-\omega t_{z}=\frac{2 \pi}{Z}-\delta
\end{aligned}
$$

where: $z=0,1$ for two-flute tools.

$$
z=0,1,2,3 \text { for four-flute tools. }
$$

The equations3.1.1, 3.1.2 and 3.1.4 can also be rewritten as:

$$
\begin{aligned}
& x=\frac{f t_{0}}{60}+r \sin \left(\omega t_{0}-\frac{2 \pi z}{Z}\right) \\
& y=r \cos \left(\omega t_{0}-\frac{2 \pi z}{Z}\right) \\
& \left(x-\frac{f t_{1}}{60}\right) \cos \left(\omega t_{1}-\frac{2 \pi z}{Z}\right)-y \sin \left(\omega t_{1}-\frac{2 \pi z}{Z}\right)=0
\end{aligned}
$$

To solve the cross point, substitute $\mathrm{x}, \mathrm{y}$ from the equations3.1.8 and 3.1.9 to the equation 3.1.10.

$$
\begin{aligned}
& \left(\frac{f t_{0}}{60}-\frac{f t_{1}}{60}\right) \cos \left(\omega t_{1}-\frac{2 \pi(z+1)}{Z}\right)+r \sin \left(\omega t_{0}-\frac{2 \pi z}{Z}\right) \cos \left(\omega t_{1}-\frac{2 \pi(z+1)}{Z}\right) \\
& -r \cos \left(\omega t_{0}-\frac{2 \pi z}{Z}\right) \sin \left(\omega t_{1}-\frac{2 \pi(z+1)}{Z}\right)=0 \\
& \frac{f}{60 \omega}\left(\omega t_{0}-\omega t_{1}\right) \cos \left(\omega t_{1}-\frac{2 \pi(z+1)}{Z}\right)+r \sin \left(\omega t_{0}-\omega t_{1}+\frac{2 \pi}{Z}\right)=0
\end{aligned}
$$

Considering the equations3.1.5 to 3.1.7, the above equations can be simplified.

$$
\frac{f}{2 \pi n}\left(\frac{2 \pi}{Z}-\delta\right) \cos \left(\frac{\pi}{2}-\theta_{1}\right)=r \sin \delta
$$


Because $\theta_{0}$ and $\theta_{1}$ are very close, $\delta$ is a small angle. Let:

$\sin \delta=\delta$

and also let:

$\theta=\frac{\pi}{2}-\theta_{1}$

From the equation 3.1 .11 , the computing angle $\delta$ can be solved.

$$
\delta \approx \frac{f_{t} \frac{\cos \theta}{r}}{1+f_{t} \frac{Z \cos \theta}{2 \pi r}}
$$

where: $f_{t}=\frac{f}{n Z}$

The computing feed is:

$f_{c}=\frac{f}{60}\left(t_{1}-t_{0}\right)$

Considering the equations 3.1 .5 to 3.1 .7 , the equation 3.1 .13 can be rewritten as:

$f_{c}=\frac{f}{2 \pi n}\left(\frac{2 \pi}{Z}-\delta\right)$

The computing feed $f_{c}$ can be solved by substituting equation 3.1 .12 to it.

$f_{c} \approx \frac{f_{t}}{1+f_{t} \frac{Z \cos \theta}{2 \pi r}}$

Because of $f_{t} \frac{Z \cos \theta}{2 \pi r} \ll<1, \mathrm{f}_{\mathrm{c}}$ can be approximated as below:

$f_{c} \approx f_{t}\left(1-f_{t} \frac{Z \cos \theta}{2 \pi r}\right)$ 
In geometry view, computing feed can be obtained.

$$
\begin{aligned}
& \frac{\sin \delta}{f_{c}}=\frac{\sin \left(\pi-\theta_{1}\right)}{r} \\
& f_{c}=r \frac{\sin \delta}{\sin \left(\pi-\theta_{1}\right)}=r \frac{\sin \delta}{\cos \theta} \approx \frac{r \delta}{\cos \theta}=\frac{f_{t}}{1+f_{t} \frac{Z \cos \theta}{2 \pi r}}
\end{aligned}
$$

Also from geometry,

$r^{2}=H^{2}+f_{c}^{2}-2 H f_{c} \cos \left(\pi-\theta_{1}\right)$

$\mathrm{H}$ can be solved from the above equation,

$$
H=-f_{c} \sin \theta+\sqrt{r^{2}-\left(f_{c} \cos \theta\right)^{2}}
$$

The cutting chip thickness is:

$$
h=r-H=r+f_{c} \sin \theta-\sqrt{r^{2}-\left(f_{c} \cos \theta\right)^{2}}
$$

Because of $\frac{f_{c} \cos \theta}{r}<<1$, the cutting chip thickness can be approximated.

$$
h \approx f_{c} \sin \theta+\frac{1}{2 r}\left(f_{c} \cos \theta\right)^{2}
$$

Substituting the equation 3.1 .15 to 3.1 .19 , and also considering $f_{t}\left(\frac{f_{t}}{r}\right)^{2} \ll<f_{t}$ and $f_{t}\left(\frac{f_{t}}{r}\right)^{3} \ll f_{t}$, the cutting chip thickness becomes:

$$
h \approx f_{t} \sin \theta-\frac{Z}{2 \pi r} f_{t}^{2} \sin \theta \cos \theta+\frac{1}{2 r} f_{t}^{2} \cos ^{2} \theta
$$


If $\mathrm{f}_{\mathrm{t}} / \mathrm{r} \ll<1$, the formula becomes $\mathrm{h}=\mathrm{f}_{\mathrm{t}} \sin \theta$, this is the third assumption of Tlusty's model. It is proved that Tlusty's third assumption is satisfactory when $\mathrm{f}_{\mathrm{t}} / \mathrm{r}$ is small, which is encountered in most conventional end milling cases.

In the micro-end milling operations, $f_{t} / r$ is not small enough to be ignored. For example, if $n=15,000 \mathrm{rpm}, \mathrm{f}=100 \mathrm{ipm}, \mathrm{r}=0.01$ inch and $Z=2, f_{t} / r=1 / 3$, so that the second and third item of the formula 3.1.20 can't be neglected.

Let's look at the physical meaning of the formula 2.2.20.

The first term is major cutting chip thickness. It is considered in Tlusty's model for the cutting force calculation of conventional end milling operations.

The second term presents the difference between conventional milling and climbing milling. It is a negative valuable when $\theta$ is changed from 0 to 90 degree and a positive valuable from 90 to 180 degree. It is said that cutting chip thickness of climbing milling is thicker than that of conventional milling.

The third term is an additional cutting chip thickness. When $\theta=0$, the cutting chip thickness, which is equal to zero in Tlusty's model, is not equal to zero. It can be clearly known from Figure 3.1. There is a leading angle when $f_{t} / r$ value can't be neglected. It will be solved in following discussions.

The formula 2.2.20, which is derived from the general tool cutting profiles, do not have $z$ parameter. It is said that any cutting edge has the same cutting chip thickness in the same cutting angle. This is satisfactory in the case of conventional end milling operations without tool run-out. 


\section{Leading Angle}

In the formula 3.1 .20 , let $\mathrm{h}=0$. It becomes:

$$
\sin \lambda=-\frac{\frac{1}{2 r} f_{t} \cos ^{2} \lambda}{1-\frac{Z}{2 \pi r} f_{t} \cos \lambda}
$$

or:

$$
\sin \lambda \approx-\frac{f_{t}}{2 r} \cos ^{2} \lambda\left(1+f_{t} \frac{Z}{2 \pi r} \cos \lambda\right)
$$

Because $\theta$ closes to 0 or 180 degree, assume $\cos \theta=1$,

$$
\lambda \approx-\arcsin \left[\frac{f_{t}}{2 r}\left(1+f_{t} \frac{Z}{2 \pi r}\right)\right]
$$

$\lambda=|\lambda| \quad$ for conventional milling

$\lambda=\pi+|\lambda| \quad$ for climbing milling

For example, if $\mathrm{n}=15,000 \mathrm{rpm}, \mathrm{f}=100 \mathrm{ipm}, \mathrm{r}=0.01$ inch and $\mathrm{Z}=2, \lambda=-8.6^{\circ}$ in conventional milling or $\lambda=188.6^{\circ}$ in climbing milling.

If $\mathrm{f}_{\mathrm{t}} / \mathrm{r}$ is very small, this is $\mathrm{f}_{\mathrm{t}} / \mathrm{r} \approx 0, \lambda \approx 0^{\circ}$ in conventional milling or $\lambda \approx 180^{\circ}$ in climbing milling. That is the results of Tlusty's model.

\section{Cutting Force}

The cutting forces can be derived by using the formulas 2.1 .6 and 2.1.7, and cutting chip thickness formula 3.1.20 instead of the formula 2.1.3 (The third assumption of Tlusty's model). 
$d F_{x}=-2 F_{u}\left(\sin \theta-\frac{Z}{2 \pi r} f_{t} \sin \theta \cos \theta+\frac{1}{2 r} f_{t} \cos ^{2} \theta\right)(\cos \theta d \theta+p \sin \theta d \theta)$

$d F_{y}=2 F_{u}\left(\sin \theta-\frac{Z}{2 \pi r} f_{t} \sin \theta \cos \theta+\frac{1}{2 r} f_{t} \cos ^{2} \theta\right)(\sin \theta d \theta-p \cos \theta d \theta)$

where: $F_{u}=\frac{K_{w} K_{m} r f_{t}}{2 \tan \beta}$

Take the integration, the cutting force formulas can be derived.

$$
\begin{aligned}
& F_{x}=F_{u} {\left[\frac{1}{3} \frac{f_{t}}{r}\left(1+p \frac{Z}{\pi}\right)\left(\sin ^{3} \theta_{e}-\sin ^{3} \theta_{s}\right)+\frac{1}{3} \frac{f_{t}}{r}\left(p-\frac{Z}{\pi}\right)\left(\cos ^{3} \theta_{e}-\cos ^{3} \theta_{s}\right)-\left(\sin ^{2} \theta_{e}-\sin ^{2} \theta_{s}\right)\right.} \\
&\left.+\frac{1}{2} p\left(\sin 2 \theta_{e}-\sin 2 \theta_{s}\right)-\frac{f_{t}}{r}\left(\sin \theta_{e}-\sin \theta_{s}\right)-p\left(\theta_{e}-\theta_{s}\right)\right] \\
& F_{y}=F_{u}\left[\frac{1}{3} \frac{f_{t}}{r}\left(p-\frac{Z}{\pi}\right)\left(\sin ^{3} \theta_{e}-\sin ^{3} \theta_{s}\right)-\frac{1}{3} \frac{f_{t}}{r}\left(1+p \frac{Z}{\pi}\right)\left(\cos ^{3} \theta_{e}-\cos ^{3} \theta_{s}\right)-p\left(\sin ^{2} \theta_{e}-\sin ^{2} \theta_{s}\right)\right. \\
&\left.-\frac{1}{2}\left(\sin 2 \theta_{e}-\sin 2 \theta_{s}\right)-p \frac{f_{t}}{r}\left(\sin \theta_{e}-\sin \theta_{s}\right)+\left(\theta_{e}-\theta_{s}\right)\right]
\end{aligned}
$$

or:

$$
F_{x}=\left.F_{u}\left[C_{1} \frac{f_{t}}{r} \sin ^{3} \theta+C_{2} \frac{f_{t}}{r} \cos ^{3} \theta-\sin ^{2} \theta+\frac{1}{2} p \sin 2 \theta-\frac{f_{t}}{r} \sin \theta-p \theta\right]\right|_{\theta_{s}} ^{\theta_{e}}
$$

$$
\underline{F_{y}=\left.F_{u}\left[C_{2} \frac{f_{t}}{r} \sin ^{3} \theta-C_{1} \frac{f_{t}}{r} \cos ^{3} \theta-p \sin ^{2} \theta-\frac{1}{2} \sin 2 \theta-p \frac{f_{t}}{r} \sin \theta+\theta\right]\right|_{\theta_{s}} ^{\theta_{e}}}
$$

where : $C_{1}=\frac{1}{3}\left(1+p \frac{Z}{\pi}\right)$

$$
C_{2}=\frac{1}{3}\left(p-\frac{Z}{\pi}\right)
$$


Compared the cutting force model (formulas 3.1.28 and 3.1.29) to Tlusty's cutting force model (formulas 2.1.10 and 2.1.11), it is known that Tlusty's model is a special case of the model derived above when $f_{t} / r$ is small enough to be neglected.

Three different machining operation cases as same as ones mentioned in Tlusty's model are discussed.

Case 1: $\alpha \leq \varphi+\lambda$ and $\alpha+\varphi+\lambda \leq \psi$

For conventional milling:

section $1: \quad[-\lambda, \alpha-\lambda]$

$\theta_{\mathrm{s}}=-\lambda \quad \theta_{\mathrm{e}}=\theta$

section 2: $\quad[\alpha-\lambda, \varphi]$

$\theta_{\mathrm{s}}=\theta-\alpha \quad \theta_{\mathrm{e}}=\theta$

section 3: $\quad[\varphi, \varphi+\alpha]$

$\theta_{\mathrm{s}}=\theta-\alpha \quad \theta_{\mathrm{e}}=\varphi$

For climbing milling:

section 1: $\quad[\pi-\varphi, \pi-\varphi+\alpha] \quad \theta_{\mathrm{s}}=\pi-\varphi \quad \theta_{\mathrm{e}}=\theta$

section 2: $\quad[\pi-\varphi+\alpha, \pi+\lambda] \quad \theta_{\mathrm{s}}=\theta-\alpha \quad \theta_{\mathrm{e}}=\theta$

section 3: $\quad[\pi+\lambda, \pi+\lambda+\alpha] \quad \theta_{s}=\theta-\alpha \quad \theta_{e}=\pi+\lambda$

Case 2: $\alpha \geq \varphi+\lambda$ and $\alpha+\varphi+\lambda \leq \psi$

For conventional milling:

section $1: \quad[-\lambda, \varphi]$

$\theta_{\mathrm{s}}=-\lambda \quad \theta_{\mathrm{e}}=\theta$

section $2: \quad[\varphi, \alpha-\lambda]$

$\theta_{\mathrm{s}}=-\lambda$

$\theta_{\mathrm{e}}=\varphi$

section 3

$[\alpha-\lambda, \varphi+\alpha]$

$\theta_{s}=\theta-\alpha$

$\theta_{\mathrm{e}}=\varphi$ 
For climbing milling:

$$
\begin{array}{llll}
\text { section } 1: & {[\pi-\varphi, \pi+\lambda]} & \theta_{\mathrm{s}}=\pi-\varphi & \theta_{\mathrm{e}}=\theta \\
\text { section 2: } & {[\pi+\lambda, \pi-\varphi+\alpha]} & \theta_{\mathrm{s}}=\pi-\varphi & \theta_{\mathrm{e}}=\pi+\lambda \\
\text { section 3: } & {[\pi-\varphi+\alpha, \pi+\lambda+\alpha] \theta_{\mathrm{s}}=\theta-\alpha} & \theta_{\mathrm{e}}=\pi+\lambda
\end{array}
$$

Case 3: $\alpha+\varphi+\lambda \geq \psi$

Because of overlapping, the tool cutting force of the overlapped part is equal to the sum of the cutting forces of both cutting edges.

\subsection{Cutting Force Model with Tool Run-out}

\section{Tool Cutting Edge and Its Tip Profile of End Milling Operations}

The equations of the tool cutting edge tip profiles are:

$$
\begin{aligned}
& x=\frac{f t}{60}+r \sin \left(\omega t-\frac{2 \pi z}{Z}\right)+r_{o} \sin (\omega t+\gamma) \\
& y=r \cos \left(\omega t-\frac{2 \pi z}{Z}\right)+r_{o} \cos (\omega t+\gamma)
\end{aligned}
$$

where: $\omega=\frac{2 \pi n}{60}$

$$
\begin{aligned}
& Z=2 \text { and } z=0,1 \text { for two-flutes tool. } \\
& Z=4 \text { and } z=0,1,2,3 \text { for four-flute tool. }
\end{aligned}
$$

From geometry,

$$
\frac{\Delta f}{\sin \gamma}=\frac{r_{o}}{\sin \left(\frac{\pi}{2}+\omega t\right)}
$$


$\Delta f=r_{o} \frac{\sin \gamma}{\cos \omega t}$

$\Delta f^{2}=r_{o}^{2}+\Delta r^{2}-2 r_{o} \Delta r \cos \gamma$

$\Delta r=r_{o}(\cos \gamma-\sin \gamma \tan \omega t)$

The equation of the tool cutting edge is:

$\frac{x}{\frac{f t}{60}+r_{o} \frac{\sin \gamma}{\cos \omega t}}-\frac{y}{\left(\frac{f t}{60}+r_{o} \frac{\sin \gamma}{\cos \omega t}\right) \frac{1}{\tan \left(\omega t-\frac{2 \pi z}{Z}\right)}}=1$

or:

$\left[x-\left(\frac{f t}{60}+r_{o} \frac{\sin \gamma}{\cos \omega t}\right)\right] \cos \left(\omega t-\frac{2 \pi z}{Z}\right)-y \sin \left(\omega t-\frac{2 \pi z}{Z}\right)=0$

\section{Cutting Chip Thickness}

In the end milling with run-out case, each cutting edge has a different chip thickness. The two-flute tool is chosen in the following discussions.

(1) The chip thickness of the first cutter

The cutting chip thickness can be derived from the equations of the tool cutting edge and its tip profiles.

The equations 3.2.1, 3.2.2 and 3.2 .5 can be rewritten as:

$$
\begin{aligned}
& x=\frac{f t_{0}}{60}+r \sin \left(\omega t_{0}-\frac{2 \pi z}{Z}\right)+r_{o} \sin \left(\omega t_{0}+\gamma\right) \\
& y=r \cos \left(\omega t_{0}-\frac{2 \pi z}{Z}\right)+r_{o} \cos \left(\omega t_{0}+\gamma\right)
\end{aligned}
$$




$$
\left[x-\left(\frac{f t_{1}}{60}+r_{o} \frac{\sin \gamma}{\cos \omega t_{1}}\right)\right] \cos \left(\omega t_{1}-\frac{2 \pi z}{Z}\right)-y \sin \left(\omega t_{1}-\frac{2 \pi z}{Z}\right)=0
$$

As same as the discussion of the end milling without run-out case, to solve the cross point, substitute $\mathrm{x}, \mathrm{y}$ from the equations 3.2 .7 and 3.2 .8 to the equation 3.2.9.

$$
\begin{aligned}
& \left.\left[\left(\frac{f t_{0}}{60}-\frac{f t_{1}}{60}\right)-r_{o} \frac{\sin \gamma}{\cos \omega t_{1}}\right)\right] \cos \left(\omega t_{1}-\frac{2 \pi(z+1)}{Z}\right) \\
& +r \sin \left(\omega t_{0}-\frac{2 \pi z}{Z}\right) \cos \left(\omega t_{1}-\frac{2 \pi(z+1)}{Z}\right)-r \cos \left(\omega t_{0}-\frac{2 \pi z}{Z}\right) \sin \left(\omega t_{1}-\frac{2 \pi(z+1)}{Z}\right) \\
& +r_{o} \sin \left(\omega t_{0}+\gamma\right) \cos \left(\omega t_{1}-\frac{2 \pi(z+1)}{Z}\right)-r_{o} \cos \left(\omega t_{0}+\gamma\right) \sin \left(\omega t_{1}-\frac{2 \pi(z+1)}{Z}\right)=0 \\
& {\left[\frac{f}{60 \omega}\left(\omega t_{0}-\omega t_{1}\right)-r_{o} \frac{\sin \gamma}{\cos \omega t_{1}}\right] \cos \left(\omega t_{1}-\frac{2 \pi(z+1)}{Z}\right)} \\
& +r \sin \left(\omega t_{0}-\omega t_{1}+\frac{2 \pi}{Z}\right)+r_{o} \sin \left(\omega t_{0}-\omega t_{1}+\gamma+\frac{2 \pi(z+1)}{Z}\right)=0
\end{aligned}
$$

Considering the equations 3.1 .5 to 3.1 .7 , the above equation can be simplified.

$$
\begin{aligned}
& \left\{\frac{f}{2 \pi n}\left(\frac{2 \pi}{Z}-\delta\right)+r_{o} \frac{\sin \gamma}{\cos \left[\frac{\pi}{2}-\theta_{1}+\frac{2 \pi(z+1)}{Z}\right]}\right\} \cos \left(\frac{\pi}{2}-\theta_{1}\right) \\
& =r \sin \delta+r_{o} \sin \left(\delta+\gamma+\frac{2 \pi z}{Z}\right)
\end{aligned}
$$

Because $\theta_{0}$ and $\theta_{1}$ are very close, $\delta$ is a small angle. Let:

$\sin \delta=\delta$

and also let:

$$
\theta=\frac{\pi}{2}-\theta_{1}
$$


From the equation 3.2.10, the computing angle $\delta$ can be solved.

$$
\approx \frac{f_{t} \frac{\cos \theta}{r}+\frac{r_{o}}{r} \frac{\sin \gamma \cos \theta}{\cos \left[\theta+\frac{2 \pi(z+1)}{Z}\right]}-\frac{r_{o}}{r} \sin \left(\gamma+\frac{2 \pi z}{Z}\right)}{1+f_{t} \frac{Z \cos \theta}{2 \pi r}+\frac{r_{o}}{r} \cos \left(\gamma+\frac{2 \pi z}{Z}\right)}
$$

where: $f_{t}=\frac{f}{n Z}$

Let's consider the two-flute end mill case. The equation 3.2.11 can be simplified.

$$
\delta \approx \frac{f_{t} \frac{\cos \theta}{r}-(-1)=2 \frac{r_{o}}{r} \sin \gamma}{1+f_{t} \frac{\cos \theta}{\pi r}+(-1)^{2} \frac{r_{o}}{r} \cos \gamma}
$$

where: $z=0,1$

Considering $\frac{f_{t}}{r} \frac{r_{o}}{r} \ll<\frac{f_{t}}{r},\left(\frac{r_{o}}{r}\right)^{2} \ll \frac{r_{o}}{r}, \frac{f_{t}}{\pi r} \ll 1$ and $\frac{r_{o}}{r} \ll<$, the equation 3.2 .12 can be rewritten as:

$$
\delta \approx f_{t} \frac{\cos \theta}{r}-(-1)^{2} 2 \frac{r_{o}}{r} \sin \gamma-\frac{1}{\pi}\left(f_{t} \frac{\cos \theta}{r}\right)^{2}
$$

The computing feed is:

$$
f_{c}=\frac{f}{60}\left(t_{1}-t_{0}\right)+r_{o} \sin \gamma\left(\frac{1}{\cos \omega t_{1}}-\frac{1}{\cos \omega t_{0}}\right)
$$

Considering the equations 3.1 .5 to 3.1 .7 and $\theta_{0} \approx \theta_{1}$ because of $\delta$ small, the equation 3.2.14 can be rewritten as:

$$
f_{c}=\frac{f}{2 \pi n}(\pi-\delta)-(-1)^{2} 2 r_{o} \frac{\sin \gamma}{\cos \theta}
$$


Substituting the equation 3.2.12 to it, the computing feed $f_{c}$ can be solved.

$$
f_{c} \approx f_{t}\left[1+(-1)^{z} \frac{2 r_{o}}{\pi r} \sin \gamma\right]-f_{t}^{2} \frac{\cos \theta}{\pi r}-(-1)^{z} 2 r_{o} \frac{\sin \gamma}{\cos \theta}
$$

Because of $\theta_{0} \approx \theta_{1}$, assumed $\Delta r_{0} \approx \Delta r_{1}=\Delta r$. From geometry,

$$
\left[r+(-1)^{2} \Delta r\right]^{2}=H^{2}+f_{c}^{2}-2 H f_{c} \cos \left(\pi-\theta_{1}\right)
$$

$\mathrm{H}$ can be solved from above equation:

$$
H=-f_{c} \sin \theta+\sqrt{\left[r+(-1)^{2} \Delta r\right]^{2}-\left(f_{c} \cos \theta\right)^{2}}
$$

The cutting chip thickness is:

$$
\begin{aligned}
h & =\left[r-(-1)^{2} \Delta r\right]-H \\
& =\left[r-(-1)^{2} \Delta r\right]+f_{c} \sin \theta-\sqrt{\left[r+(-1)^{2} \Delta r\right]^{2}-\left(f_{c} \cos \theta\right)^{2}}
\end{aligned}
$$

Because of $\frac{f_{c} \cos \theta}{r} \ll<1$, the cutting chip thickness can be approximated.

$$
h \approx f_{c} \sin \theta+\frac{1}{2\left[r+(-1)^{2} \Delta r\right]}\left(f_{c} \cos \theta\right)^{2}-(-1)^{2} 2 \Delta r
$$

Substituting the equation 2.3 .15 to 2.3 .19 , and also considering $f_{t}\left(\frac{f_{t}}{r}\right)^{2} \ll<f_{t}$ and $f_{t}\left(\frac{f_{t}}{r}\right)^{3} \ll f_{t}$, the cutting chip thickness becomes:

$$
\left.h \approx f_{t}\left[1+(-1)^{z} \frac{2 r_{o}}{\pi r} \sin \gamma\right)\right] \sin \theta-\frac{1}{\pi r} f_{t}^{2} \sin \theta \cos \theta+\frac{1}{2 r} f_{t}^{2} \cos ^{2} \theta-(-1)^{z} 2 r_{o} \cos \gamma
$$


If $f_{t} / r<1$ and $r_{0}=0$, the formula becomes $h=f_{t} \sin \theta$, this is the third assumption of Tlusty's model. It is said that Tlusty's model is a special case of end milling without run-out.

Comparing the formula 3.2.20 to the formula 3.1.20, The tool run-out is expressed in the first and forth items of the formula 3.2.20.

The forth term of the formula 3.2 .20 is a major run-out factor. It reaches a maximum value when the tool run-out is parallel to the tool cutting edge ( $\gamma=0$ degree), and turns to a minimum value when the tool run-out is perpendicular to the tool cutting edge $(\gamma=90$ degree $)$.

The second part of the first term the formula 3.2.20 is an additional run-out factor. When $\gamma=90$ degree, the tool run-out turns into a minimum level and almost disappear. It can be neglected in most conventional end milling operations because of $r_{0} / r<<1$,

For two-flute end-mills, if $2 r_{0} \cos \gamma$ is larger than $\mathrm{f}_{\mathrm{t}}$, only one cutting edge works in the machining operations.

The formula 3.2.20, which is derived from the general tool cutting profiles, do have $\mathrm{z}$ parameter. It is said that different cutting edges have the deferent cutting chip thickness. This is a true in the case of end milling with run-out.

Let $z=0$, the chip thickness of the first cutter is:

$$
\left.h \approx f_{t}\left[1+\frac{2 r_{o}}{\pi r} \sin \gamma\right)\right] \sin \theta-\frac{1}{\pi r} f_{t}^{2} \sin \theta \cos \theta+\frac{1}{2 r} f_{t}^{2} \cos ^{2} \theta-2 r_{o} \cos \gamma
$$


(2) The chip thickness of the second cutter

The second cutter chip thickness calculation can be considered into two sections. In the first section, the second cutting path of the second cutter cuts on its first cutting path. In the second section, the second cutting path of the second cutter cuts on the first cutting path of the first cutter path.

A. In the first section of the cutting operations

The equations $3.2 .1,3.2 .2$ and 3.2 .5 can be rewritten as:

$$
\begin{aligned}
& x=\frac{f t_{0}}{60}+r \sin \left(\omega t_{0}-\frac{2 \pi z}{Z}\right)+r_{o} \sin \left(\omega t_{0}+\gamma\right) \\
& y=r \cos \left(\omega t_{0}-\frac{2 \pi z}{Z}\right)+r_{o} \cos \left(\omega t_{0}+\gamma\right) \\
& {\left[x-\left(\frac{f t_{1}}{60}+r_{o} \frac{\sin \gamma}{\cos \omega t_{2}}\right)\right] \cos \left(\omega t_{2}-\frac{2 \pi z}{Z}\right)-y \sin \left(\omega t_{2}-\frac{2 \pi z}{Z}\right)=0}
\end{aligned}
$$

As same as the above discussion, to solve the cross point, substitute $\mathrm{x}$, $\mathrm{y}$ from the equations 3.2 .22 and 3.2 .23 to the equation 3.2.24.

$$
\begin{aligned}
& \left.\left[\left(\frac{f t_{0}}{60}-\frac{f t_{1}}{60}\right)-r_{o} \frac{\sin \gamma}{\cos \omega t_{2}}\right)\right] \cos \left(\omega t_{2}-\frac{2 \pi(z+2)}{Z}\right) \\
& +r \sin \left(\omega t_{0}-\frac{2 \pi z}{Z}\right) \cos \left(\omega t_{2}-\frac{2 \pi(z+2)}{Z}\right)-r \cos \left(\omega t_{0}-\frac{2 \pi z}{Z}\right) \sin \left(\omega t_{2}-\frac{2 \pi(z+2)}{Z}\right) \\
& +r_{o} \sin \left(\omega t_{0}+\gamma\right) \cos \left(\omega t_{2}-\frac{2 \pi(z+2)}{Z}\right)-r_{o} \cos \left(\omega t_{0}+\gamma\right) \sin \left(\omega t_{2}-\frac{2 \pi(z+2)}{Z}\right)=0 \\
& \quad\left[\frac{f}{60 \omega}\left(\omega t_{0}-\omega t_{2}\right)-r_{o} \frac{\sin \gamma}{\cos \omega t_{2}}\right] \cos \left(\omega t_{2}-\frac{2 \pi(z+2)}{Z}\right) \\
& +r \sin \left(\omega t_{0}-\omega t_{2}+\frac{4 \pi}{Z}\right)+r_{o} \sin \left(\omega t_{0}-\omega t_{2}+\gamma+\frac{2 \pi(z+2)}{Z}\right)=0
\end{aligned}
$$


Considering the equations 3.1 .5 to 3.1 .7 , the above equation can be simplified.

$$
\begin{aligned}
& \left\{\frac{f}{2 \pi n}\left(\frac{4 \pi}{Z}-\delta\right)+r_{o} \frac{\sin \gamma}{\cos \left[\frac{\pi}{2}-\theta_{2}+\frac{2 \pi(z+2)}{Z}\right]}\right\} \cos \left(\frac{\pi}{2}-\theta_{2}\right) \\
& =r \sin \delta+r_{o} \sin \left(\delta+\gamma+\frac{2 \pi z}{Z}\right)
\end{aligned}
$$

Because $\theta_{0}$ and $\theta_{1}$ are very close, $\delta$ is a small angle. Let:

$\sin \delta=\delta$

and also let:

$$
\theta=\frac{\pi}{2}-\theta_{2}
$$

From the equation 2.3.25, the computing angle $\delta$ can be solved.

$$
\delta \approx \frac{\left.2 f_{t} \frac{\cos \theta}{r}\right)}{1+f_{t} \frac{Z \cos \theta}{2 \pi r}+\frac{r_{o}}{r} \cos \left(\gamma+\frac{2 \pi z}{Z}\right)}
$$

where: $f_{t}=\frac{f}{n Z}$

Let's consider the two-flute end-mill case. The equation 3.2.26 can be simplified.

$$
\delta \approx \frac{2 f_{t} \frac{\cos \theta}{r}}{1+f_{t} \frac{\cos \theta}{\pi r}+\frac{r_{o}}{r} \cos \gamma}
$$

Considering $\frac{f_{t}}{r} \frac{r_{o}}{r}<<\frac{f_{t}}{r},\left(\frac{r_{o}}{r}\right)^{2}<<\frac{r_{o}}{r}, \frac{f_{t}}{\pi r}<<1$ and $\frac{r_{o}}{r}<<1$, the equation 3.2 .27 can be rewritten as: 
$\delta \approx 2 f_{t} \frac{\cos \theta}{r}-\frac{2}{\pi}\left(f_{t} \frac{\cos \theta}{r}\right)^{2}$

The computing feed is:

$$
f_{c}=\frac{f}{60}\left(t_{2}-t_{0}\right)+r_{o} \sin \gamma\left(\frac{1}{\cos \omega t_{2}}-\frac{1}{\cos \omega t_{0}}\right)
$$

Considering the equations 3.1 .5 to 3.1 .7 ; and $\theta_{0} \approx \theta_{2}$ because of $\delta$ small, the equation 3.1 .14 can be rewritten as:

$$
f_{c}=\frac{f}{2 \pi n}(2 \pi-\delta)
$$

Substituting equation 3.2.27 to it, the computing feed $f_{c}$ can be solved.

$$
f_{c} \approx 2 f_{t}-2 f_{t}^{2} \frac{\cos \theta}{\pi r}
$$

Substituting the equation 2.3.30 to 2.2.19, and also considering $f_{t}\left(\frac{f_{t}}{r}\right)^{2} \ll<f_{t}$ and $f_{t}\left(\frac{f_{t}}{r}\right)^{3} \ll<f_{t}$, the cutting chip thickness becomes:

$$
h \approx 2 f_{t} \sin \theta-\frac{2}{\pi r} f_{t}^{2} \sin \theta \cos \theta+\frac{4}{2 r} f_{t}^{2} \cos ^{2} \theta
$$

B. In the second section of the cutting operations

In the formula 3.2.20, let $z=1$. The chip thickness of the second cutter becomes:

$$
\left.h \approx f_{t}\left[1-\frac{2 r_{o}}{\pi r} \sin \gamma\right)\right] \sin \theta-\frac{1}{\pi r} f_{i}^{2} \sin \theta \cos \theta+\frac{1}{2 r} f_{t}^{2} \cos ^{2} \theta+2 r_{o} \cos \gamma
$$




\section{Integrating Angle}

(1) The integrating angle of the first cutter

In the formula 3.2 .21 , let $h=0$.

$$
\sin \lambda_{1}=\frac{2 r_{o} \cos \gamma-\frac{1}{2 r} f_{t}^{2} \cos ^{2} \lambda_{1}}{f_{t}\left(1+\frac{2 r_{o}}{\pi r} \sin \gamma\right)-\frac{1}{\pi r} f_{t}^{2} \cos \lambda_{1}}
$$

If $\mathrm{f}_{\mathrm{t}} / \mathrm{r}<<1$ and $\mathrm{r}_{\mathrm{o}} / \mathrm{r}<<1$, it becomes:

$$
\lambda_{1}=\arcsin \left(\frac{2 r_{o}}{f_{t}} \cos \gamma\right)
$$

(2) The integrating angle of the second cutter

A. The first section

In the formula 2.3.31, let $\mathrm{h}=0$,

$$
\sin \lambda_{21}=-\frac{\frac{1}{r} f_{t} \cos ^{2} \lambda_{21}}{1-\frac{1}{\pi r} f_{t} \cos \lambda_{21}}
$$

or:

$$
\sin \lambda_{21} \approx-\frac{f_{t}}{r} \cos ^{2} \lambda_{21}\left(1+f_{t} \frac{1}{\pi r} \cos \lambda_{21}\right)
$$

If $\theta$ close to 0 or 180 degree, assume $\cos \theta=1$. It becomes:

$$
\lambda_{21} \approx-\arcsin \left[\frac{f_{t}}{r}\left(1+f_{t} \frac{1}{\pi r}\right)\right]
$$

B. The second section

When the formulas 3.2 .31 and 3.2 .32 have the same $h$, that is the boundary of the two sections. 


$$
\begin{aligned}
& 2 f_{t} \sin \theta-\frac{2}{\pi r} f_{t}^{2} \sin \theta \cos \theta+\frac{4}{2 r} f_{t}^{2} \cos ^{2} \theta \\
& \left.=f_{t}\left[1-\frac{2 r_{o}}{\pi r} \sin \gamma\right)\right] \sin \theta-\frac{1}{\pi r} f_{t}^{2} \sin \theta \cos \theta+\frac{1}{2 r} f_{t}^{2} \cos ^{2} \theta+2 r_{o} \cos \gamma
\end{aligned}
$$

Solve the equation,

$$
\sin \lambda_{22}=\frac{2 r_{o} \cos \gamma-\frac{3}{2 r} f_{t}^{2} \cos ^{2} \lambda_{22}}{f_{t}\left(1+\frac{2 r_{o}}{\pi r} \sin \gamma\right)-\frac{1}{\pi r} f_{t}^{2} \cos \lambda_{22}}
$$

If $f_{t} / r \ll 1$ and $r_{o} / r<1$, it becomes:

$$
\lambda_{22}=\lambda_{1}=\arcsin \left(\frac{2 r_{o}}{f_{t}} \cos \gamma\right)
$$

\section{Cutting Force}

The cutting force can be derived by using the formulas 2.1.6 and 2.1.7, and cutting chip thickness formula 3.2.20 instead of the formula 2.1.3 (the third assumption of Tlusty's model).

$$
\begin{aligned}
d F_{x}= & -2 F_{u}\left[\left(1+(-1)^{z} \frac{2 r_{o}}{\pi r} \sin \gamma\right) \sin \theta-\frac{1}{\pi r} f_{t} \sin \theta \cos \theta+\frac{1}{2 r} f_{t} \cos ^{2} \theta-(-1)^{2} 2 \frac{r_{o}}{f_{t}} \cos \gamma\right] \\
& \times(\cos \theta d \theta+p \sin \theta d \theta) \\
d F_{y}= & 2 F_{u}\left[\left(1+(-1)=\frac{2 r_{o}}{\pi r} \sin \gamma\right) \sin \theta-\frac{1}{\pi r} f_{t} \sin \theta \cos \theta+\frac{1}{2 r} f_{t} \cos ^{2} \theta-(-1)^{2} 2 \frac{r_{o}}{f_{t}} \cos \gamma\right] \\
& \times(\sin \theta d \theta-p \cos \theta d \theta)
\end{aligned}
$$

Take the integration, 


$$
\begin{aligned}
& F_{x}=F_{u}\left\{\frac{1}{3} \frac{f_{t}}{r}\left(1+p \frac{2}{\pi}\right)\left(\sin ^{3} \theta_{e}-\sin ^{3} \theta_{s}\right)+\frac{1}{3} \frac{f_{t}}{r}\left(p-\frac{2}{\pi}\right)\left(\cos ^{3} \theta_{e}-\cos ^{3} \theta_{s}\right)\right. \\
& -\left[1+(-1)^{z} \frac{2 r_{o}}{\pi r} \sin \gamma\right]\left(\sin ^{2} \theta_{s}-\sin ^{2} \theta_{e}\right)+\frac{1}{2} p\left[1+(-1)^{2} \frac{2 r_{o}}{\pi r} \sin \gamma\right]\left(\sin 2 \theta_{s}-\sin 2 \theta_{e}\right) \\
& +\left[(-1)^{z} 4 \frac{r_{o}}{f_{t}} \cos \gamma-\frac{f_{t}}{r}\right]\left(\sin \theta_{e}-\sin \theta_{s}\right)-(-1)^{z} 4 p \frac{r_{o}}{f_{t}} \cos \gamma\left(\cos \theta_{e}-\cos \theta_{s}\right) \\
& \left.-p\left[1+(-1)^{z} \frac{2 r_{o}}{\pi r} \sin \gamma\right]\left(\theta_{e}-\theta_{s}\right)\right\} \\
& F_{y}=F_{u}\left\{\frac{1}{3} \frac{f_{t}}{r}\left(p-\frac{2}{\pi}\right)\left(\sin ^{3} \theta_{e}-\sin ^{3} \theta_{s}\right)-\frac{1}{3} \frac{f_{t}}{r}\left(1+p \frac{2}{\pi}\right)\left(\cos ^{3} \theta_{e}-\cos ^{3} \theta_{s}\right)\right. \\
& -p\left[1+(-1)^{z} \frac{2 r_{o}}{\pi r} \sin \gamma\right]\left(\sin ^{2} \theta_{s}-\sin ^{2} \theta_{e}\right)-\frac{1}{2}\left[1+(-1)=\frac{2 r_{o}}{\pi r} \sin \gamma\right]\left(\sin 2 \theta_{s}-\sin 2 \theta_{e}\right) \\
& +\left[(-1)^{2} 4 p \frac{r_{o}}{f_{t}} \cos \gamma-\frac{f_{t}}{r}\right]\left(\sin \theta_{e}-\sin _{s}\right)+(-1)^{2} 4 \frac{r_{o}}{f_{t}} \cos \gamma\left(\cos \theta_{e}-\cos \theta_{s}\right) \\
& \left.+\left[1+(-1)^{2} \frac{2 r_{o}}{\pi r} \sin \gamma\right]\left(\theta_{e}-\theta_{s}\right)\right\}
\end{aligned}
$$

(1) The cutting force of first cutter

In the formulas 3.2.41 and 3.2.42, let $z=0$. The first cutter cutting force is:

$$
\begin{aligned}
& F_{x}=F_{u}\left\{\frac{1}{3} \frac{f_{t}}{r}\left(1+p \frac{2}{\pi}\right)\left(\sin ^{3} \theta_{e}-\sin ^{3} \theta_{s}\right)+\frac{1}{3} \frac{f_{t}}{r}\left(p-\frac{2}{\pi}\right)\left(\cos ^{3} \theta_{e}-\cos ^{3} \theta_{s}\right)\right. \\
& -\left[1+\frac{2 r_{o}}{\pi r} \sin \gamma\right]\left(\sin ^{2} \theta_{s}-\sin ^{2} \theta_{e}\right)+\frac{1}{2} p\left[1+\frac{2 r_{o}}{\pi r} \sin \gamma\right]\left(\sin 2 \theta_{s}-\sin 2 \theta_{e}\right) \\
& +\left[4 \frac{r_{o}}{f_{t}} \cos \gamma-\frac{f_{t}}{r}\right]\left(\sin \theta_{e}-\sin \theta_{s}\right)-4 p \frac{r_{o}}{f_{t}} \cos \gamma\left(\cos \theta_{e}-\cos \theta_{s}\right) \\
& \left.-p\left[1+\frac{2 r_{o}}{\pi r} \sin \gamma\right]\left(\theta_{e}-\theta_{s}\right)\right\}
\end{aligned}
$$




$$
\begin{aligned}
& F_{y}=F_{u}\left\{\frac{1}{3} \frac{f_{t}}{r}\left(p-\frac{2}{\pi}\right)\left(\sin ^{3} \theta_{e}-\sin ^{3} \theta_{s}\right)-\frac{1}{3} \frac{f_{t}}{r}\left(1+p \frac{2}{\pi}\right)\left(\cos ^{3} \theta_{e}-\cos ^{3} \theta_{s}\right)\right. \\
& -p\left[1+\frac{2 r_{o}}{\pi r} \sin \gamma\right]\left(\sin ^{2} \theta_{s}-\sin ^{2} \theta_{e}\right)-\frac{1}{2}\left[1+\frac{2 r_{o}}{\pi r} \sin \gamma\right]\left(\sin 2 \theta_{s}-\sin 2 \theta_{e}\right) \\
& +\left[4 p \frac{r_{o}}{f_{t}} \cos \gamma-\frac{f_{t}}{r}\right]\left(\sin \theta_{e}-\sin \theta_{s}\right)+4 \frac{r_{o}}{f_{t}} \cos \gamma\left(\cos \theta_{e}-\cos \theta_{s}\right) \\
& \left.+\left[1+\frac{2 r_{o}}{\pi r} \sin \gamma\right]\left(\theta_{e}-\theta_{s}\right)\right\}
\end{aligned}
$$

or:

$$
F_{x}=F_{u}\left[C_{3} \frac{f_{t}}{r} \sin ^{3} \theta+C_{4} \frac{f_{t}}{r} \cos ^{3} \theta-\left(1+C_{5}\right) \sin ^{2} \theta+\frac{1}{2} p\left(1+C_{5}\right) \sin 2 \theta\right.
$$

$$
\left.\left.+\left(C_{6}-\frac{f_{t}}{r}\right) \sin \theta-p C_{6} \cos \theta-p\left(1+C_{5}\right) \theta\right]\right]_{\theta_{s}}^{\theta_{r}}
$$

$F_{y}=F_{u}\left[C_{4} \frac{f_{t}}{r} \sin ^{3} \theta-C_{3} \frac{f_{t}}{r} \cos ^{3} \theta-p\left(1+C_{5}\right) \sin ^{2} \theta-\frac{1}{2}\left(1+C_{5}\right) \sin 2 \theta\right.$

$$
\left.+p\left(C_{6}-\frac{f_{t}}{r}\right) \sin \theta+C_{6} \cos \theta+\left(1+C_{5}\right) \theta\right]\left.\right|_{\theta_{s}} ^{\theta_{e}}
$$

where: $C_{3}=\frac{1}{3}\left(1+p \frac{2}{\pi}\right)$

$$
\begin{aligned}
& C_{4}=\frac{1}{3}\left(p-\frac{2}{\pi}\right) \\
& C_{5}=\frac{2 r_{o}}{\pi r} \sin \gamma \\
& C_{6}=\frac{4 r_{o}}{f_{t}} \cos \gamma
\end{aligned}
$$


(2) The cutting force of second cutter

\section{A. The first section}

The cutting force can be derided by using formulas 2.1 .6 and 2.1.7, and cutting chip thickness formula 3.2.31.

$$
\begin{aligned}
& d F_{x}=-2 F_{u}\left(2 \sin \theta-\frac{2}{\pi r} f_{t} \sin \theta \cos \theta+\frac{4}{2 r} f_{t} \cos ^{2} \theta\right)(\cos \theta d \theta+p \sin \theta d \theta) \\
& d F_{y}=2 F_{u}\left(2 \sin \theta-\frac{2}{\pi r} f_{t} \sin \theta \cos \theta+\frac{4}{2 r} f_{t} \cos ^{2} \theta\right)(\sin \theta d \theta-p \cos \theta d \theta)
\end{aligned}
$$

Take the integration, the cutter cutting force is:

$$
\begin{aligned}
F_{x}=F_{u} & {\left[\frac{4}{3} \frac{f_{t}}{r}\left(1+\frac{p}{\pi}\right)\left(\sin ^{3} \theta_{e}-\sin ^{3} \theta_{s}\right)+\frac{4}{3} \frac{f_{t}}{r}\left(p-\frac{1}{\pi}\right)\left(\cos ^{3} \theta_{e}-\cos ^{3} \theta_{s}\right)-2\left(\sin ^{2} \theta_{e}-\sin ^{2} \theta_{s}\right)\right.} \\
& \left.+p\left(\sin 2 \theta_{e}-\sin 2 \theta_{s}\right)-\frac{4 f_{t}}{r}\left(\sin \theta_{e}-\sin \theta_{s}\right)-2 p\left(\theta_{e}-\theta_{s}\right)\right] \\
F_{y}=F_{u} & {\left[\frac{4}{3} \frac{f_{t}}{r}\left(p-\frac{1}{\pi}\right)\left(\sin ^{3} \theta_{e}-\sin ^{3} \theta_{s}\right)-\frac{4}{3} \frac{f_{t}}{r}\left(1+\frac{p}{\pi}\right)\left(\cos ^{3} \theta_{e}-\cos ^{3} \theta_{s}\right)-2 p\left(\sin ^{2} \theta_{e}-\sin ^{2} \theta_{s}\right)\right.} \\
& \left.-\left(\sin 2 \theta_{e}-\sin 2 \theta_{s}\right)-p \frac{4 f_{t}}{r}\left(\sin \theta_{e}-\sin \theta_{s}\right)+2\left(\theta_{e}-\theta_{s}\right)\right]
\end{aligned}
$$

or:

$$
\left.F_{x}=F_{u}\left[C_{7} \frac{f_{t}}{r} \sin ^{3} \theta+C_{8} \frac{f_{t}}{r} \cos ^{3} \theta-2 \sin ^{2} \theta+p \sin 2 \theta-\frac{4 f_{t}}{r} \sin \theta-2 p \theta\right]\right]_{\theta_{s}}^{\theta_{e}}
$$

$\left.F_{y}=F_{u}\left[C_{8} \frac{f_{t}}{r} \sin ^{3} \theta-C_{7} \frac{f_{t}}{r} \cos ^{3} \theta-2 p \sin ^{2} \theta-\sin 2 \theta-p \frac{4 f_{t}}{r} \sin \theta+2 \theta\right]\right]_{\theta_{s}}^{\theta_{e}}$

$$
\text { where : } \begin{aligned}
C_{7} & =\frac{4}{3}\left(1+\frac{p}{\pi}\right) \\
C_{8} & =\frac{4}{3}\left(p-\frac{1}{\pi}\right)
\end{aligned}
$$


B. The second section

The formulas 3.2.45 and 3.2.46 can be used to calculate the cutting force of the second section by using $-\mathrm{C}_{5}$ and $-\mathrm{C}_{6}$ instead of $\mathrm{C}_{5}$ and $\mathrm{C}_{6}$.

\subsection{Cutting Force Model of Conventional End Milling Operations}

That cutting force model has been derived above can be applied to all the cases of end milling operations. The conventional end milling operations is only a special case of the model, which can be simply obtained from the model. The cutting force expressions of the conventional end milling operations are much simpler than then the micro-end-milling operations.

\section{Cutting Force Model of Conventional End Milling Operations without Tool Run-} out

The conventional tools cutting force model can be simply obtained from the cutting force model by considering $\mathrm{f}_{\mathrm{t}} / \mathrm{r}=0$ and $\mathrm{r}_{\mathrm{o}} / \mathrm{r}=0$.

From the formulas 3.1.28 and 3.1.29, the cutting force model becomes:

$$
\begin{aligned}
& F_{x}=\left.F_{u}\left[-\sin ^{2} \theta+\frac{1}{2} p \sin 2 \theta-p \theta\right]\right|_{\theta_{s}} ^{\theta_{e}} \\
& F_{y}=\left.F_{u}\left[-p \sin ^{2} \theta-\frac{1}{2} \sin 2 \theta+\theta\right]\right|_{\theta_{s}} ^{\theta_{e}}
\end{aligned}
$$

This is exact Tlusty's cutting force model. 


\section{Cutting Force Model of Conventional End Milling Operations with Tool Run-out}

In the two-flute tool case, the cutting force model can be derived from formulas

$3.2 .45,3.2 .46,3.2 .51$ and 3.2 .52 by considering $\mathrm{f}_{\mathrm{t}} / \mathrm{r}=0$.

For the first cutter,

$$
\begin{aligned}
& F_{x}=\left.F_{u}\left[-\sin ^{2} \theta+\frac{1}{2} p \sin 2 \theta+\frac{4 r_{o}}{f_{t}} \cos \gamma(\sin \theta-p \cos \theta)-p \theta\right]\right|_{\theta_{s}} ^{\theta_{e}} \\
& F_{y}=\left.F_{u}\left[-p \sin ^{2} \theta-\frac{1}{2} \sin 2 \theta+\frac{4 r_{o}}{f_{t}} \cos \gamma(p \sin \theta+\cos \theta)+\theta\right]\right|_{\theta_{s}} ^{\theta_{e}}
\end{aligned}
$$

For the first section of the second cutter,

$$
\underline{F_{x}=\left.F_{u}\left[-2 \sin ^{2} \theta+p \sin 2 \theta-2 p \theta\right]\right|_{\theta_{s}} ^{\theta_{e}}}
$$

$F_{y}=\left.F_{u}\left[-2 p \sin ^{2} \theta-\sin 2 \theta+2 \theta\right]\right|_{\theta_{s}} ^{\theta_{e}}$

For the second section of the second cutter,

$$
\begin{aligned}
& F_{x}=\left.F_{u}\left[-\sin ^{2} \theta+\frac{1}{2} p \sin 2 \theta-\frac{4 r_{o}}{f_{t}} \cos \gamma(\sin \theta-p \cos \theta)-p \theta\right]\right|_{\theta_{s}} ^{\theta_{e}} \\
& F_{y}=\left.F_{u}\left[-p \sin ^{2} \theta-\frac{1}{2} \sin 2 \theta-\frac{4 r_{o}}{f_{t}} \cos \gamma(p \sin \theta+\cos \theta)+\theta\right]\right|_{\theta_{s}} ^{\theta_{e}}
\end{aligned}
$$

The integrating angle can be got from the formula 3.2.34.

$$
\lambda_{1}=\arcsin \left(\frac{2 r_{o}}{f_{t}} \cos \gamma\right)
$$




\section{Chapter IV}

\section{Model Based Cutting Force Characteristics and Surface Finish}

Model based cutting force characteristics of micro-end-milling operations are discussed in this chapter. The cutting force performances of ten different variables (spindle speed, feed rate, depth of cut, tool run-out and its angle, tool diameter, helix angle and the numbers of flutes) are presented. The calculation formulas of work-piece surface roughness and precision have been derived from the tool cutting edge tip profile equations of the analytical model.

\subsection{Cutting Force Profiles}

The developed analytical cutting force model conventional milling (see Chapter III) can be used to estimate the cutting force of end milling operations. Six sample cases, which include two-flute and four-flute tool climbing and conventional milling with and without tool run-out cases are presented in Figure 4.1 to 4.6. In the sample cases, 0.020 " diameter tool with 45 degree helix angle, 15,000 rpm spindle speed, $70 \mathrm{ipm}$ feed rate, 0.010 inch depth of cut, $50 \%$ overlapping and $130,000 \mathrm{~N} /$ inch $^{2}$ material coefficient are selected. If the case is a tool run-out case, 0.001 " tool run-out with 60 -degree run-out angle are chosen. They are listed as below:

Case 1: Two-flute tool, climbing milling without run-out (Figure 4.1).

Case 2: Two-flute tool, conventional milling without run-out (Figure 4.2). 
Case 3: Two-flute tool, climbing milling with run-out (Figure 4.3).

Case 4: Two-flute tool, conventional milling with run-out (Figure 4.4).

Case 5: Four-flute tool, climbing milling without run-out (Figure 4.5).

Case 6: Four-flute tool, conventional milling without run-out (Figure 4.6).

In all these sample cases the tool wear is not considered, which will be discussed in the following chapter.

From the sample cases 1 and 2 (see Figure 4.1 and 4.2), it is known that the maximum resultant cutting force of climbing milling and conventional milling are almost in the same level. If the effect of feed rate is not considered, the cutting force of the thrust direction is larger than the feed direction in the climbing milling, and the cutting force of the feed and thrust direction are almost the same in the conventional milling.

In the conventional milling, the angle of the maximum resultant cutting force is always the exit cutting angle, which is independent of the spindle speed, feed rate and depth of cut. It is 90 degree in the 50\% overlapping case. In climbing milling, the angle of the maximum resultant cutting force only depends on the depth of cut, which is b / ( $\mathrm{t} \tan$ $\gamma)$ in the case of $50 \%$ overlapping. It is $147(90+57)$ degree in the sample case.

A very little change in run-out would produce a $25 \%$ change in the maximum resultant cutting force (compare Figure 4.3 and 4.4 run-out cases with Figures 4.1 and 4.2 without run-out cases). If the tool run-out is larger than the complete run-out that can be calculated by the formula 4.2 .2 (see next section of the chapter), the maximum resultant cutting force is no more increased even when the tool run-out is increased. In this case it becomes a one-flute cutting. 


\section{Cutting Force of Climbing Milling without Tool Run-out}

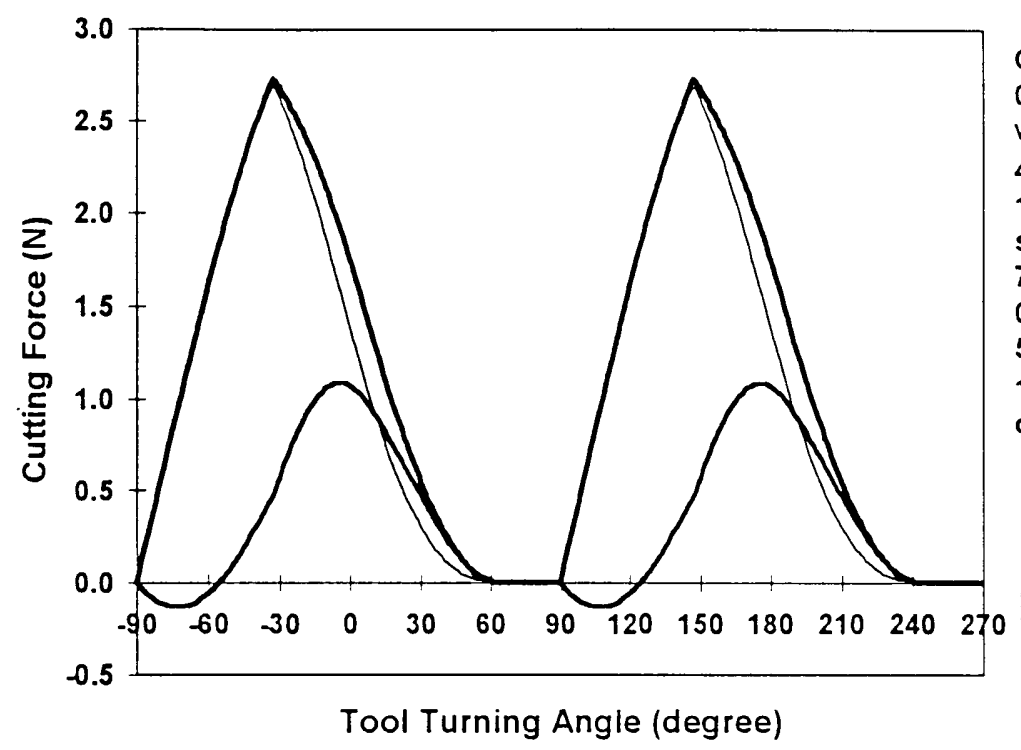

Operation Condition: 0.020 " diameter tool with two flutes and $45^{\circ}$ helix angle. $15,000 \mathrm{rpm}$ spindle speed.

$70 \mathrm{ipm}$ feed rate. 0.010 " depth of cut. $50 \%$ overlapping. $130,000 \mathrm{~N} / \mathrm{in} .^{2}$ material coefficent.

Figure 4.1 Cutting force of two-flute tool, climbing milling without tool run-out

\section{Cutting Force of Conventional Milling without Tool Run-out}

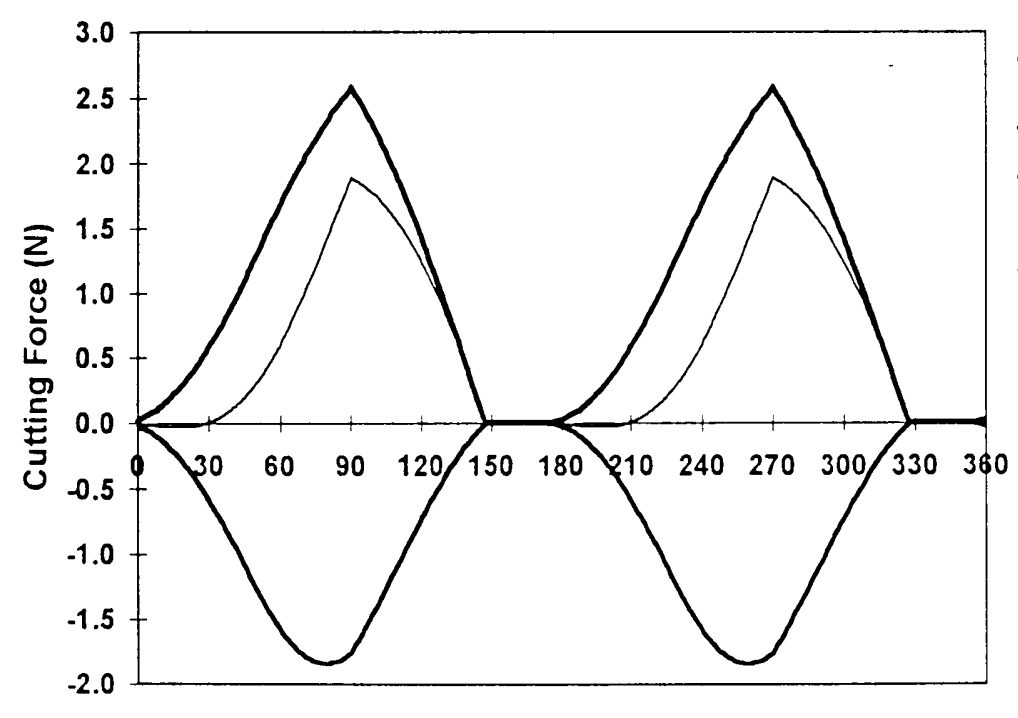

Operation Condition: 0.020 " diameter tool with two flutes and $45^{\circ}$ helix angle. $15,000 \mathrm{rpm}$ spindle speed.

$70 \mathrm{ipm}$ feed rate. 0.010 " depth of cut. $50 \%$ overlapping. 130,000 N/in. ${ }^{2}$ material coefficent.

Tool Turning Angle (degree)

Figure 4.2 Cutting force of two-flute tool, conventional milling without tool run-out 


\section{Cutting Force of Climbing Milling with Tool Run-out}

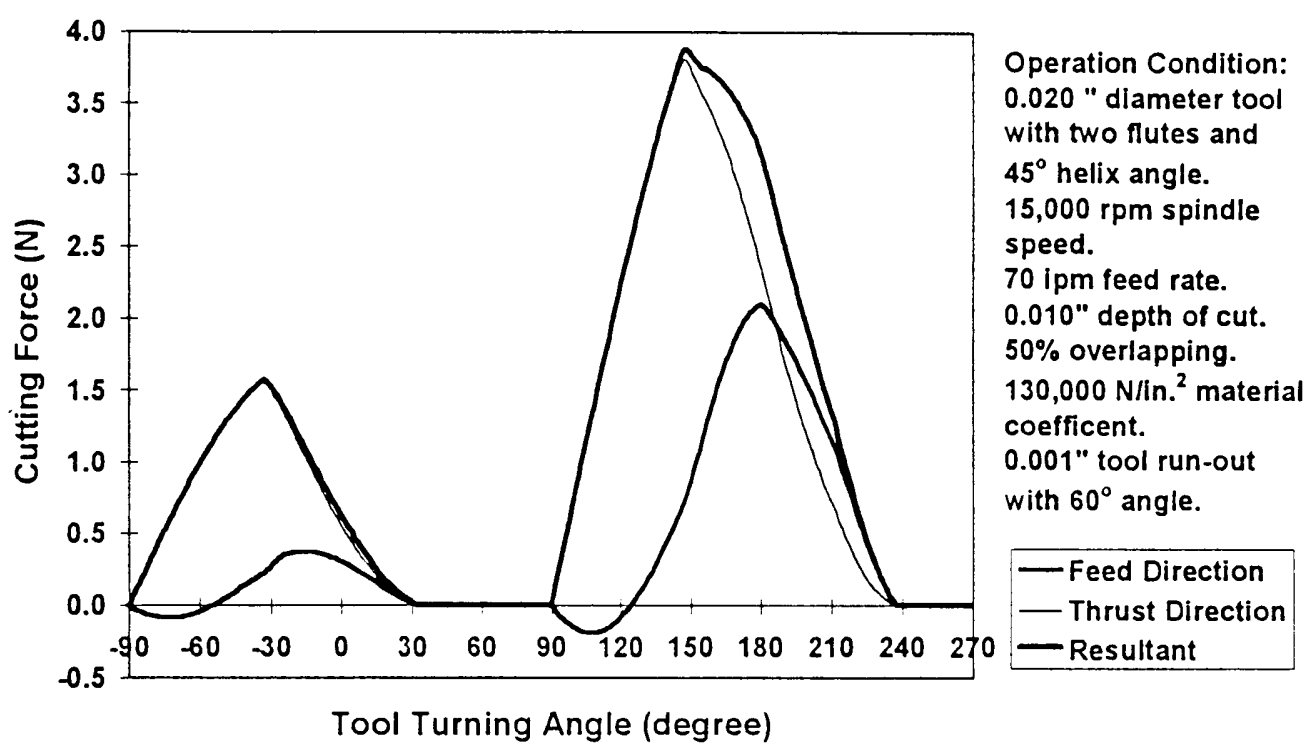

Figure 4.3 Cutting force of two-flute tool, climbing milling with tool run-out

\section{Cutting Force of Conventional Milling with Tool Run-out}

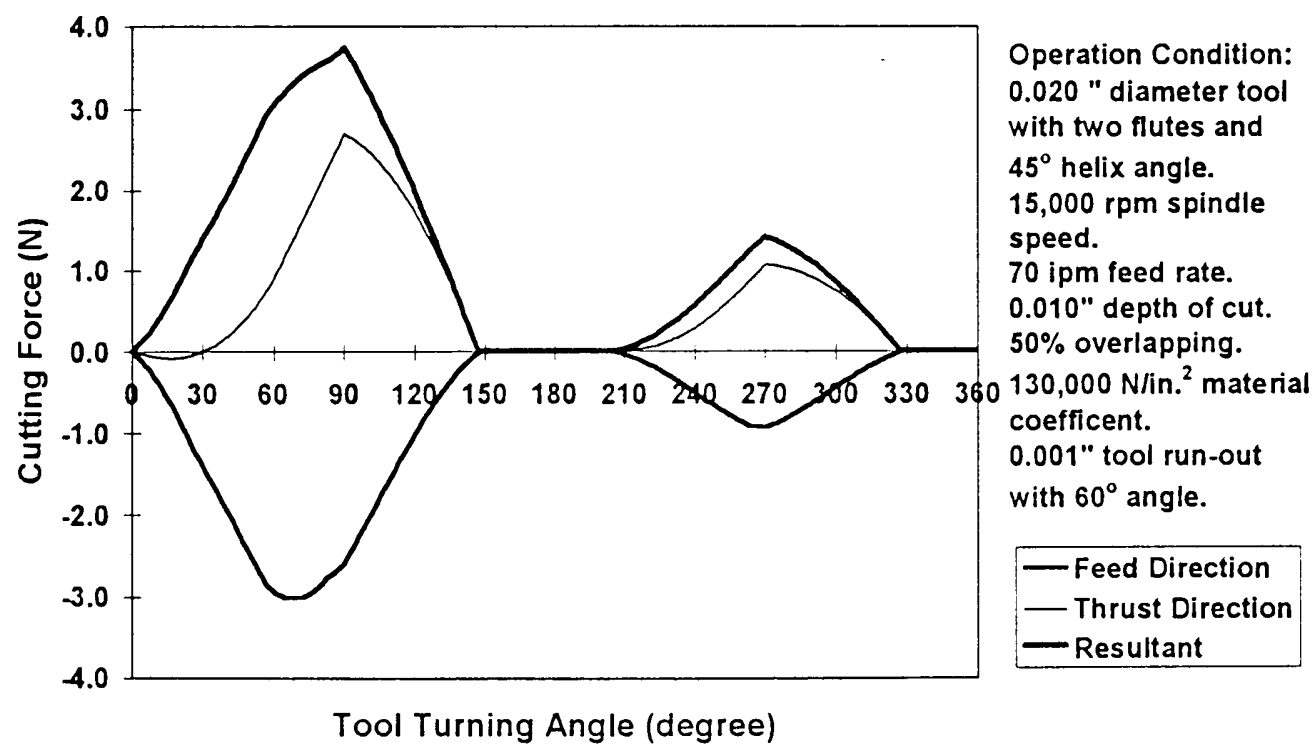

Figure 4.4 Cutting force of two-flute tool, conventional milling with tool run-out 


\section{Cutting Force of Climbing Milling without Tool Run-out}

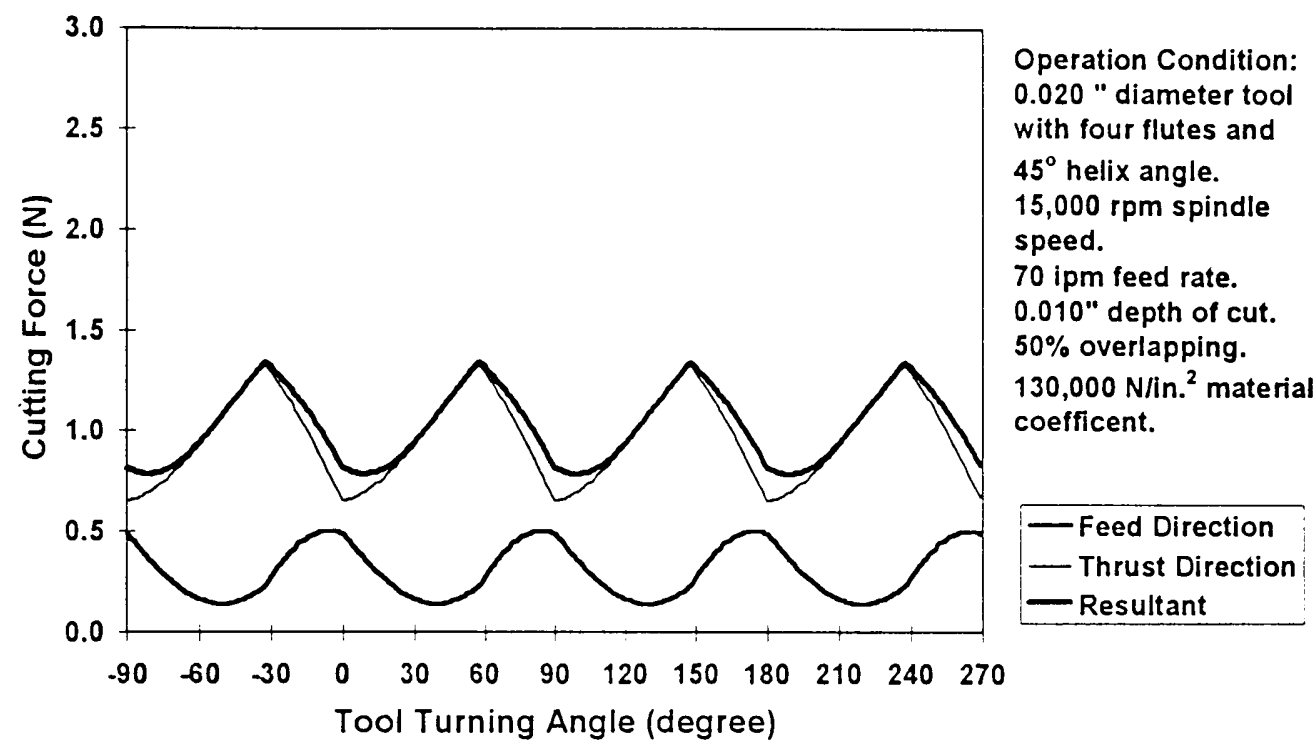

Figure 4.5 Cutting force of four-flute tool, climbing milling without tool run-out

\section{Cutting Force of Conventional Milling without Tool Run-out}

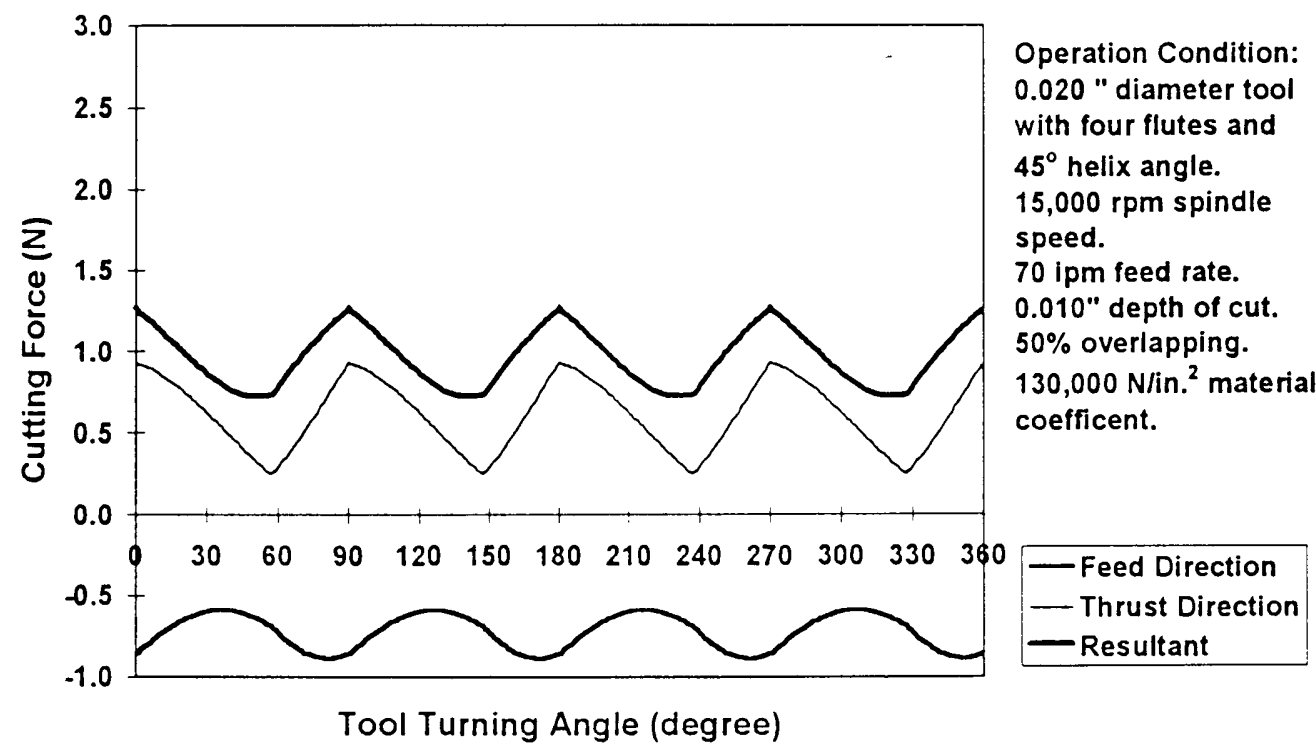

Figure 4.6 Cutting force of four-flute tool, conventional milling without tool run-out 
The feed and thrust direction cutting force can be used to decide the tool run-out and its angle, which will be discussed in Chapter V.

The maximum resultant cutting force of the four-flute tools (see Figure 4.5 and 4.6) is only half of the two-flute tools (see Figure 4.1 and 4.2). The cutting force oscillation of the four-flute tools becomes only one forth of the two-flute tools.

\subsection{Cutting Force Characteristics}

The maximum cutting force of tools is the most important factor in the micro-endmilling operations. It can be used to indicate tool wear because it gradually increases when the tool is worn, which is the most important reason of the tool breakage. It has been proved in many experimental cases of the micro-end-milling operations (see Chapter V). The only cutting force in the thrust direction of the micro-end-milling operations is chosen for the studies because the cutting force in the feed direction has very similar characteristics in most cases.

A special case is selected for the studied. All other cases discussed in the chapter is around this special case with different parameter changes. In the studied case, two-flute 20 " diameter tool with a 45 degree helix angle are considered working in $50 \%$ overlapped climbing end milling operations without tool run-out and wear. The working conditions are selected as $15,000 \mathrm{rpm}$ spindle speed, $70 \mathrm{ipm}$ feed rate and 0.030 " depth of cut. The material coefficient is considered as $130,000 \mathrm{~N} /$ inch $^{2}$. The conditions will be used in all the discussed case without other explanations. 


\section{Cutting Force Characteristics with Working Conditions}

Three most interesting working conditions, spindle speed, feed rate and depth of cut, are studied. The spindle speeds are selected between 5,000 and 45,000 rpm, feed rates between 20 and $120 \mathrm{ipm}$, depth of cut between 0.010 and $0.030 \mathrm{inch}$. The tools used in the studies are two-flute $0.010 ", 0.020$ " and 0.030 " micro-end-mills.

\section{- Cutting force characteristics with spindle speed:}

The spindle speeds from 5,000 to 45,000 rpm have been studied. Three different feed rates $(20,70$ and $120 \mathrm{ipm})$ and tool diameters (0.010", 0.020 " and $0.030 ")$ are selected in the study. The cutting force is decreased when spindle speed is increased. It can be divided into two sections, in which the cutting force is decreased sharply from $5,000 \mathrm{rpm}$ to a certain spindle speed and gently after this speed. This speed called critical spindle speed depends on the feed rate and tool diameter. In the study case, it can be approximated that critical spindle speed is around $1,000 \mathrm{rpm}$ in the $20 \mathrm{ipm}$ feed rate case, $2,000 \mathrm{rpm}$ in the $70 \mathrm{ipm}$ case and 3,000 rpm in the $120 \mathrm{ipm}$ case from Figure 4.7. It does not have much help for micro-end-milling operations to increase the spindle speed after the critical point. For different tool diameters, the same conclusion can be obtained from Figure 4.8. The larger tool diameter becomes, the smaller critical spindle speed is got and the littler cutting force is decreased.

The critical spindle speed obtained from the cutting force model can be used to select the spindle speed of micro-end-milling operations. 


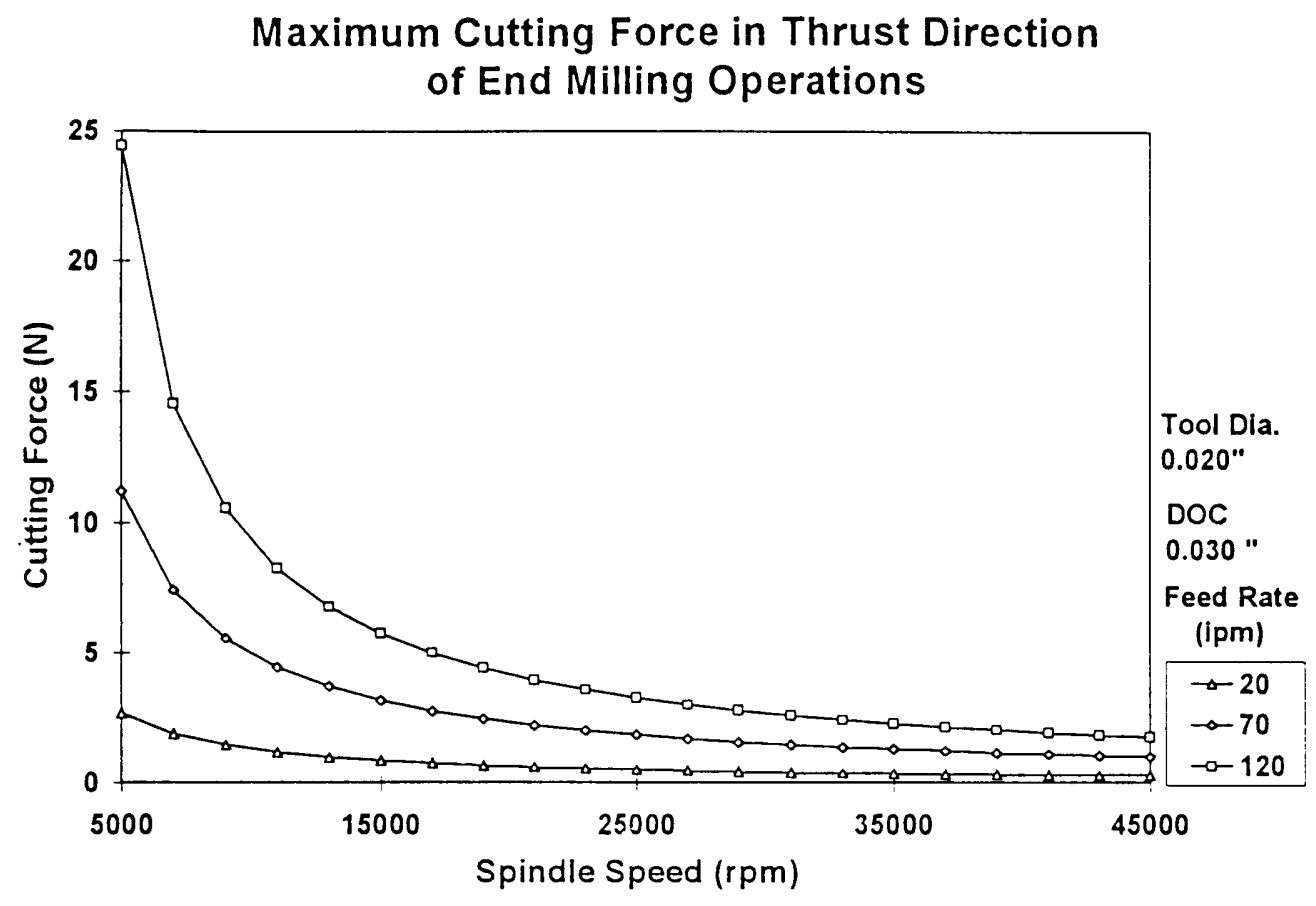

Figure 4.7 Cutting force characteristics with spindle speed
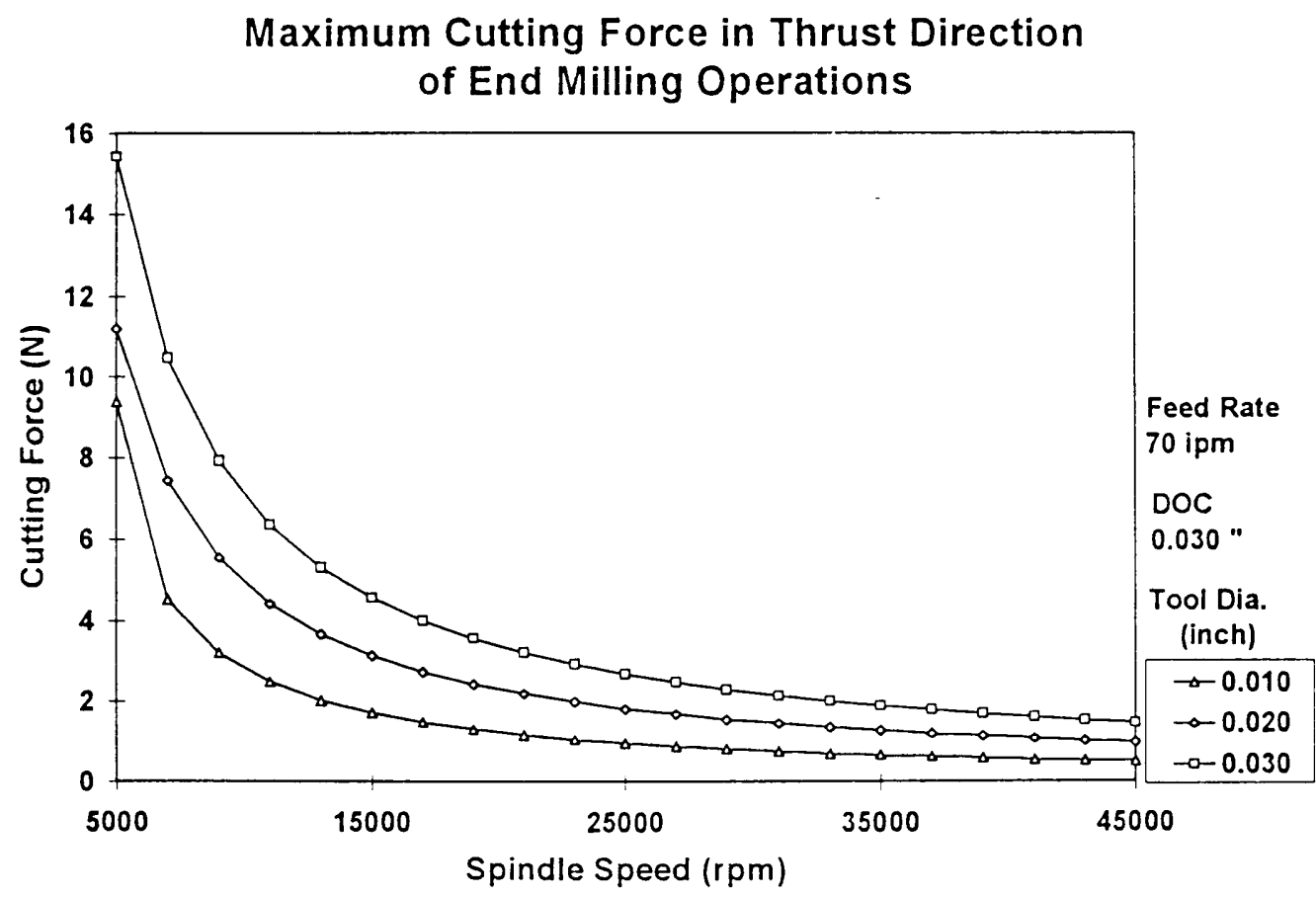

Figure 4.8 Cutting force characteristics with spindle speed 


\section{- Cutting force characteristics with feed rate:}

The feed rates from 20 to $120 \mathrm{ipm}$ have been studied. Three different spindle speeds $(15,000,30,000$ and $45,000 \mathrm{rpm})$ are selected in the study. The cutting force is increased linearly when feed rate is increased. The increasing slope of the cutting force depends on the selected spindle speed. When spindle speed is turned to faster, it becomes smaller and the cutting force increase becomes gentler (see Figure 4.9).

Increasing spindle speed and decreasing feed rate can reduce the cutting force. It is efficient to reduce the cutting force by increasing spindle speed when it is lower than the critical spindle speed. Otherwise, it becomes better by reducing feed rate.

The cutting force will be unchanged if the ratio of feed rate to the spindle speed is a constant (see Figure 4.11 and 4.12).

\section{Maximum Cutting Force in Thrust Direction of End Milling Operations}

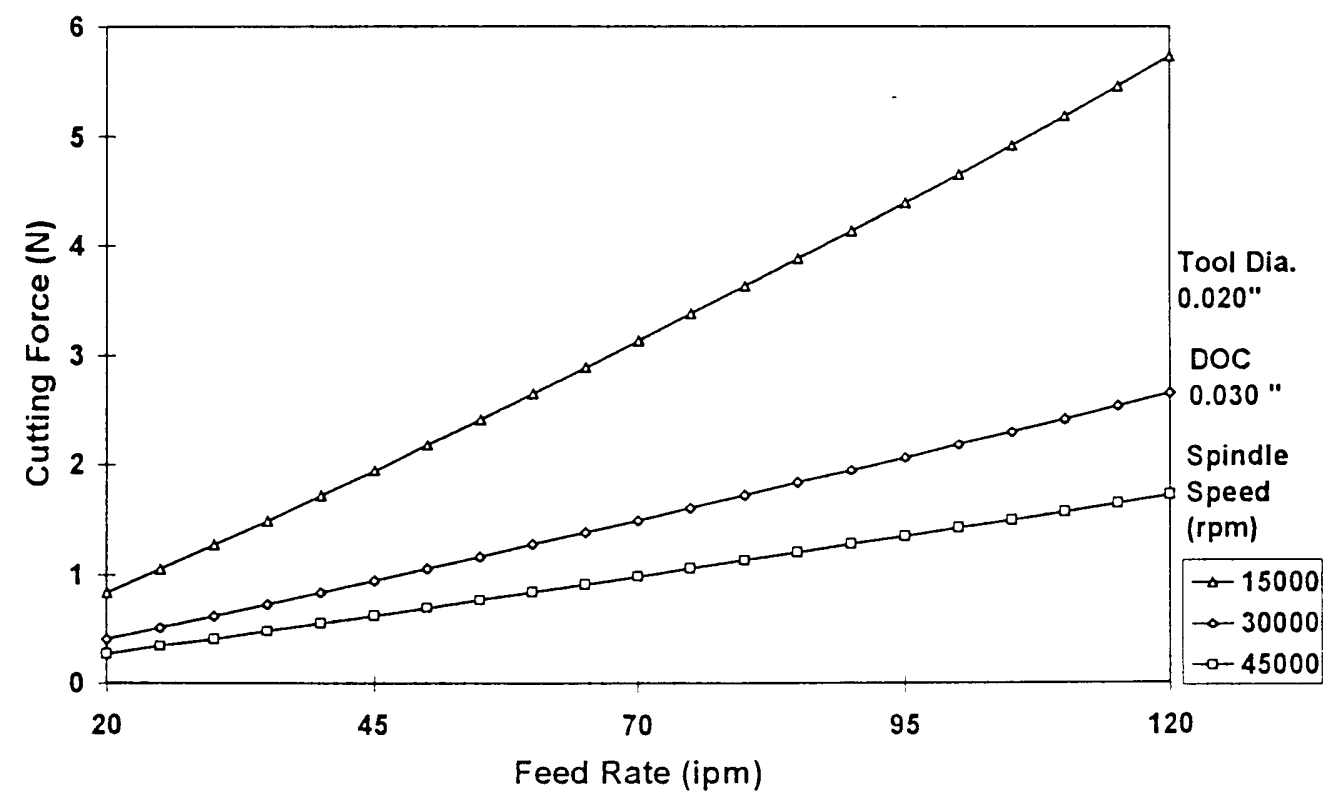

Figure 4.9 Cutting force characteristics with feed rate 
- Cutting force characteristics with depth of cut:

The depths of cut from 0 to 0.050 " have been studied. Three different spindle speeds $(15,000,30,000$ and $45,000 \mathrm{rpm})$ are selected in the study. When depth of cut is increased, the cutting force is increased up to a certain level then kept there independent of the depth of cut (see Figure 4.10, 4.13 to 4.16 ).

The critical depth of cut depends on the cutting angle of the work-piece and tool helix angle. It can be obtained from the analytical model. If the depth of cut is less then $\pi \mathrm{r}$, which happens in most micro-end-milling, it can be calculated by following formula.

$$
b_{c}=\frac{r \varphi}{\tan \gamma}
$$

\section{Maximum Cutting Force in Thrust Direction of End Milling Operations}

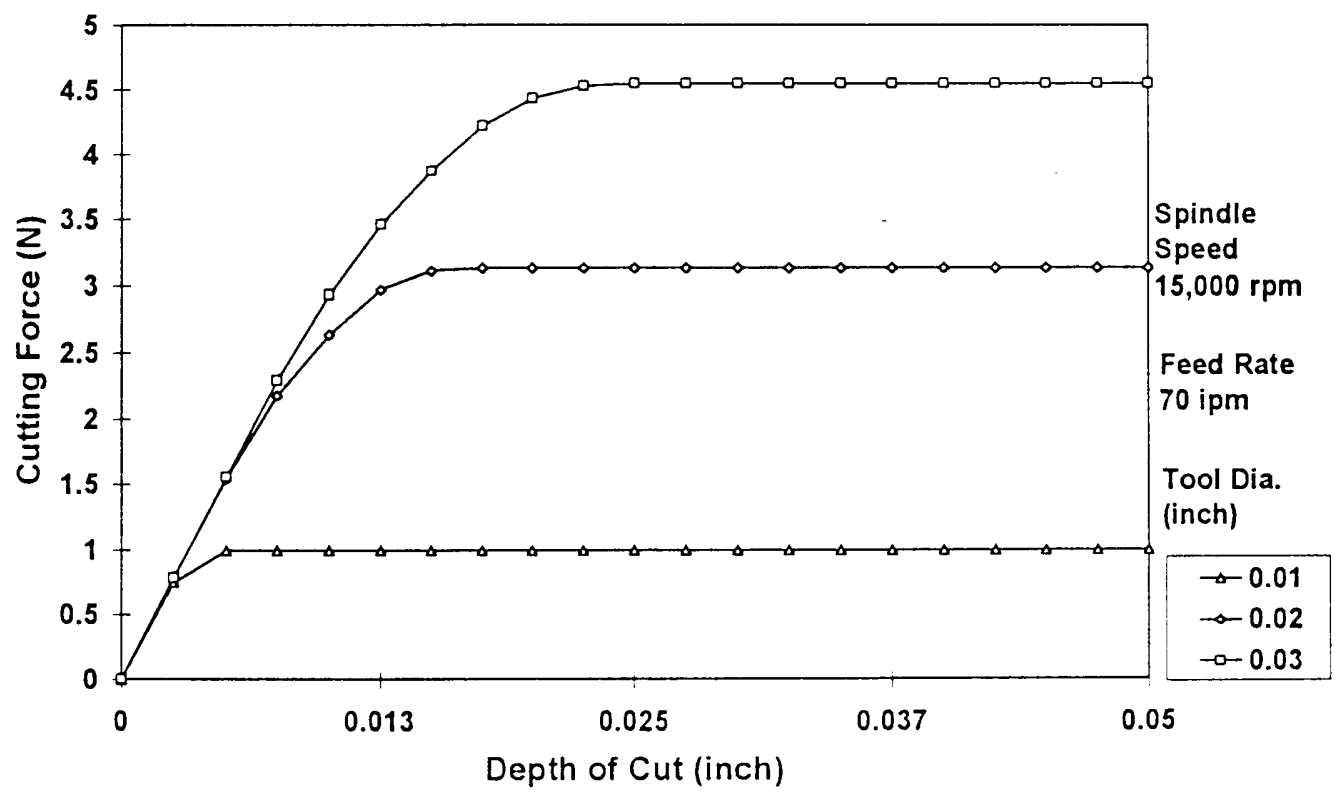

Figure 4.10 Cutting force characteristics with depth of cut

The three dimensional cutting force graphics are presented in Figure 4.11 to 4.16. 


\section{Maximum Cutting Force in Thrust Direction of End Milling Operations}
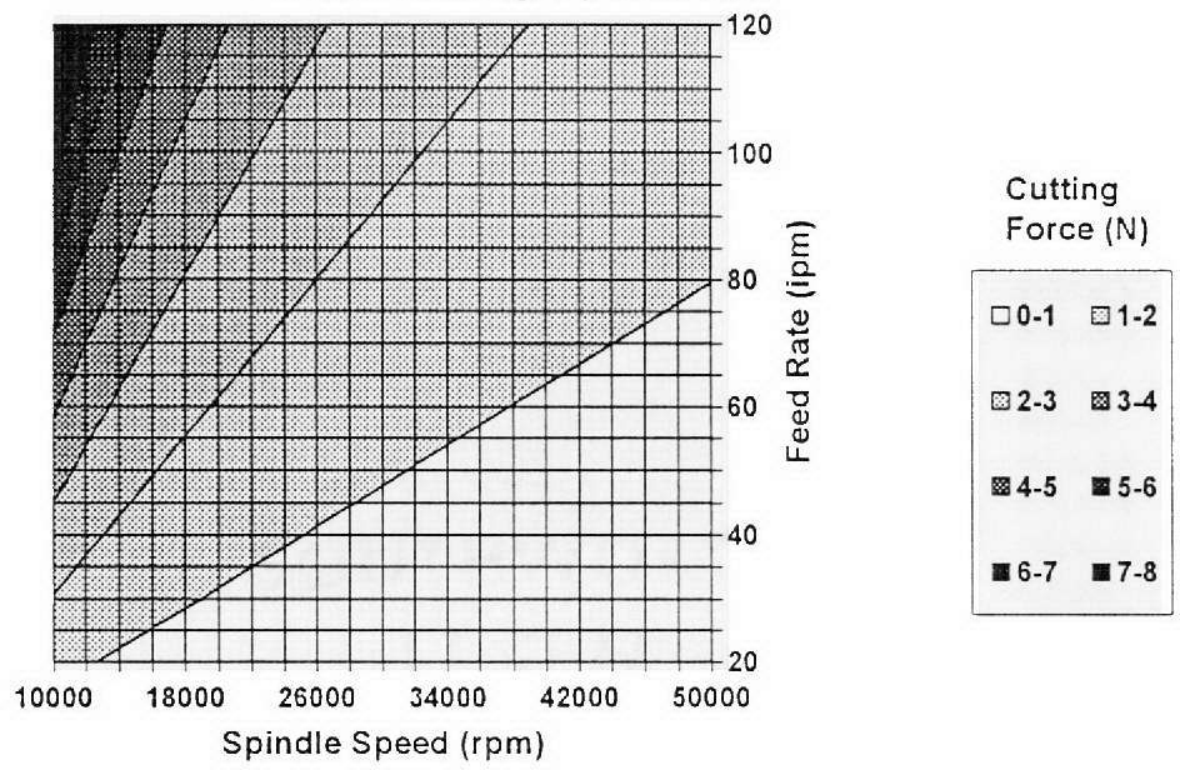

Figure 4.112-D cutting force characteristics with spindle speed and feed rate

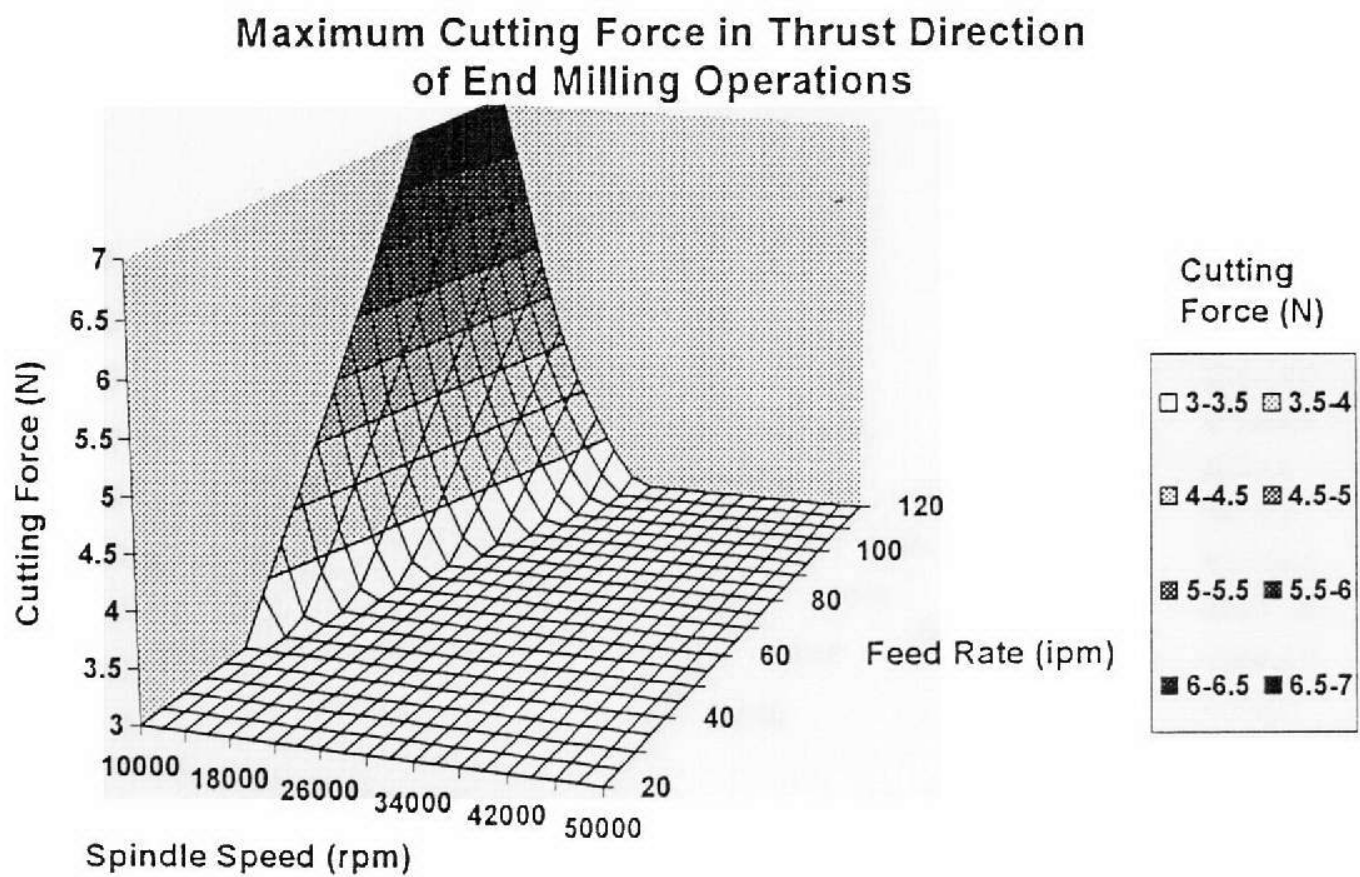

Figure 4.12 3-D cutting force characteristics with spindle speed and feed rate 


\section{Maximum Cutting Force in Thrust Direction of End Milling Operations}

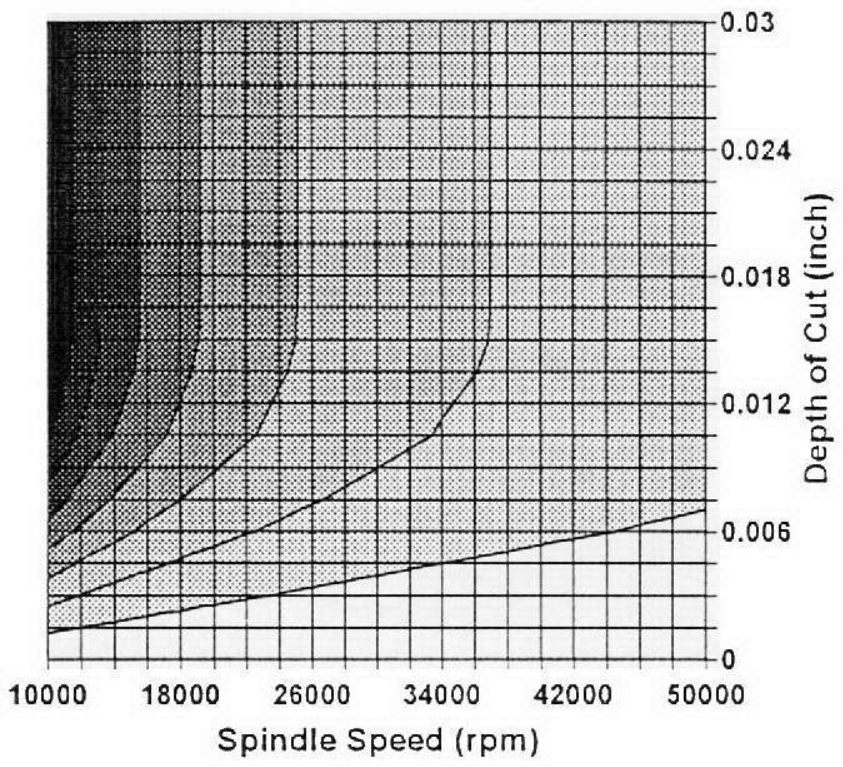

Cutting

Force (N)

\begin{tabular}{|c|}
\hline$=4.24 .8$ \\
\hline$=3.6-4.2$ \\
\hline 3-3.6 \\
\hline ख $2.4-3$ \\
\hline $1.8-2.4$ \\
\hline 圈 1.2-1.8 \\
\hline$\square 0.6-1.2$ \\
\hline$\square 0-0.6$ \\
\hline
\end{tabular}

Figure 4.13 2-D cutting force characteristics with spindle speed and depth of cut

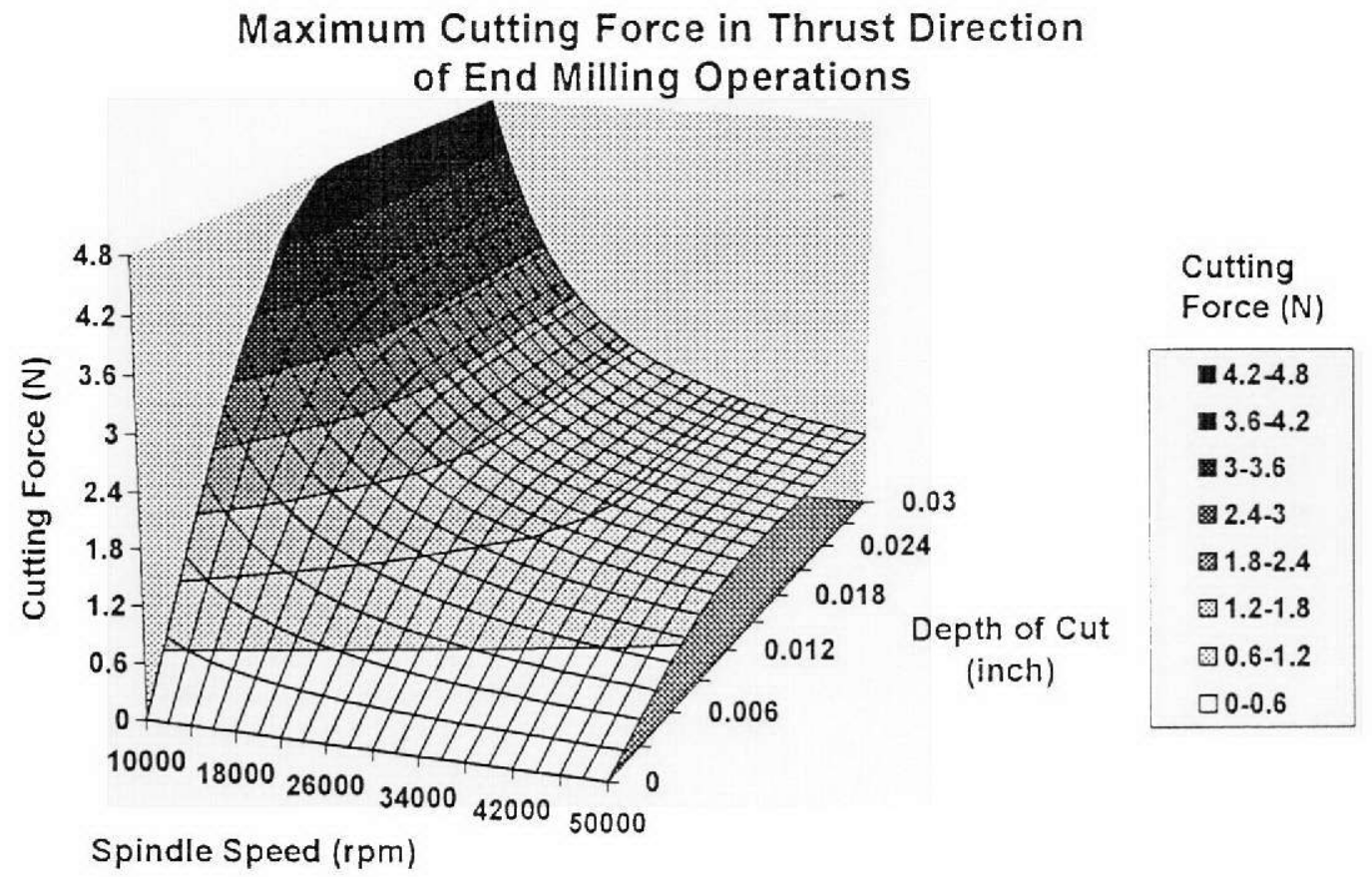

Figure 4.14 3-D cutting force characteristics with spindle speed and depth of cut 
Maximum Cutting Force in Thrust Direction of End Milling Operations

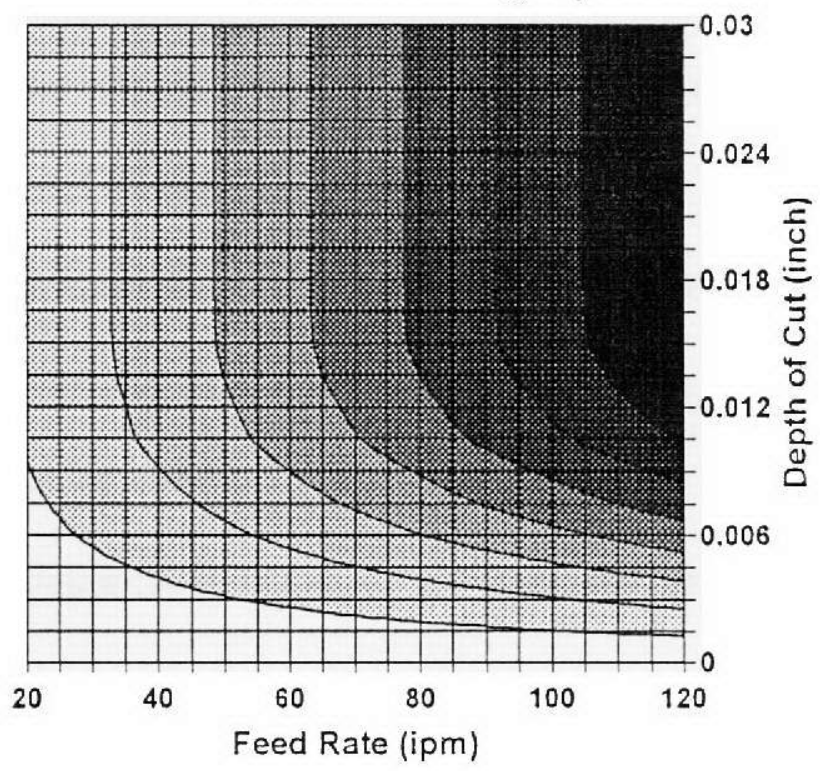

Cutting Force (N)

4 4.9-5.6

= $4.2-4.9$

3.5-4.2

잉 2.8-3.5

2.1-2.8

중.4-2.1

0.7-1.4

$\square 0-0.7$

Figure 4.15 2-D cutting force characteristics with feed rate and depth of cut

\section{Maximum Cutting Force in Thrust Direction of End Milling Operations}

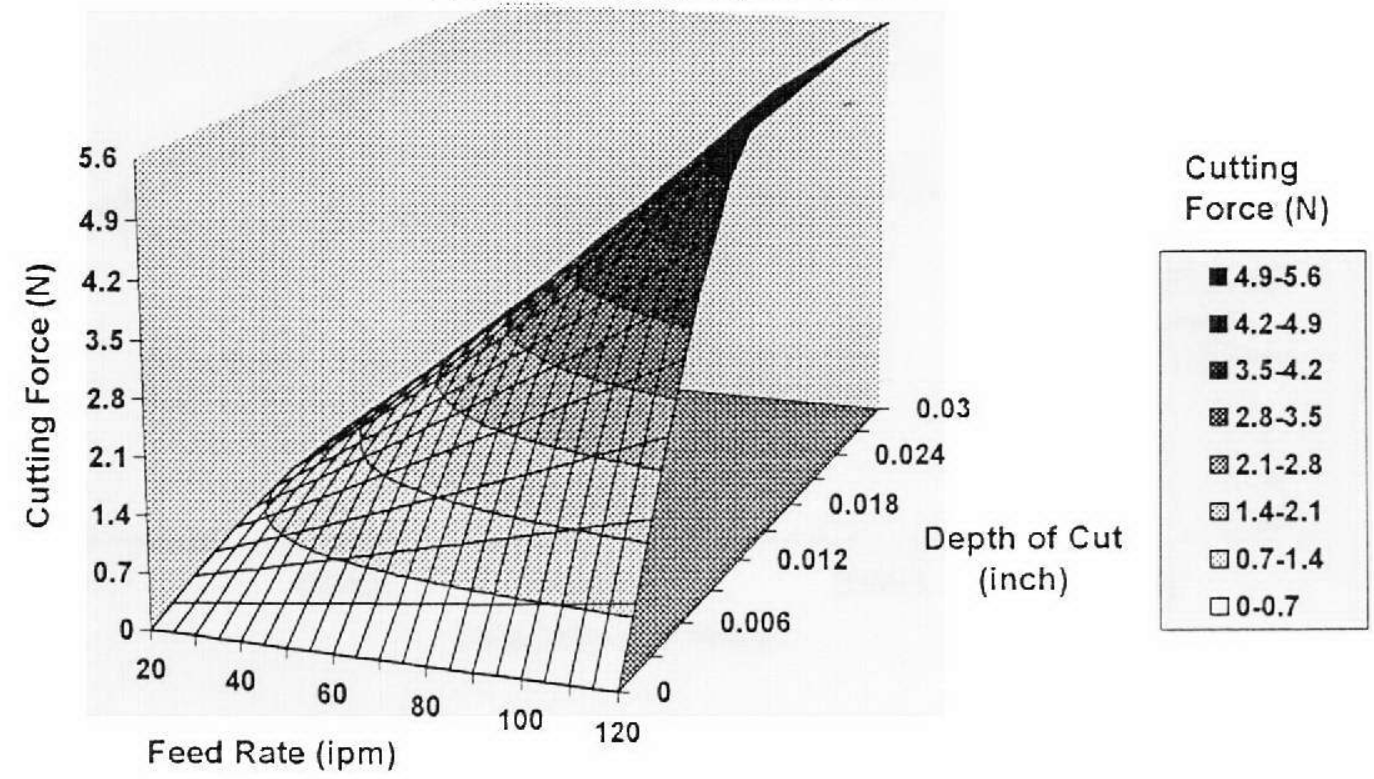

Figure 4.16 3-D cutting force characteristics with feed rate and depth of cut 


\section{Cutting Force Characteristics with Tool Run-out}

- Cutting force characteristics with tool run-out:

The tool run-out from 0 to 0.020 " has been studied. Three different tool run-out angles $(0,45$ and 90 degree $)$ are selected in the study. The cutting force is increased when tool run-out is increased. Usually, except the case of 90 degree run-out angle, it can be divided into two sections, in which the cutting force is increased linearly to a maximum tool run-out called complete run-out then kept there. When tool with 90-degree run-out angle, the cutting force is increased very little (see Figure 4.17).

\section{Maximum Cutting Force in Thrust Direction of End Milling Operations}

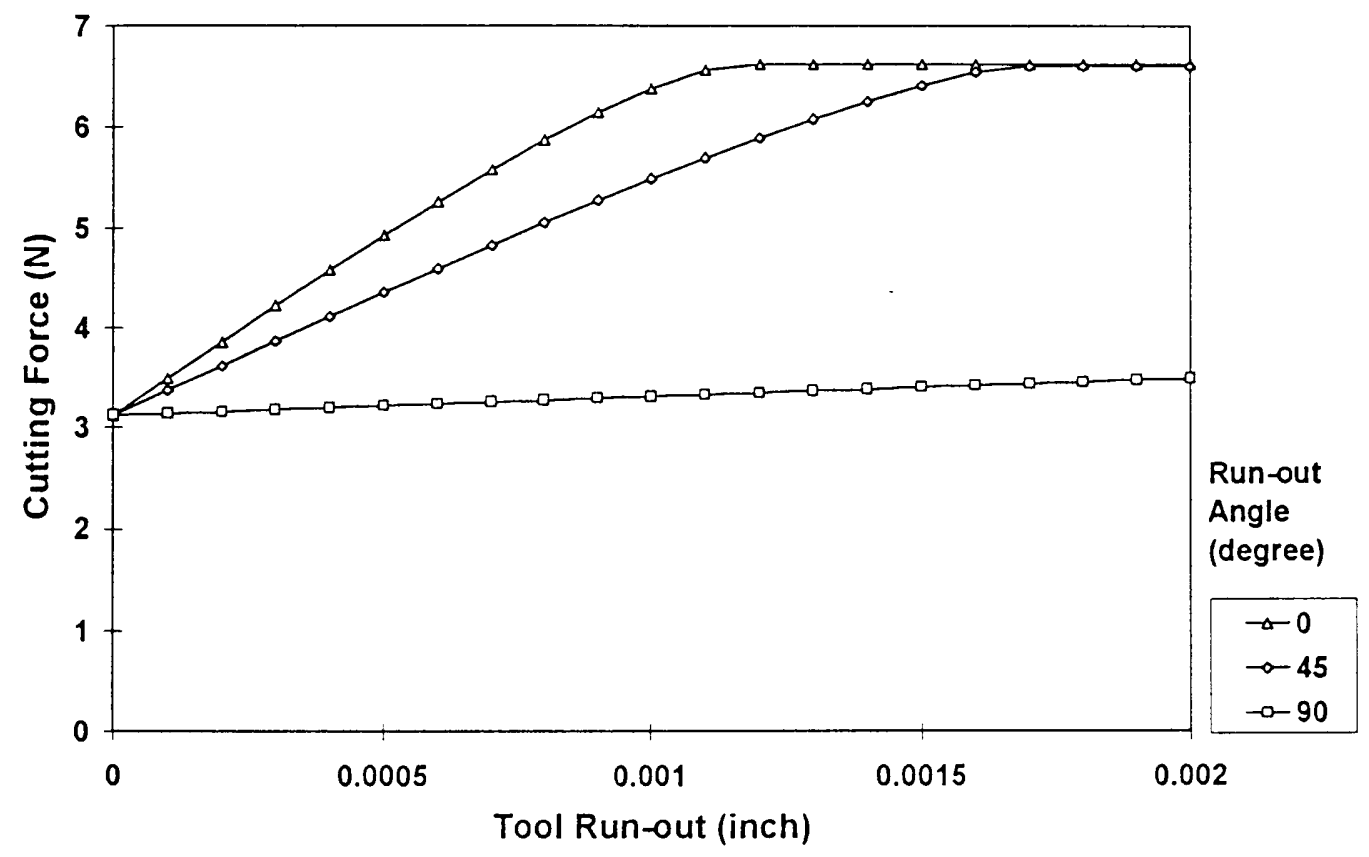

Figure 4.17 Cutting force characteristics with tool run-out 
The physical meaning of the complete tool run-out is that only one of the tool flutes works when the tool run-out is larger than it. For two-flute tool, the complete runout depends on the tool run-out angle and feed per tooth. It can be derived from the analytical model.

$$
R o c=\frac{f_{t}}{2 \cos \gamma}
$$

In the study cases, the complete tool run-out have be calculated, which is 0.0012 inch in the 0 degree run-out angle case and 0.00165 inch in the 45 degree case.

\section{- Cutting force characteristics with tool run-out angle:}

The tool run-out angles from 0 to 90 degree have been studied. The cutting force is decreased when the tool run-out angle is changed from parallel to the tool cutting edges ( 0 degree) to perpendicular to them ( 90 degree). When the tool run-out angle is closely perpendicular to the tool cutting edges, the cutting force has a minimum value. When the tool run-out angle is parallel to the tool cutting edges, the cutting force has a maximum value that almost double of the minimum value (see Figure 4.18).

From cutting force model, it has been proved that cutting force caused by the tool run-out can be minimized by setting the tool run-out perpendicular to the tool cutting edges. For normal tool holders, screwing the tool perpendicularly to the tool cutting edges can easily do it. In the view of the work-piece precision, the same conclusion can be obtained from exactly computed results, which will be discussed in the following section of this chapter. 


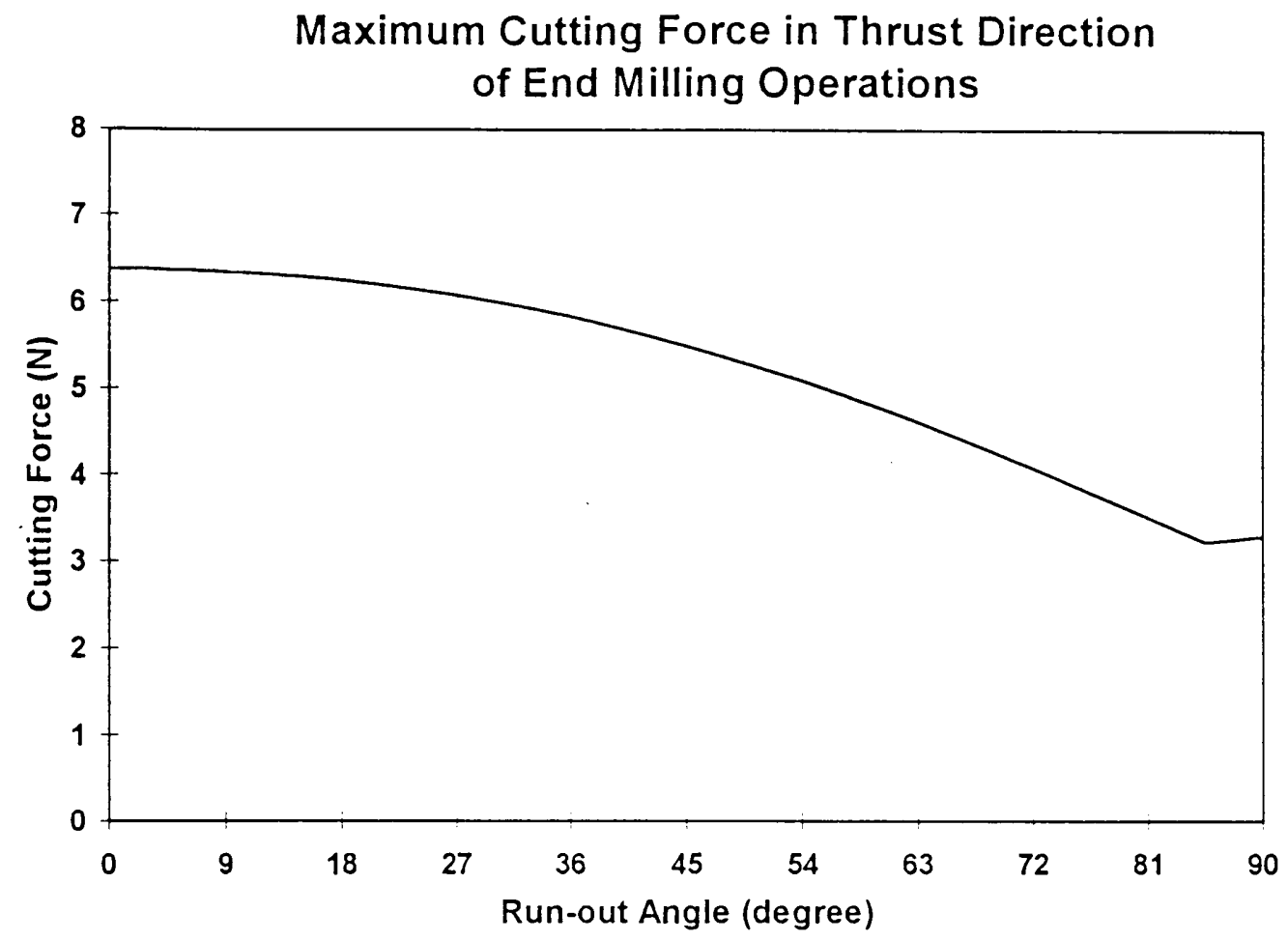

Figure 4.18 Cutting force characteristics with tool run-out angle

The three-dimensional graphics of the cutting force with tool run-out and run-out angle are presented in Figure 4.19 and 4.20. It can be known that tool run-out and angle can not be recognized by only one maximum cutting force value because the maximum cutting force is a multiple value function of the tool run-out and its angle. If it is tried to estimate the tool run-out and its angle, the maximum cutting force of both feed and thrust directions have to be considered. The tool run-out and its angle are a single value function of the maximum cutting force of both feed and thrust directions. The detail will be discussed in Chapter V. 
Maximum Cutting Force in Thrust Direction of End Milling Operations
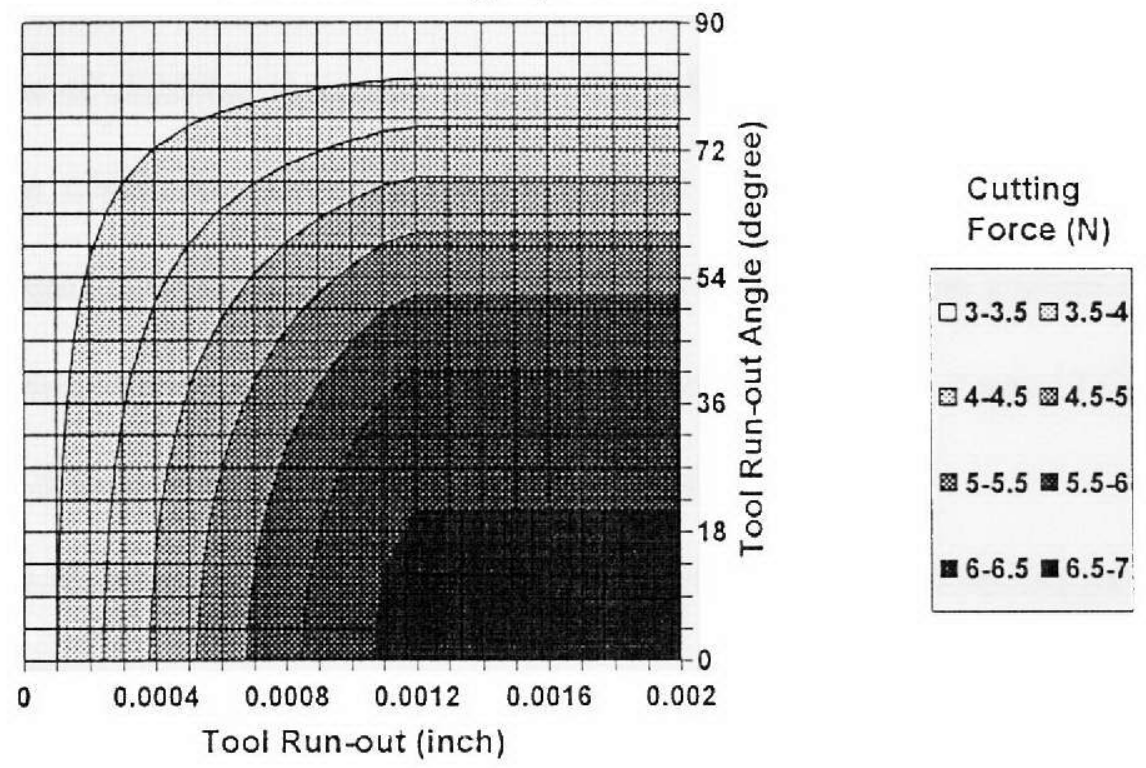

Figure 4.19 2-D cutting force characteristics with tool run-out and its angle

\section{Maximum Cutting Force in Thrust Direction of End Milling Operations}

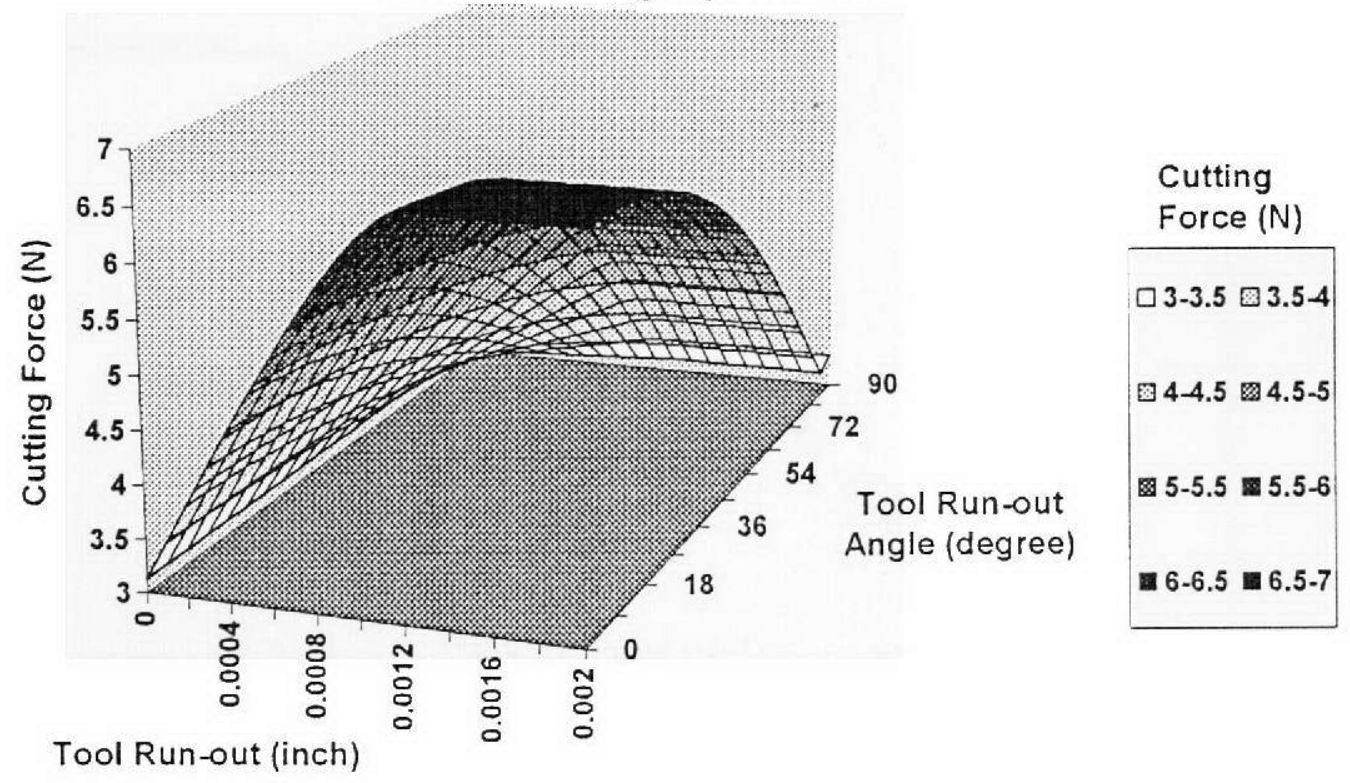

Figure 4.20 3-D cutting force characteristics with tool run-out and its angle 


\section{Cutting Force Characteristics with Tool Cutting Angles}

- Cutting force characteristics with tool entry cutting angle:

The case of climbing micro-end-milling with different percentage overlapping has been studied, in which the exit cutting angle is fixed on 180 degree and entry cutting angles are changed from 0 to 180 degree. The cutting force almost has a linear decrease with the increasing overlapping percentage except the section between 0 and 15 degree, in which it almost is kept in the same level (see Figure 4.21).

The cutting force can be reduced linearly by increasing the overlapping percentage of micro-end-milling.

\section{Maximum Cutting Force in Thrust Direction of End Milling Operations}

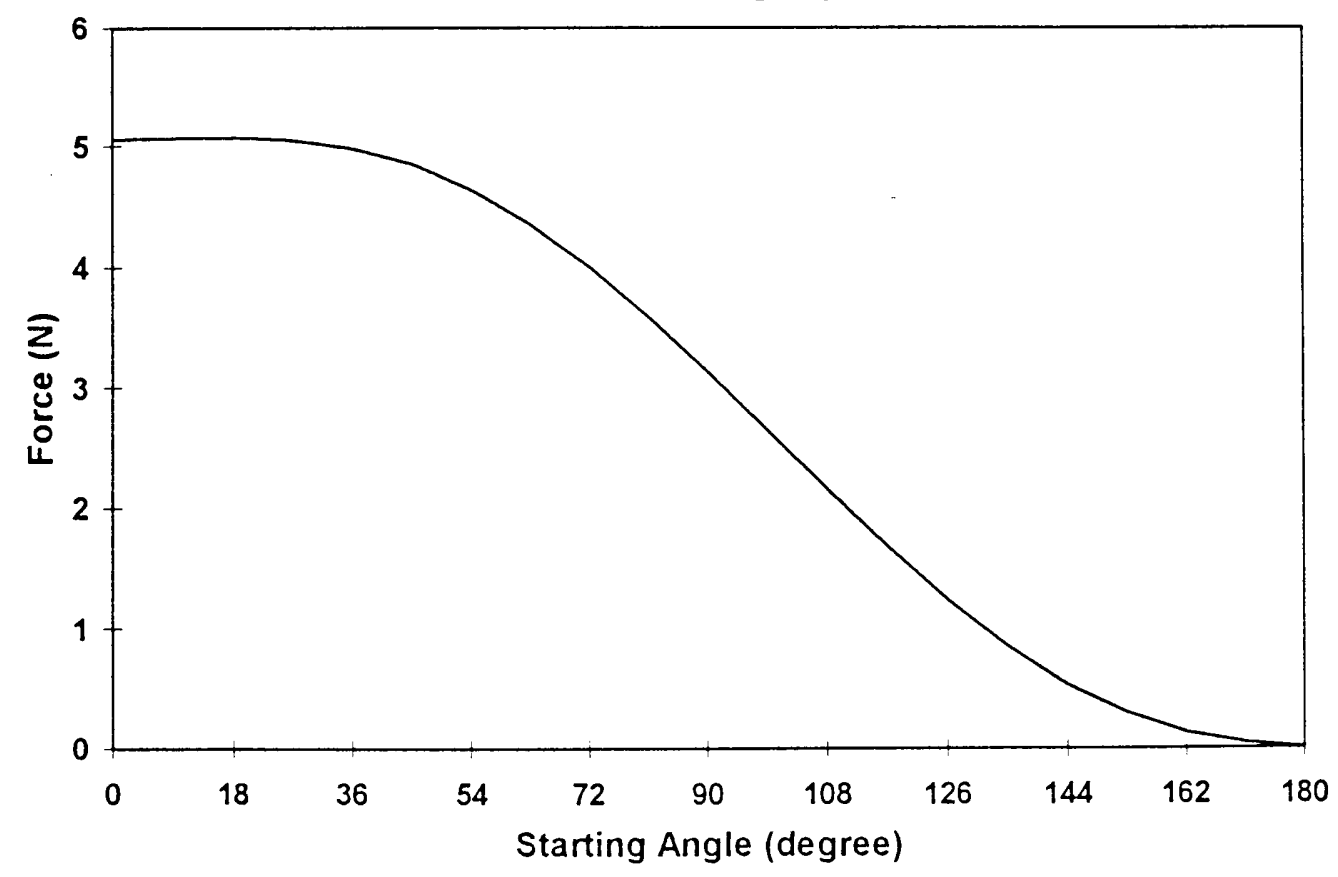

Figure 4.21 Cutting force characteristics with entry cutting angle 
- Cutting force characteristics with tool exit cutting angle:

The case of conventional micro-end-milling with different percentage overlapping has been studied, in which the entry cutting angle is fixed on 0 degree and exit cutting angles are changed from 0 to 180 degree. The cutting force almost has a linear increase with the decreasing overlapping percentage except the section between 0 and 15 degree, in which it almost is 0 (see Figure 4.22).

The same conclusion can be obtained as above that cutting force can be reduced linearly by increasing the overlapping percentage of micro-end-milling.

\section{Maximum Cutting Force in Thrust Direction of End Milling Operations}

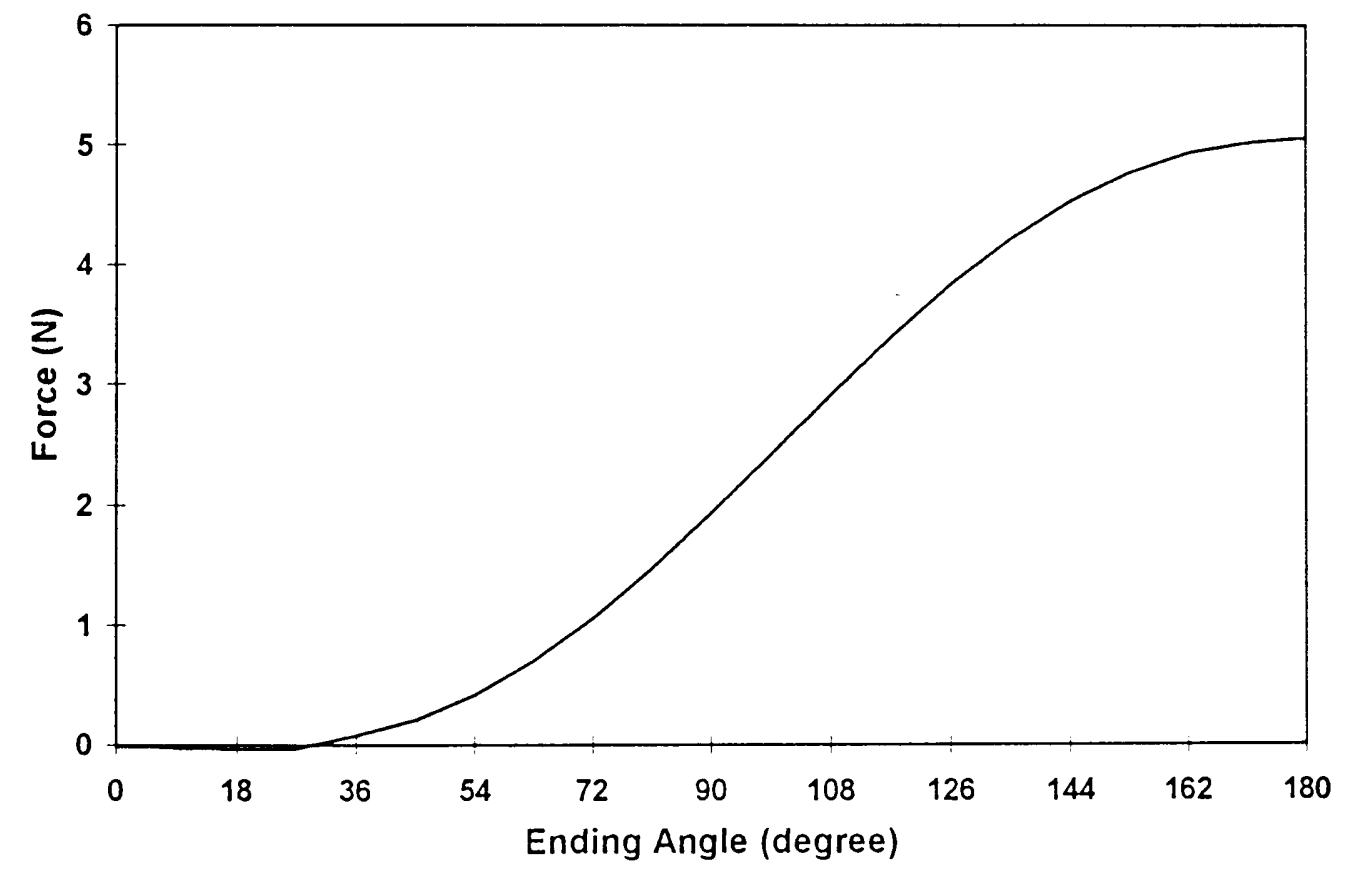

Figure 4.22 Cutting force characteristics with tool exit cutting angle 


\section{Cutting Force Characteristics with Tool Geometry}

- Cutting force characteristics with tool diameter:

The tool diameters from 0.010 " to 0.125 " have been studied. Three different spindle speeds $(15,000,30,000$ and $45,000 \mathrm{rpm})$, feed rates $(20,70$ and $120 \mathrm{ipm})$ and depths of cut (0.010", 0.030 " and $0.050 ")$ are selected in the study.

In all the studied cases, when tool diameter becomes smaller, the cutting force is decreased more rapidly (see Figure 4.23 to 4.25 ). For all the tool diameters, the decrease of the cutting force is almost proportional to the increase of the spindle speed when it is over the critical spindle speed (see Figure 4.23, 4.26 and 4.27).

\section{Maximum Cutting Force in Thrust Direction of End Milling Operations}

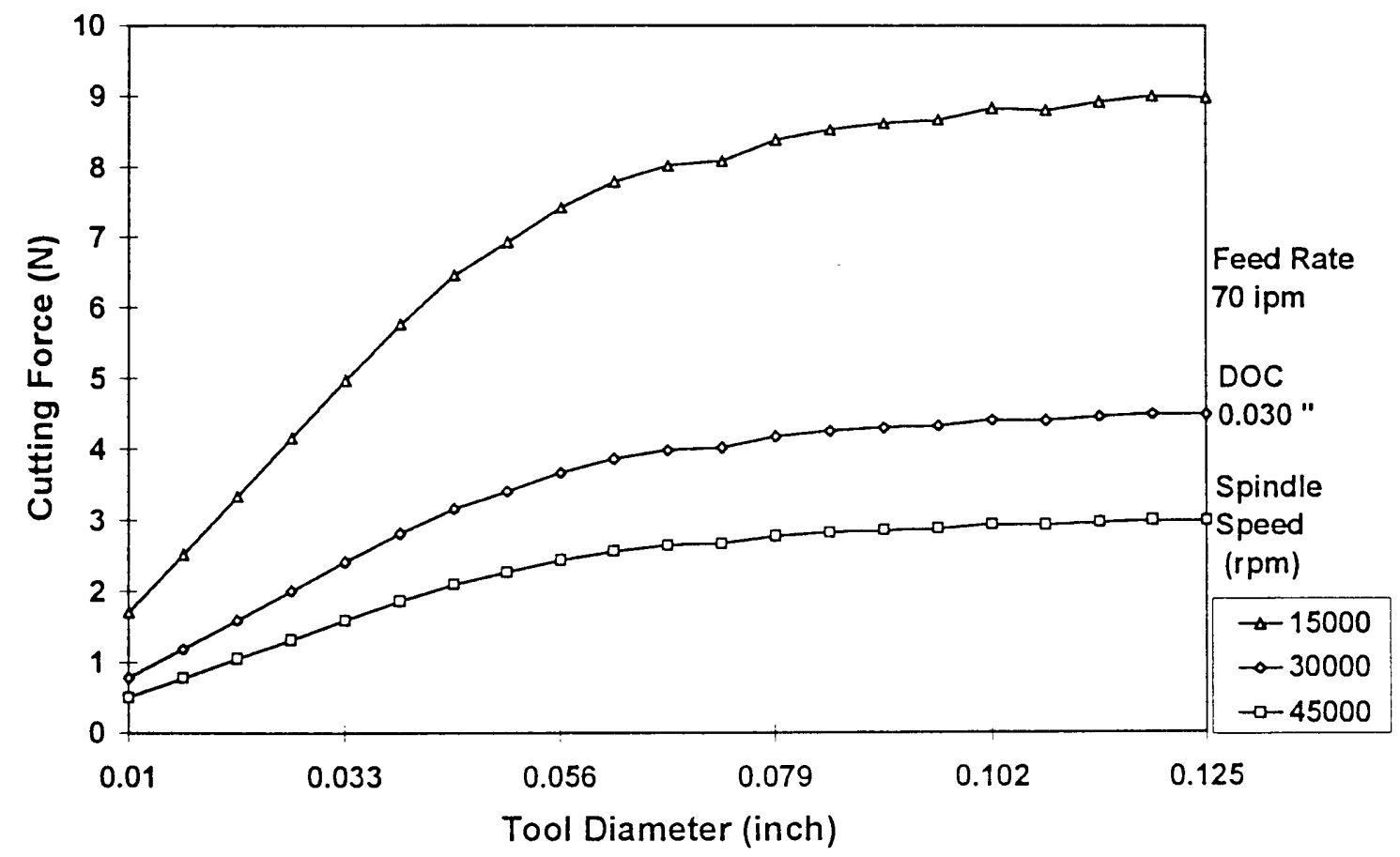

Figure 4.23 Cutting force characteristics with tool diameter and spindle speed 
With different feed rates, the same results can be obtained from the cutting force model. When tool diameter becomes smaller, the cutting force is decreased more rapidly. For all the tool diameters, the decrease of the cutting force becomes almost proportional to the increase of the feed rate (see Figure 4.24, 4.28 and 4.29).

The decreasing slope of the cutting force depends on the tool diameters. The small tool diameter is, the gentler slope of the cutting force becomes.

\section{Maximum Cutting Force in Thrust Direction of End Milling Operations}

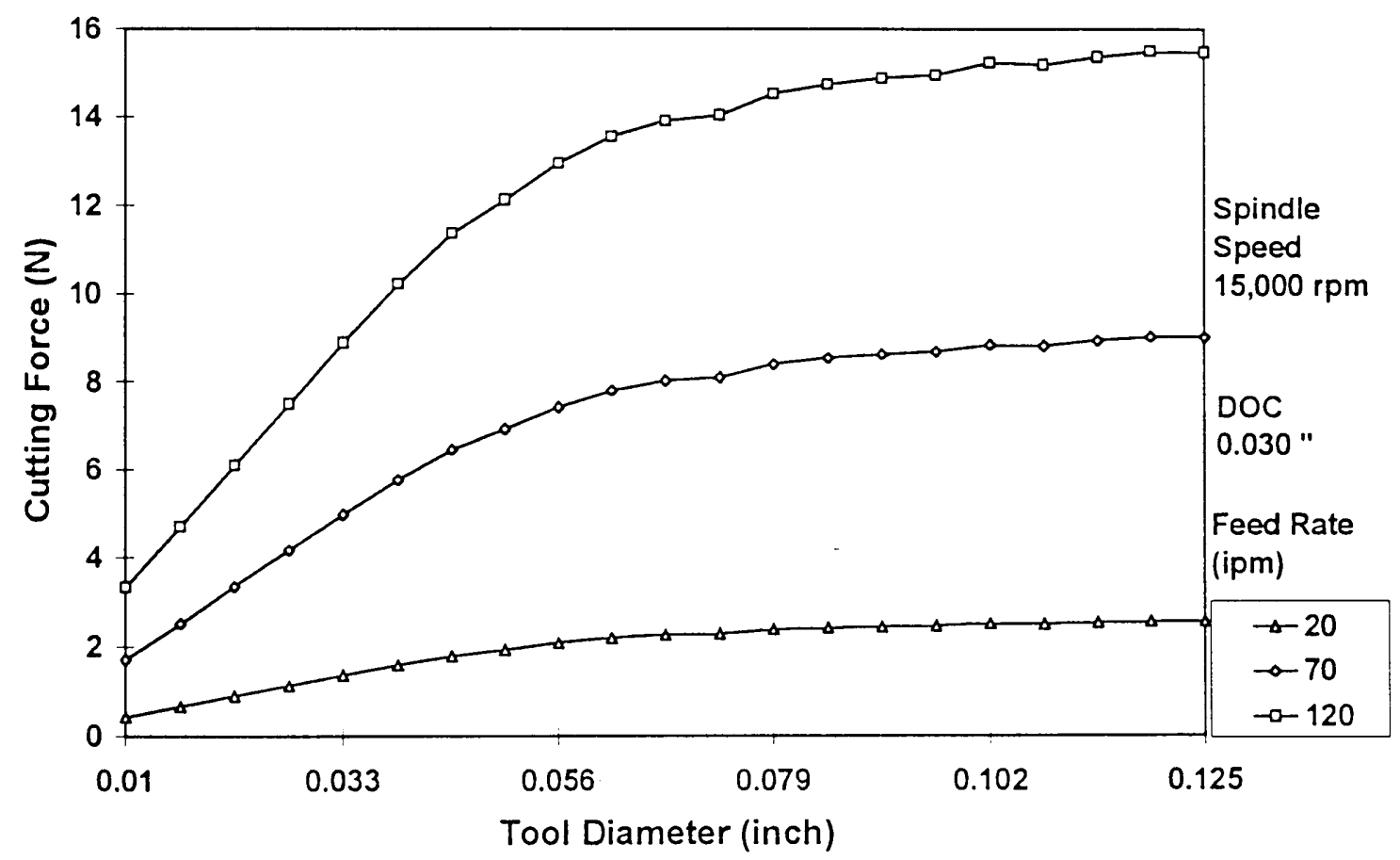

Figure 4.24 Cutting force characteristics with tool diameter and feed rate

For different depths of cut, the same results can be obtained from the analytical cutting force model. When tool diameter becomes smaller, the cutting force is decreased 
more rapidly. For all the tool diameters, the cutting force becomes a constant when the tool diameter is over the critical tool diameter (see Figure 4.25, 4.30 and 4.31).

The critical tool diameter depends on the engagement angle. It can be obtained from the analytical model. In the study case, it can be calculated by following formula.

$$
r_{c}=\frac{b \tan \gamma}{\varphi}
$$

\section{Maximum Cutting Force in Thrust Direction of End Milling Operations}

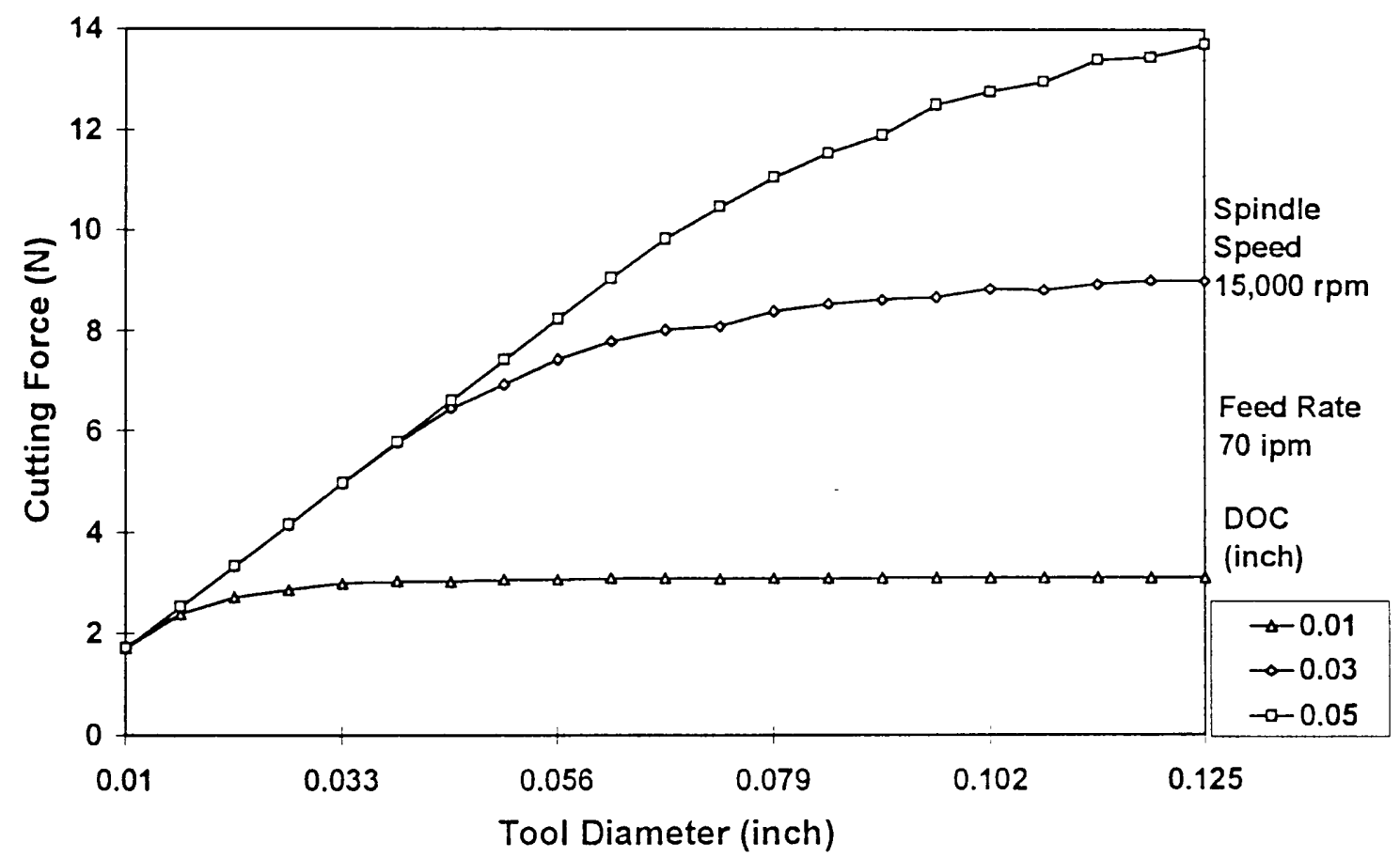

Figure 4.25 Cutting force characteristics with tool diameter and depth of cut

The three dimensional graphics of the cutting force with tool diameter, spindle speed, feed rate and depth of cut are presented in Figure 4.26 to 4.31 . 


\section{Maximum Cutting Force in Thrust Direction of End Milling Operations}

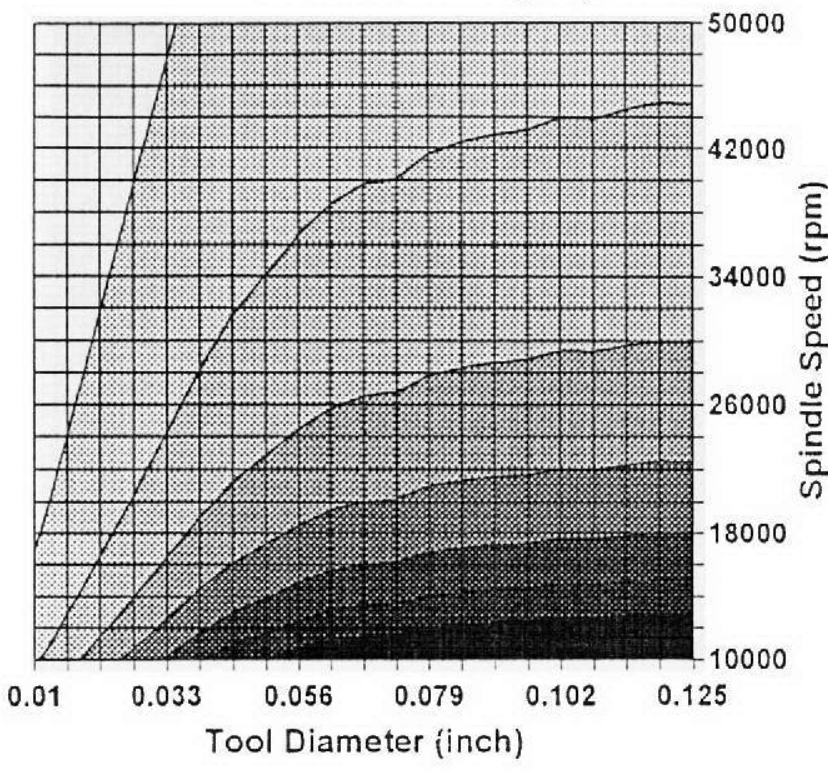

Cutting Force (N)

10.5-12

a $9-10.5$

7.5-9

四-7.5

圆 $4.5-6$

중 $3-4.5$

$\square 1.5-3$

$\square 0-1.5$

Figure 4.26 2-D cutting force characteristics with tool diameter and spindle speed

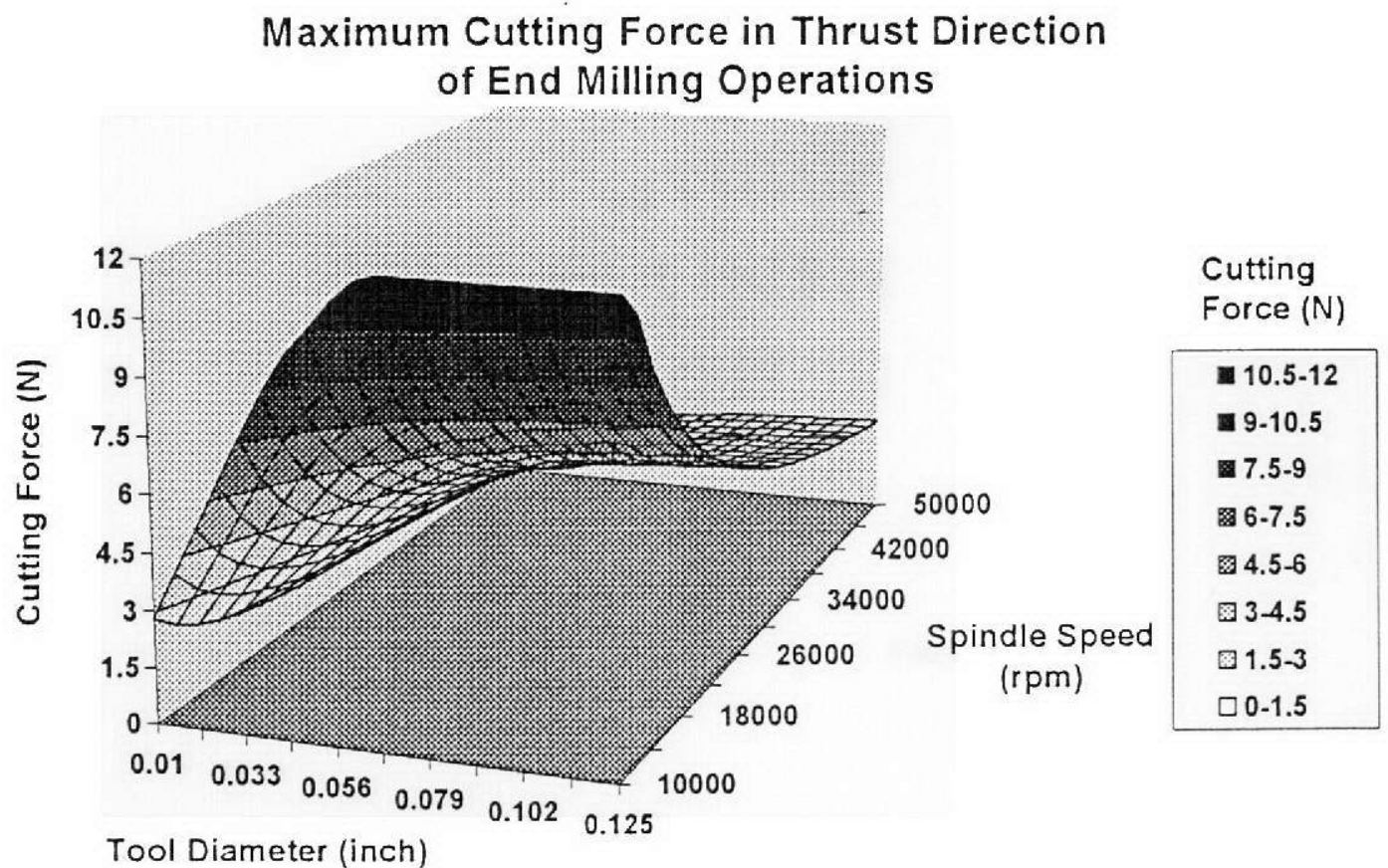

Figure 4.27 3-D cutting force characteristics with tool diameter and spindle speed 


\section{Maximum Cutting Force in Thrust Direction of End Milling Operations}

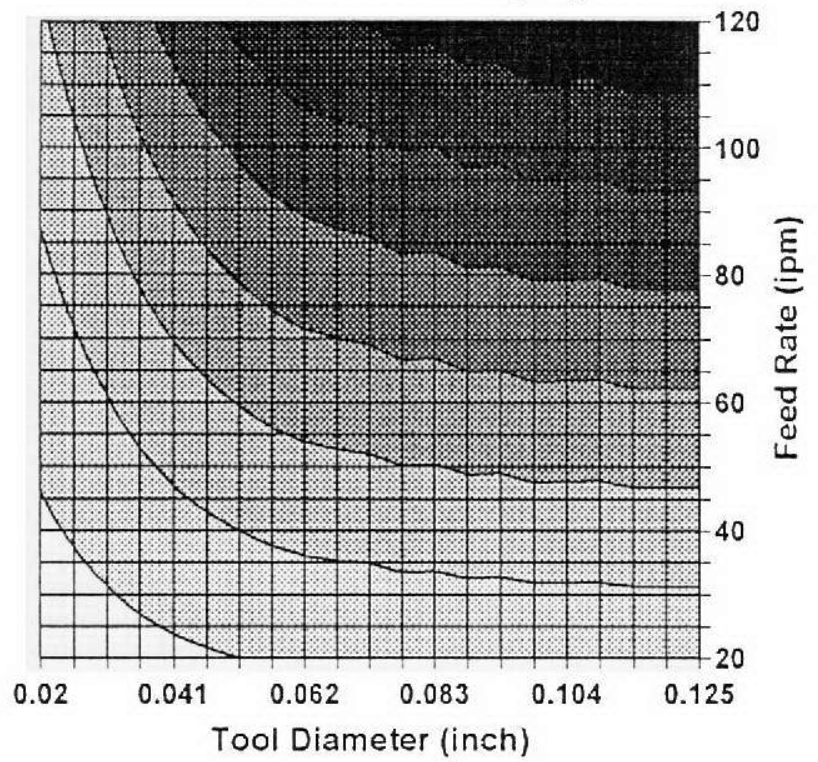

Cutting
Force (N)
\begin{tabular}{l}
$\mathbf{1 4 - 1 6}$ \\
$\square 12-14$ \\
$\square 10-12$ \\
$\square 8-10$ \\
$\square 6-8$ \\
$\square 4-6$ \\
$\square 2-4$ \\
$\square 0-2$ \\
\hline
\end{tabular}

Figure 4.28 2-D cutting force characteristics with tool diameter and feed rate

\section{Maximum Cutting Force in Thrust Direction of End Milling Operations}

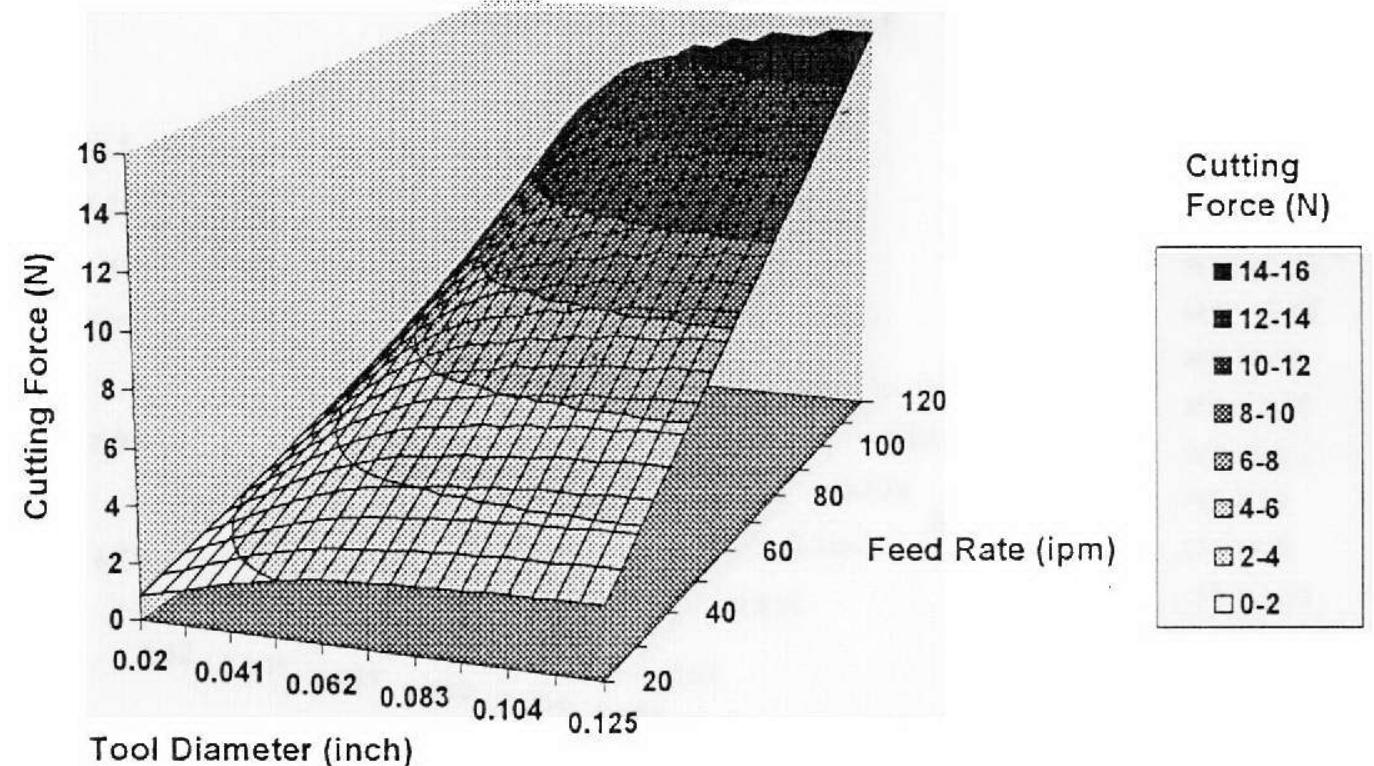

Figure 4.29 3-D cutting force characteristics with tool diameter and feed rate 


\section{Maximum Cutting Force in Thrust Direction of End Milling Operations}

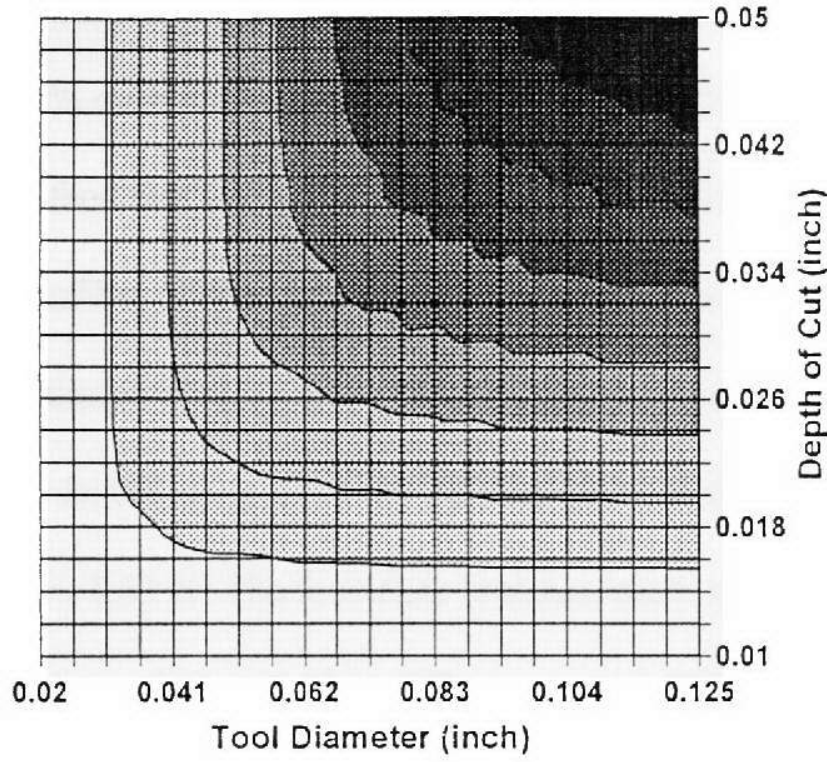

Cutting Force (N)

I $12.25-13.5$

$11-12.25$

훌 9.75-11

요 8.5-9.75

징 7.25-8.5

중 6-7.25

눈 $4.75-6$

$\square 3.5-4.75$

Figure 4.30 2-D cutting force characteristics with tool diameter and depth of cut

\section{Maximum Cutting Force in Thrust Direction of End Milling Operations}

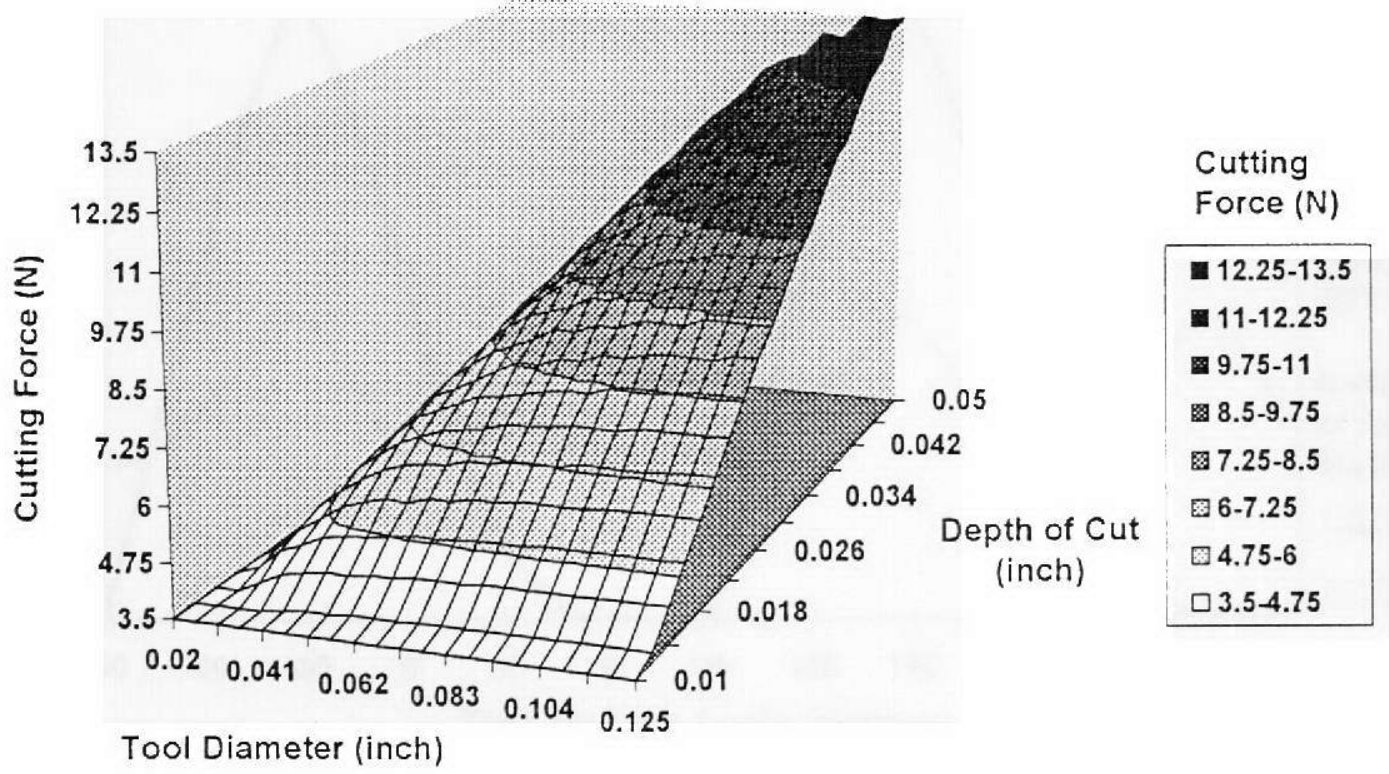

Figure 4.31 3-D cutting force characteristics with tool diameter and depth of cut 
- Cutting force characteristics with tool flute numbers:

Two-flute and four-flute 0.020 " diameter tools have been studied. They are operated in 50\% overlapped climbing operations without tool run-out. 15,000 rpm spindle speed, $70 \mathrm{ipm}$ feed rate and 0.030 " depth of cut are selected in the operations.

The profiles of the cutting force of the thrust direction are presented in Figure 4.32. The maximum cutting force of the four-flute tool is almost the half of one of the two-flute tool. In the study case, the cutting force of the four-flute tool is $1.30 \mathrm{~N}$ and the two-flute tool is $2.63 \mathrm{~N}$. The four-flute tool has much smaller cutting force oscillation than the two-flute tool, which is $0.65 \mathrm{~N}$ difference and $2.63 \mathrm{~N}$ difference, or $1: 4$.

\section{Cutting Force in Thrust Direction of End Milling Operations}

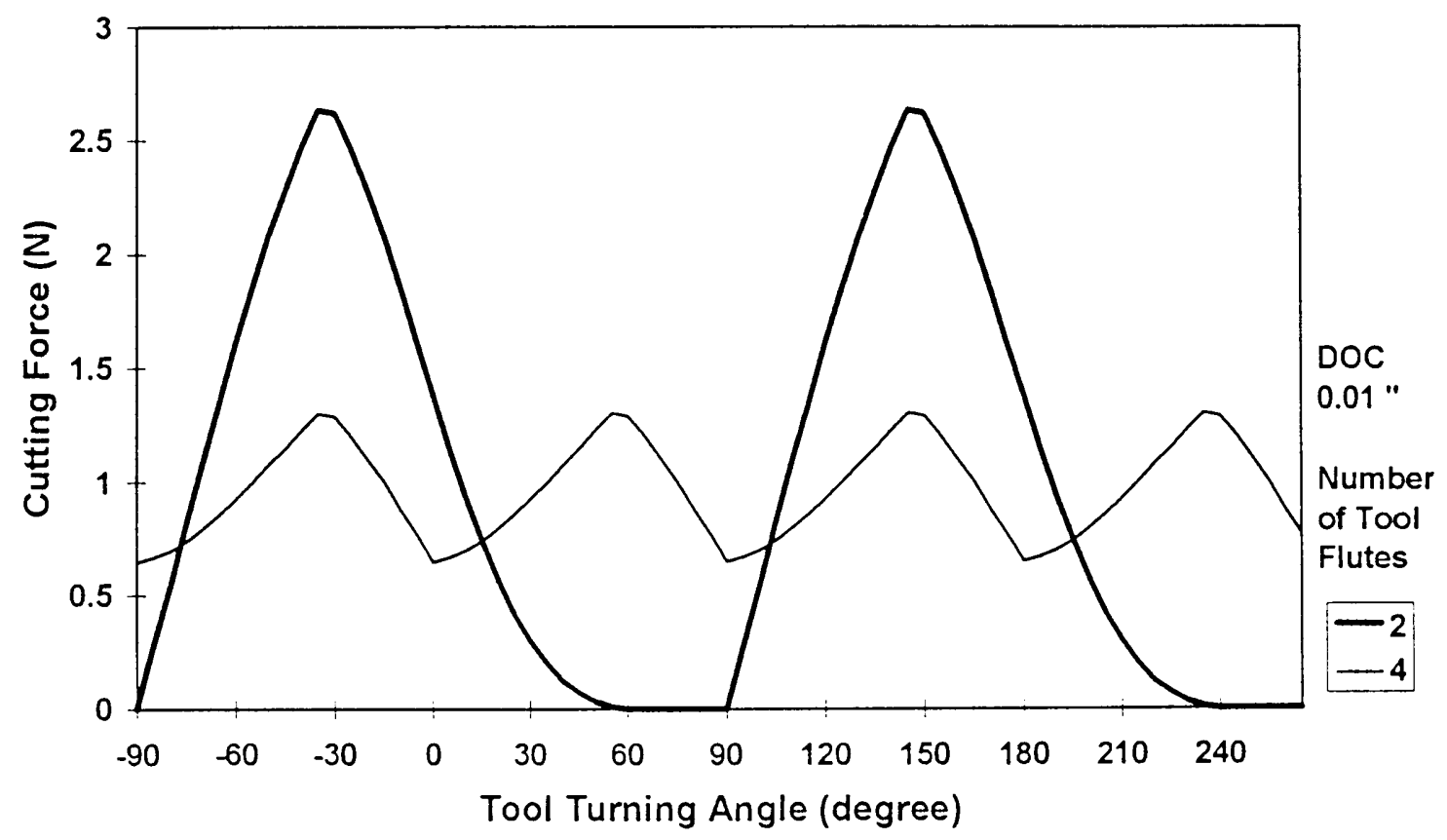

Figure 4.32 Cutting force characteristics with toot flute numbers 
- Cutting force characteristics with tool helix angle:

The tool helix angles from 25 to 75 degrees are studied. The cutting force almost has a linear decrease when tool helix angle is increased (see Figure 4.33).

\section{Maximum Cutting Force in Thrust Direction of End Milling Operations}

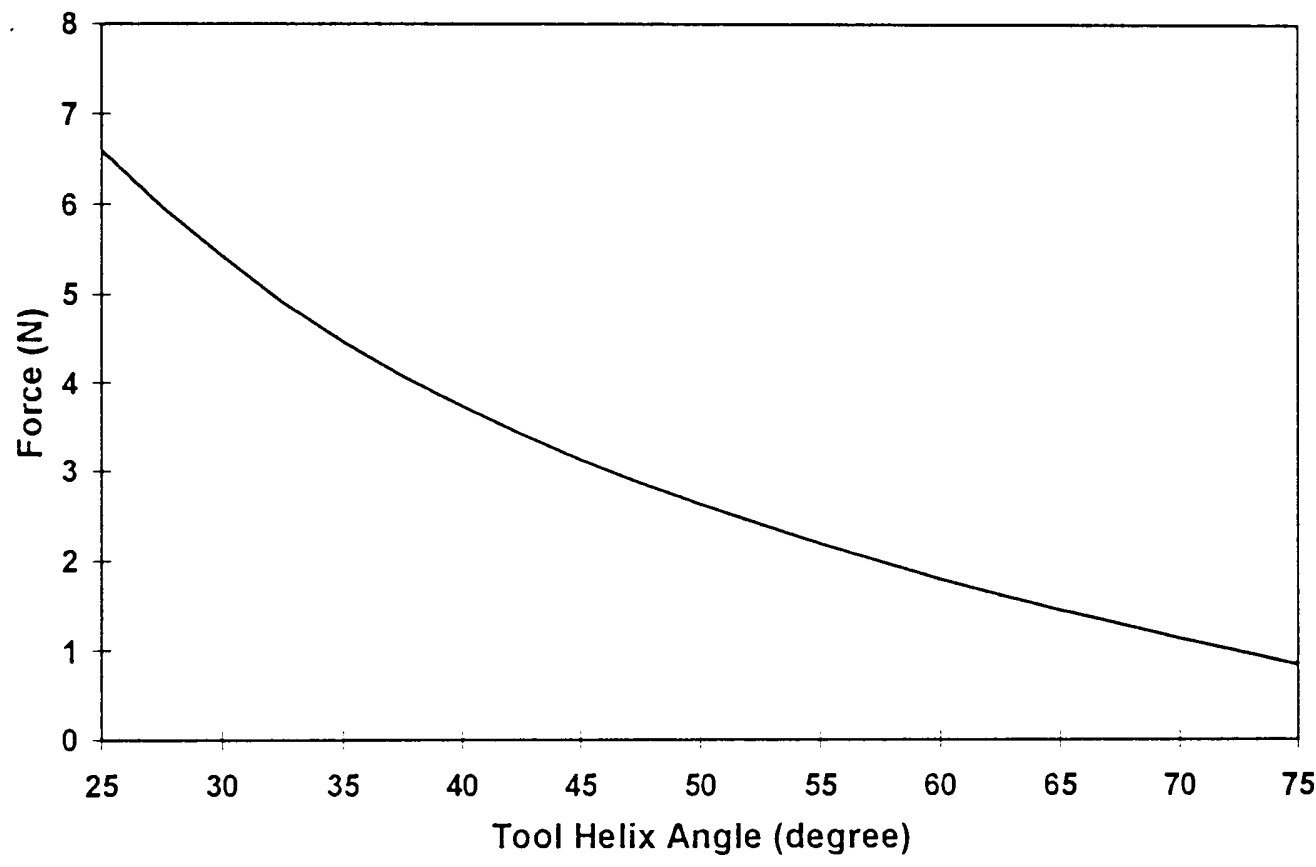

Figure 4.33 Cutting force characteristics with toot helix angle 


\subsection{Work-piece Surface Roughness and Precision}

\section{Work-piece Surface Roughness}

It is important for the machining operations to obtain the request surface quality of the work-piece. Work-piece surface roughness is one of the surface quality indicant. It can be derived from the developed analytical cutting force model by considering the tool cutting edge tip profiles (see Figure 4.34).

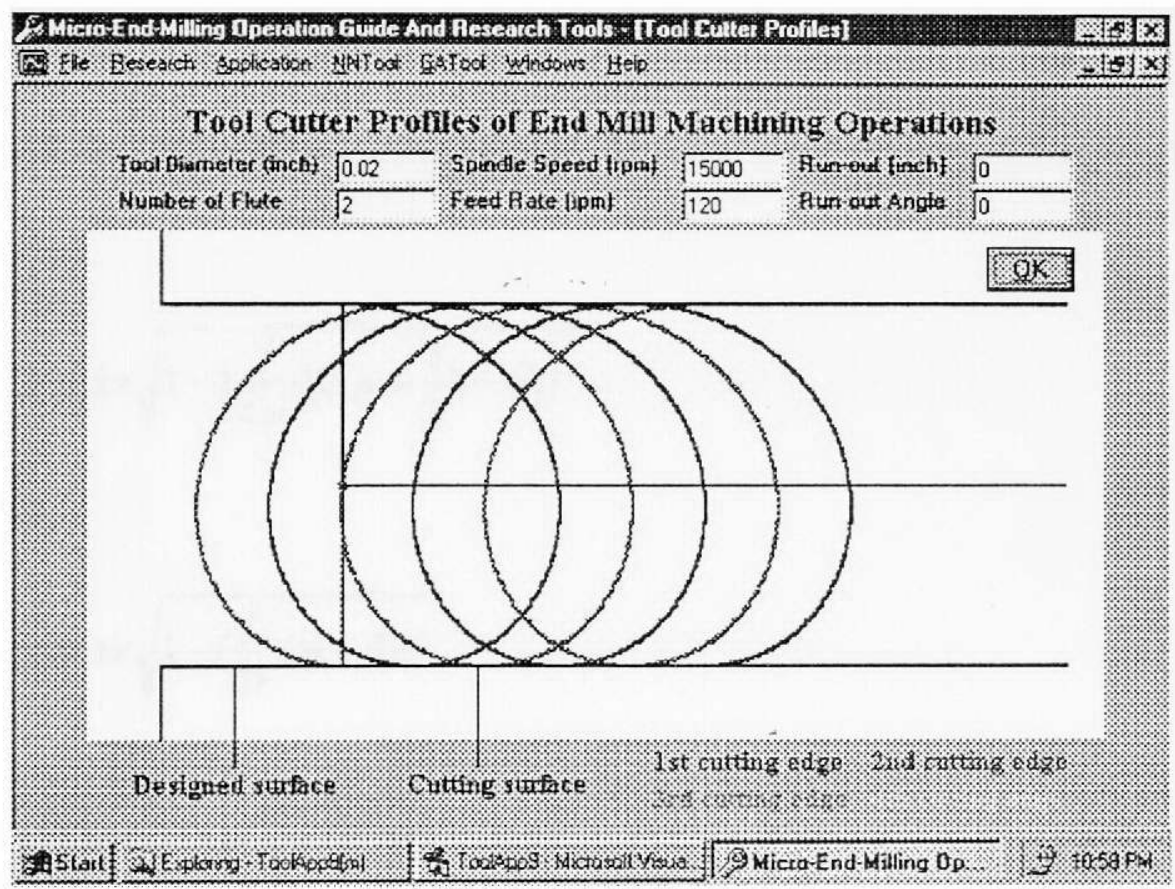

Figure 4.34 Work-piece surface roughness by considering the tool cutting edge tip profiles

Substituting $\mathrm{t}$ from the equations 3.1 .1 to 3.1 .2 , the equations of the tool cutting edge tip profiles become:

$$
\left\{x-\frac{f}{2 n \pi}\left[\arccos \left(\frac{y}{r}\right)+\frac{2 z \pi}{Z}\right]\right\}^{2}+y^{2}=r^{2}
$$

where: $z=0,1$ for two-flute tools. 


$$
z=0,1,2,3 \text { for four-flute tools. }
$$

Two equations can be derived from equation 4.3 .1 by considering the $1^{\text {st }}$ path of the first flute $(z=0)$ and the $p^{\text {th }}$ path of any flutes (included the first flute itself).

$$
\begin{aligned}
& \left\{x-\frac{f}{2 n \pi}\left[\operatorname{Arccos}\left(\frac{y}{r}\right)\right]\right\}^{2}+y^{2}=r^{2} \\
& \left\{x-\frac{f}{2 n \pi}\left[2\left(p+\frac{z}{Z}\right) \pi-\operatorname{Arccos}\left(\frac{y}{r}\right)\right]\right\}^{2}+y^{2}=r^{2}
\end{aligned}
$$

where: $p=0,1,2,3, \ldots$

All the roots of the cutting edge profile can be solved from the equations 4.3 .2 and

\subsection{3.}

$y= \pm r \sqrt{1-\left\{\frac{f}{4 n r}\left[2\left(p+\frac{z}{Z}\right)-\delta\right]\right\}^{2}}$

or:

$y= \pm r \sqrt{1-\left[\frac{f_{t}}{2 r}(m-\delta)\right]^{2}}$

where: $\delta=\frac{2}{\pi} \operatorname{Arccos}\left(\frac{y}{r}\right)$

$$
\mathrm{m}=1,2,3, \ldots \text { until } \mathrm{m} \leq 4 \mathrm{nr} / \mathrm{f} \text {. }
$$

Because only working surface profiles are interested, $y$ value is very close to $r$ value. That $\delta=0$ is assumed. The formula becomes:

$$
y= \pm r \sqrt{1-\left(\frac{m f_{t}}{2 r}\right)^{2}}
$$

The work-piece surface roughness is: 
$S_{r}=r-|y|=r\left[1-\sqrt{1-\left(\frac{m f_{t}}{2 r}\right)^{2}}\right]$

Considering that $\mathrm{f}_{\mathrm{l}} /(2 \mathrm{r})$ is small, the formula can be approximated as:

$$
S_{r} \approx \frac{\left(m f_{t}\right)^{2}}{4 d}
$$

When $\mathrm{m}=1$, the formula 4.3 .6 gives an approximate value for both conventional and climbing milling. Actually, the work-piece surface roughness is a little smaller than that approximate value in conventional milling and a little larger in climbing milling. Conservatively, $\mathrm{m}=1$ can be used for conventional milling and $\mathrm{m}=\sqrt{2}$ for climbing milling.

For conventional milling, the work-piece surface roughness is:

$$
S_{r} \approx \frac{f_{t}^{2}}{4 d}
$$

For climbing milling, the work-piece surface roughness is:

$$
S_{r} \approx \frac{f_{t}^{2}}{2 d}
$$

The results have a similar form to the formula derived by Martellotti, mentioned in Milton C. Shaw's "Metal Cutting principles" ${ }^{[43]}$.

If request surface roughness is $S_{r}$, the working conditions have to be selected as following formulas.

For conventional milling:

$$
f_{t} \leq 2 \sqrt{S_{r} d}
$$

For climbing milling: 


$$
f_{t} \leq \sqrt{2 S_{r} d}
$$

The work-piece surface roughness is proportional to the square of the feed per tooth and inverse proportional to the diameter of the tool. The faster spindle speed and the slower feed rate, the better quality work-piece surfaces would be obtained. In theory, the work-piece surfaces could be manufactured to an absolutely smooth plane when a tiny feed rate and fast enough spindle speed were given.

\section{Work-piece Surface Precision}

Work-piece surface precision is anther important surface quality indicant. It can be derived from the analytical cutting force model by considering the tool run-out which is the most important factor of the work-piece geometrical accuracy (see Figure 4.35)

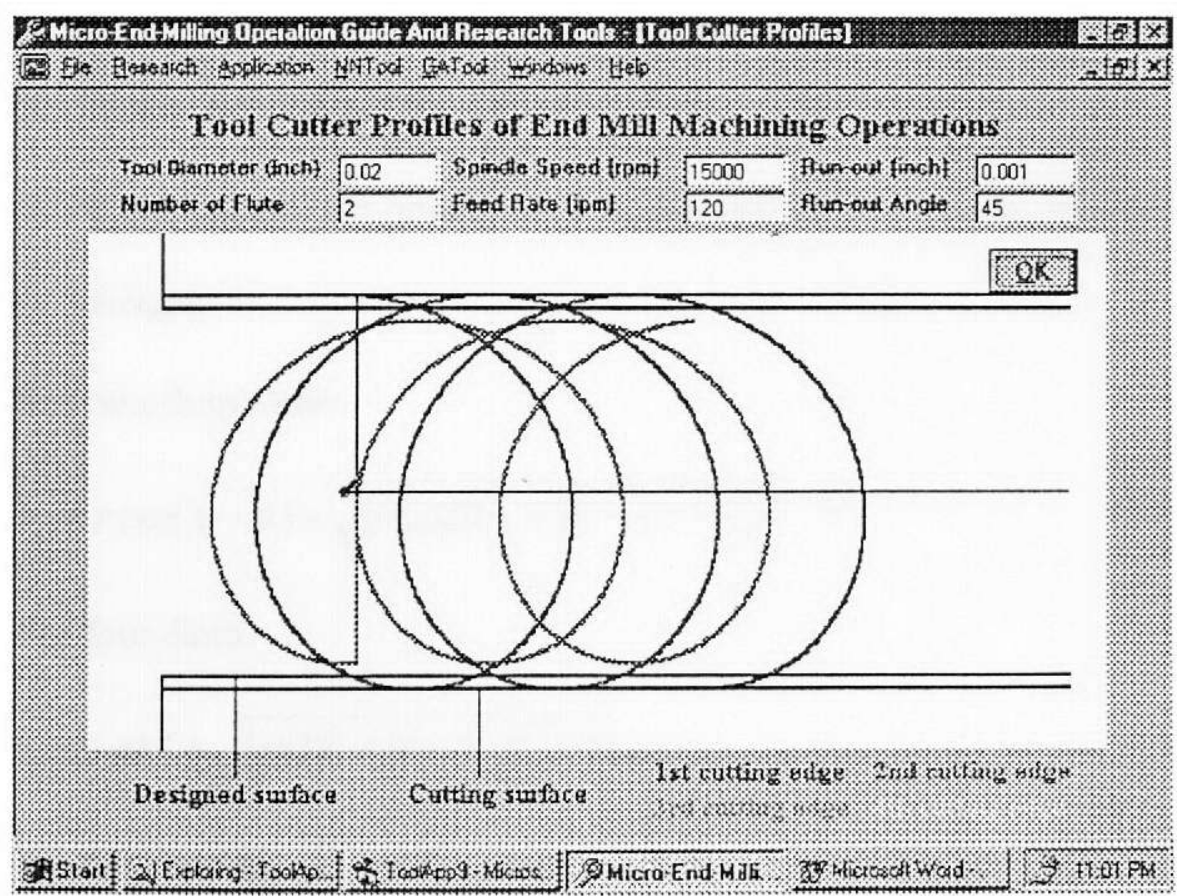

Figure 4.35 Work-piece surface precision by considering the tool run-out 
Considering the tool run-out cutting edge tip profile equations 3.2 .1 and 3.2 .2 , and the geometry of the tool run-out, the work-piece surface precision can be derived.

For two-flute tools, the work-piece surface precision is:

$$
S_{p}=\sqrt{r^{2}+r_{o}^{2}-2 r r_{o} \cos (\pi-\gamma)}-r
$$

For four-flute tools, the work-piece surface precision is:

$$
S_{p}=\sqrt{r^{2}+r_{o}^{2}-2 r r_{o} M}-r
$$

where: $M=\operatorname{Max}\{\operatorname{Abs}[\cos (\pi-\gamma)], \operatorname{Abs}[\cos (\pi / 2+\gamma)]\}$

When the tool run-out angle is parallel to the tool cutting edges $(\gamma=0)$, the workpiece surface has the lowest precision. When the tool run-out angle is perpendicular to the tool cutting edges $(\gamma=\pi / 2)$ for two-flute tools or 45 degree to the two adjacent tool cutting edges $(\gamma=\pi / 4)$ for four-flute tools, the work-piece surface has the highest precision.

If the request surface roughness is $S_{p}$, the tool run-out has to be considered as the following formulas.

For two-flute tools:

$$
r_{o} \leq r \cos (\pi-\gamma)+\sqrt{[r \cos (\pi-\gamma)]^{2}+\left(r+S_{p}\right)^{2}-r^{2}}
$$

For four-flute:

$$
r_{o} \leq-r M+\sqrt{(r M)^{2}+\left(r+S_{p}\right)^{2}-r^{2}}
$$

Usually, the run-out angle can be considered as $\pi / 2$ for two-flute tools or $\pi / 4$ for four-flute tools. Conservatively, the run-out angle can be considered as 0 . 


\section{Chapter V}

\section{Model Based Monitoring of Micro-End-Milling Operations}

Genetic algorithm based optimization method is proposed for the monitoring of micro-end-milling operations. It is possible to monitor tool wear, breakage, run-out, cutting angles and cutting conditions by using this approach. The sum of the difference of cutting forces obtained from the monitoring signals and the analytical cutting force model is considered as the objective function. It is optimized by a genetic algorithm program (GATool) to search the fittest variables of the analytical cutting force model. It has been proved that information included in the cutting force signals can be identified by the proposed method. The tool wear, run-out, cutting angles and cutting conditions of microend-milling operations can be estimated in a few evolution generations with an acceptable error. The GATool program has a fast reaction and accurate results that can be applied to the on-line monitoring of micro-end-milling operations. The performance of the genetic algorithm based monitoring method is presented in this chapter.

\subsection{Tool Breakage Detection}

In micro-end-milling operations, it is hard to see or heard when the tool is broken because of its tiny size. Tool breakage monitoring methods can avoid wasting machining time, tools and work-pieces. It can stop the machining operation immediately and warn the operator to change the tool or to adjust the tool working conditions to extend the tool life until the task is completed. 
The tool breakage could be caused by many different reasons that included unsuitable working conditions, dulled and damaged tool cutting edges, tool cutting edges stuck with the milted chips and so forth. To detect the tool breakage and to understand its reason, two most commonly used signals are acoustic emission (AE) and cutting force. In this study, acoustic emission (AE) is used to monitor the tool breakage, and the cutting forces are used to explain the reason of the tool breakage.

\section{Detecting tool breakage by monitoring machining acoustic emission ${ }^{[27-30]}$}

Acoustic emission signal characteristics change with the tool conditions. The signal characteristics are different when the operation and tool (wear, breakage) conditions are changed. In this section, a new method is proposed to detect tool breakage without giving false alarm when the tool leave the work-piece.

In the experiments, the $\mathrm{AE}$ sensor was attached on the work-piece directly. For analog signal processing, the DME Corporation's SWAN 3000 system was used. The AE signal was filtered at $40 \mathrm{KHz}$ modulated and sampled by a Nicolet 310 digital oscilloscope.

Two-flute high speed steel end mill with 0.015 " diameter was used to machine mild steel work-piece. The cutting conditions were $30,000 \mathrm{rpm}$ spindle speed, $0.24 \mathrm{ipm}$ feed rate and 0.016 " depth of cut in the experiment $\mathrm{I}$, and 3,000 rpm spindle speed, 0.9 ipm feed rate and 0.005 " depth of cut in the experiment II. The AE signals at the both experiments are presented in Figure 5.1 to 5.4.

To distinguish the tool breakage from leaving the work-piece, two algorithms are developed. 
ACOUSTIC EMISSION TESTING IN END-MILL

MACHINING OPERATIONS

(experiment I: tool broken case)

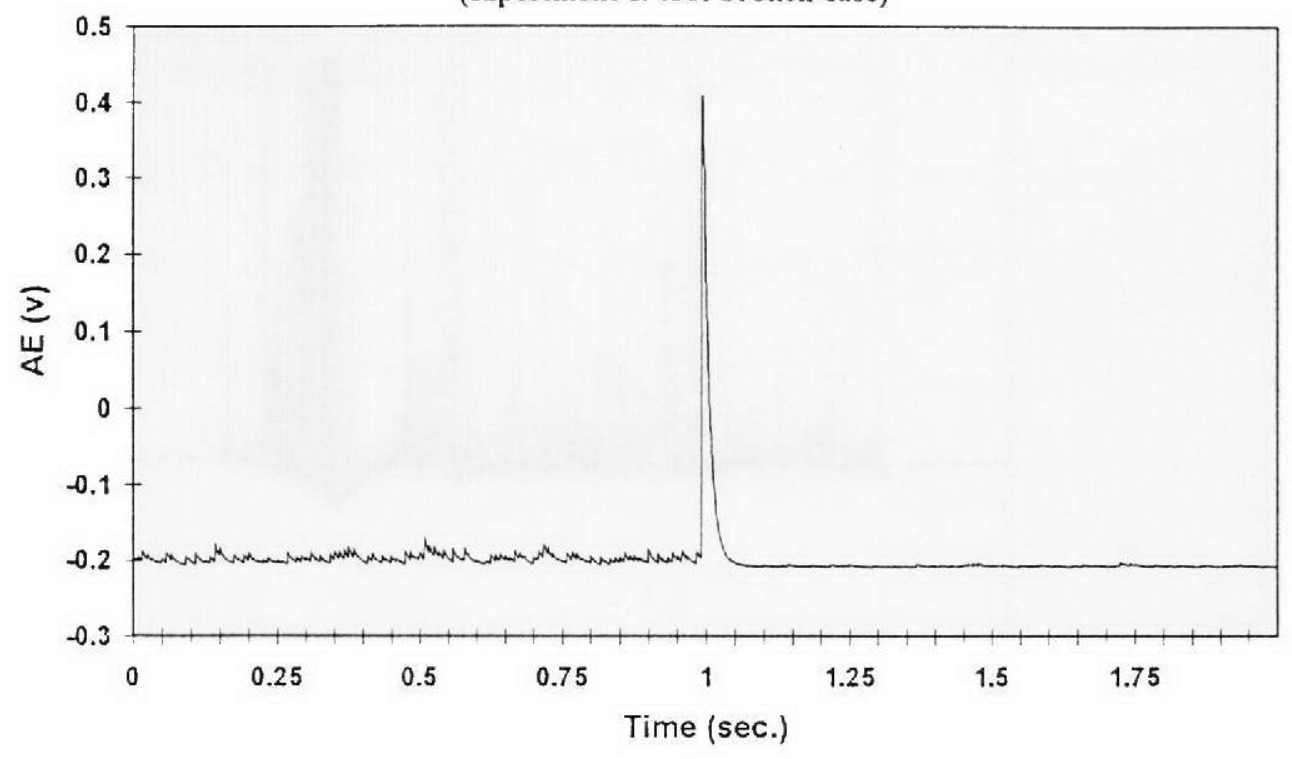

Figure 5.1 AE activity of micro-end-milling experiment $\mathrm{I}$, case 1

\section{ACOUSTIC EMISSION TESTING IN END-MILL}

MACHINING OPERATIONS

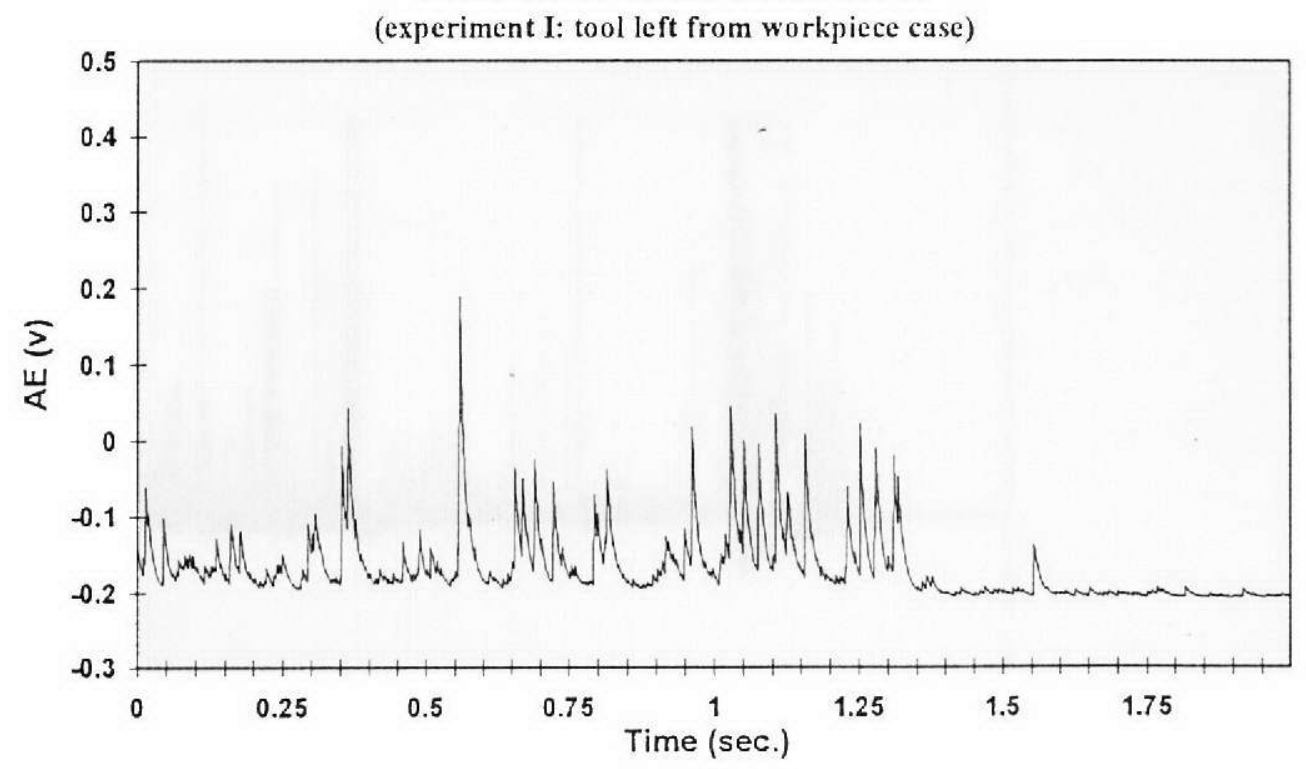

Figure 5.2 AE activity of micro-end-milling experiment I, case 2 
ACOUSTIC EMISSION TESTING IN END-MILL

MACHINING OPERATIONS

(tool broken case 1)

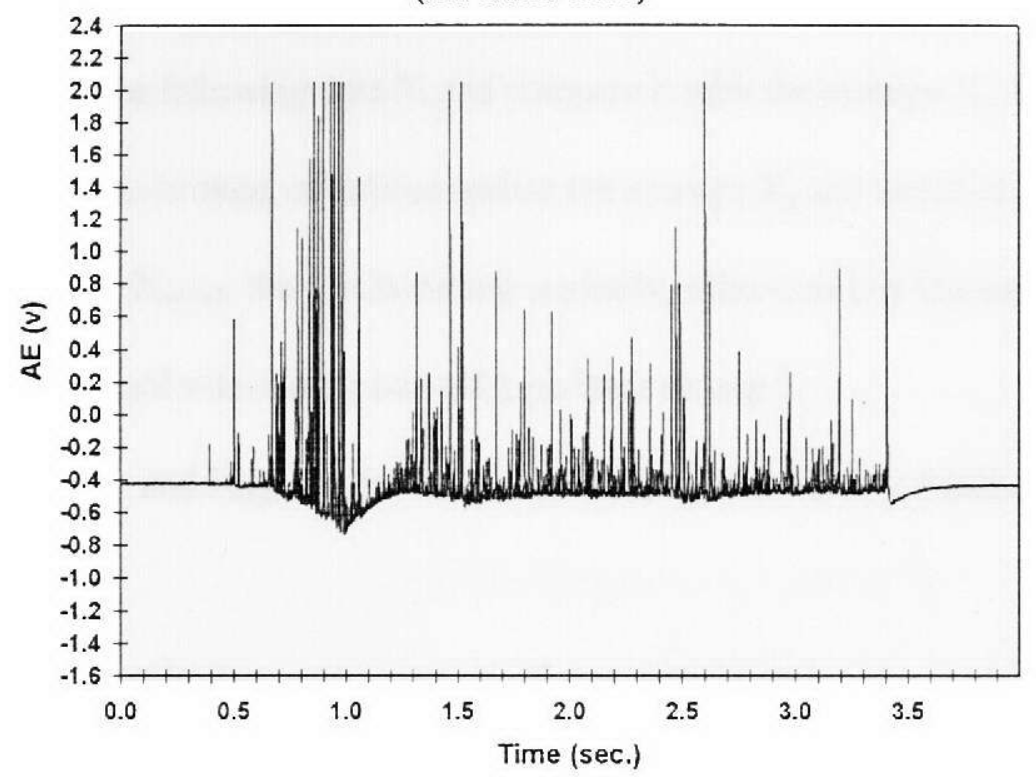

Figure 5.3 AE activity of micro-end-milling experiment II, case 1

ACOUSTIC EMISSION TESTING IN END-MILL MACHINING OPERATIONS

(tool left from workpiece case 1)

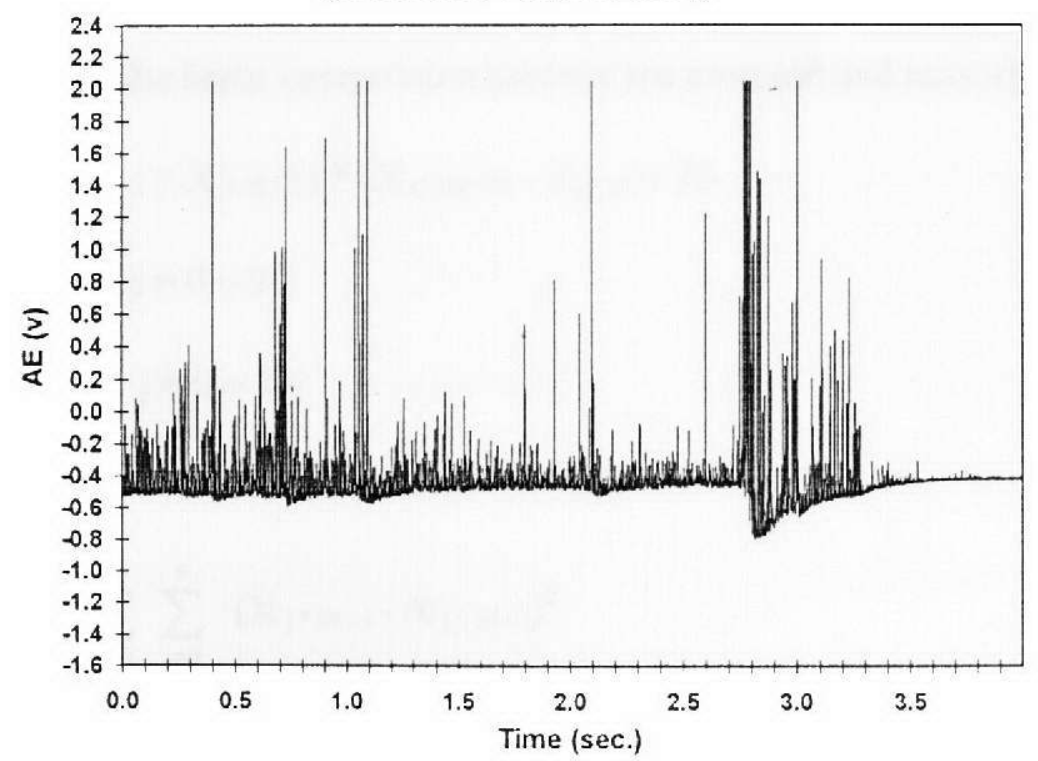

Figure 5.4 AE activity of micro-end-milling experiment II, case 2 
Method 1: Tool broken with a very sharp spike signal

Step 1. Calculate the average $X_{a}$ and variation $X_{v}$ of the first 150 data.

Step 2. Take the following data $X_{i}$ and compare it with the average $X_{a}$. If $X_{i}>X_{a}+X_{\text {high, }}$, the tool was broken, otherwise update the average $X_{a}$ and variation $X_{v}$ by considering $X_{i}$.

Step 3. If $\mathrm{X}_{\mathrm{v}}>\mathrm{X}_{\mathrm{momal}}$, the tool is cutting normally, otherwise it is leaving from the work-piece. If the tool was cutting normally, go back to step 2 .

$\mathrm{X}_{\text {nomal }}$ and $\mathrm{X}_{\text {high }}$ can be decided by studying the $\mathrm{AE}$ data pattern.

Method 2: Tool broken with a non-significant spike signal

Step 1. Calculate the average $\mathrm{X}_{\mathrm{a}}$ of the first 150 data.

Step 2. Take the following data $X_{i}$ and compare it with the average $X_{a}$. If $X_{i}<X_{a}-X_{\text {low }}$, go to step 3, otherwise update the average $X_{a}$ by considering $X_{i}$ and then repeat step 2 .

Step 3. Take the following 150 data. Create 15 sections with 10 data each. For each section $j$ obtain the linear interpolation between the most-left and most-right points: $X_{j * 10+i}^{\prime}=X_{j * 10}+i *\left(X_{(j+1) * 10}-X_{j * 10}\right) / 10$ where: $\mathrm{i}=0 \sim 9$

$$
j=0 \sim 14
$$

Step 4. Estimate the total error of the 150 data

$$
\mathrm{E}=\sum_{j=0}^{14} \sum_{i=0}^{9}\left(\mathrm{X}_{\mathrm{j} * 10+\mathrm{i}}-\mathrm{X}_{\mathrm{j} * 10+\mathrm{i}}^{\prime}\right)^{2}
$$

Step 5. Compare the error with two reference values $E_{\text {normal }}$ and $E_{\text {breakage, }}$ which can be decided by studying the $\mathrm{AE}$ data patterns. Make the decision by the following rules: 


$$
\begin{array}{ll}
\mathrm{E}<\mathrm{E}_{\text {normal }} & \text { The tool is cutting normally. } \\
\mathrm{E}_{\text {nomal }} \geq \mathrm{E} \geq \mathrm{E}_{\text {breakage }} & \text { The tool is leaving the work-piece. } \\
\mathrm{E}_{\text {breakage }}<\mathrm{E} & \text { The tool was broken. }
\end{array}
$$

If the tool was cutting normally, go back to step 2 . In case the tool is broken or leaving the work-piece, stop the process.

Both the algorithms detected tool breakage in all the tests without any error. The results will be discussed in Chapter VII (Figure 7.3.1 and 7.3.2).

The AE based tool breakage detection methods can be easily implemented and require relatively simple instrumentation. They are most of the time reliable, however, if unexpected noises are created in the system because of friction they may fail.

\section{Detecting tool breakage by monitoring cutting force ${ }^{[31]}$}

The characteristics of the cutting force change with tool wear. When the cutting force increase beyond a critical value, miniature tools break. The objective of the method in this section is to identify the tool breakage.

In the experiments, the work-piece was installed on a Kistler 9257B dynamometer that was attached to the table. Two-dimensional cutting force signals were collected by a Nicolet 310 digital oscilloscope through a Kistler three channel charge amplifier. The experiment setup is presented in Chapter VI.

Two-flute high speed steel end mill with 0.015 " diameter was used to machine mild steel and aluminum work-pieces. The working conditions were $30,000 \mathrm{rpm}$ spindle 
Tool Cutting Force of End-mill Machining Operations (complete tool life cycle, mild steel workpiece case)

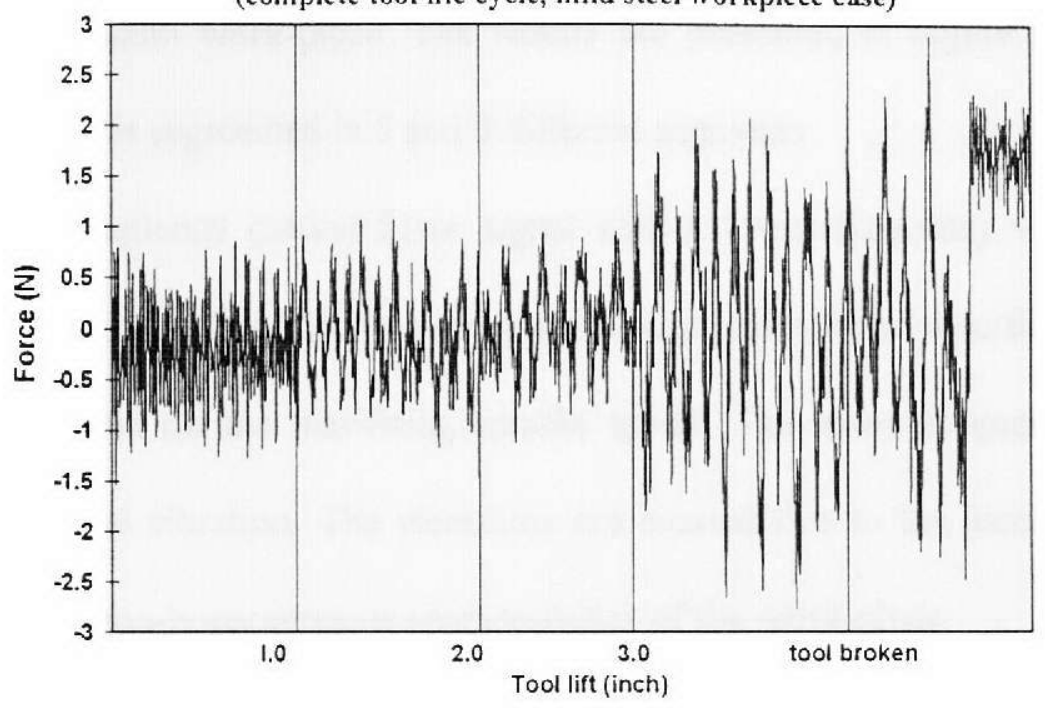

Figure 5.5 Tool cutting force of micro-end-milling experiment I

Tool Cutting Force of End-mill Machining Operations (complete tool life cycle, aluminum workpiece case)

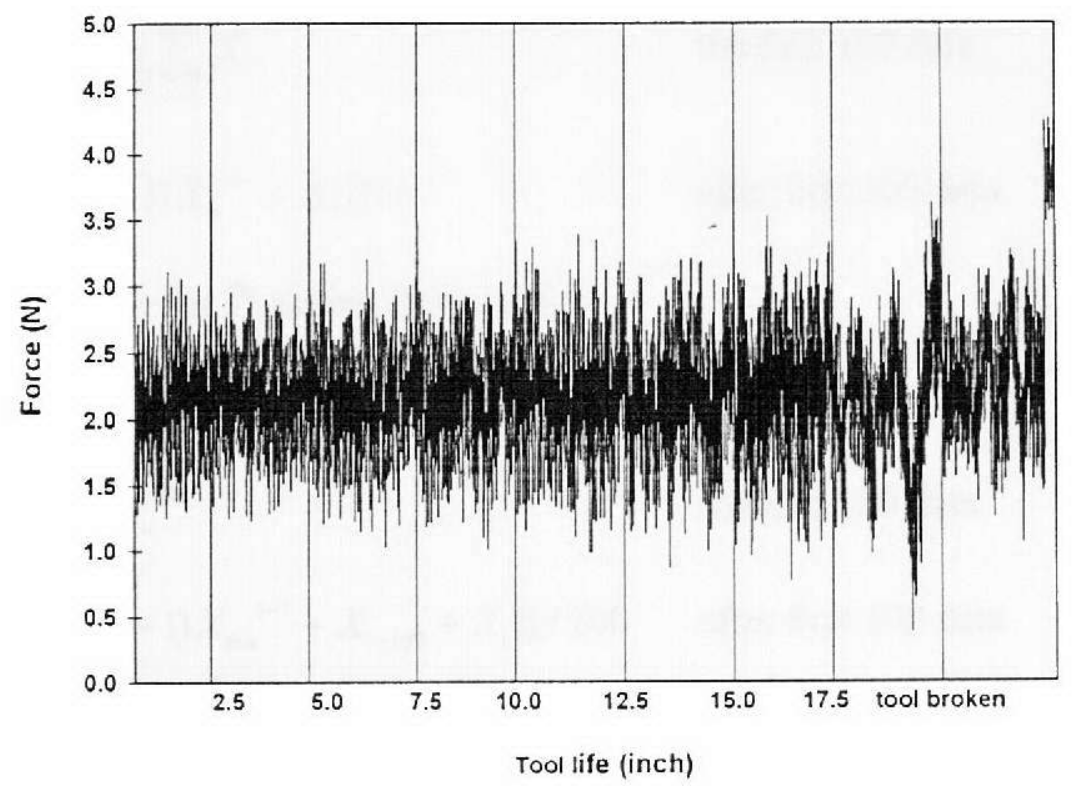

Figure 5.6 Tool cutting force of micro-end-milling experiment II 
speed, 0.004 " depth of cut, $1 \mathrm{ipm}$ feed rate for the mild steel work-piece and $2 \mathrm{ipm}$ feed rate for the aluminum work-piece. The results are presented in Figure 5.5 and 5.6, in which the tool life is segmented in 5 and 9 different segments.

A high frequency cutting force signal with a lower frequency vibration can be observed in the figures. The high frequency signal represents the tool cutting force, whose frequency depended on the machining spindle speed. The lower frequency variation is created by the tool vibration. The vibrations are created due to the inconsistency in the feed rate and the non-homogeneous characteristics of the work-piece.

To detect the tool breakage and estimate its reason, the following five statistical methods were used to process the cutting force data.

1. Cutting force average

$$
\begin{array}{ll}
X_{a}^{100}=\frac{1}{100} \sum_{i=1}^{100} X_{i} & \text { the first } 100 \text { data } \\
\left.X_{a}^{i}=\left[(i-1) X_{a}^{i-1}+X_{i}\right)\right] / i & \text { after first } 100 \text { data }
\end{array}
$$

where: $X_{i}$ is the $i^{\text {th }}$ cutting force data.

2. Cutting force moving average

$$
\begin{array}{ll}
X_{m a}^{100}=X_{a}^{100} & \text { the first } 100 \text { data } \\
\left.X_{m a}{ }^{i}=\left[(i-1) X_{m a}^{i-1}-X_{i-100}+X_{i}\right)\right] / 100 & \text { after first } 100 \text { data }
\end{array}
$$

3. Cutting force variation

$$
\begin{array}{ll}
X_{v}^{100}=\frac{1}{100} \sum_{i=1}^{100}\left(X_{i}-X_{a}^{100}\right)^{2} & \text { the first } 100 \text { data } \\
X_{v}^{i}=\left[(i-1) X_{v}^{i-1}+\left(X_{i}-X_{a}^{i}\right)^{2}\right] / i & \text { after first } 100 \text { data }
\end{array}
$$


4. Cutting force moving variation

$$
\begin{array}{ll}
X_{m v}{ }^{100}=X_{v}{ }^{100} & \text { the first } 100 \text { data } \\
X_{v}{ }^{i}=\left[(i-1) X_{m v}{ }^{i-1}+\left(X_{i}-X_{m a}{ }^{i}\right)^{2}\right] / i & \text { after first } 100 \text { data }
\end{array}
$$

5. Variation of cutting force moving averages

$$
\begin{array}{ll}
X_{\text {mav }}^{100}=0 & \text { the first } 100 \text { data } \\
X_{\text {mav }}{ }^{i}=\left[(i-100) X_{a n v}{ }^{i-1}+\left(X_{m a}{ }^{i}-X_{a}^{i}\right)^{2}\right] /(i-100+1) & \text { after first } 100 \text { data }
\end{array}
$$

The cutting force variation is the main indictor of the tool breakage. When it becomes larger than a tool breakage critical value, the tool would create a poor machined surface and break very soon. The cutting force moving variation is related to the tool wear level. If it is larger than a tool wear critical value, the tool is really worn and shouldn't be used any more. The cutting force moving average and its variation provides the tool vibration information. The vibrations could cause rough surface, short tool life or tool breakage. If the variation of the cutting force moving average is larger than a tool vibration critical value and the cutting force moving variation is still less than the tool wear critical value, the tool life could be extended by reducing the feed rate or cleaning it immediately.

The test results of two experimental cases are presented in Figure 5.7 to 5.14. The 0.015 " diameter tool used with the mild steel work-piece had almost 3.5 " tool life. The 0.015 " diameter tool cutting the aluminum work-piece had almost 20 " tool life.

During the machining of mild steel work-piece, the cutting force average was remained around $0.2 \mathrm{~N}$ (see Figure 5.7 and 5.8) and moving average was remained around $\pm 0.2 \mathrm{~N}$. The tool worked in a good condition with a very little vibration. The cutting force 
was gradually increased while it was worn. The tool cutting force variation was gradually increased from 0.2 to 1.0 (see Figure 5.10) until reached the tool breakage critical value. The reason of the tool breakage was the tool wear.

Compared to the mild steel work-piece, the experiment data of the aluminum work-pork-piece had very different characteristics. The cutting force average was remained around $2.2 \mathrm{~N}$ (see Figure 5.11 and 5.12). The cutting force variation remained in the same level $0.15 \mathrm{~N}$ (see Figure 5.13 and 5.14). The tool cutting force changed very little, but its vibration continuously increased. The moving average changed from a small vibration $\pm 0.1 \mathrm{~N}$ for the new tool (see Figure 5.11) to a large vibration $\pm 1.0 \mathrm{~N}$ for the worn tool just before its breakage (see Figure 5.14). The significant change was observed around $2300^{\text {th }}$ data point (see Figure 5.14). The variation of the cutting force moving average rapidly increased. This increase caused the cutting force variation to increase over the tool breakage critical value.

The main reason of the tool breakage during the machining the aluminum workpiece is the vibration, not the tool wear. Since aluminum has a lower milting point, some melted chips might have stuck on the tool. The cutting edges lost their sharpness and vibrations were created. Finally tool life could be extended if the cutting edges were allowed to be cleaned down by reducing the feed rate.

Tool breakage and vibration critical values of the proposed algorithms should be determined from the experimental study. 
New Micro-tool Cutting Force Average

(mild steel work-piece)

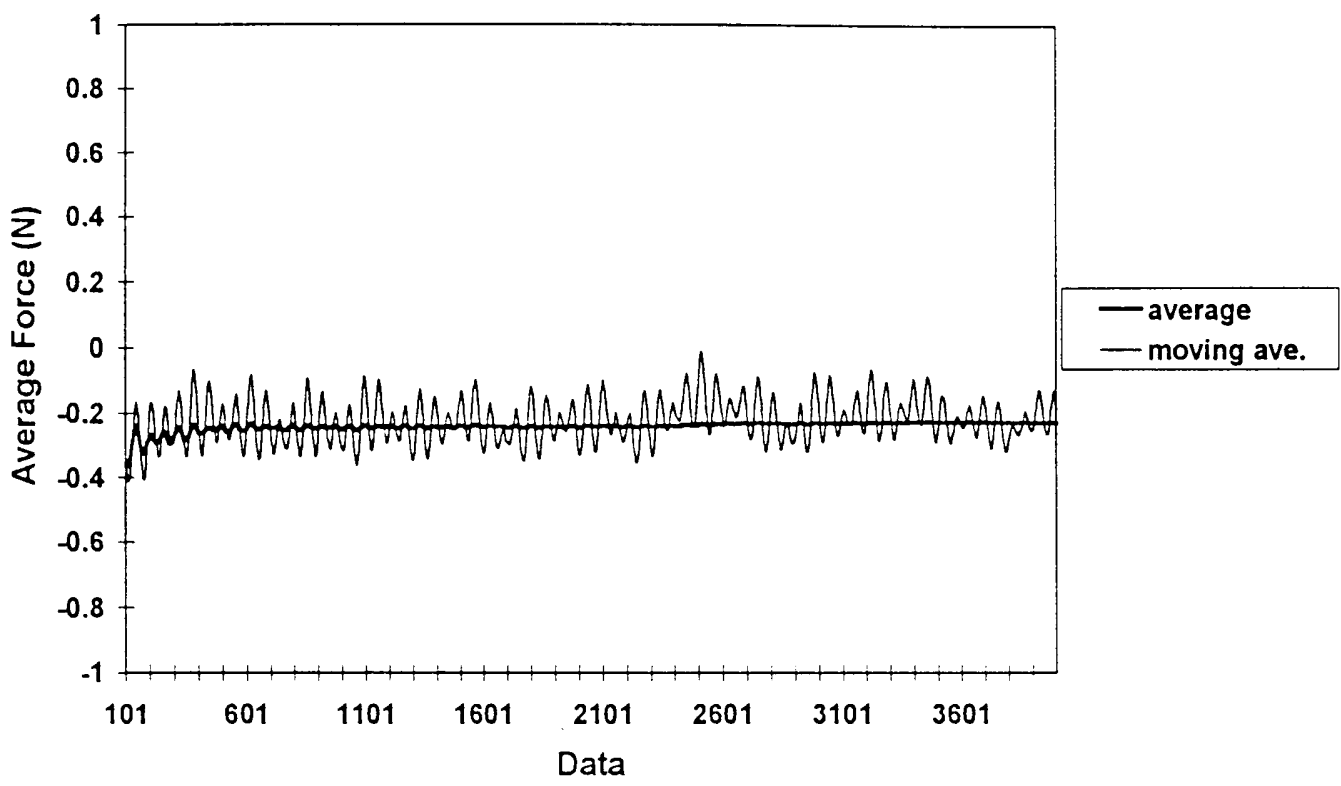

Figure 5.7 New tool cutting force average of experiment I

Micro-tool Cutting Force Average Before Breakage (mild steel work-piece)

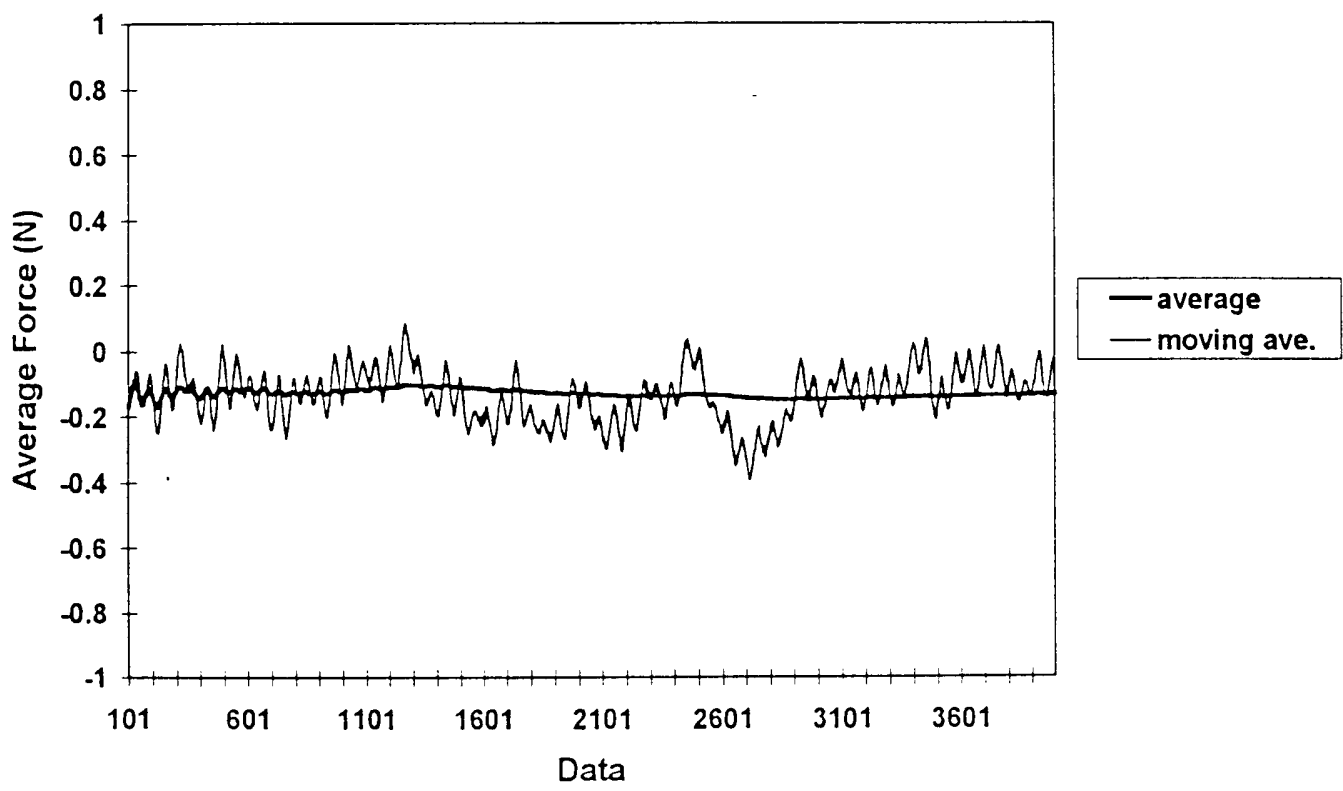

Figure 5.8 Tool cutting force average before breakage of experiment I 
New Micro-tool Cutting Force Variation

(mild steel work-piece)

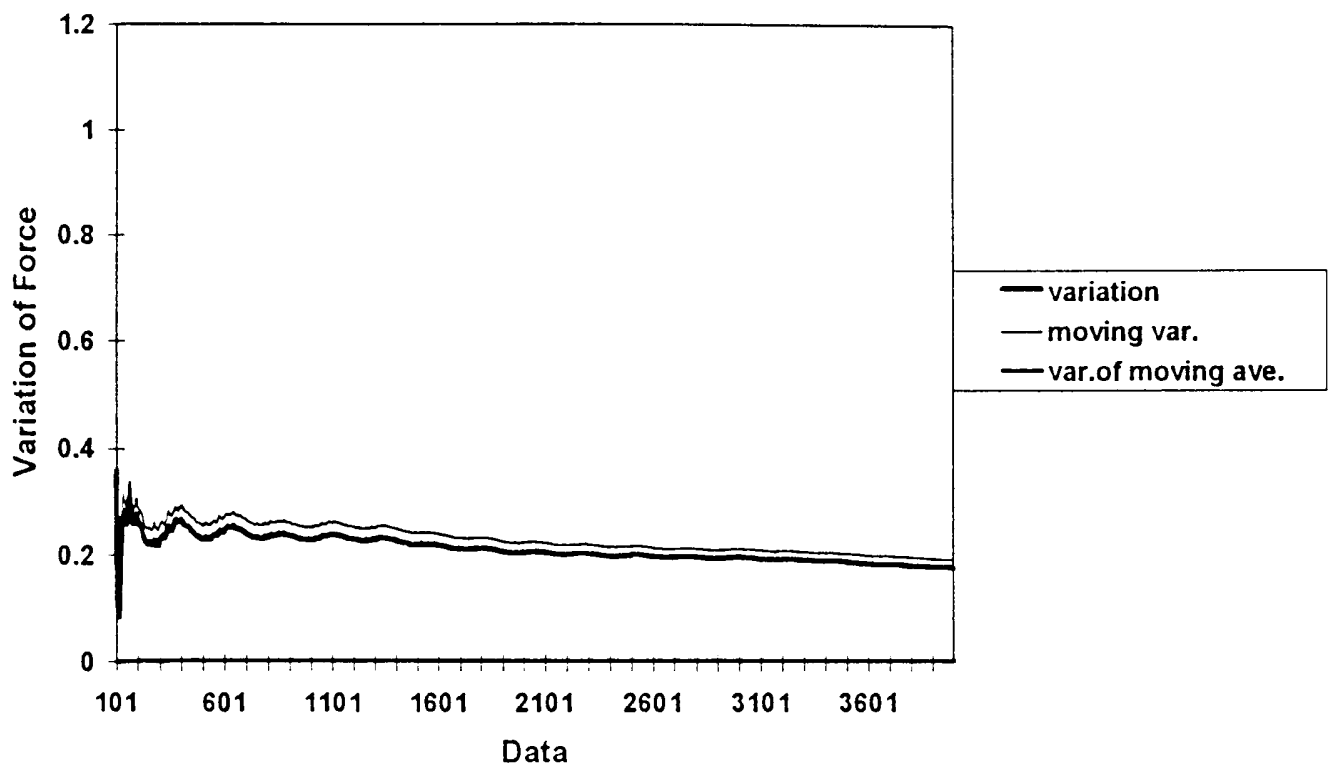

Figure 5.9 New tool cutting force variation of experiment I

Micro-tool Cutting Force Variation Before Breakage

(mild steel work-piece)

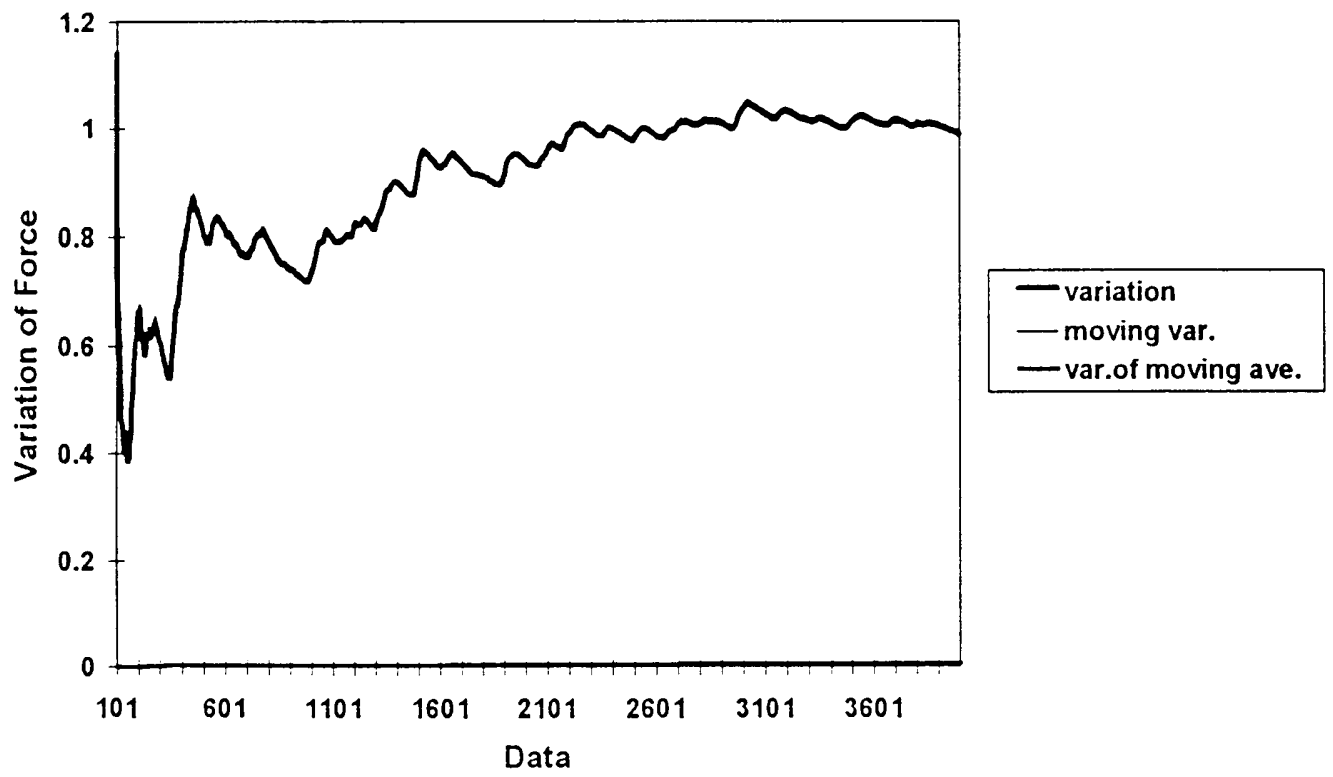

Figure 5.10 Tool cutting force variation before breakage of experiment I 
New Micro-tool Cutting Force Average

(aluminum work-piece)

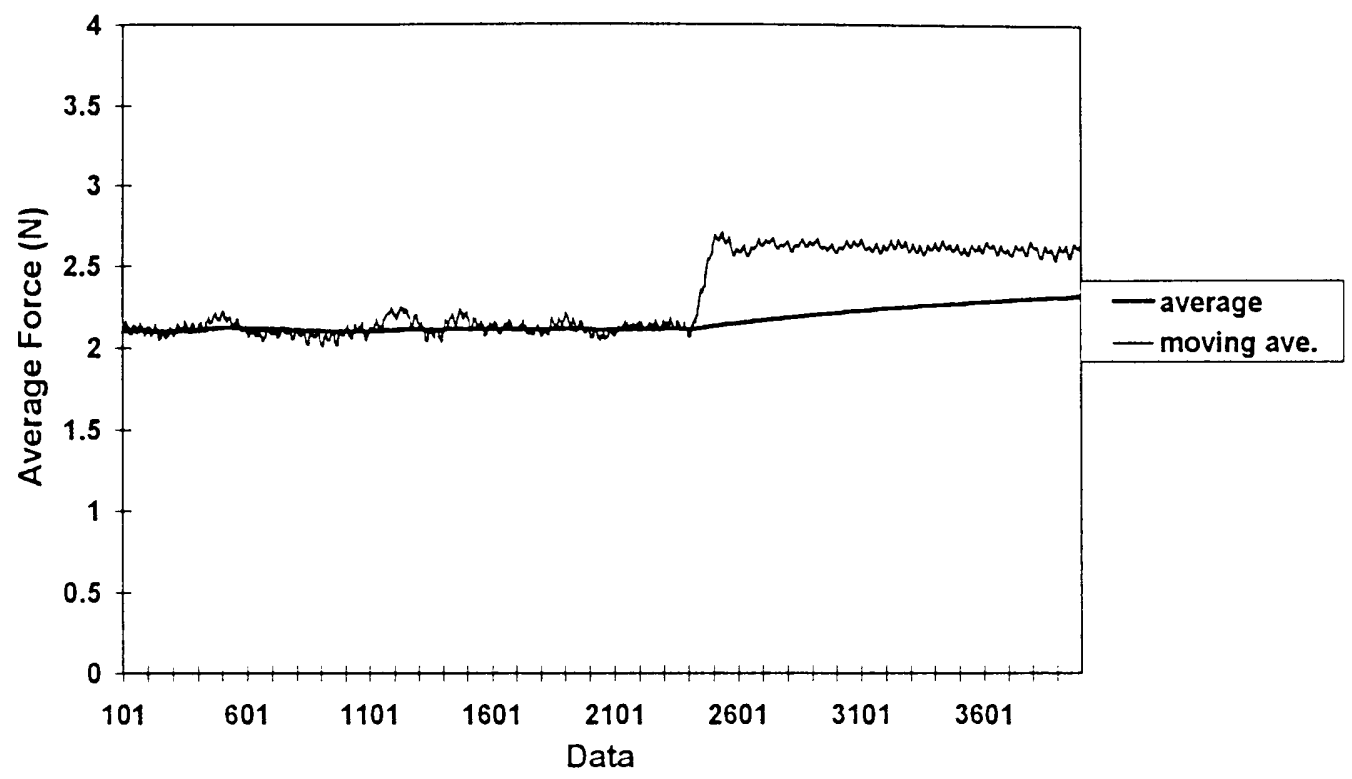

Figure 5.11 New tool cutting force average of experiment II

Micro-tool Cutting Force Average Before Breakage (aluminum work-piece)

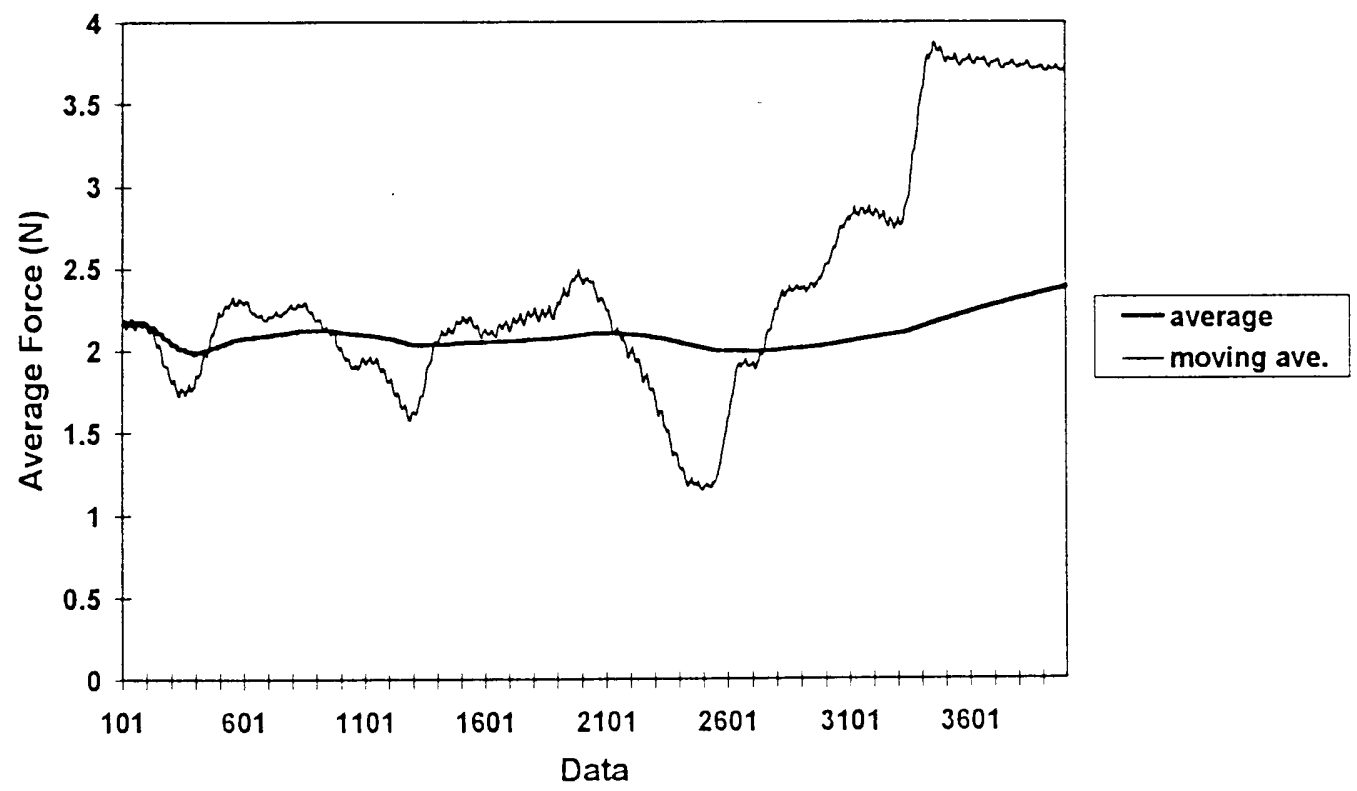

Figure 5.12 Tool cutting force average before breakage of experiment II 


\section{New Micro-tool Cutting Force Variation}

(aluminum work-piece)

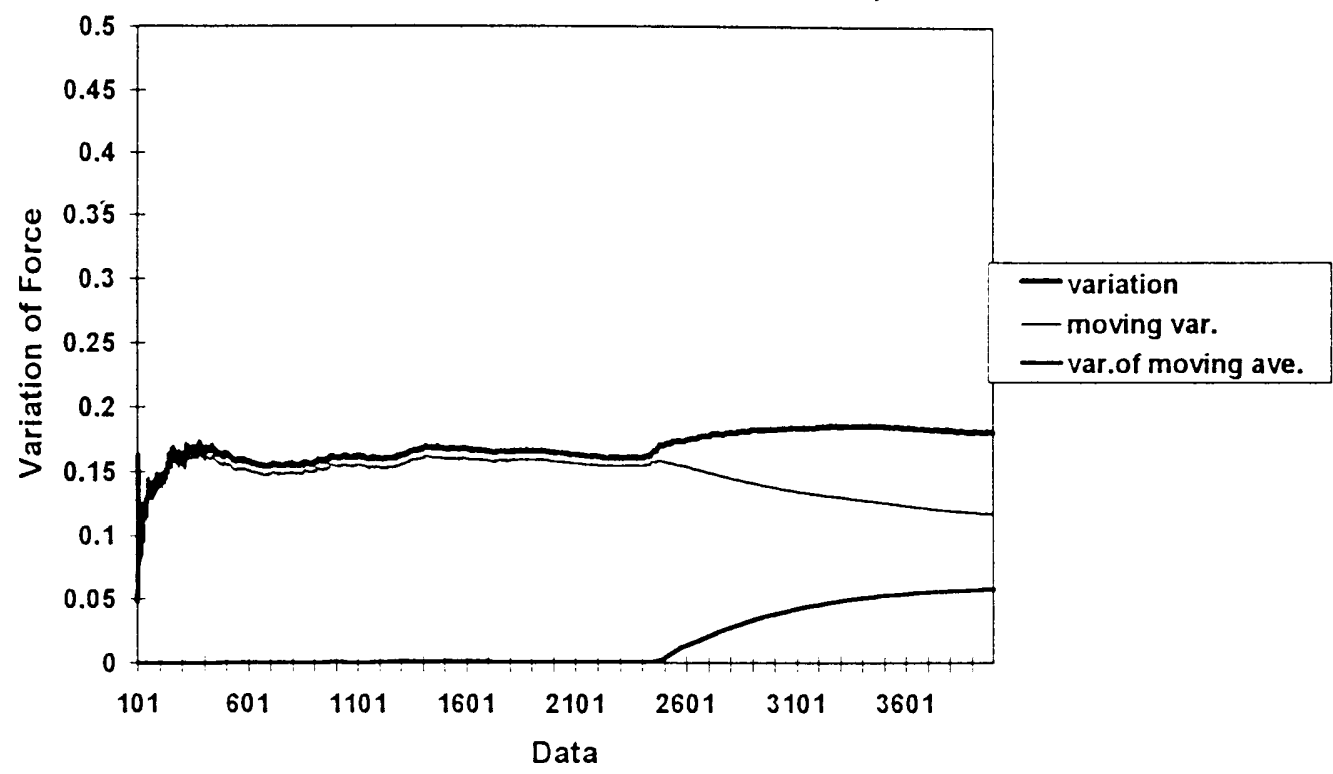

Figure 5.13 New tool cutting force variation of experiment II

\section{Micro-tool Cutting Force Variation Before Breakage (aluminum work-piece)}

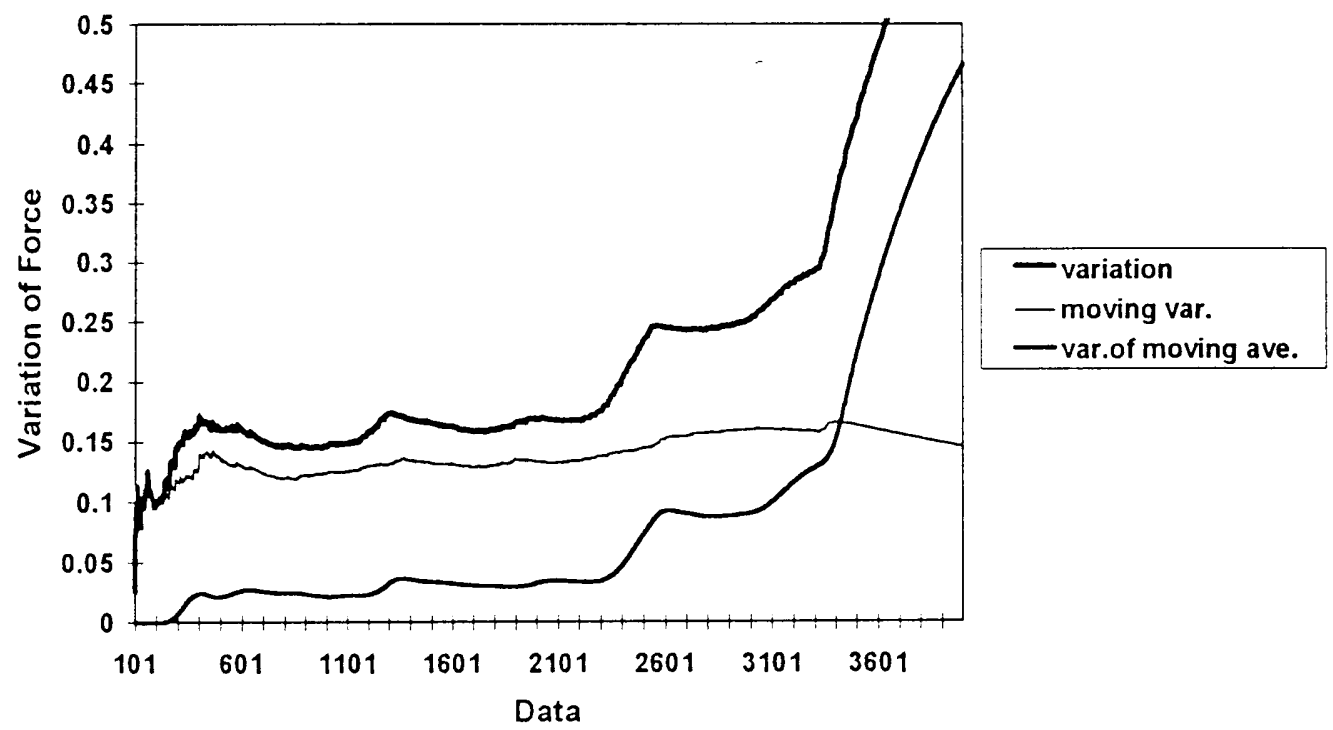

Figure 5.14 Tool cutting force variation before breakage of experiment II 


\subsection{Tool Wear Estimation}

Many researchers have studied the characteristics of cutting force to relate them to tool wear. To simplify the estimation algorithm, to reduce the cost of the instrumentation, and to increase the reliability of the system, the following assumptions can be made:

Assumption 1: The tool works in an identical cutting condition.

Assumption 2: The tool cutting force depends on the tool wear only.

In this section, the relationship of cutting force and tool wear will be studied and a tool wear estimation method will be proposed.

\section{Relationship of tool wear and cutting force ${ }^{[42]}$}

It has been known that cutting forces of micro-end-milling operations increase with tool wear. For most of metals, the cutting forces can be measured with conventional dynamometers. For some non-metal material work-pieces, like POCO-3 and POCO-C3, the small cutting force is almost at the same level with the noises created by the inertia force of the dynamometer. To evaluate tool condition, the cutting forces were collected while an aluminum work-piece was cut periodically.

The experiments were performed on a 3-axis 15,000 rpm Fadal CNC Machine in Engineering Prototype Center of Radio Technology Division of Motorola Inc. A POCOC3 work-piece was attached onto a Kistler 9257B dynamometer that was installed on the table of the machine tool. On the top of the work-piece, a small aluminum test work-piece was attached. The tool cutting force signals were monitored and collected by a Nicolet 310 two-channel digital oscilloscope through a Kistler three channel charge amplifier. To 
control the experimental machining operation, the $\mathrm{CNC}$ machine was programmed by using Smart CAM Version 10 Production Milling software. The experimental setup will be presented in Chapter VI.

Two different work-pieces, steel and POCO-C3, were tested. In the machining of steel work-pieces, cutting force was collected during the machining operations. Two-flute carbide end mills with 0.030 " diameter were used in the experiments. The working conditions were $30,000 \mathrm{rpm}$ spindle speed, $2.5 \mathrm{ipm}$ feed rate and 0.015 " depth of cut for the experiment I; and 20,000 rpm spindle speed, $1.25 \mathrm{ipm}$ feed rate and 0.0225 " depth of cut for the experiment II. The experimental results are presented in Figure 5.15 and 5.16, which includes 14 and 10 data points starting from new tools and ending until they were broken. The tools had around 42" and 33" tool life in the experiment I and II respectively.

To evaluate the cutting force characteristics and tool wear relationship of nonmetal POCO-C3 work-piece, periodic test cuttings were done on the aluminum test workpiece during the machining of POCO-C3. Two-flute carbide end mills with 0.030" diameter were used. The working conditions of the POCO-C3 machining operations were $15,000 \mathrm{rpm}$ spindle speed, $20 \mathrm{ipm}$ feed rate and 0.030 " depth of cut. The working conditions of the machining of the aluminum test work-piece were 5,000 rpm spindle speed, $5 \mathrm{ipm}$ feed rate and 0.015 " depth of cut. The experimental results are presented in Figure 5.17, which includes 9 data points starting from a new tool and ending until it was broken. The tool had around 1350" tool life. That non-metal material work-piece had an excellent machinability. 
Monitoring Tool Wear by Cutting Force(thrust direction)

two flute $0.030^{\prime \prime}$ diameter carbide end mill, steel work-piece

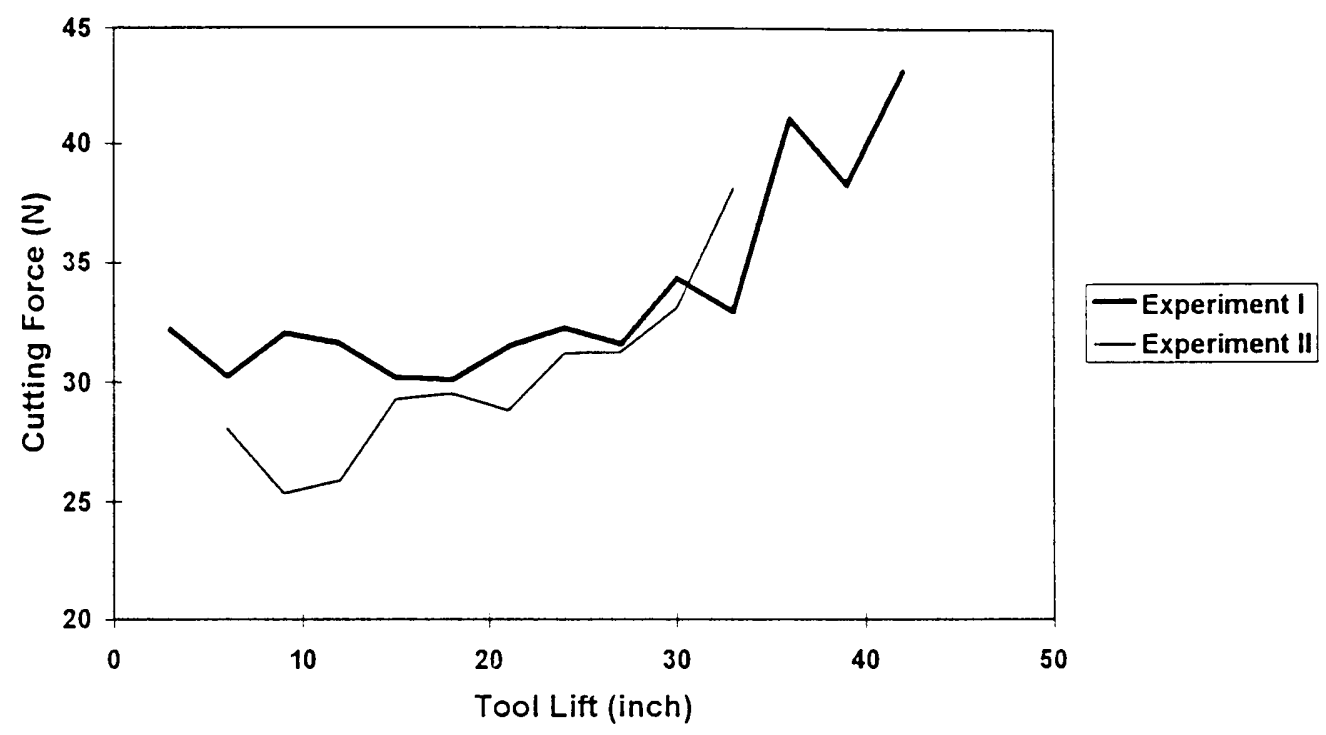

Figure 5.15 Monitoring of tool wear by the thrust direction cutting force

Monitoring Tool Wear by Cutting Force (feed direction)

two flute 0.030 " diameter carbide end mill, steel work-piece

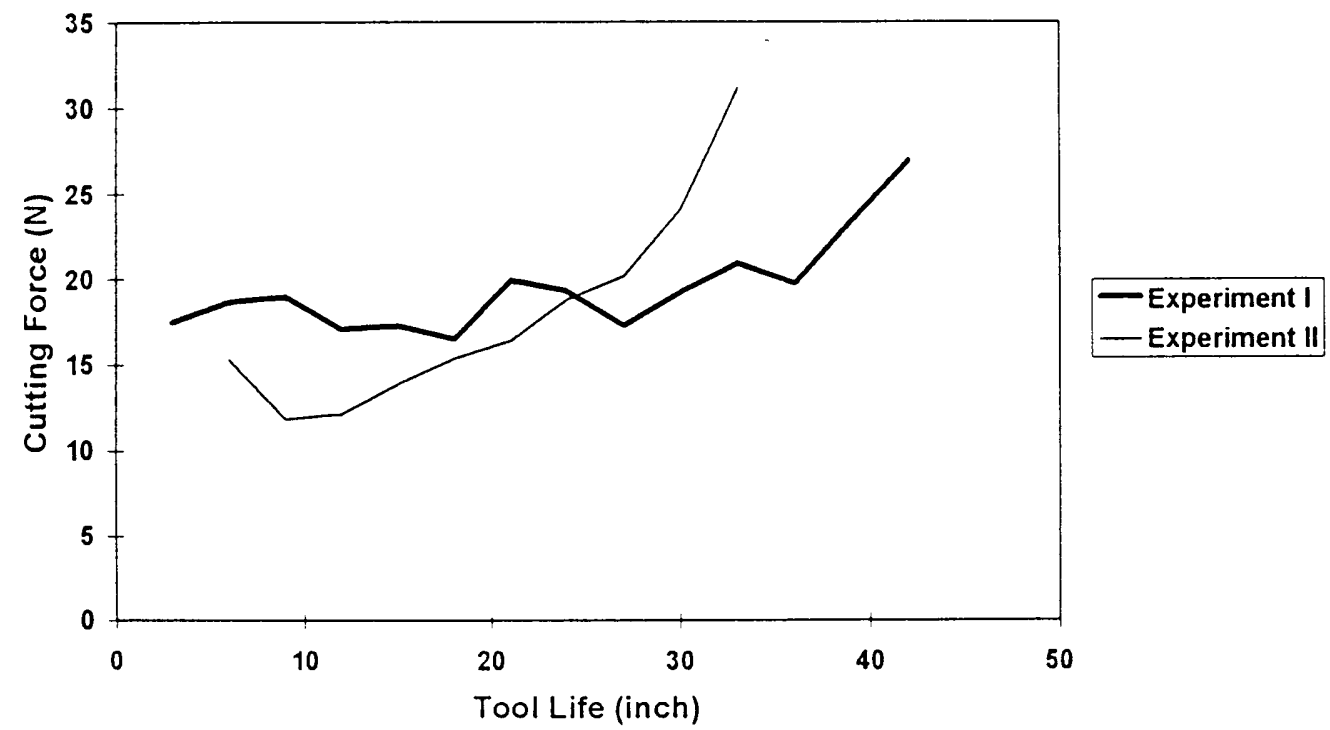

Figure 5.16 Monitoring of tool wear by the feed direction cutting force 


\section{Monitoring Tool Wear by Cutting Force}

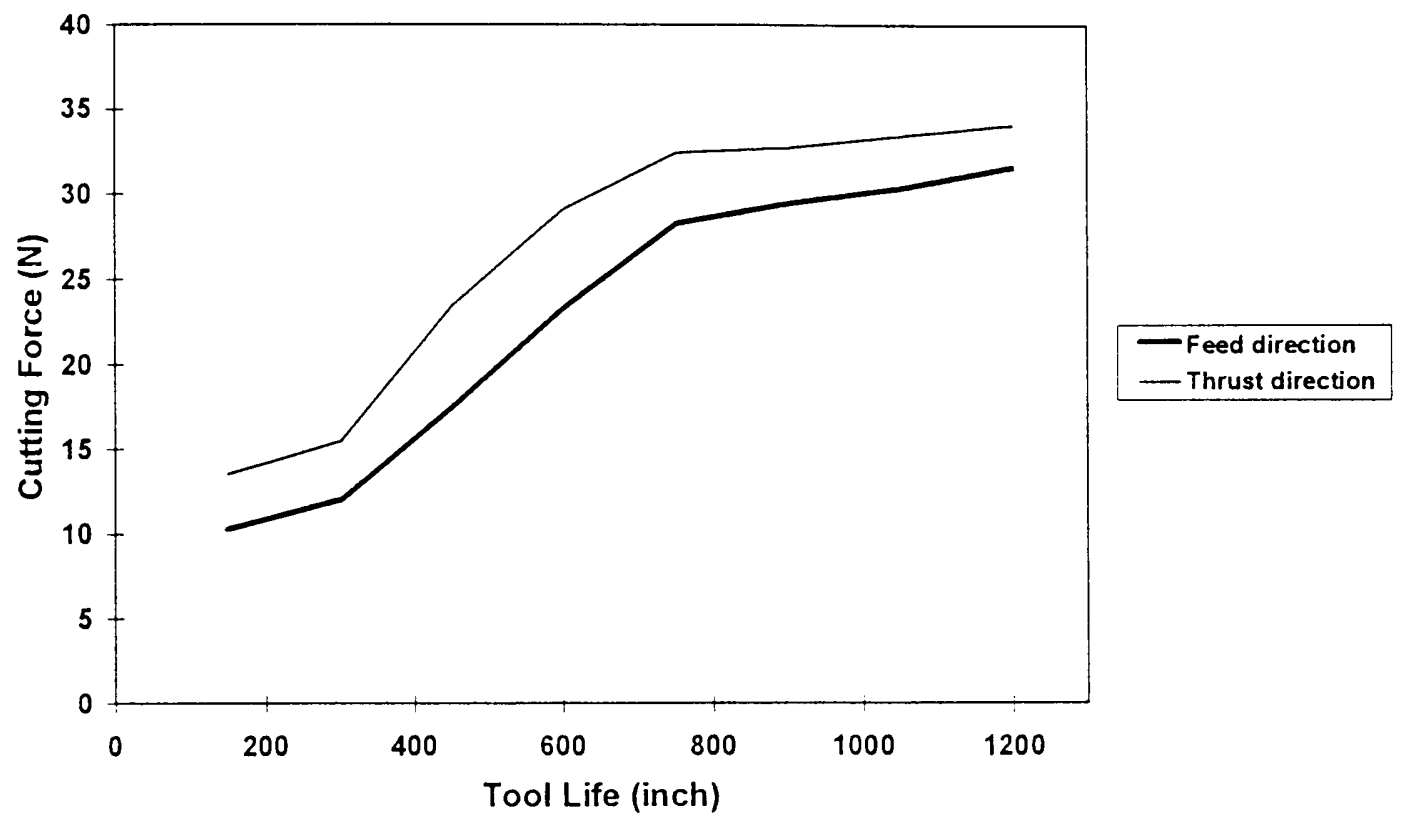

Figure 5.17 Indirect monitoring of tool wear by the cutting forces 


\section{Estimation of tool wear by using genetic algorithms}

Based on the tool wear and cutting force relationship study, the empirical tool wear model is proposed in the following format.

$$
\mathrm{F}_{\max }=\mathrm{C}_{1}+\left(\mathrm{C}_{2} * \mathrm{~L}\right)^{\mathrm{C} 3}
$$

where: $F_{\max }$ is tool maximum cutting force $(N)$

$$
\begin{aligned}
& \mathrm{L} \text { is tool life (inch) } \\
& \mathrm{C}_{1}, \mathrm{C}_{2} \text { and } \mathrm{C}_{3} \text { are coefficients. }
\end{aligned}
$$

In the empirical tool wear model, three coefficients have their physical meaning. $C_{1}$ is the basic cutting force level, which depends on the working conditions of micro-endmilling operation. $\mathrm{C}_{2}$ is the tool wear gradient, which depends on the cutting conditions, the tool and work-piece materials. A small $\mathrm{C}_{2}$ indicates slow progress of the wear. $\mathrm{C}_{3}$ presents how fast the tool is broken when the cutting force is above a critical level. The harder the work-piece material is or the more uncomfortable cutting conditions the tool has, the larger $C_{3}$ is.

From the experimental data of the tool wear, the coefficients of the proposed tool wear model can be found by using the genetic algorithms (see Chapter II). The program GATool (Genetic Algorithm Research Tool) used in the research was developed in 1998 and successfully applied to the tool cutting condition monitoring. ${ }^{[19]}$ GATool is used to search the optimal fitting coefficients of the tool wear model. The sum of the difference between the experimental cutting force data and the cutting force obtained from the developed analytical model is used as the optimal objective function. 
Objective function:

$\operatorname{Min}(E)=\frac{1}{N} \sum_{i=1}^{N}\left|F_{\max }^{\text {estimated }}(i)-F_{\max }^{\text {actual }}(i)\right|$

where: $E$ is the average absolute error.

$\mathrm{N}$ is the number of the experimental data.

$F_{\max }^{\text {actual }}(i)$ and $F_{\max }^{\text {estmated }}(i)$ are the experimental and estimated maximum cutting forces in the i" tool life.

In the genetic evolution procedures, the 30-bit binary coding is used for three coefficients by assigning 10 bits for each one. The population size was selected as five. Mating pool size was two versus two and one child from each couple. The uniform crossover (with 0.5 probability), jumping mutation (with 0.1 probability), creeping mutation (with 0.05 probability) and elitism are chosen. The 14 data sets of the tool wear experiment I (see Figure 5.15, experiment I) were used in the study.

The optimal coefficients of the tool wear model were found in the $500^{\text {th }}$ generation with $\pm 1.1 \mathrm{~N}$ or $3.3 \%$ error.

$$
\begin{aligned}
& C_{1}=30.968 \\
& C_{2}=0.0423 \\
& C_{3}=4.352
\end{aligned}
$$

The genetic evolution procedure of the tool wear model is presented in Figure 5.18. The empirical tool wear model is presented in Figure 5.19. 


\section{Genetic Evolution Procedure}

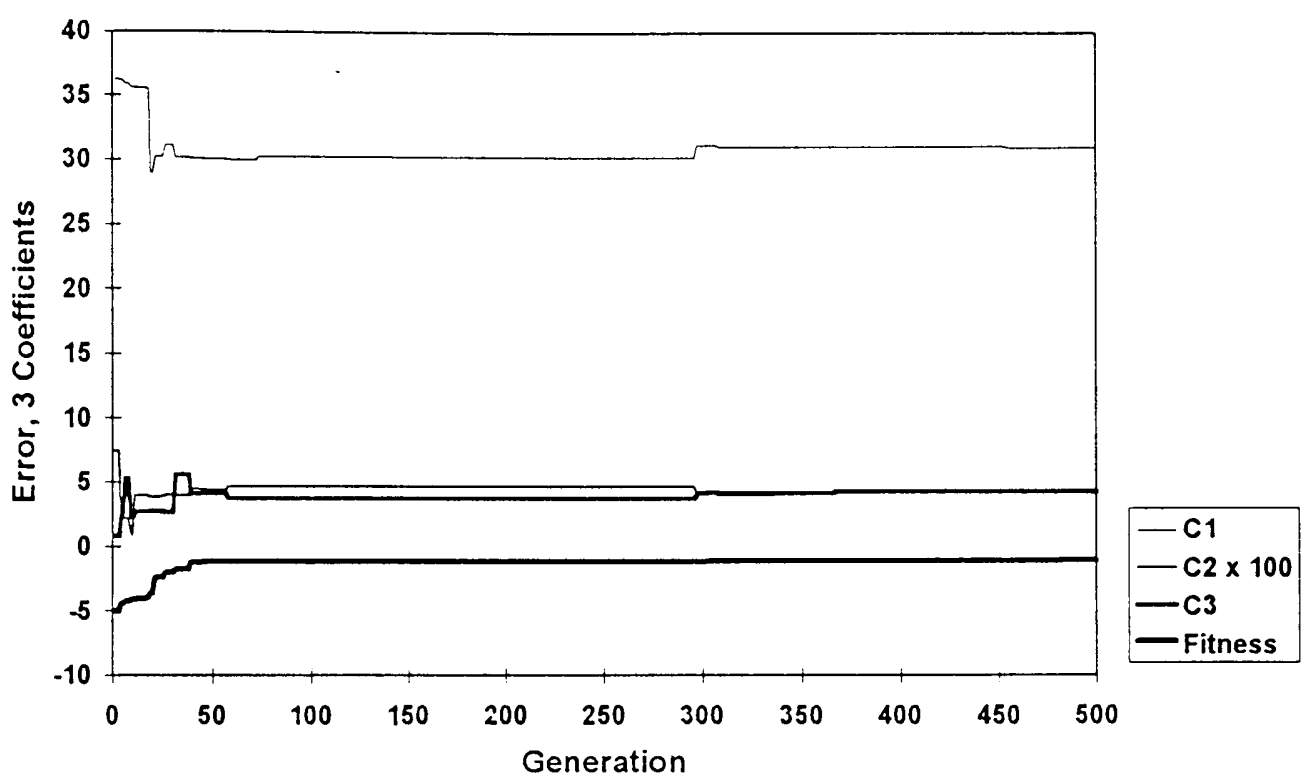

Figure 5.18 Genetic evolution procedure of the tool wear model

Tool Wear Empirical Model

2 flute $0.030^{\prime \prime}$ diameter carbide end mill, steel work-piece

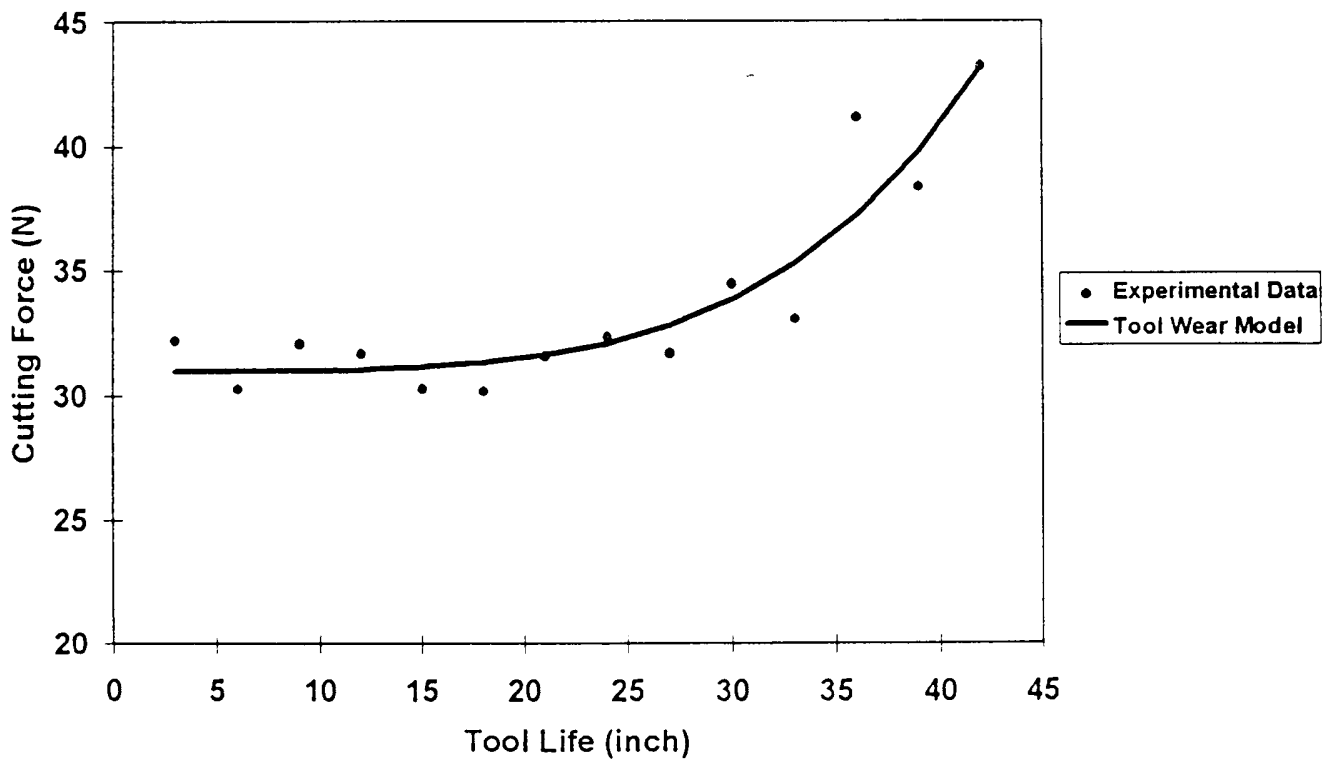

Figure 5.19 An empirical tool wear model 
3. Tool wear estimation by using neural network forecasting model ${ }^{[39[[44][45]}$

Back-propagation neural network with time series type input (see Chapter II) is proposed to estimate the tool wear. The program NNTool (Neural Network Research Tool) used in the studies was developed in 1995 and modified in 1996. Its forecasting capability has been proved in many cases. ${ }^{[15]}$

In this study, the two input and one output back-propagation neural network was used. The inputs were the present and one-step prior cutting force data and output was the one-step ahead cutting force data. A three-layer neural network with seven hidden nodes was designed. The learning rate and momentum factor were selected as 0.15 and 0.9 respectively. Out of the 14 data sets of the tool wear experiment I (see Figure 5.15, experiment I), The 12 data sets were used for the training of the neural network.

The tool wear estimation neural network model had been generated in 10,000 iterations. Compared to the empirical tool wear model and the experimental data, the tool wear estimation model had $0.4 \%$ and $3.3 \%$ error respectively. The results are presented in Table 5.1 and Figure 5.20. The future cutting force could be forecast by the tool wear estimation neural network model. By comparing the estimated cutting force to the maximum allowable cutting force, the tool life could be estimated.

The tool wear coefficient $K_{w}$ of the analytical cutting force model can be obtained by using the following equation:

$$
\mathrm{K}_{\mathrm{w}}=\mathrm{F}_{\max } / \mathrm{C}_{1}
$$




\begin{tabular}{|c|c|c|c|c|c|c|}
\hline & \multicolumn{6}{|c|}{ Cutting Force (N) } \\
\hline $\begin{array}{c}\text { Tool Life } \\
\text { inch) }\end{array}$ & $\begin{array}{c}\text { Experimental } \\
\text { Data }\end{array}$ & $\begin{array}{c}\text { Empirical } \\
\text { Model }\end{array}$ & $\begin{array}{c}\text { Estimation } \\
\text { Model }\end{array}$ & $\begin{array}{c}\text { Empirical } \\
\text { Model Error }\end{array}$ & $\begin{array}{c}\text { Estimation } \\
\text { Model Error }\end{array}$ & $\begin{array}{c}\text { Error between } \\
\text { Both Models }\end{array}$ \\
\hline 3 & 32.200 & 30.968 & & 1.232 & & \\
\hline 6 & 30.240 & 30.971 & & -0.731 & & \\
\hline 9 & 32.040 & 30.983 & 31.204 & 1.057 & 0.836 & -0.221 \\
\hline 12 & 31.640 & 31.020 & 31.215 & 0.620 & 0.425 & -0.195 \\
\hline 15 & 30.220 & 31.106 & 31.252 & -0.886 & -1.032 & -0.146 \\
\hline 18 & 30.120 & 31.273 & 31.346 & -1.153 & -1.226 & -0.072 \\
\hline 21 & 31.520 & 31.565 & 31.542 & -0.045 & -0.022 & 0.023 \\
\hline 24 & 32.280 & 32.036 & 31.907 & 0.244 & 0.373 & 0.129 \\
\hline 27 & 31.640 & 32.751 & 32.530 & -1.111 & -0.890 & 0.221 \\
\hline 30 & 34.400 & 33.788 & 33.534 & 0.612 & 0.867 & 0.255 \\
\hline 33 & 33.000 & 35.238 & 35.061 & -2.238 & -2.061 & 0.176 \\
\hline 36 & 41.120 & 37.203 & 37.238 & 3.917 & 3.882 & -0.034 \\
\hline 39 & 38.320 & 39.802 & 40.036 & -1.482 & -1.716 & -0.234 \\
\hline 42 & 43.200 & 43.164 & 43.107 & 0.036 & 0.093 & 0.057 \\
\hline Average & 33.710 & 33.705 & 29.284 & 1.097 & 1.119 & 0.147 \\
\hline Percentage & & & & $3.26 \%$ & $3.32 \%$ & $0.44 \%$ \\
\hline
\end{tabular}

Table 5.1 Results of the tool wear estimation model

Tool Wear Estimation Model

2 flute $0.030^{\prime \prime}$ diameter carbide end mill, steel work-piece

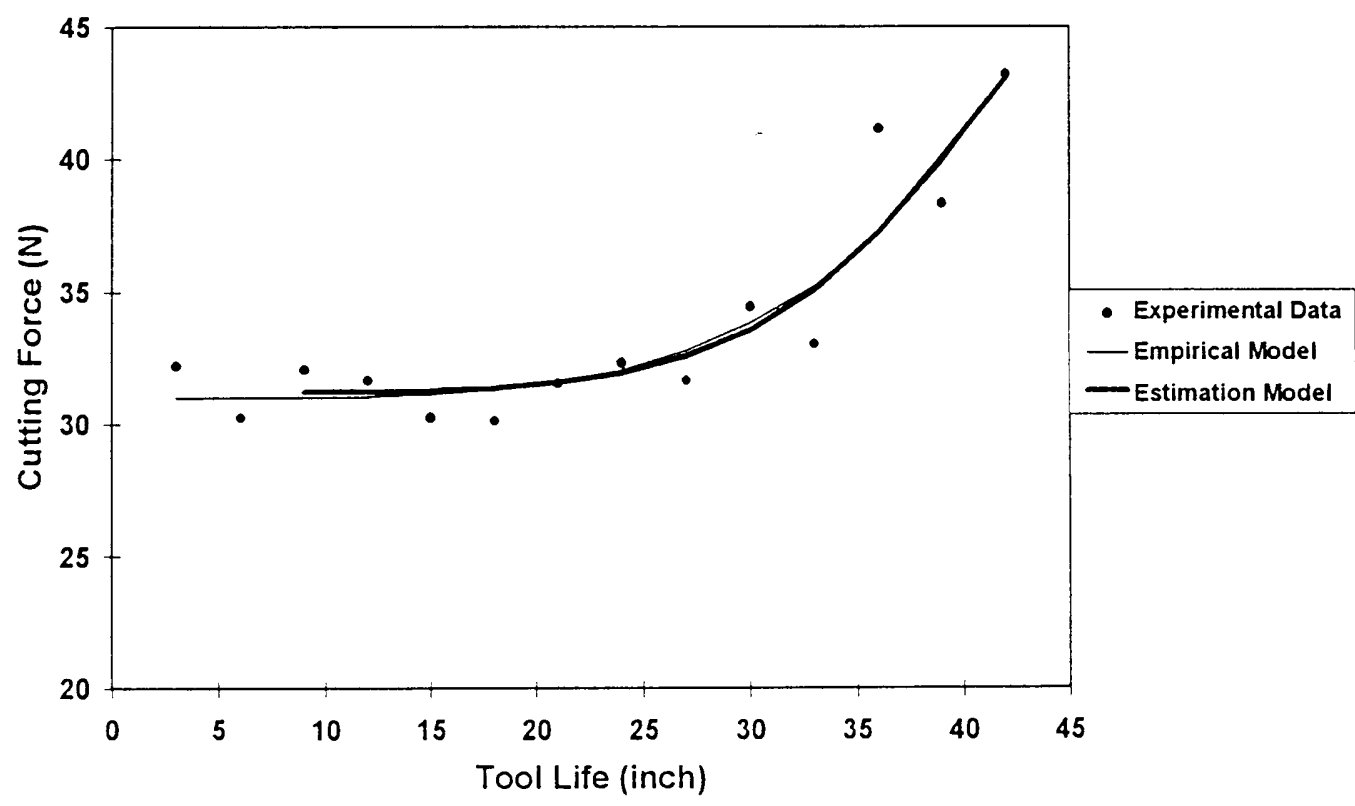

Figure 5.20 Comparison of the tool wear model 


\subsection{Tool Run-out Estimation}

The maximum cutting force of micro-end-milling operations is very different depending on whether the tool has run-out or not. A small tool run-out could significantly increase cutting forces and not favor the surface finish.

From the characteristics of the cutting force, existence of run-out can be easily understood. The maximum cutting forces created by each cutting edge are the main indicators. If they are the same, there is no run-out. If they are different, the tool is working with run-out. The run-out of two-flute end-mill can be easily estimated from the maximum cutting forces of each cutting edge.

Let's consider $\mathrm{x}$-coordinate for the feed direction and $\mathrm{y}$-coordinate for the thrust direction. The tool run-out $r_{o}$ is the distance between the tool center and tool turning center, and tool run-out angle $\gamma$ is the angle between the tool run-out and $y$-axis. In other words, the tool run-out in the $\mathrm{x}$ direction and in the $\mathrm{y}$ direction can be calculated by $\mathrm{r}_{0} \sin \gamma$ and $r_{0} \operatorname{con} \gamma$. The maximum cutting force is a function of tool run-out and its angle if the other machining operation conditions are remained unchanged. It can be expressed as:

$$
F_{\max }=f\left(r_{0}, \gamma\right)
$$

According to the discussion of the cutting force characteristics with tool run-out and angle (see Chapter IV), the tool run-out and angle can not be recognized by only one maximum cutting force value because the maximum cutting force is a multiple value function of the tool run-out and its angle. The maximum cutting forces of both feed and thrust direction are needed for the estimations of tool run-out and its angle by an optimal objective function. 
$\operatorname{Min}(E)=\frac{1}{4}\left(\left|F_{X \max 1}^{\text {actual }}-F_{X \max 1}^{\text {estimated }}\right|+\left|F_{X \max 2}^{\text {actual }}-F_{X \max 2}^{\text {estimated }}\right|+\left|F_{Y \max 1}^{\text {actual }}-F_{Y \max 1}^{\text {estimated }}\right|+\left|F_{Y \max 2}^{\text {actual }}-F_{Y \max 2}^{\text {estimated }}\right|\right)$

where: $\mathrm{E}$ is an absolute error.

$F_{X \max 1}^{a c c u a l}, F_{Y \max 1}^{a c t u a l}, F_{X \max 2}^{a c t u a l}$ and $F_{Y \max 2}^{a c t u a l}$ are the monitoring maximum cutting forces in feed and thrust directions of both cutting edges. It is assumed that cutting force value of the first cutting edge is larger than the value of the second cutting one.

$F_{X \max 1}^{\text {estimated }}, F_{Y \max 1}^{\text {estmated }}, F_{X \max 2}^{\text {estimated }}$ and $F_{Y \max 2}^{\text {estimated }}$ are the estimating maximum cutting forces calculated by the analytic cutting force model in feed and thrust directions of both cutting edges.

The genetic algorithm (see Chapter II) was used to minimize the absolute error of the optimal objective function. 30-bit binary coding was used for individuals that include two factors: tool run-out and its angle ( 15 bits for each ). The population size was selected as five. Mating pool size was two versus two and one child from each couple. The uniform crossover (with 0.5 probability), jumping mutation (with 0.1 probability), creeping mutation (with 0.05 probability) and elitism are chosen.

Tool run-out estimation program is included in the MOGART program (see Chapter VIII). The tool run-out and its angle are estimated according to the given tool geometry, cutting conditions, measured feed and thrust direction maximum cutting forces. The estimated maximum cutting forces can be easily obtained from the analytical cutting force model (see Chapter III). The program estimates the tool run-out and its angle, and 
improves their accuracy generation by generation until they reach the optimal one. The procedure of the tool run-out estimation is presented in Figure 5.21. The program user interface is presented in Figure 8.21 of Chapter VIII.

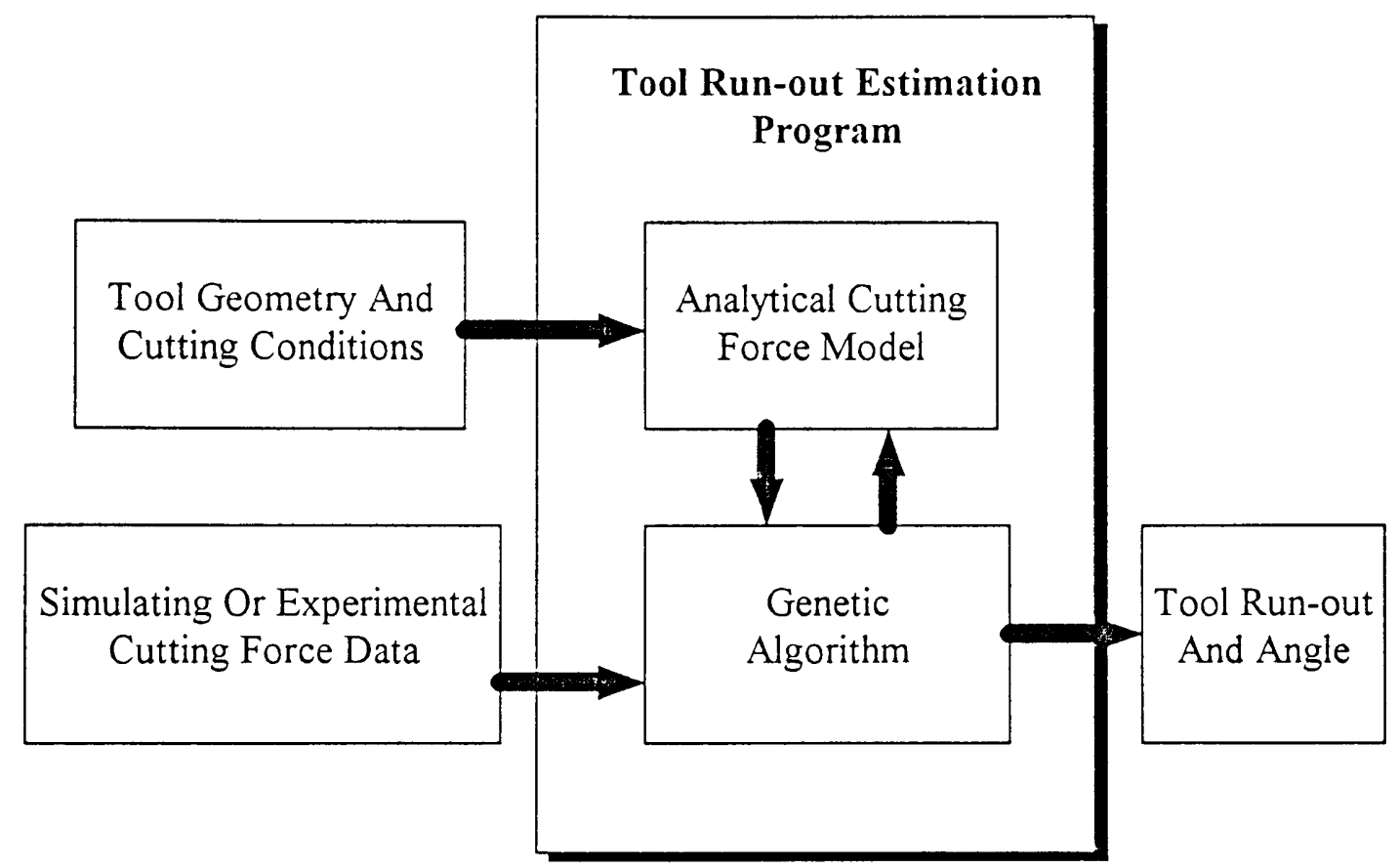

Figure 5.21 Tool run-out estimation procedure

The performance of the tool run-out estimation program was evaluated on a test case. Simulation data was generated by using a two-flute end-mill with 0.020 inch diameter and 45 degree helix angle. The working conditions were $15,000 \mathrm{rpm}$ spindle speed, $120 \mathrm{imp}$ feed rate, 0.010 inch depth of cut, 50\% overlapped climbing milling operations. The material coefficient was considered $80,000 \mathrm{~N} / \mathrm{inch}^{2}$. The tool had a 0.001 inch tool run-out with 30 degree run-out angle. The cutting force variation is presented in Figure 5.22. 
The maximum cutting forces in the feed and thrust directions of both cutting edges are easily obtained from the cutting force profiles. They are:

$$
\begin{array}{ll}
F_{X \max 1}^{\text {actual }}=2.75 \mathrm{~N} & F_{Y \max 1}^{\text {actual }}=4.22 \mathrm{~N} \\
F_{X \max 2}^{\text {actual }}=0.45 \mathrm{~N} & F_{Y \max 2}^{\text {actual }}=1.69 \mathrm{~N}
\end{array}
$$

\section{Cutting Force of Climbing Milling with Tool Run-Out}

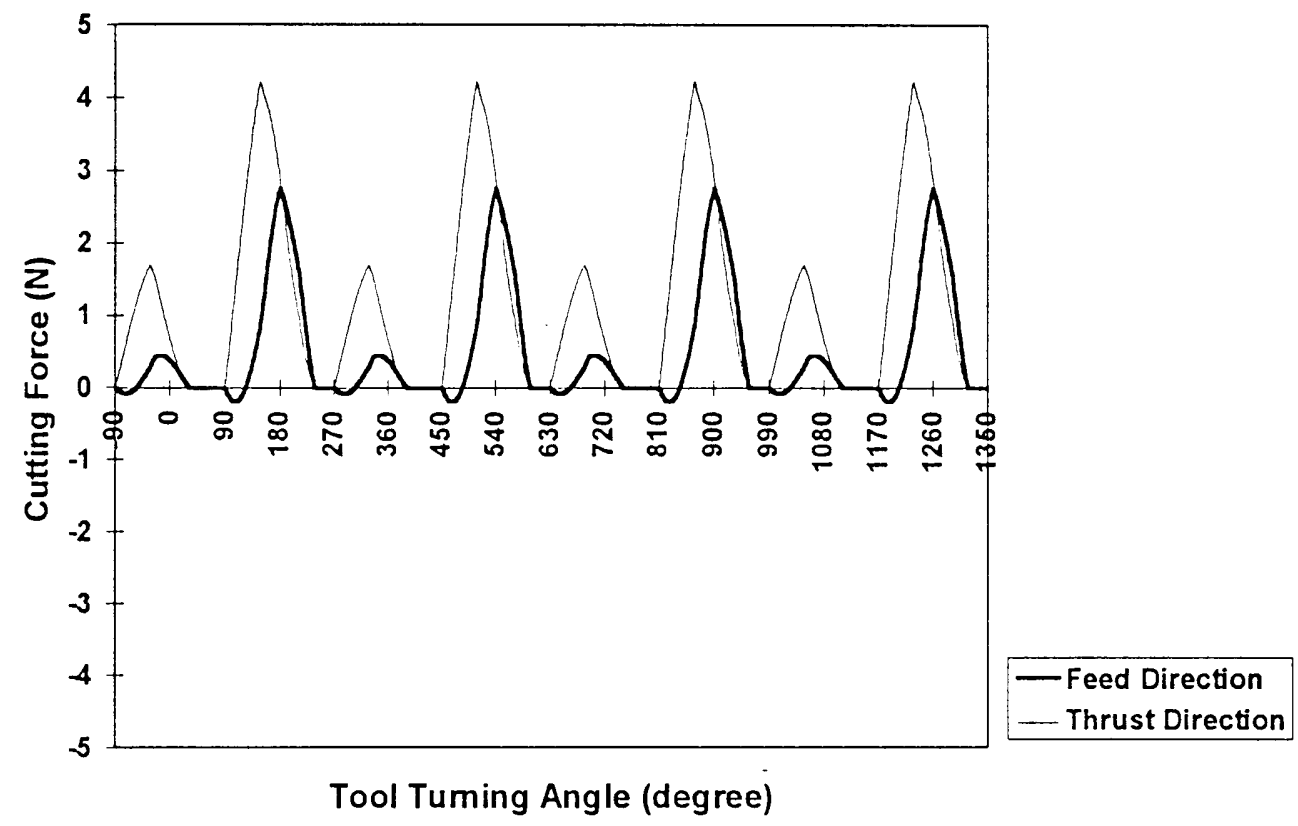

Figure 5.22 Cutting force of climbing milling with tool run-out

In the genetic evolution procedures, the absolute error level of the objective function was selected as 0.005 or $0.5 \%$. The desired error level was reached in the $210^{\text {th }}$ generation with less than $0.32 \%$ error. The estimated tool run-out and its angle were 0.00099 inch and 29.26 degree. Their errors were less than $1 \%$ and $2.5 \%$. The variation of the tool run-out estimation during the optimization process of the genetic algorithm is presented in Figure 5.23. 
Genetic Evolution Procedure

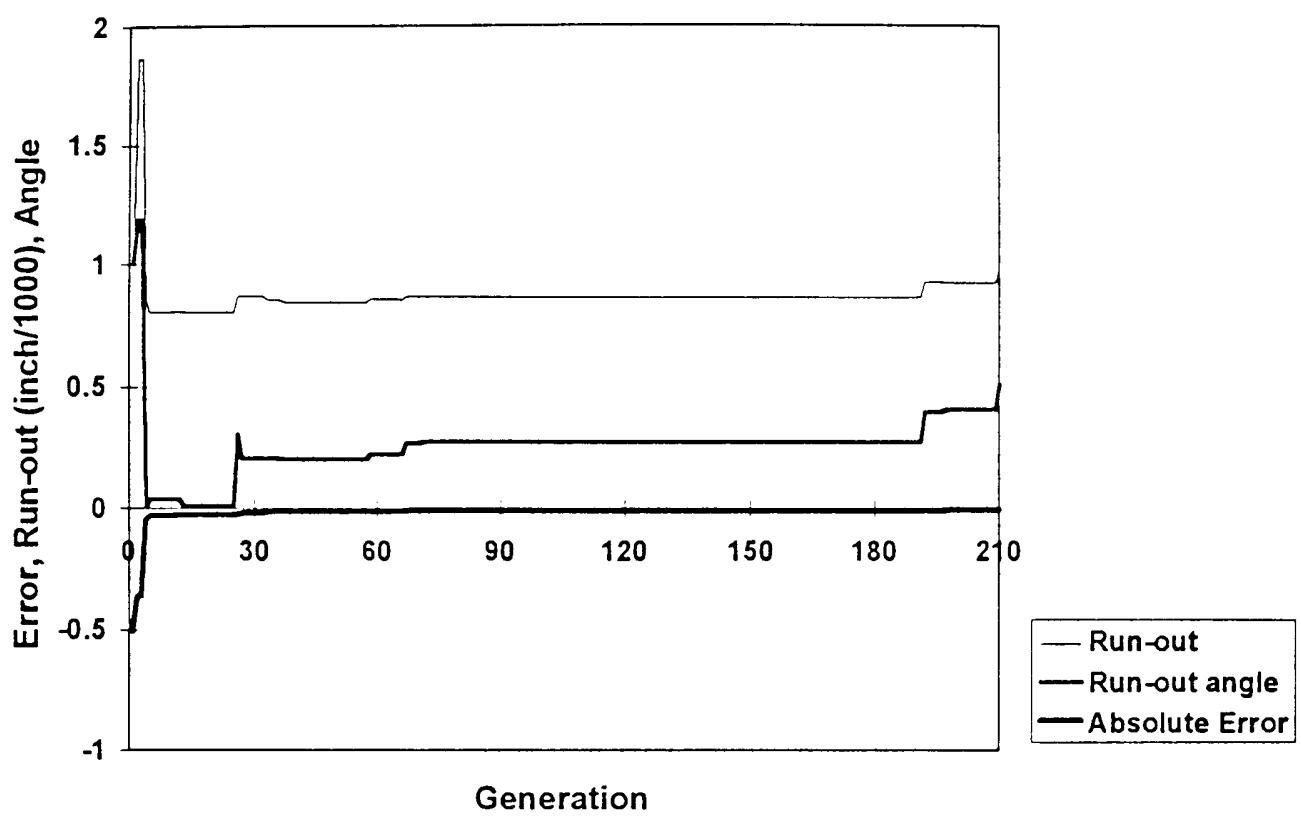

Figure 5.23 Genetic evolution procedure of tool run-out estimation

The accuracy and efficiency of the genetic algorithms were found satisfactory for this application. Accuracy and the computational time were related in reverse. It is found that $0.5 \%$ to $1 \%$ absolute error level is enough to obtain the required accuracy in very short time for most of the cases. 


\subsection{Cutting Angle Monitoring ${ }^{[19]}$}

The tool cutting angle of micro-end-milling is one of the interesting cutting conditions to be monitored. It is difficult to evaluate the sensory data to estimate the tool cutting angle because of the small tool diameter and continuous changed cutting angle in complicated machining profiles. Considering cutting force characteristics, it is proposed to compare the profile of the on-line recorded cutting force signals with one of the analytical estimated cutting force to identify the tool cutting angles. If the tool working conditions are unchanged in the end milling operations, the cutting force profile is only depended on the cutting angles.

The tool cutting angles are considered as entry and exit angles, which include all the cases of the climbing and conventional end milling operations. Two cases, $50 \%$ overlapping conventional and climbing milling, are presented in Figure 5.24.

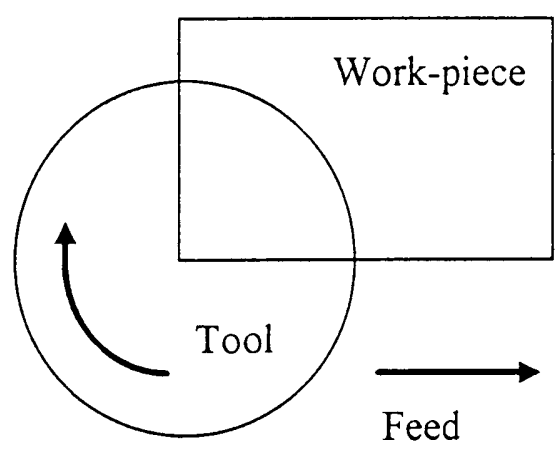

Case 1: Entry angle: 0 degree Exit angle: 90 degree

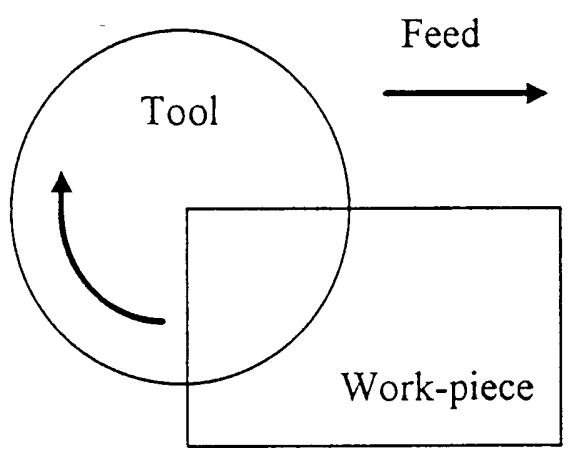

Case 2: Entry angle: 90 degree Exit angle: 180 degree

Figure 5.24 Tool cutting angles of end milling operations 
The profile of tool cutting force in the either feed or thrust direction can be used to identify the tool cutting angle. In the research, the resultant cutting force profile was chosen. An optimal objective function was introduced into the identification of tool cutting force profile. It is:

$$
\operatorname{Min}(E)=\sum_{i=1}^{N}\left[F_{\text {res }}^{\text {actual }}(i)-F_{\text {res }}^{\text {estimated }}(i)\right]^{2}
$$

where: $E$ is the square error.

$\mathrm{N}$ is the number of computing points in a tool turning cycle (360 degree).

$F_{\text {res }}^{\text {actual }}(i)$ and $F_{\text {res }}^{\text {estimated }}(i)$ are the monitoring and estimated resultant cutting forces in the $\mathrm{i}^{\text {th }}$ time or tool turning angle.

The genetic algorithm (see Chapter II) was applied to minimize the error of the optimal objective function. In the genetic evolution procedures, the 30 bits binary coding was used for individuals that include two factors, tool cutting entry and exit angle, with 15 bits each. The population size was selected as five, mating pool size as two versus two and one child from each couple. The uniform crossover (with 0.5 probability), jumping mutation (with 0.02 probability), creeping mutation (with 0.04 probability) and elitism were chosen.

The procedure of the tool cutting angle identification is presented in Figure 5.25. The tool cutting angles were estimated automatically according to the given tool geometry, cutting conditions, actual and estimating cutting force profiles. The actual and estimated maximum cutting forces were able to be easily obtained from the on-line recorded data and the analytical cutting force model (see Chapter III). The estimated tool 
cutting angles were improved generation by generation during the genetic evolution procedure until reach the optimal one.

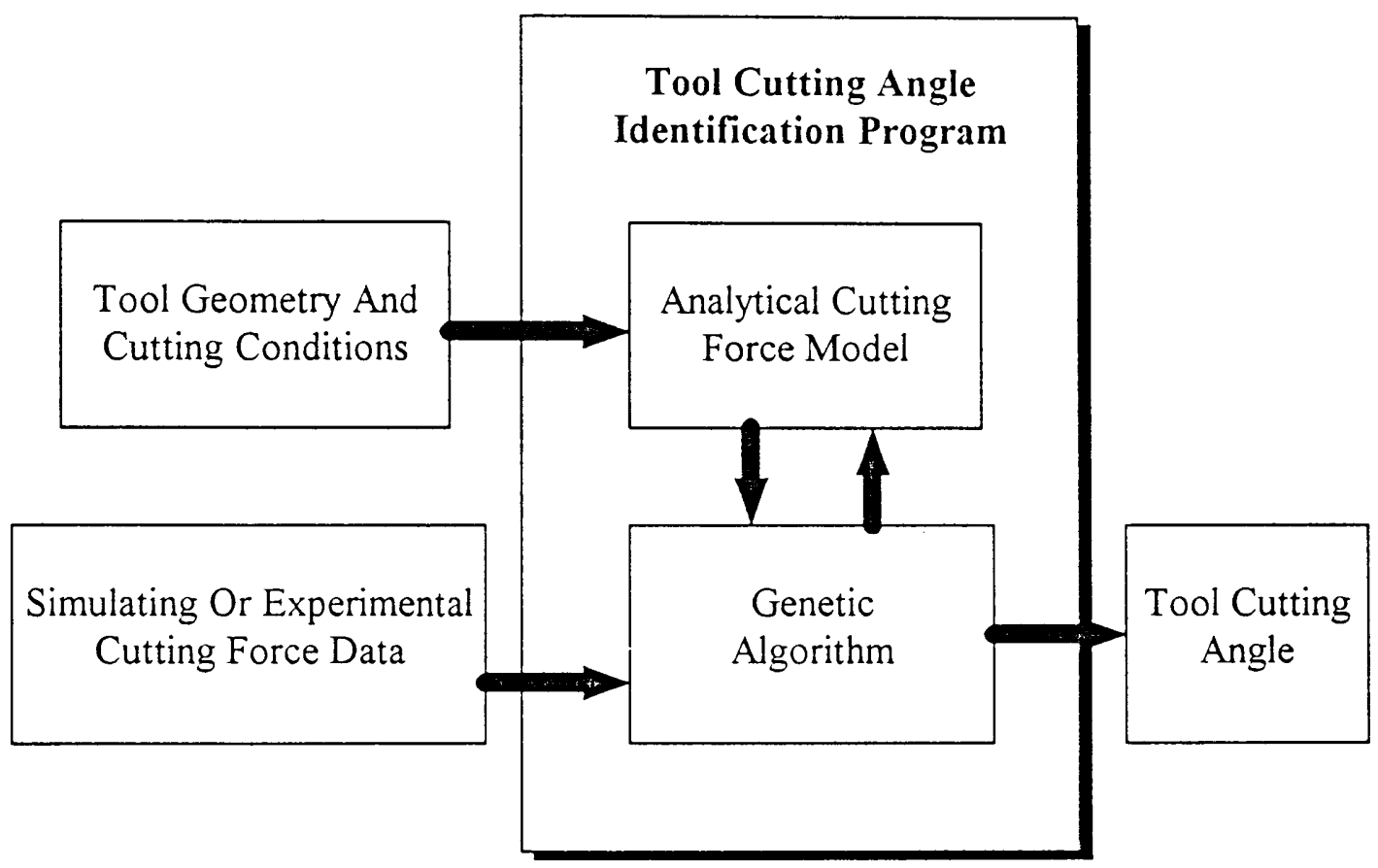

Figure 5.25 Tool cutting angle identification procedure

Four simulation cases were selected to test the performance of the tool cutting angle identification program. Two-flutes 0.020 " diameter tool with 45 degree helix angle, $15,000 \mathrm{rpm}$ spindle speed, $120 \mathrm{imp}$ feed rate, $0.010 \mathrm{inch}$ depth of cut and 80,000 N/inch ${ }^{2}$ material coefficient were selected in the micro-end-milling operations.

Case 1: $0^{\circ}$ entry angle and $90^{\circ}$ exit angle (50\% overlapping conventional milling).

Case 2: $90^{\circ}$ entry angle and $180^{\circ}$ exit angle (50\% overlapping climbing milling).

Case 3: $135^{\circ}$ entry angle and $180^{\circ}$ exit angle ( $85 \%$ overlapping climbing milling).

Case 4: $45^{\circ}$ entry angle and $135^{\circ}$ exit angle.

The results of four testing cases are presented in Table 5.2. 


\begin{tabular}{|c|c|c|c|c|c|c|}
\hline & & $\begin{array}{c}\text { Actual } \\
\text { Value }\end{array}$ & $\begin{array}{c}\text { Estimating } \\
\text { Value in 20th } \\
\text { Generation }\end{array}$ & $\begin{array}{c}\text { Error } \\
\text { in 20th } \\
\text { Generation }\end{array}$ & $\begin{array}{c}\text { Estimating } \\
\text { Value in 120th } \\
\text { Generation }\end{array}$ & $\begin{array}{c}\text { Error } \\
\text { in 120th } \\
\text { Generation }\end{array}$ \\
\hline \multirow{4}{*}{ Case 1 } & Entry Angle & 0 & 0.170 & 0.027 & 0.001 & 0.000 \\
\cline { 2 - 8 } & Exit Angle & 1.571 & 1.592 & 0.003 & 1.571 & 0.000 \\
\cline { 2 - 8 } & Fitness & 0 & -0.036 & 0.036 & 0.000 & 0.000 \\
\hline \multirow{4}{*}{ Case 2 } & Entry Angle & 1.571 & 1.404 & 0.027 & 1.545 & 0.004 \\
\cline { 2 - 8 } & Exit Angle & 3.142 & 3.129 & 0.002 & 3.142 & 0.000 \\
\cline { 2 - 8 } & Fitness & 0 & -4.732 & 4.732 & 0.000 & 0.000 \\
\hline \multirow{4}{*}{ Case 3 } & Entry Angle & 2.356 & 2.377 & 0.003 & 2.400 & 0.007 \\
\cline { 2 - 8 } & Exit Angle & 3.142 & 3.129 & 0.002 & 3.142 & 0.000 \\
\cline { 2 - 8 } & Fitness & 0 & 0.000 & 0.000 & 0.000 & 0.000 \\
\hline \multirow{3}{*}{ Ease 4 } & Entry Angle & 0.785 & 0.847 & 0.010 & 0.835 & 0.008 \\
\cline { 2 - 8 } & Exit Angle & 2.356 & 2.254 & 0.016 & 2.353 & 0.000 \\
\cline { 2 - 8 } & Fitness & 0 & -0.663 & 0.663 & -0.021 & 0.021 \\
\hline
\end{tabular}

Table 5.2 Results of the tool cutting angle identification

In all of the studied cases, the tool entry and exit cutting angles were estimated in less than 20 generations with less than $3 \%$ error and in 120 generations with less than $1 \%$ error.

The identification program has a good performance by using genetic algorithm. It has fast reactions and accurate results for the on-line monitoring. The more high sampling resolution is selected, the more accuracy results will be obtained. It will take a little more running time to find a qualified generation. It is found that 72 points per tool turning cycle are enough to obtain the required accuracy with the fast reaction in many testing cases. 


\subsection{Cutting Condition Monitoring}

The most interesting cutting conditions are spindle speed, feed rate and depth of cut. They can be obtained by monitoring the cutting force signals in micro-end-mill operations.

The spindle speed of micro-end-mill operations can be directly obtained from the cutting force signals. The method to monitor the spindle speed is to take a cutting force value from the cutting force signal and find the same cutting force value after one cutting cycle (see Figure 5.26). The interval time $\mathrm{T}$ between these two points can be used to calculate the spindle speed by formula 5.5.1.

$$
n=\frac{60}{T}
$$

\section{Thrust Direction Cutting Force of Climbing End- Milling with Tool Run-out}

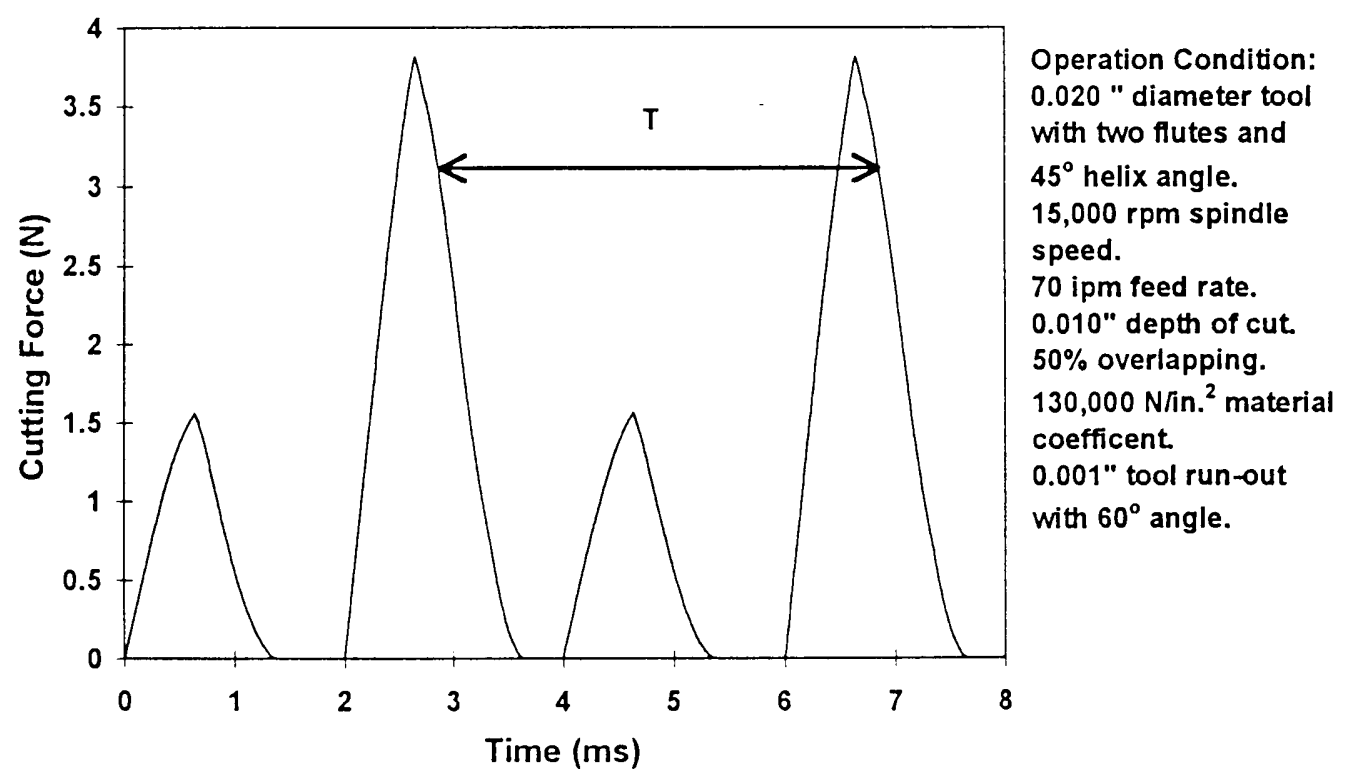

Figure 5.26 Spindle speed identification 
The feed rate and depth of cut can be obtained by using the same method as monitoring cutting angle, which is to compare the profile of the on-line recorded cutting force with one of the analytical estimated cutting force. The only difference is to use feed rate and depth of cut instead of entry and exit cutting angles. The genetic algorithm also can be applied to find the fittest feed rate and depth of cut. The optimal objective function has the same form as the formula 5.3.1. The procedure of the method is presented in Figure 5.27. The feed rate and depth of cut can be estimated automatically according to the given tool geometry, cutting angles, actual and estimating cutting force profiles. The actual and estimated cutting force profiles can be easily obtained from the on-line cutting force recording and the analytical cutting force model (see Chapter III).

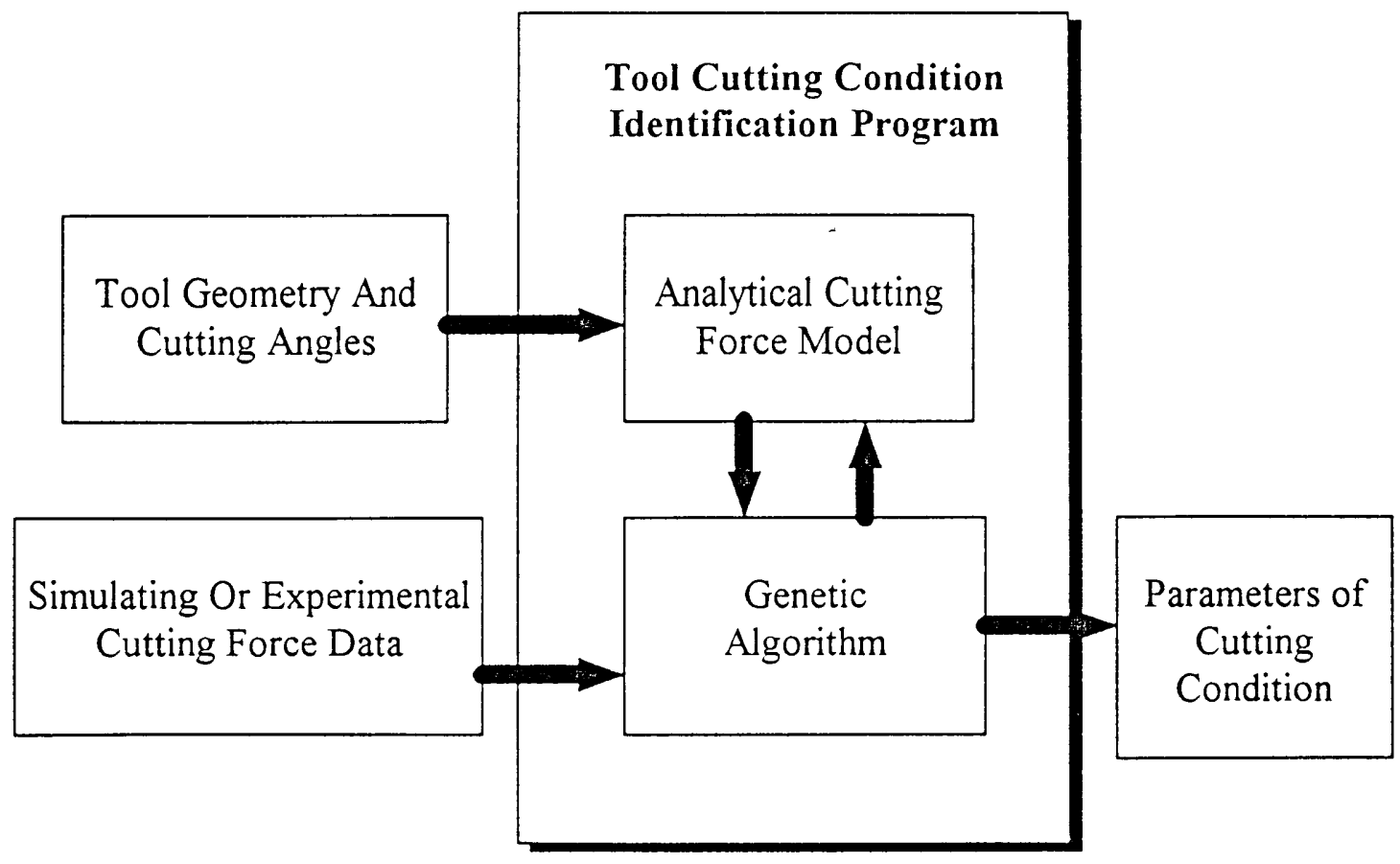

Figure 5.27 Tool cutting condition identification procedure 


\subsection{Optimal Working Condition Selection}

In micro-end-milling operations, it is important to select the tool optimal working conditions. Because the tiny tools are very easily broken, the conservative selections of the tool working conditions would cost longer machining time, otherwise the unsuitable selections of the tool working conditions would make change the tools frequently that wastes the machining time too. It is very difficult for operators to select the optimal working conditions in so many different types of tools, work-pieces and different machining tasks.

In the research, the tool working conditions were optimized based on the minimum machining time, that is to find the maximum feed rate that is able to meet the tool life requirement in the specific machining task. It is known how many cutting inches are required in the specific machining task. Approximated tool life can be estimated depended on how many tools will be used in the task. Referred to tool life estimation (5.2), the maximum feed rate can be determined with the other possible working conditions (for example, maximum spindle speed of the machine tool) by the analytical cutting force model.

The genetic algorithms were used to optimize the tool working conditions with the analytical cutting force model. The maximum cutting forces of the cutting force profiles, which were got from the analytical model and estimation, were used for the optimal objective function and conditions as the following.

Objective function:

$$
\operatorname{Min}(E)=\frac{1}{2}\left(\left|F_{X \max }^{\text {estimated }}-F_{X \max }^{\text {allowable }}\right|+\left|F_{Y \max }^{\text {estimated }}-F_{Y \max }^{\text {allowable }}\right|\right)
$$

Conditions:

$$
\begin{aligned}
& F_{X \max }^{\text {estimated }} \leq F_{X \max }^{\text {allowable }} \\
& F_{Y \max }^{\text {estimated }} \leq F_{Y \max }^{\text {allowable }}
\end{aligned}
$$

where: $E$ is the absolute error. 
$F_{X \max }^{\text {allowable }}$ and $F_{Y \max }^{\text {allowable }}$ are the allowable maximum cutting forces in feed and thrust directions, which can be determined by the tool life estimation.

$F_{X \max }^{\text {estimated }}$ and $F_{Y \max }^{\text {estimated }}$ are the estimated maximum cutting forces in feed and thrust directions, which can be calculated by the analytical cutting force model.

The tool working condition optimization program is included in the MOGART program (see Chapter VIII). In the program, the tool working conditions can be selected automatically according to the required tool life. The procedure of the tool working condition optimization is presented in Figure 5.28. The program user interface is presented in Figure 8.24 of Chapter VIII.

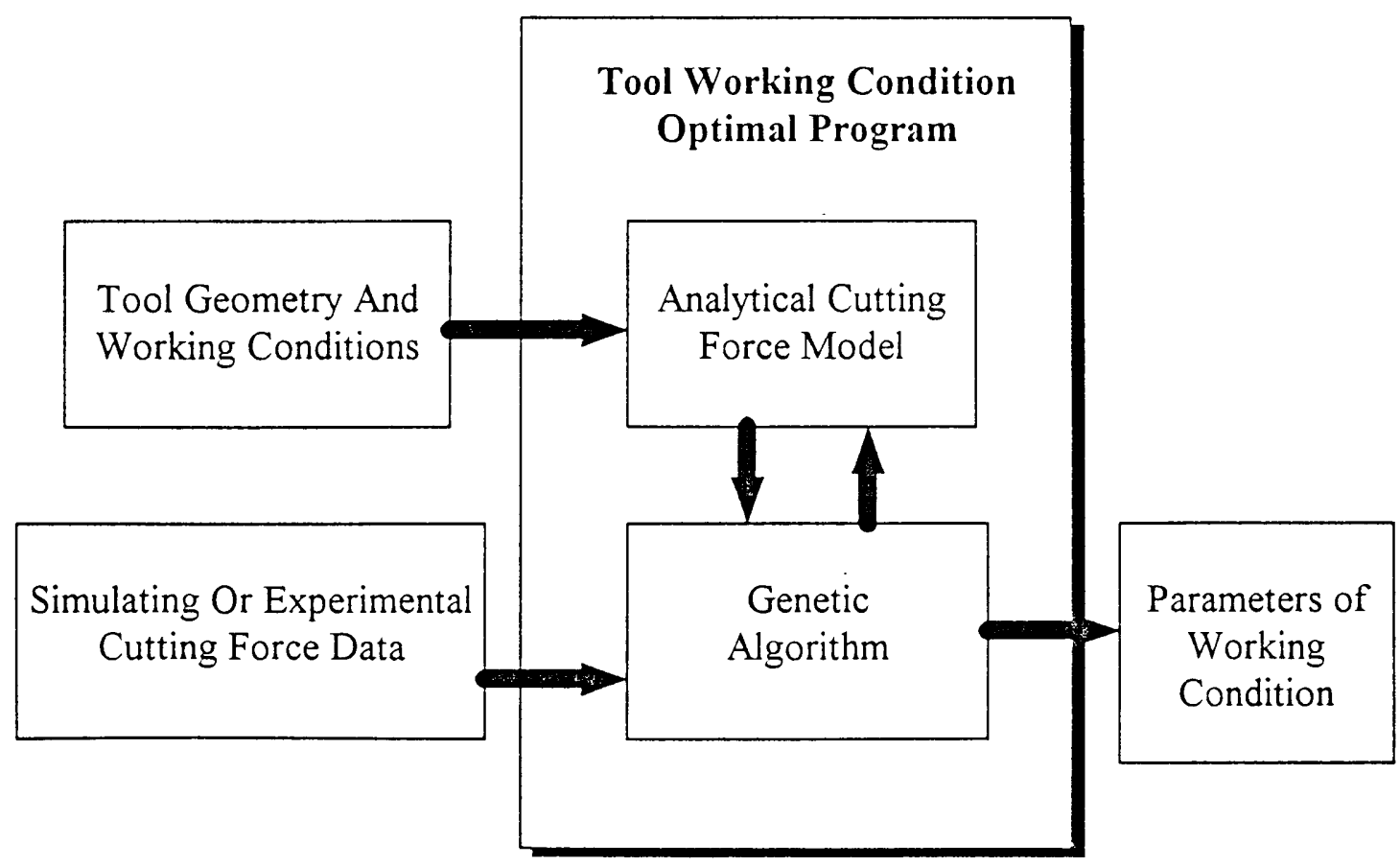

Figure 5.28 Tool optimal working condition estimation procedure 


\section{CHAPTER VI}

\section{Experiment Setup}

All the experiments were performed at Mechatronics Lab, Mechanical Engineering Department, Florida International University and Engineering Prototype Center, Radio Technology Division, Motorola Inc. More than 800 cutting experiments were performed and more than 160 megabytes of cutting force data were recorded. The experimental contents are listed in Table 6.1.

A typical experimental setup is presented in Figure 6.1. Three different milling machines were used in the experiments. The work-piece to be tested was installed on a dynamometer, which was clamped on the table of the machine tool. Two components of the cutting forces were recorded by using a digital oscilloscope through a charge amplifier. All the experimental equipment is listed in Table 6.2 and shown in Figures 6.2 to 6.7.

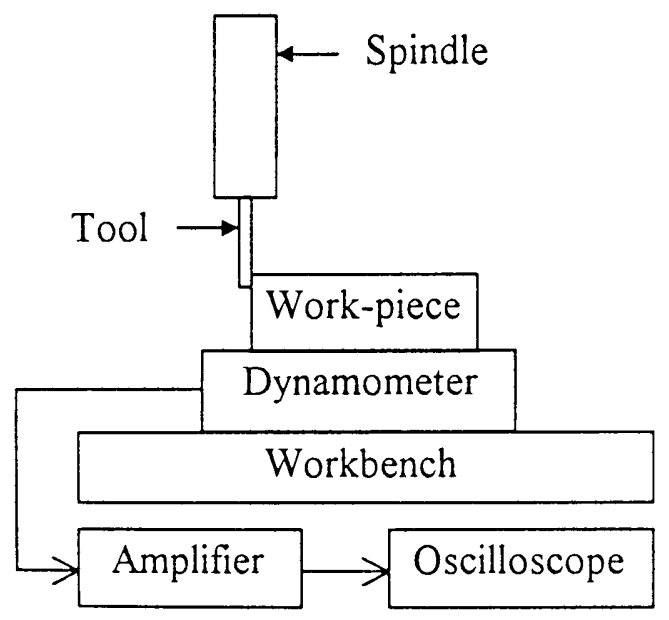

Figure 6.1 Experimental setup 


\begin{tabular}{|l|l|}
\hline Tool type: & Two-flute and four-flute micro-end-mill \\
\hline Tool diameter (inch): & $15 / 1000,20 / 1000,30 / 1000,1 / 32,1 / 16$ and $1 / 8$ \\
\hline Tool material: & High speed steel (HSS) and carbide \\
\hline Work-piece material: & $\begin{array}{l}\text { Aluminum, copper and mild steel } \\
\text { Steel: NAK-55, P-20, 420, Moly, Aged Moly and 3DP } \\
\text { Non-metal: POCO-3 and POCO-C3 graphite. }\end{array}$ \\
\hline Cutting conditions: & Slot and 50\% overlapped climbing end milling with \\
different spindle speed, feed rate and depth of cut.
\end{tabular}

Table 6.1 Experimental contents

\begin{tabular}{|l|l|}
\hline Machine tool: & $\begin{array}{l}\text { Bridgeport series I 3,000 rpm milling machine } \\
\text { Fadal 3-axis 15,000 rpm CNC machine } \\
\text { Fadal 5-axis 50,000 rpm CNC machine }\end{array}$ \\
\hline Data acquisition: & $\begin{array}{l}\text { Nicolet 310 digital oscilloscope } \\
\text { Nicolet integra model 10 digital oscilloscope }\end{array}$ \\
\hline Cutting force measurement: & Kistler 9257B 3-component piezoelectric dynamometer \\
& Kistler 3-channel charge amplifier \\
\hline Displacement measurement: & Kaman KD2310-2S measuring systems \\
\hline Hardness measurement: & Wilson TU220 \\
\hline Image processing system: & Olympus SZH10 microscope and SONY DXC-107A \\
& digital color video camera \\
\hline
\end{tabular}

Table 6.2 Experimental equipment 


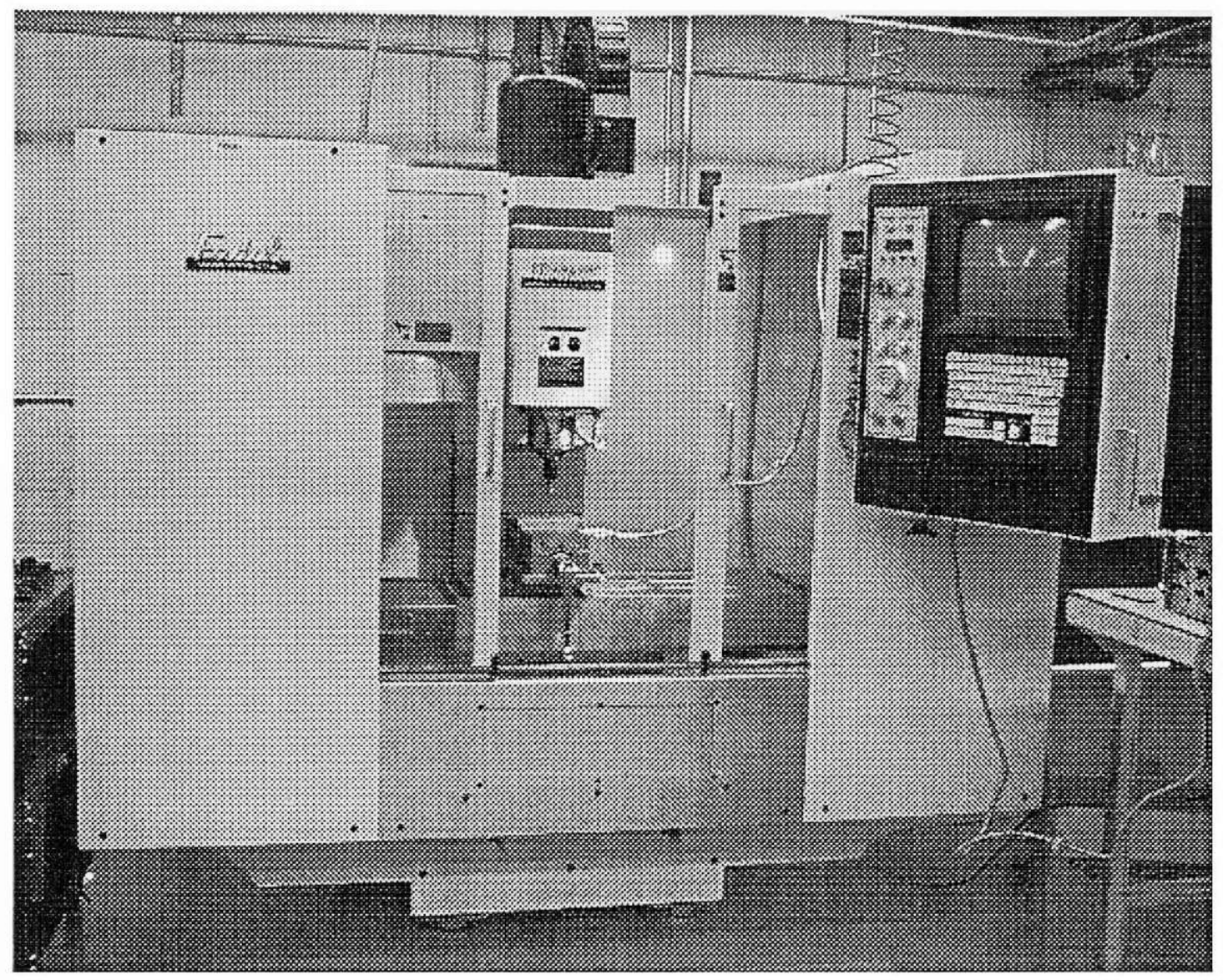

Figure 6.2 Machine tool

Majority of the experiments was performed on Fadal 5-axis 50,000 rpm CNC machine. Fadal 3-axis 15,000-rpm CNC machine. Bridgeport series I 3,000 rpm milling machine was also used in some experiments. 


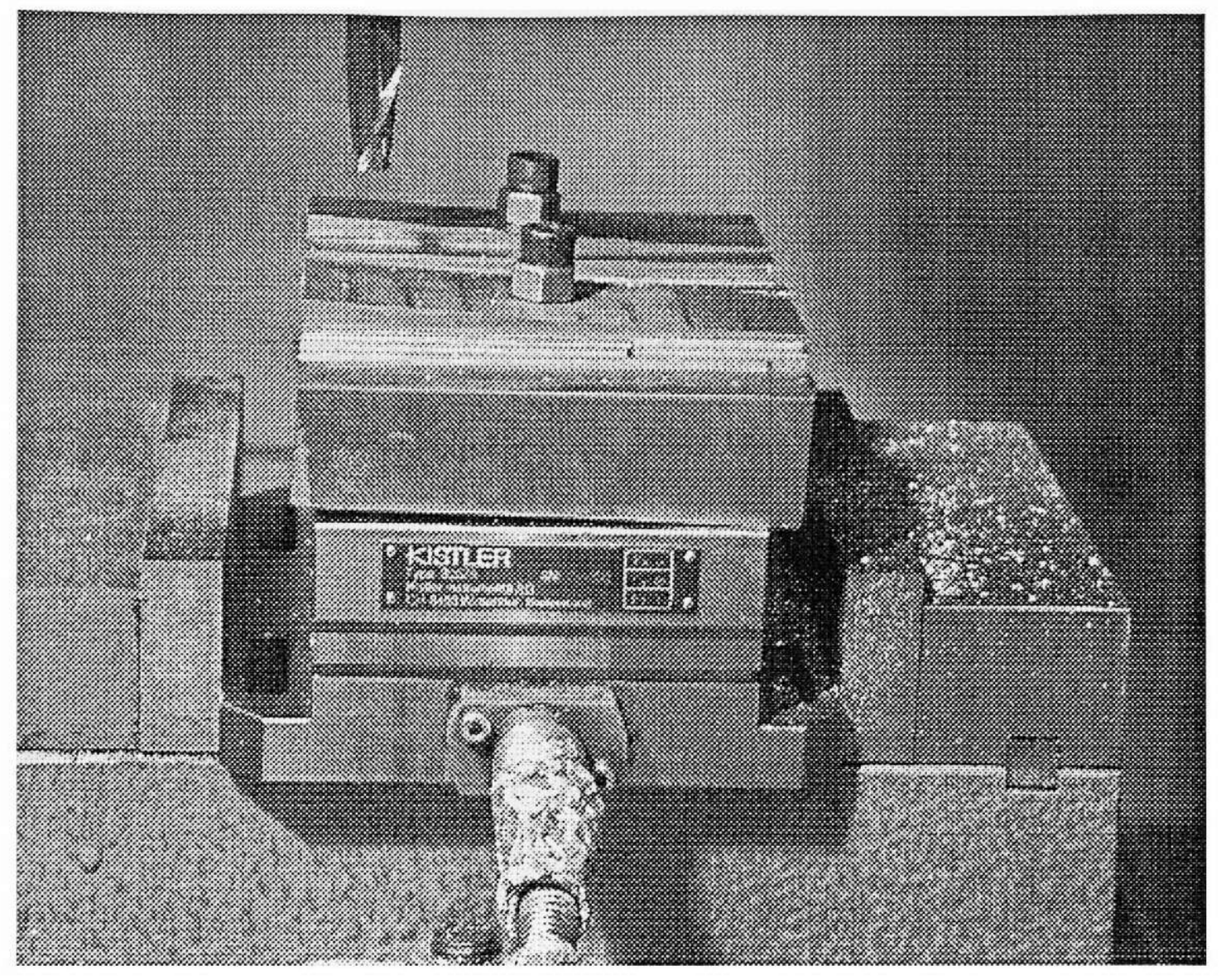

Figure 6.3 Cutting force measurement

Kistler 9257B 3-component piezoelectric dynamometer was used to monitor twodimension (feed direction and thrust direction) cutting forces. 


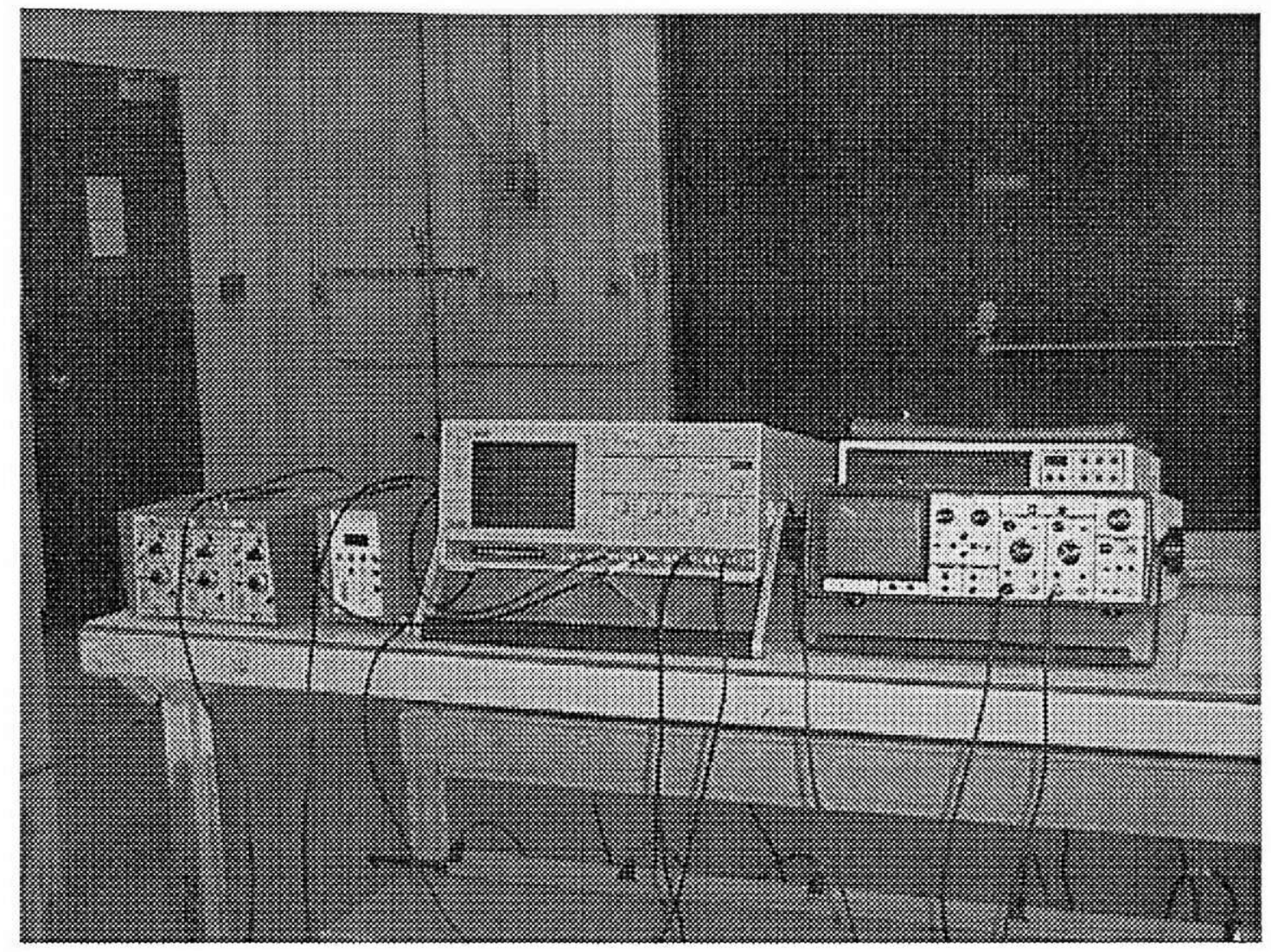

Figure 6.4 Data acquisition

Two digital oscilloscopes (Nicolet 310 digital oscilloscope and Nicolet integra model 10 digital oscilloscope) were used to record the cutting force and acoustic emission signals, which were amplified by using a Kistler 3-channel charge amplifier. 


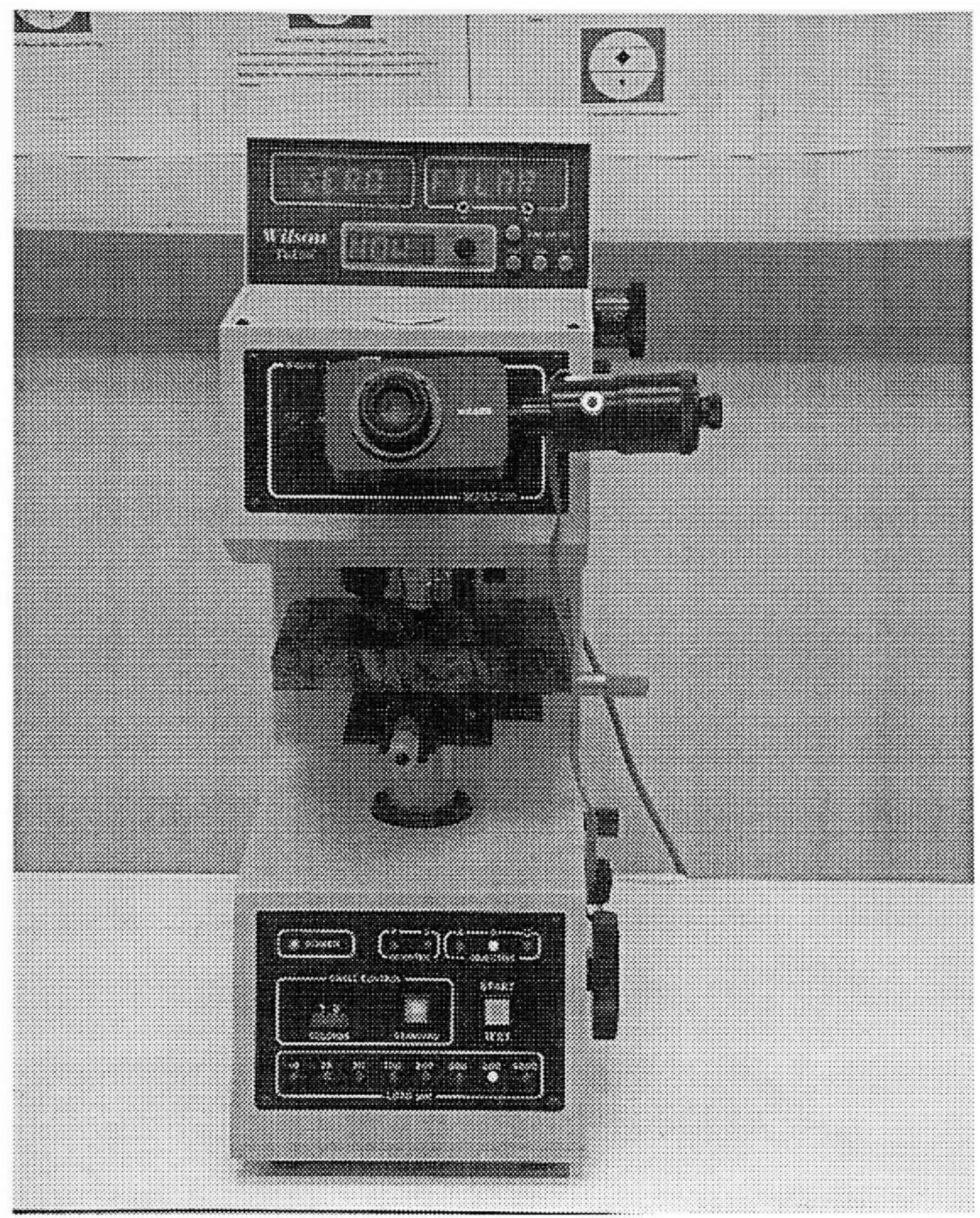

Figure 6.5 Hardness measurement

The Wilson TU220 hardness measurement machine was used to test the hardness of the work-pieces. 


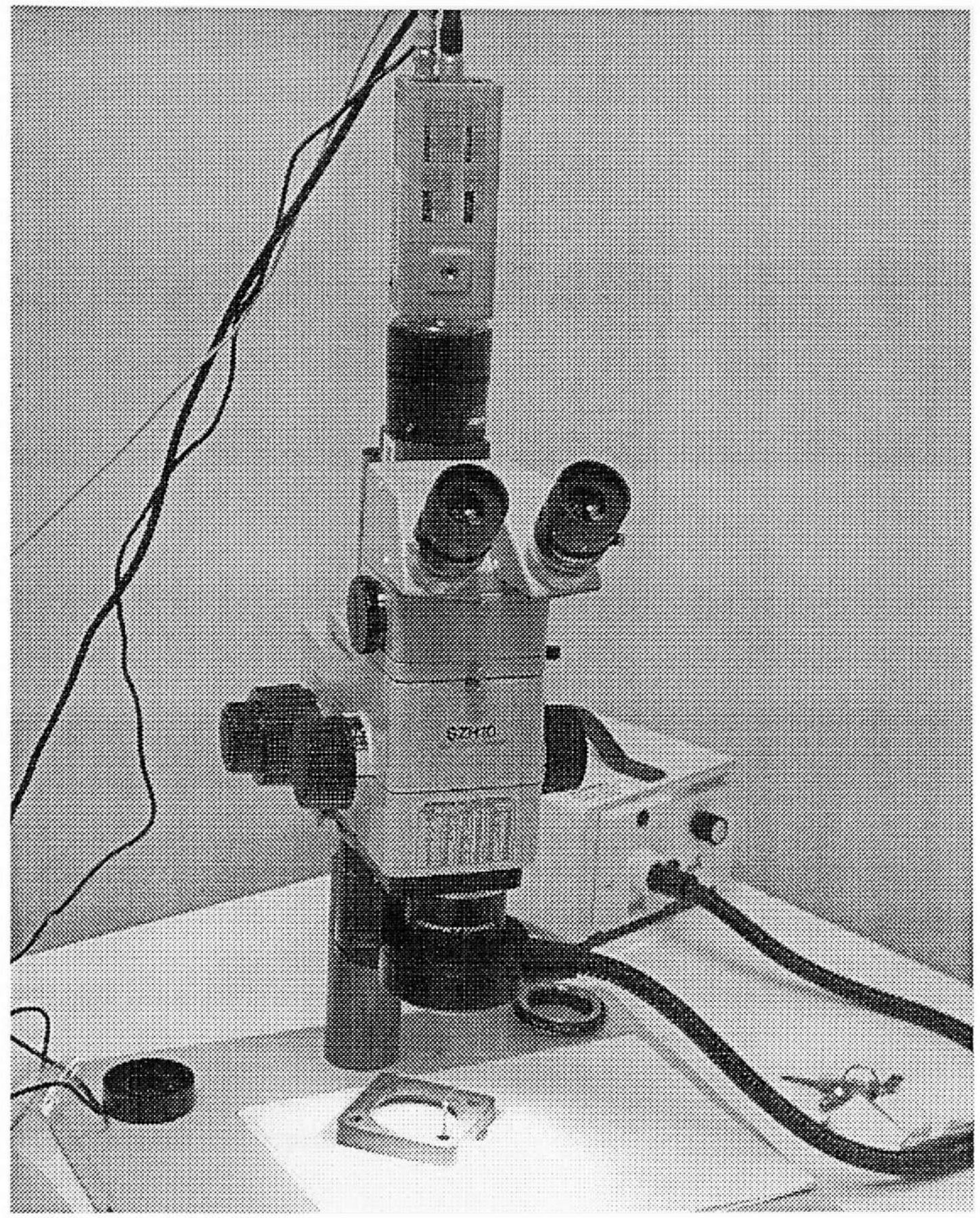

Figure 6.6 Image processing system

The Image processing system used Olympus SZH10 microscope and SONY DXC$107 \mathrm{~A}$ digital color video camera to investigate tool wear. 


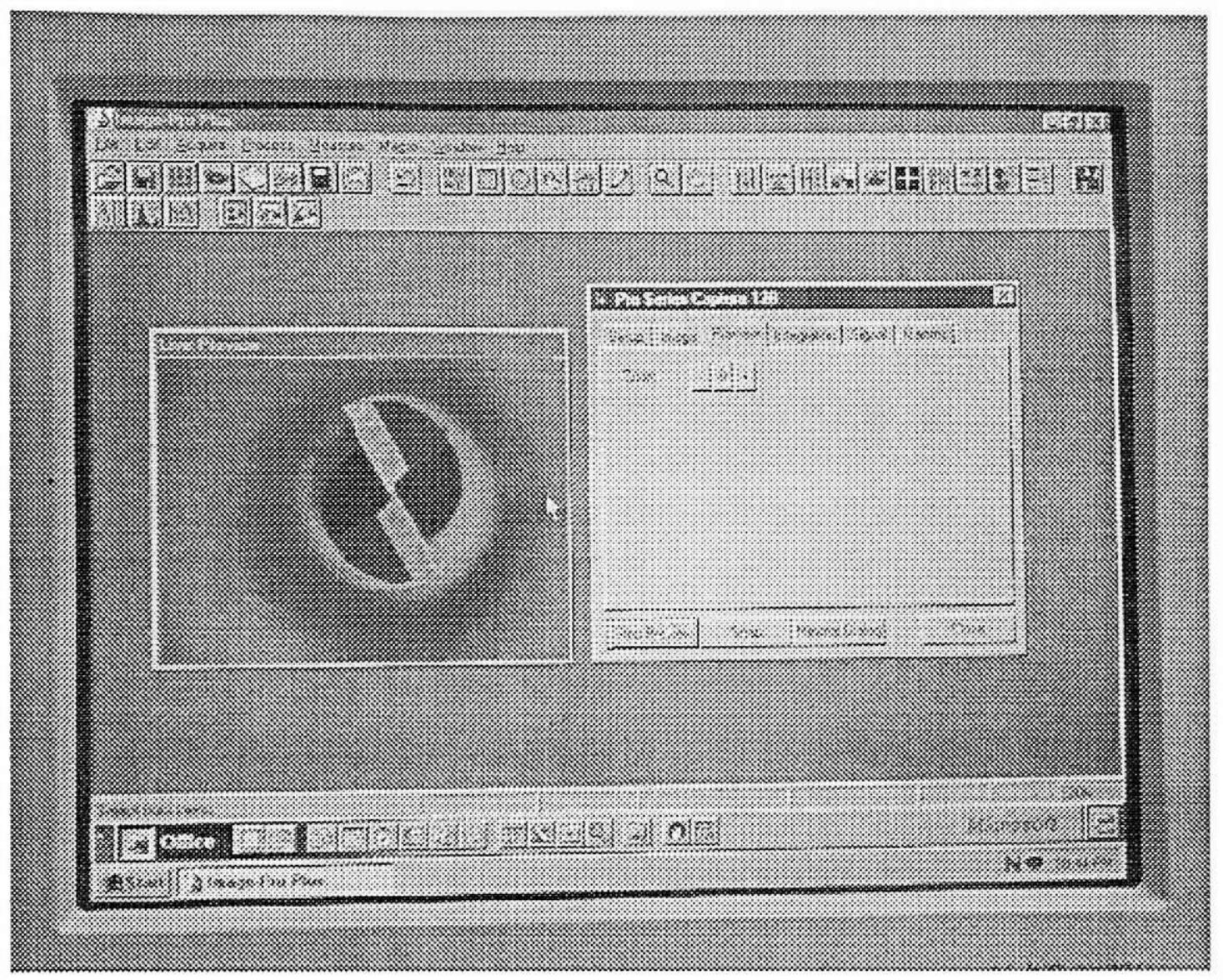

Figure 6.7 The computer screen showing the software used for Image processing

Figure 6.8 and 6.9 shows the cutting edges of a new tool and a worn-out tool respectively and the figure 6.10 and 6.11 shows the work-piece surfaces cut by a new tool and by a worn-out tool respectively. 


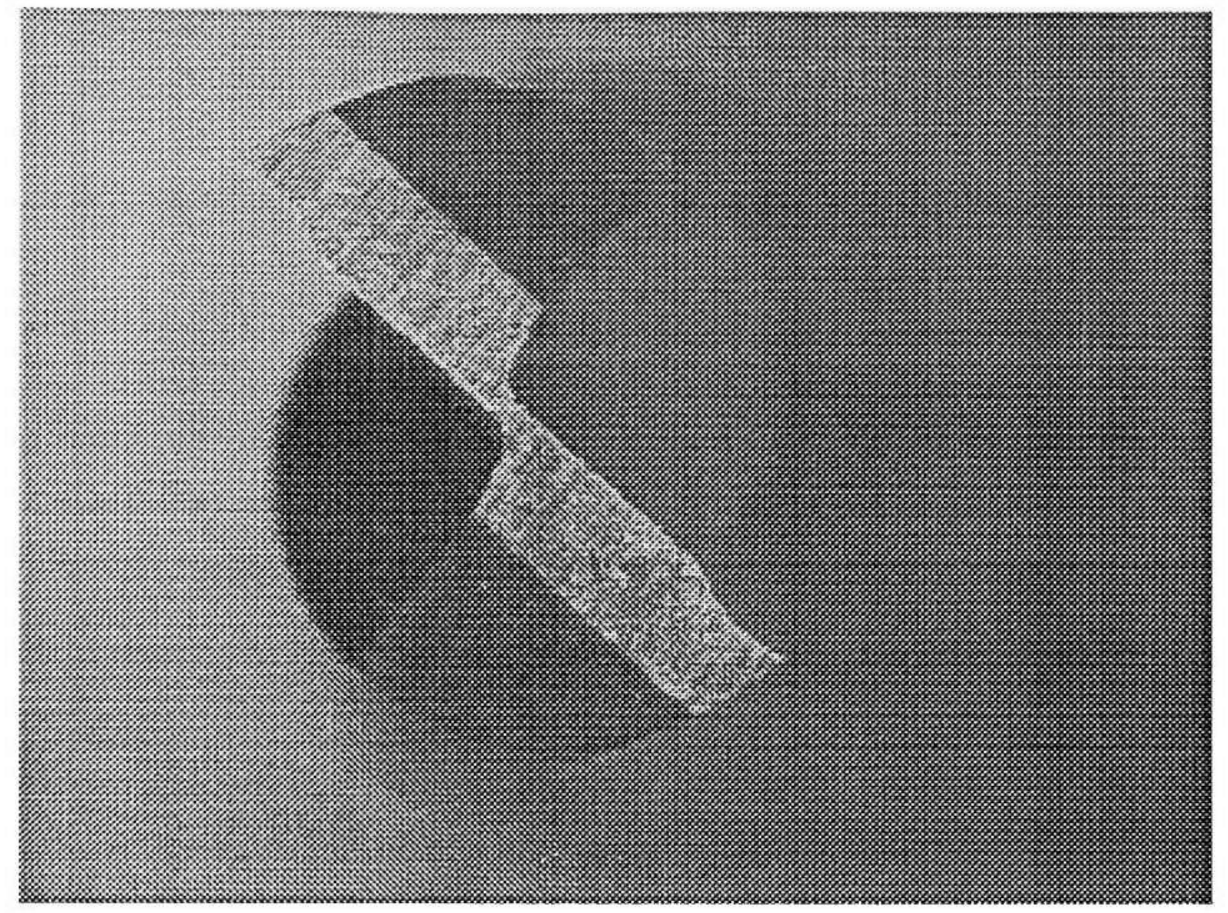

Figure 6.8 The cutting edges of a New tool

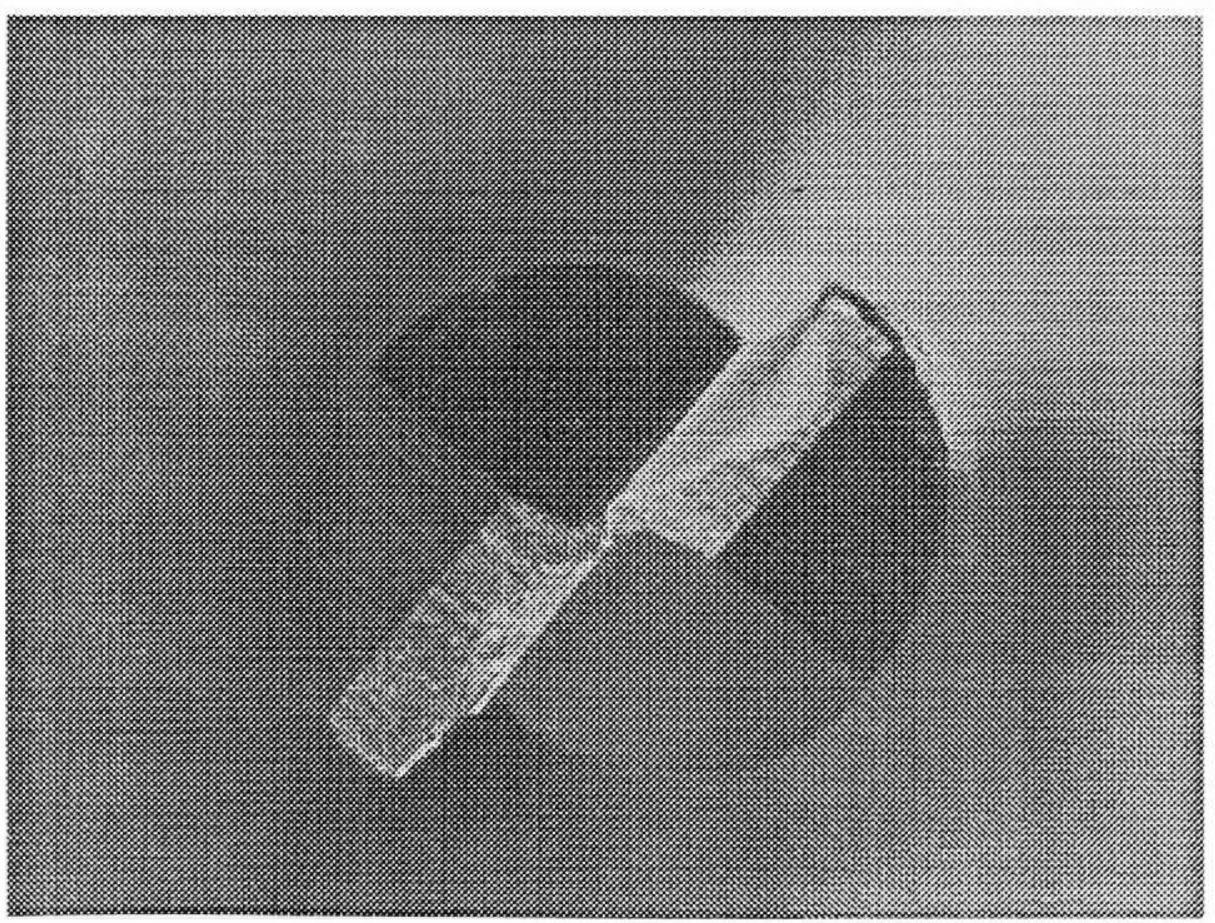

Figure 6.9 The cutting edges of a Worn tool 


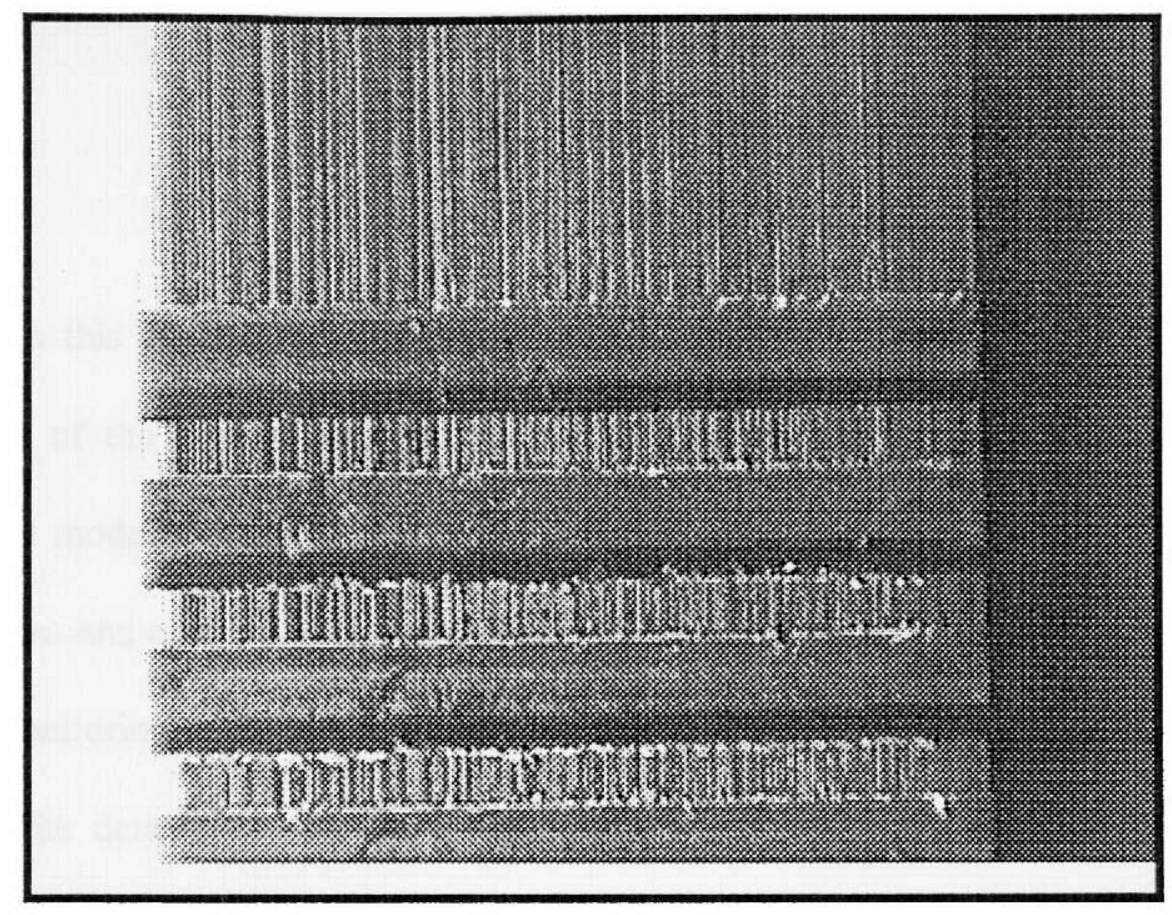

Figure 6.10 Work-piece surface cut by a new tool

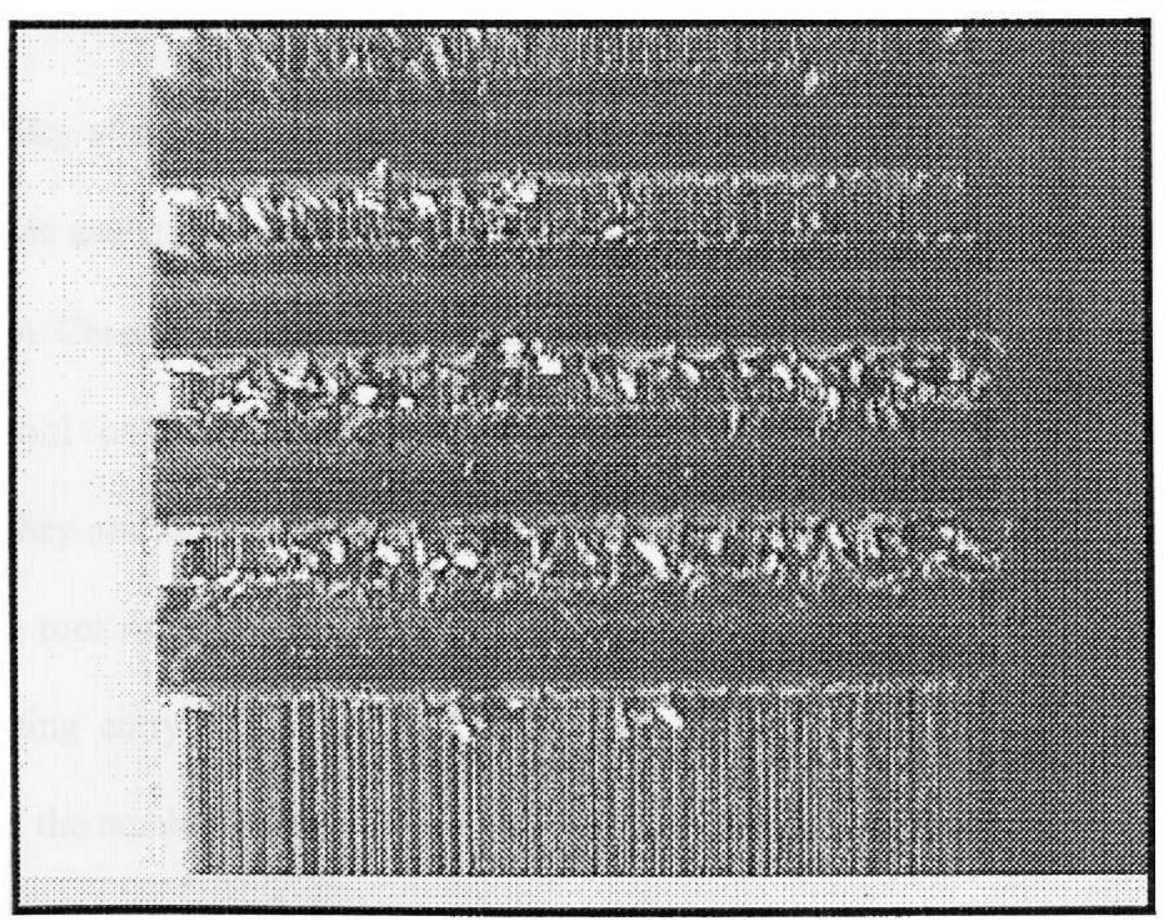

Figure 6.11 Work-piece surface cut by a worn tool 


\section{Chapter VII \\ Results and Discussion}

In this chapter, the validity of the proposed analytical model is discussed. The accuracy of the cutting force estimation of the two approaches (neural networks and analytical models) has been evaluated on the experimental data. The analytical models of both micro and conventional end milling have been compared. Also the genetic algorithms based monitoring techniques has been tested on the simulated and experimental data.

The data from more than 800 experimental cases was used to investigate the cutting force characteristics and some of the data was used to verify the validity of the developed techniques.

\subsection{Validity of the Analytical Cutting Force Model ${ }^{[19]}$}

The analytical cutting force model of the micro-end-mill machining operations was derived in Chapter III, which included the micro and conventional end milling with or without tool run-out and wear cases. Ten parameters and two coefficients are used in this model. They are three working condition variables (spindle speed, feed rate and depth of cut), two tool run-out variables (run-out and its angle), two cutting condition variables (tool cutting entry and exit angle), three tool geometry variables (tool diameter, helix angle and the numbers of tool flutes), material and wear coefficients.

The analytical cutting force model of end milling operations can been expressed in the following formulas: 
- The developed cutting force mode of end milling operations

$$
\begin{aligned}
& F_{x}=F_{u} {\left[C_{3} \frac{f_{t}}{r} \sin ^{3} \theta+C_{4} \frac{f_{t}}{r} \cos ^{3} \theta-\left(1+C_{5}\right) \sin ^{2} \theta+\frac{1}{2} p\left(1+C_{5}\right) \sin 2 \theta\right.} \\
&\left.+\left(C_{6}-\frac{f_{t}}{r}\right) \sin \theta-p C_{6} \cos \theta-p\left(1+C_{5}\right) \theta\right]\left.\right|_{\theta_{s}} ^{\theta_{e}} \\
& F_{y}=F_{u}\left[C_{4} \frac{f_{t}}{r} \sin ^{3} \theta-C_{3} \frac{f_{t}}{r} \cos ^{3} \theta-p\left(1+C_{5}\right) \sin ^{2} \theta-\frac{1}{2}\left(1+C_{5}\right) \sin 2 \theta\right. \\
&\left.+p\left(C_{6}-\frac{f_{t}}{r}\right) \sin \theta+C_{6} \cos \theta+\left(1+C_{5}\right) \theta\right]\left.\right|_{\theta_{s}} ^{\theta_{s}}
\end{aligned}
$$

- Micro-end milling operations without tool run-out

$$
\begin{aligned}
& F_{x}=\left.F_{u}\left[C_{1} \frac{f_{t}}{r} \sin ^{3} \theta+C_{2} \frac{f_{t}}{r} \cos ^{3} \theta-\sin ^{2} \theta+\frac{1}{2} p \sin 2 \theta-\frac{f_{t}}{r} \sin \theta-p \theta\right]\right|_{\theta_{s}} ^{\theta_{e}} \\
& F_{y}=\left.F_{u}\left[C_{2} \frac{f_{t}}{r} \sin ^{3} \theta-C_{1} \frac{f_{t}}{r} \cos ^{3} \theta-p \sin ^{2} \theta-\frac{1}{2} \sin 2 \theta-p \frac{f_{t}}{r} \sin \theta+\theta\right]\right|_{\theta_{s}} ^{\theta_{e}}
\end{aligned}
$$

- Conventional end milling operations with tool run-out

$$
\begin{aligned}
& F_{x}=\left.F_{u}\left[-\sin ^{2} \theta+\frac{1}{2} p \sin 2 \theta \pm \frac{4 r_{o}}{f_{t}} \cos \gamma(\sin \theta-p \cos \theta)-p \theta\right]\right|_{\theta_{s}} ^{\theta_{e}} \\
& F_{y}=\left.F_{u}\left[-p \sin ^{2} \theta-\frac{1}{2} \sin 2 \theta \pm \frac{4 r_{o}}{f_{t}} \cos \gamma(p \sin \theta+\cos \theta)+\theta\right]\right|_{\theta_{s}} ^{\theta_{s}}
\end{aligned}
$$

- Conventional end milling operations without tool run-out

$$
\begin{aligned}
& F_{x}=\left.F_{u}\left[-\sin ^{2} \theta+\frac{1}{2} p \sin 2 \theta-p \theta\right]\right|_{\theta_{s}} ^{\theta_{e}} \\
& F_{y}=\left.F_{u}\left[-p \sin ^{2} \theta-\frac{1}{2} \sin 2 \theta+\theta\right]\right|_{\theta_{s}} ^{\theta_{s}} \\
& \text { where }: F_{u}=\frac{K_{w} K_{m} r f_{t}}{2 \tan \beta}
\end{aligned}
$$




$$
\begin{array}{ll}
C_{1}=\frac{1}{3}\left(1+p \frac{Z}{\pi}\right) & C_{2}=\frac{1}{3}\left(p-\frac{Z}{\pi}\right) \\
C_{3}=\frac{1}{3}\left(1+p \frac{2}{\pi}\right) & C_{4}=\frac{1}{3}\left(p-\frac{2}{\pi}\right) \\
C_{5}= \pm \frac{2 r_{o}}{\pi r} \sin \gamma & C_{6}= \pm \frac{4 r_{o}}{f_{t}} \cos \gamma
\end{array}
$$

The formulas 7.1.1 and 7.1.2 are the basic formulas of the developed cutting force model of the end milling operations. The others can be simply derived from them. For example, formulas 7.1.3 and 7.1.4 can be derived from the formulas 7.1.1 and 7.1.2 by considering tool run-out $r_{0}=0$. In the conventional end milling operation case, formulas 7.1.5, 7.1.6 and 7.1.7, 7.1.8 can be derived from the formulas 7.1.1 and 7.1.2 by considering $f_{t} / r=0$, and both $r_{0}=0$ and $f_{t} / r=0$ respectively. In the conventional end milling without tool run-out, the formulas 7.1.7 and 7.1.8 exactly match Tlusty's model.

The develop analytical cutting force model has been tested on many experimental cases of the micro-end-milling operations with different tool, work-piece and cutting condition. Very good agreement has been observed between the theoretical and experimental results.

The cutting forces of one experimental case of micro-end-mill operation without tool run-out are presented in Figure 7.1. Two-flute 1/8" diameter carbide end-mill and steel work-piece were tested in the experiment. The working conditions were $2,000 \mathrm{rpm}$ spindle speed, $1 \mathrm{ipm}$ feed rate, 0.0625 " depth of cut and $50 \%$ overlapped climbing end milling. At the same cutting conditions, the cutting forces calculated by using the analytical cutting force model are presented in Figure 7.2. The maximum cutting force error of both the cutting forces was less than $1 \%$, which is presented in Table 7.1. 
The cutting forces of one experimental case of micro-end-mill operations with tool run-out are presented in Figure 7.3. Two-flute 1/16" diameter high speed steel end mill and POCO-3 graphite work-piece were tested in the experiment. The working conditions were $15,000 \mathrm{rpm}$ spindle speed, $100 \mathrm{ipm}$ feed rate, 0.0625 " depth of cut and $50 \%$ overlapped climbing end milling. The tool run-out was 0.001 " with 50 degree angle. At the same cutting conditions, the cutting forces calculated by using the analytical cutting force model are presented in Figure 7.4. The maximum cutting force error of both the cutting forces was with less than $3 \%$, which is presented in Table 7.1.

\begin{tabular}{|c|c|c|c|c|c|}
\hline & Flute & Direction & \multicolumn{2}{|c|}{ Maximum cutting force } & \multirow{2}{*}{ Error } \\
\cline { 3 - 5 } & & & Experiment & Model Based & \\
\hline \multirow{2}{*}{$\begin{array}{l}\text { Without tool } \\
\text { run-out case }\end{array}$} & Both & Thrust & 87.3 & 87.82 & $0.60 \%$ \\
\cline { 3 - 5 } & & Feed & 54.6 & 54.73 & $0.15 \%$ \\
\hline \multirow{3}{*}{$\begin{array}{l}\text { With tool } \\
\text { run-out case }\end{array}$} & 1 st & Thrust & 9.07 & 7.99 & $2.51 \%$ \\
\cline { 3 - 5 } & & Feed & 43.07 & 43.13 & $0.14 \%$ \\
\cline { 3 - 5 } & 2nd & Thrust & 6.25 & 6.14 & $0.26 \%$ \\
\cline { 3 - 5 } & & Feed & 17.62 & 17.54 & $0.19 \%$ \\
\hline
\end{tabular}

Table 7.1 Maximum cutting force error of the analytical cutting force model

In the above two presented cases, the material coefficients are $68,000,000$ and $115,000 \mathrm{~N} /$ inch $^{2}$ with 1.78 and 5.85 feed rate correction respectively. New tools were considered in both the cases so that tool wear coefficient is 1 .

Since the analytical cutting force model was developed based on two general assumptions, it could be considered as a theoretical foundation for end milling operations including both micro and conventional end milling operations. 
Cutting Forces of Micro-End-Milling Operations

2 flute $1 / 8$ " diameter carbide end mill, steel work-piece

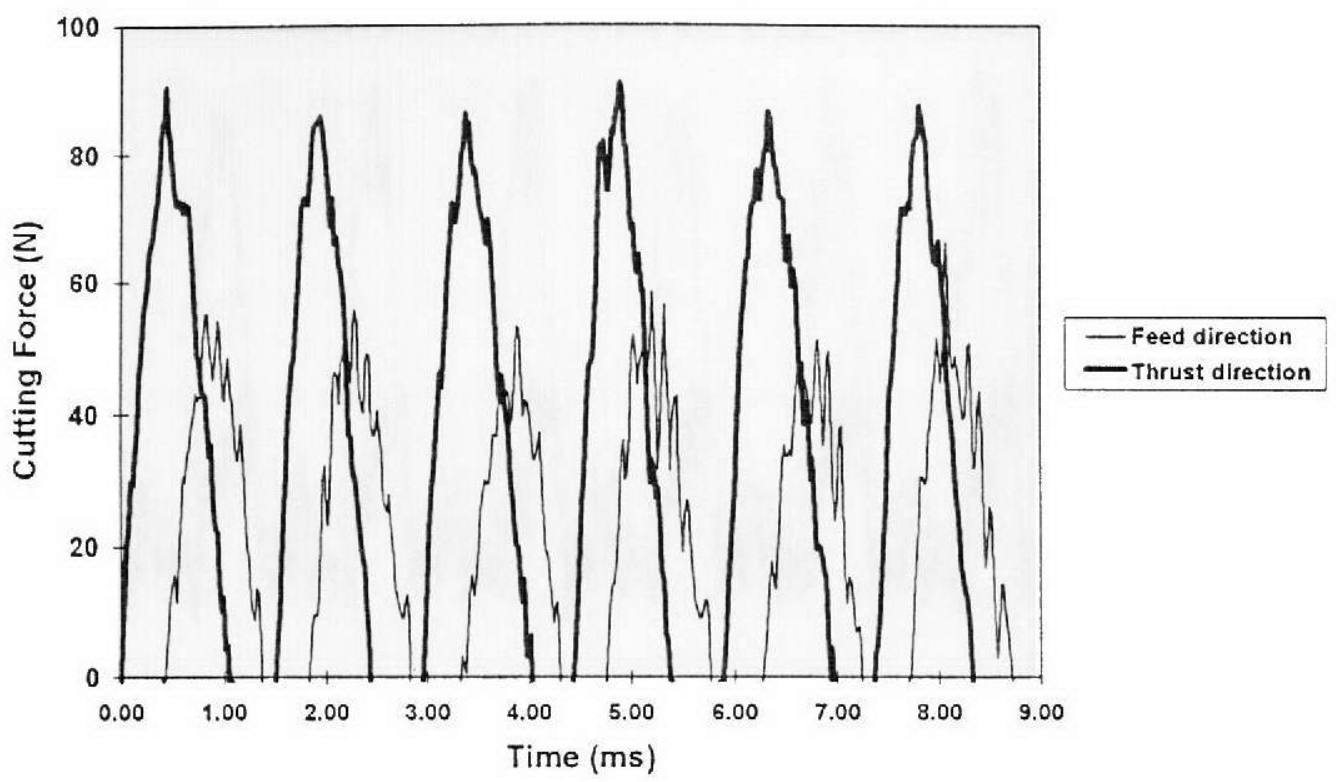

Figure 7.1 Experimental cutting forces of micro-end-milling without run-out

\section{Cutting Forces of Micro-End-Milling Operations}

2 flute $1 / 8^{\prime \prime}$ diameter carbide end mill, steel work-piece

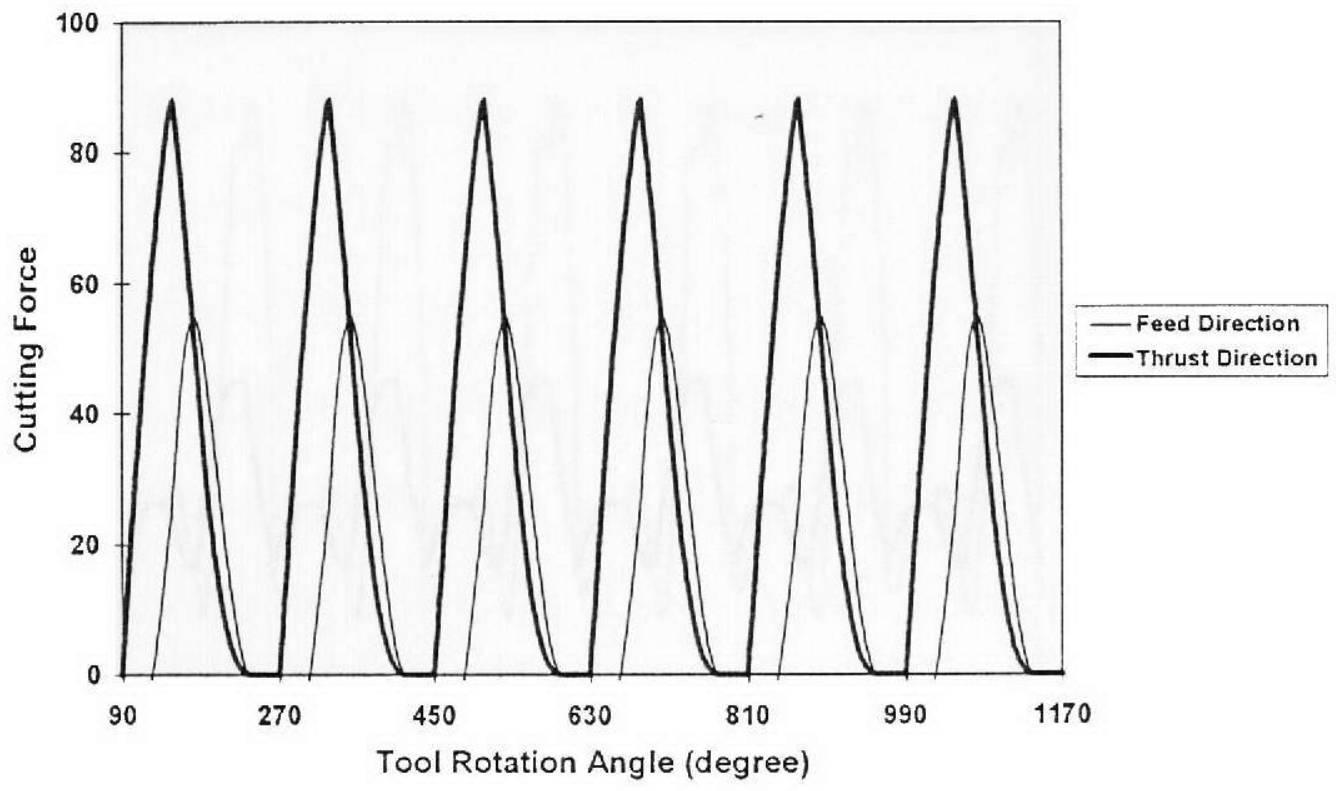

Figure 7.2 Analytical model based cutting forces of micro-end-milling without run-out 
Cutting Forces of Micro-End-Milling Operations

2 flute 1/16" diameter HSS end mill, POCO-3 work-piece

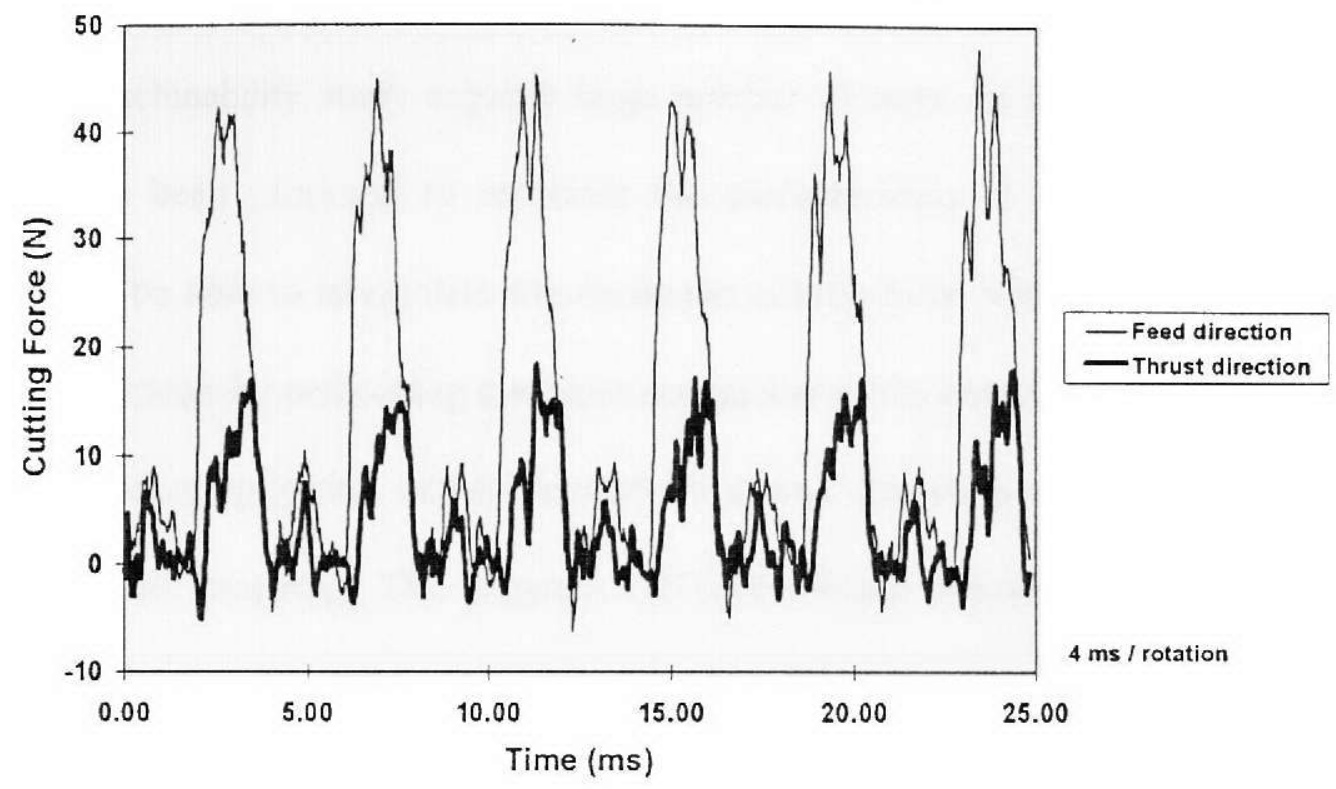

Figure 7.3 Experimental cutting forces of micro-end-milling with run-out

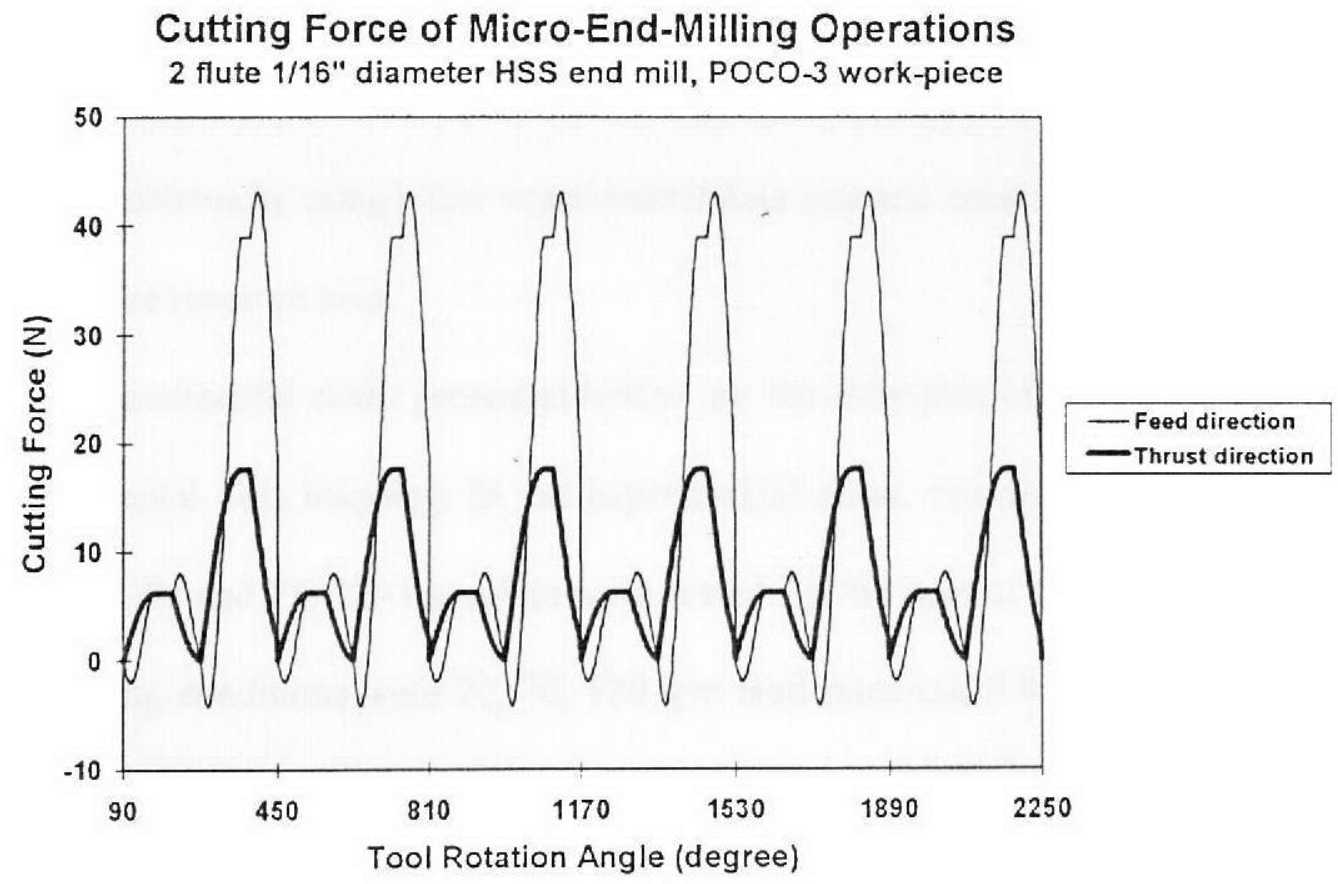

Figure 7.4 Analytical model based cutting forces of micro-end-milling with run-out 


\subsection{Representation of the Cutting Force Characteristics}

\section{Representation of the Cutting Force Characteristics by Using Neural Networks ${ }^{[16]}$}

The machinability study requires large number of tests. In this research, neural networks have been proposed to represent the characteristics of cutting forces. The objective is to be able to interpolate the maximum cutting force within the considerable tool parameter range by performing the minimum number of the experiments.

The back-propagation neural network is a well known powerful tool for the experimental data mapping. The program NNTool (Neural Network Research Tool) program was developed in 1995 and modified in 1996. The program was successfully used to determine the underground contamination distributions of New York area ${ }^{[14]}$ and Miami International Airport area ${ }^{[15[46]}$ In this study, the NNTool was used to estimate the maximum cutting force of micro-end-milling operations at different selected working conditions and tool diameters. ${ }^{[16]}$ The NNTool can automatically develop a good model of the research problems by using a few experimental data sets and create a maximum cutting force chart in the research area.

The experimental cases presented below are the examples of the neural network based experimental data mapping. In the experimental cases, two-flute $0.020 ", 0.0625$ " diameter end mills and POCO-3 graphite were tested. In the case of 0.020 " diameter end mill, the working conditions were $20,70,120 \mathrm{ipm}$ feed rates and 0.010 ", 0.030 ", 0.050 " depths of cut. In the case of 0.0625 " diameter end mill, the working conditions were 30 , 65,100 ipm feed rates and $0.0625 ", 0.100 ", 0.150$ " depths of cut. 15,000 rpm spindle speed and 50\% overlapped climbing end milling operations were used in the both cases. 
The cutting forces were found at 16 different working conditions. The experimental cutting forces of the selected working conditions are presented in Table 7.2 and 7.3.

\begin{tabular}{|c|c|c|c|}
\hline$X$ (thrust) direction cutting force $(\mathrm{N})$ & \multicolumn{3}{|c|}{ Feed rate (rpm) } \\
\hline Depth of cut (inch) & 20 & 70 & 120 \\
\hline 0.05 & 3.750 & 6.750 & non \\
\hline 0.03 & 4.850 & 6.500 & non \\
\hline 0.01 & 4.500 & 5.425 & 5.150 \\
\hline
\end{tabular}

\begin{tabular}{|c|c|c|c|}
\hline$Y$ (feed) direction cutting force (N) & \multicolumn{3}{|c|}{ Feed rate (rpm) } \\
\hline Depth of cut (inch) & 20 & 70 & 120 \\
\hline 0.05 & 8.250 & 16.500 & non \\
\hline 0.03 & 7.500 & 13.250 & non \\
\hline 0.01 & 5.000 & 8.750 & 10.000 \\
\hline
\end{tabular}

Table 7.2 Experimental data of 0.020 " diameter end mill machinability testing

\begin{tabular}{|c|c|c|c|}
\hline $\mathbf{X}$ (thrust) direction cutting force (N) & \multicolumn{3}{|c|}{ Feed rate (rpm) } \\
\hline Depth of cut (inch) & 30 & 65 & 100 \\
\hline 0.15 & 6.800 & 14.900 & 28.600 \\
\hline 0.1 & 6.800 & 12.700 & 19.900 \\
\hline 0.062 & 6.200 & 7.900 & 20.100 \\
\hline
\end{tabular}

\begin{tabular}{|c|c|c|c|}
\hline$Y$ (feed) direction cutting force & \multicolumn{3}{|c|}{ Feed rate (rpm) } \\
\hline Depth of cut (inch) & 30 & 65 & 100 \\
\hline 0.15 & 23.500 & 30.000 & 70.000 \\
\hline 0.1 & 16.250 & 24.500 & 42.500 \\
\hline 0.062 & 14.500 & 20.000 & 37.500 \\
\hline
\end{tabular}

Table 7.3 Experimental data of 0.0625 " diameter end mill machinability testing

The NNTool was used to develop an empirical model to evaluate the machinability of the whole selected test range by using the experimental data from very few test cases. The experimental data were collected at 7 and 9 different working conditions with 0.020 " and 0.0625 " end mills respectively. The feed rate and depth of cut were considered as two inputs of the neural network, and the tool cutting force was the output. A three-layer 
neural network with 10 hidden nodes was trained. The learning rate and momentum factor were 0.15 and 0.075 respectively. In the case of 0.020 " diameter tools, 13 data sets $(6$ experimental data and 7 boundary conditions) were used to train the neural network and 1 experimental data was reserved for its testing. In the case of 0.0625 " diameter tools, 9 experimental data sets were used to train the neural network. Two-dimensional neural network based empirical cutting force models of 0.020 " and 0.0625 " diameter end mills were developed with $8.4 \%$ and $4.8 \%$ average errors respectively when they were tested on the training cases. The model showed $15.7 \%$ error on the test case that was not used in the training. The results of 0.020 " diameter tools are presented in Figure 7.5 and 7.6 and the results of $0.0625 "$ diameter tool are presented in Figure 7.7 and 7.8.

Considering the tool diameter, another three-dimensional neural network was developed by using the data of all the 16 experiments. In the study, the three inputs to the neural network were the feed rate, depth of cut and tool diameter. The output was the tool cutting force. A ten-hidden-node three-layer neural network was designed. During the training of the neural network, a learning rate of 0.15 and a momentum factor of 0.075 were selected. Based on the experimental data, 30 data sets (16 experimental data and 14 boundary data) were used for the training of the neural network. The average error was less than $8.8 \%$. The results are presented in Figures 7.9 through 7.12 .

The results confirmed the accuracy of the neural network based experimental data mapping. The NNTool can be used to estimate the tool cutting forces with acceptable error in a selected range of parameters. 
Micro-tool Cutting Force in $X$ Direction

(15,000 rpm spindle speed, 0.020 inch HS steel tool, graphite workpiece)

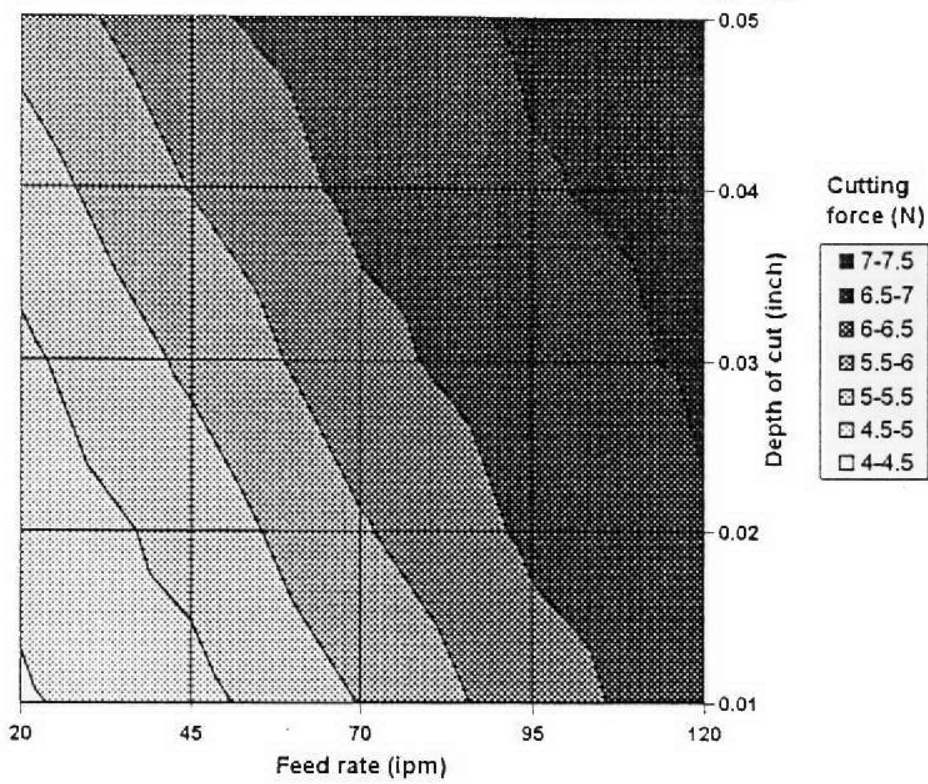

Figure 7.5 Thrust direction maximum cutting force of 0.020 " diameter end mills

Micro-tool Cutting Force In Feed Direction

(15,000 rpm spindle speed, 0.020 inch $\mathrm{HS}$ steel tool, graphite workpice)

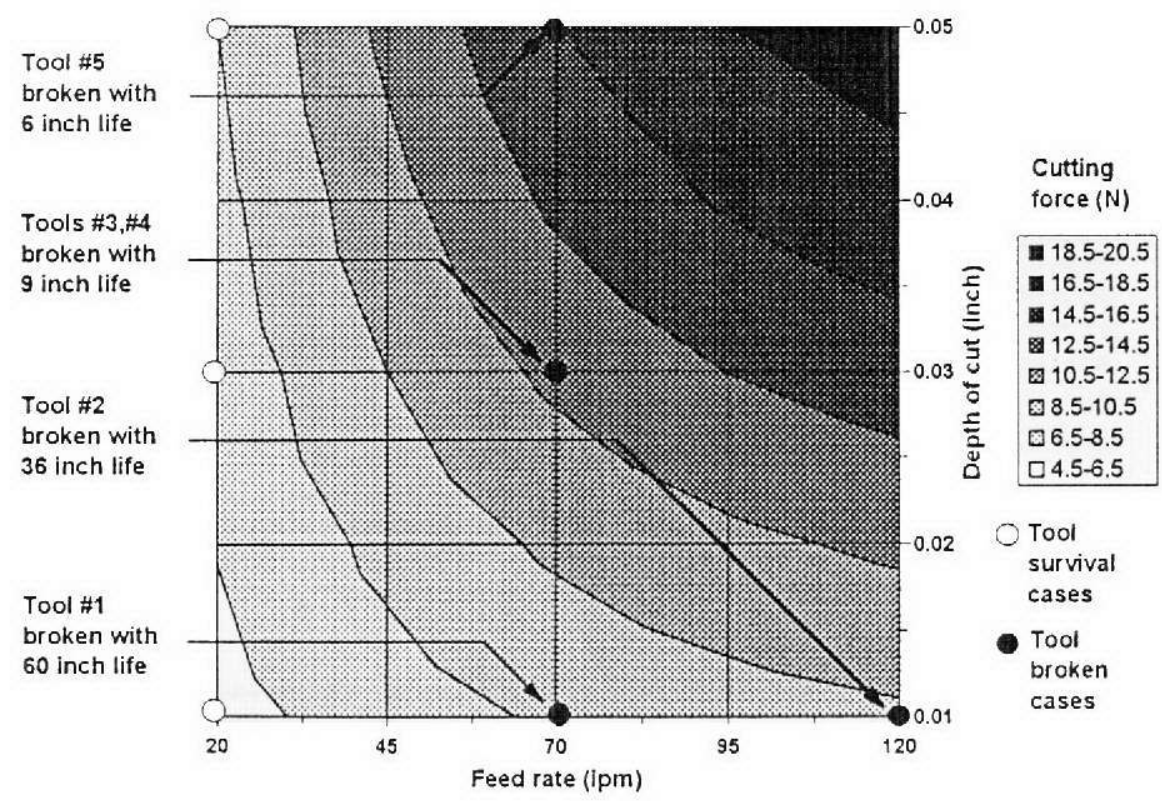

Figure 7.6 Feed direction maximum cutting force of 0.020 " diameter end mills 
Micro-tool Cutting Force in $\mathrm{X}$ Direction

(15,000 rpm spindle speed, 0.0625 inch HS steel tool, graphite workpiece)

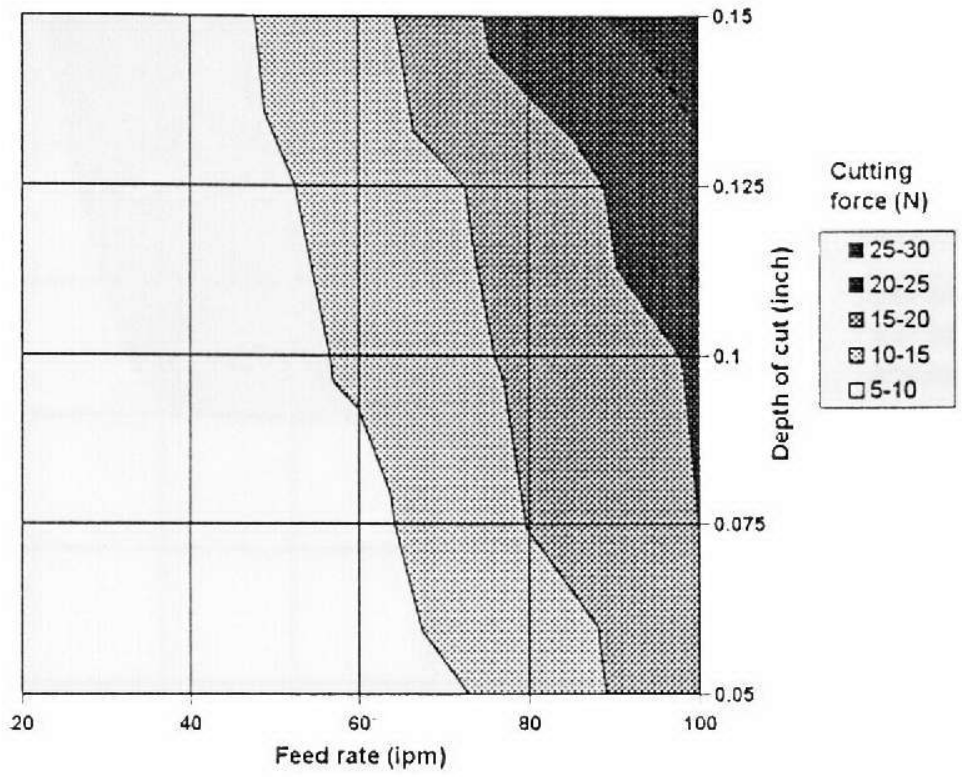

Figure 7.7 Thrust direction maximum cutting force of 0.0625 " diameter end mills

Micro-tool Cutting Force In Feed Direction

(15,000 rpm spindle speed, 0.0625 inch dia. tool, graphite)

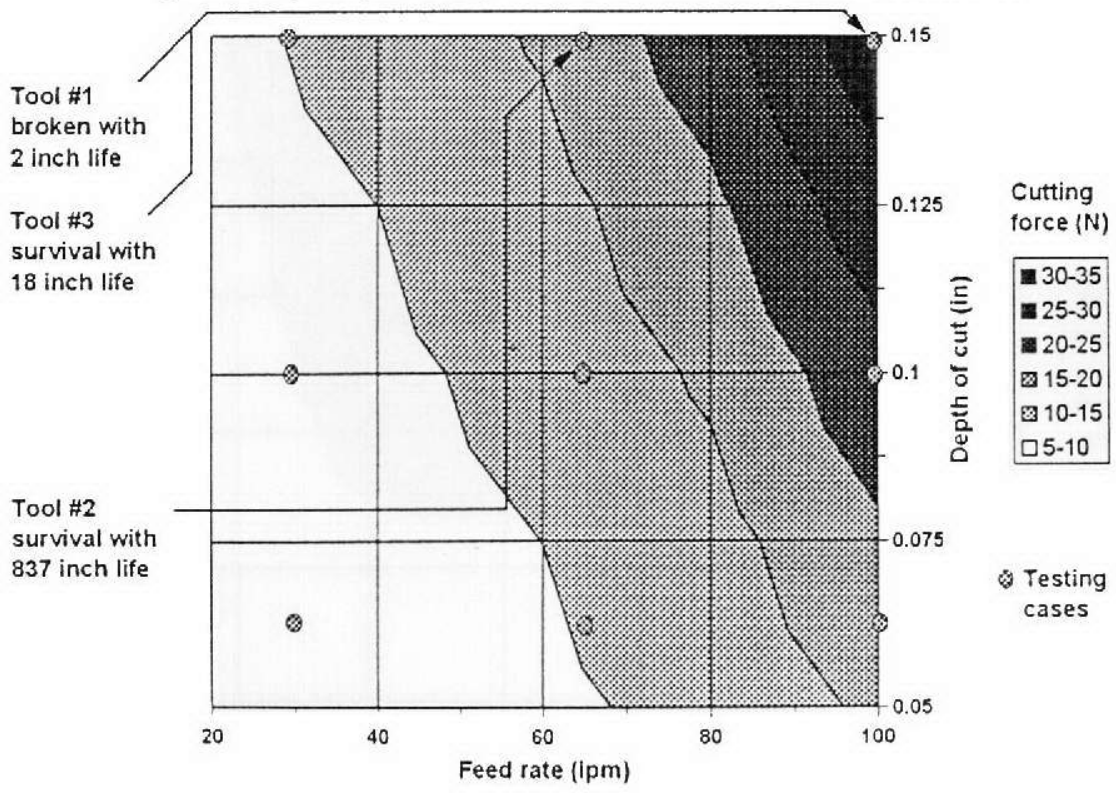

Figure 7.8 Feed direction maximum cutting force of 0.0625 " diameter end mills 
Micro-tool Cutting Force In Feed Direction

POCO 3 workpiece, 0.020 inch diameter high speed steel end mill

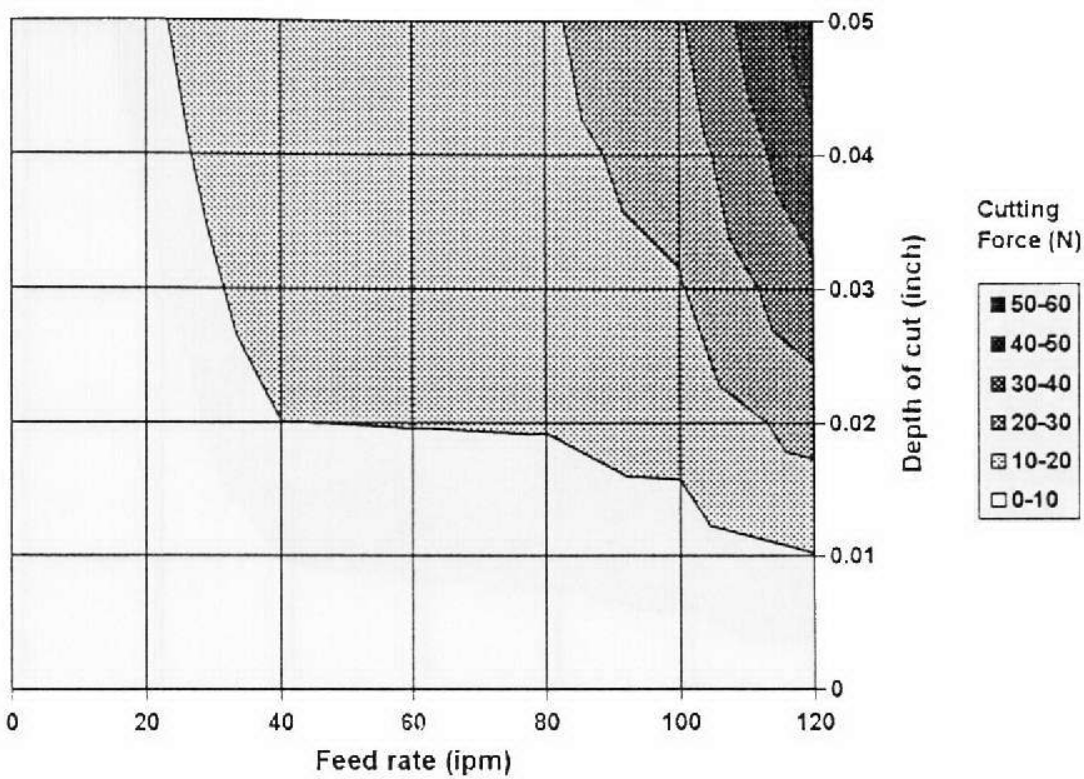

Figure 7.9 Feed direction maximum cutting force of 0.020 " diameter end mill

Micro-tool Cutting Force In Feed Direction POCO 3 workpiece, 0.030 inch diameter high speed steel end mill

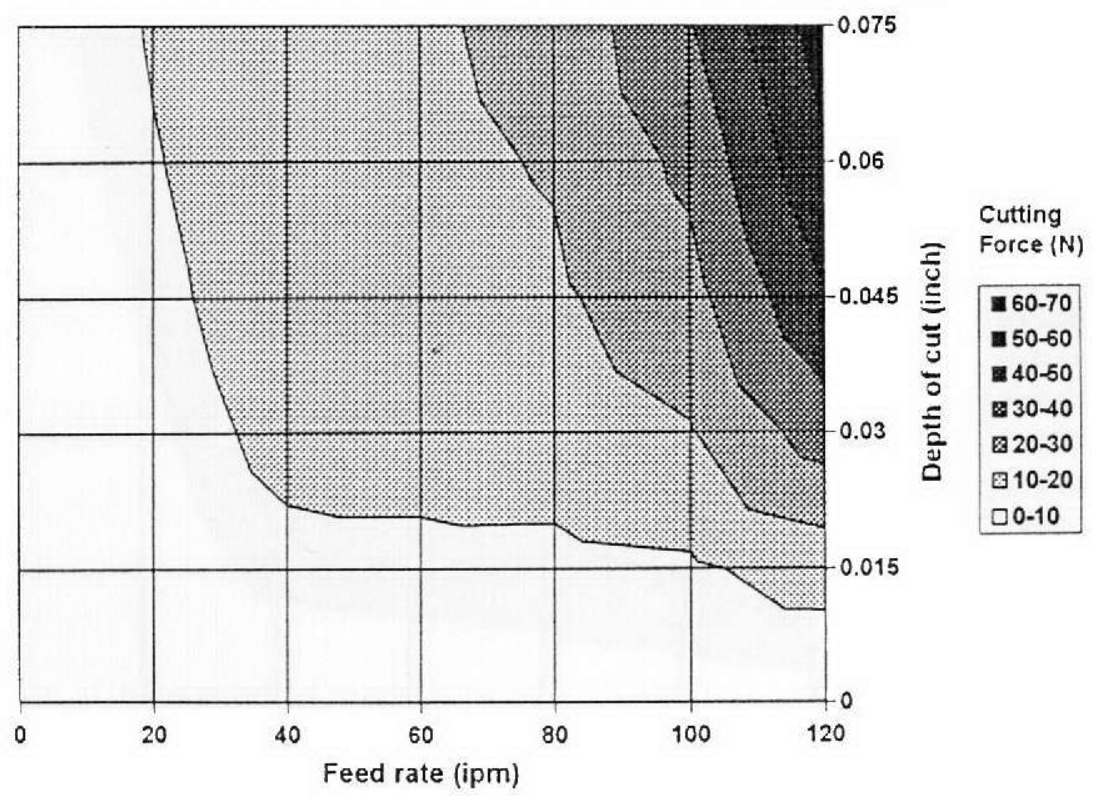

Figure 7.10 Feed direction maximum cutting force of 0.030 " diameter end mill 
Micro-tool Cutting Force In Feed Direction

POCO 3 workpiece, 0.050 inch diameter high speed steel end mill

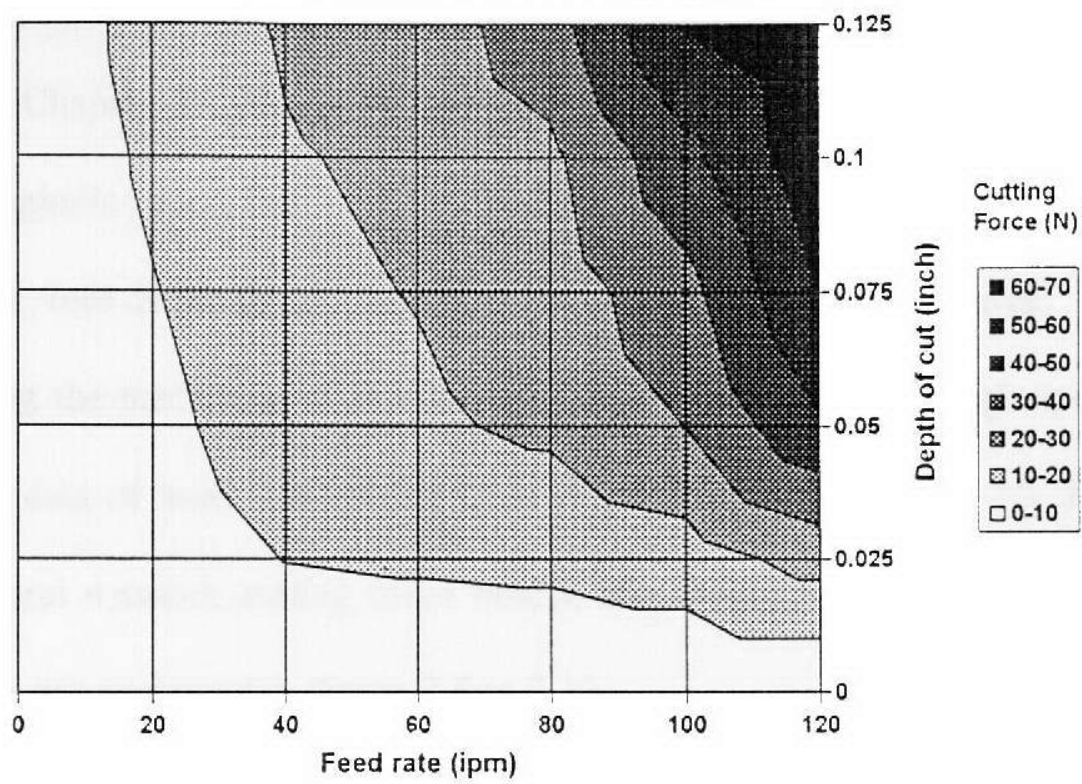

Figure 7.11 Feed direction maximum cutting force of 0.050 " diameter end mill

Micro-tool Cutting Force In Feed Direction

POCO 3 workpiece, 0.0625 inch diameter high speed steel end mill

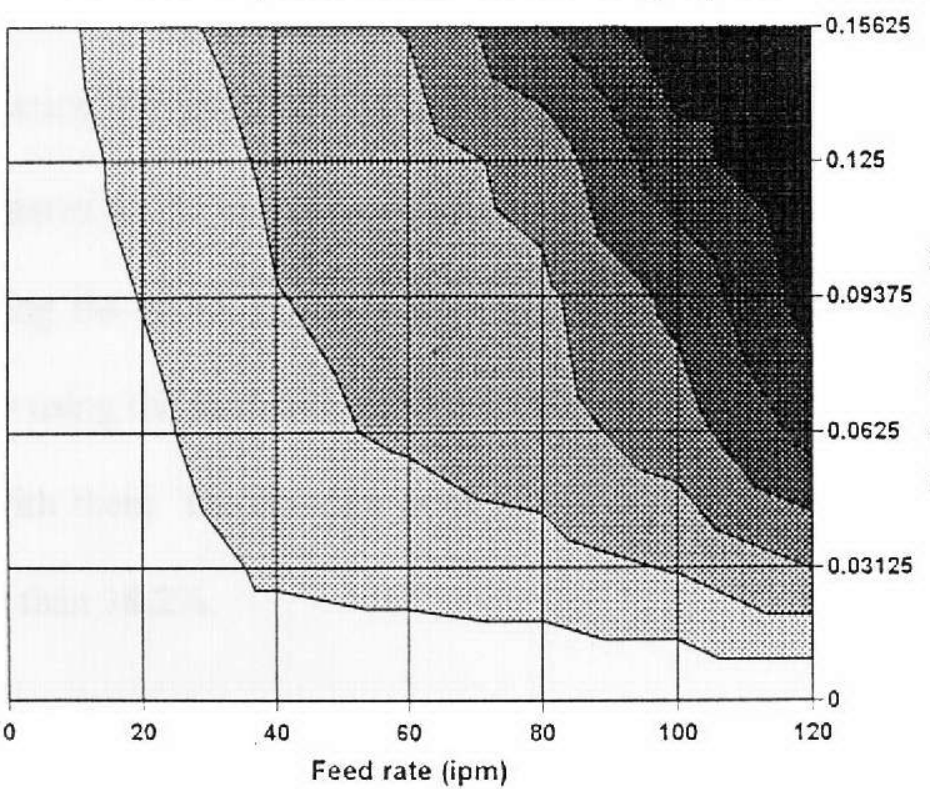

Cutting

Force (N)

\begin{tabular}{|c|}
\hline$=60-70$ \\
\hline च $50-60$ \\
\hline $\mathbf{1} 40-50$ \\
\hline $30-40$ \\
\hline $20-30$ \\
\hline (지 10-20 \\
\hline$\square 0-10$ \\
\hline
\end{tabular}

Figure 7.12 Feed direction maximum cutting force of 0.0625 " diameter end mill 


\section{Representation of the Cutting Force Characteristics by Using Analytical Model}

The calculation of the cutting forces by using the analytical model has been presented in Chapter IV (Figure 4.7 to 4.27 ). The parameters discussed in the model includes the spindle speed, feed rate, depth of cut, run-out and it's angle, tool cutting entry and exit angle, tool diameter, tool helix angle and the numbers of tool flutes.

During the machining of POCO-3 graphite work-piece with high speed tool, the experimental data of maximum cutting force is listed in Table 7.2 and 7.3. Based on the empirical neural network cutting force model, the characteristics of the tool maximum cutting forces are presented in Figure 7.5 to 7.12.

Considering the case of 0.020 " diameter end mill and POCO-3 graphite workpiece, the characteristics of the tool maximum cutting forces can be calculated from the analytical model. The working conditions are $15,000 \mathrm{rpm}$ spindle speed, 20 to $120 \mathrm{ipm}$ feed rate, $0.01 "$ to $0.05 "$ depth of cut and $50 \%$ overlapped climbing milling. The thrust and feed direction maximum cutting forces are presented in Figures 7.13 and 7.14.

Compared to the experimental cutting forces (see Table 7.2) and estimated cutting forces by using the neural network based model (Figure 7.5 and 7.6 ), the cutting forces calculated by using the analytical cutting force model (Figure 7.13 and 7.14) have a good agreement with them. The average error of the maximum cutting forces of the analytical model is less than $38.2 \%$. 


\section{Thrust Direction Maximum Cutting Force of Micro-End-milling Operations}

(2 flute 20" diameter HSS end mill, POCO-3 graphite work-piece)

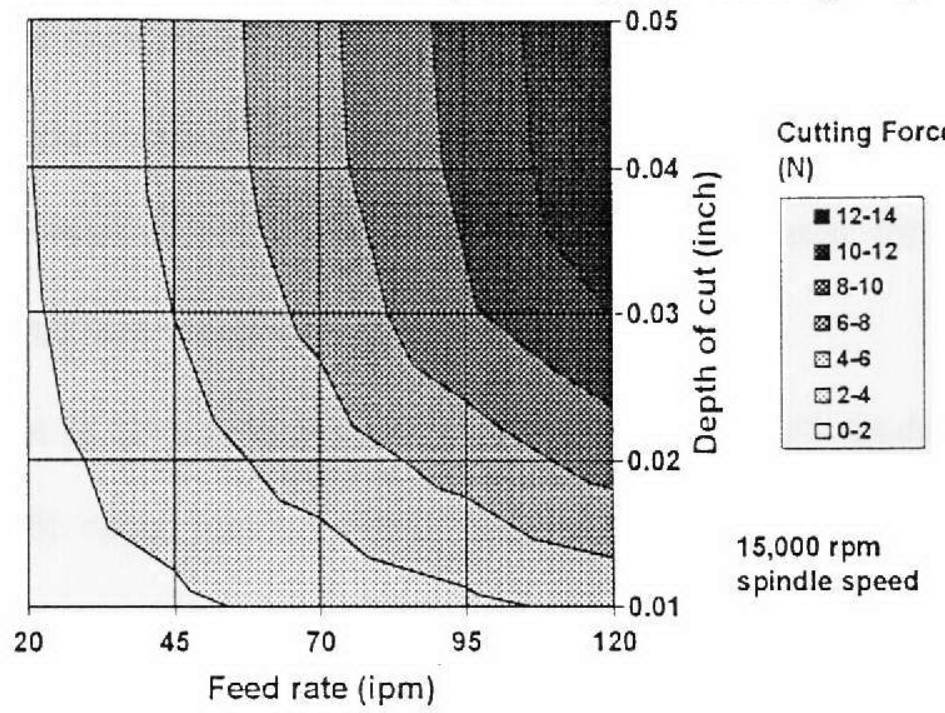

Figure 7.13 Model based thrust direction maximum cutting force of micro-end-milling

\section{Feed Direction Maximum Cutting Force of Micro-End-milling Operations}

(2 flute 20" diameter HSS end mill, POCO-3 graphite work-piece)

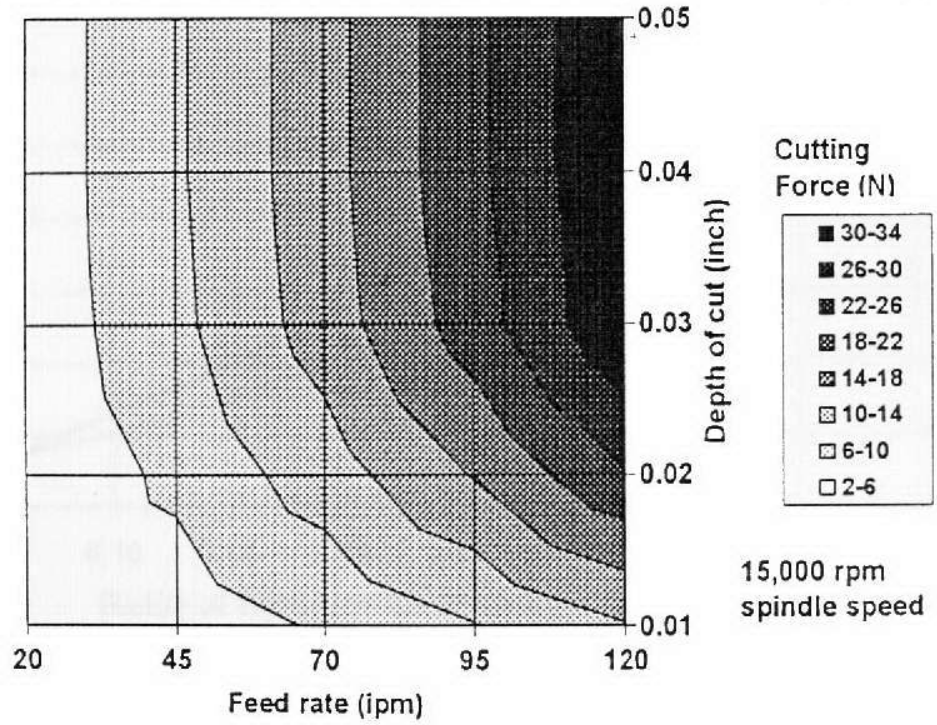

Figure 7.14 Model based feed direction maximum cutting force of micro-end-milling 


\section{Difference Between Micro and Conventional End Milling Operations}

From formulas 7.1 .1 to 7.1 .8 , it can be easily understood that micro-end-milling operations has the same cutting force values as conventional end milling operations if the ratio of the feed per tooth to the tool's radius $\left(f_{t} / r\right)$ is equal to zero. It is suggested that database of conventional end milling operations could be used for micro-end-milling operations when $f_{t} / r$ were small enough to be neglected.

Two cases of the simulated cutting force ratio of the both models are presented in Figure 7.15. In the cases, tool had 0.020 " diameter. The cutting conditions were 15,000 rpm spindle speed, 0 to 150 ipm feed rate and 0.010 " and 0.020 " depth of cut.

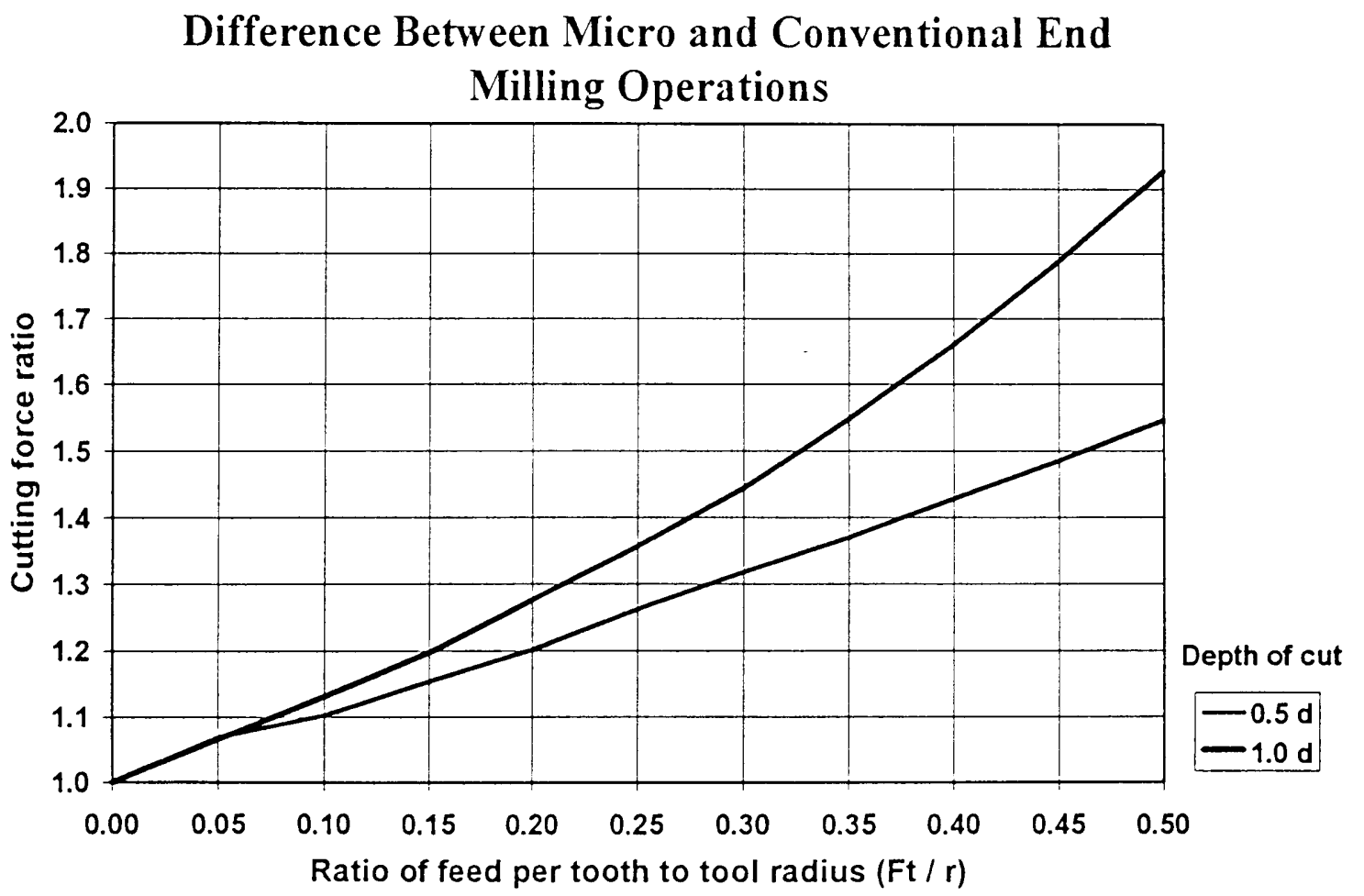

Figure 7.15 Difference between micro and conventional end milling operations 
The cutting force calculated by using conventional end milling model $\left(\mathfrak{f}_{\mathbf{t}} / \mathrm{r}=0\right)$ is always smaller than the one calculated by micro-end-milling model (considering $f_{t} / r$ ). If $f_{t} / r$ $<0.1$, the difference of maximum cutting force between the both models is less than $10 \%$. In other word, the both models have very similar performance when $\mathrm{f}_{\mathrm{t}} / \mathrm{r}<0.1$. The database of conventional end milling operations could be used for micro-end-milling operations with a $10 \%$ difference of the maximum cutting force. If the conclusion could proved experimentally, it would save a lot of experimental time and money for the investigation of micro-end-mill machinability. 


\subsection{Performance of Monitoring in Micro-End-Milling Operations}

\section{Tool Breakage Detection ${ }^{[27-30]}$}

Tool breakage detection methods that use acoustic emission (AE) signal have been discussed in Chapter $\mathrm{V}$. The procedures of the $\mathrm{AE}$ based tool breakage detection are simple and efficient. Two statistics algorithms of the tool breakage detection have been developed for two different working conditions. They accurately detected the tool breakage in all the studied cases with a very fast response capability. Two experimental cases were presented in Chapter V. Two-flute 0.015 " diameter high speed steel end mill cut on mild steel work-piece were tested. The working conditions were $30,000 \mathrm{rpm}$ spindle speed, $0.24 \mathrm{ipm}$ feed rate and 0.016 " depth of cut in the experiment I, 3,000 rpm spindle speed, $0.9 \mathrm{ipm}$ feed rate and 0.005 " depth of cut in the experiment II.

The data pattern of the experiment I was simple and tool breakage was detected by the detecting method I (see Chapter V). The data pattern of the experiment II was more complicated and difficult to distinguish the difference between tool broken and leaving the work-piece. The method II successfully detected the tool breakage in all of the cases of the experiment II. The test results are presented in Figure 7.16 (tool broken case) and Figure 7.17 (tool left from work-piece case), where the test procedures are shown under the $\mathrm{AE}$ signals. The down points of the line were testing points. The line was down to the level 1 when the tool left the work-piece and the level 2 when it was broken.

The results of monitoring tool breakage by the tool cutting force have been discussed in Chapter V. It has been proved that tool cutting force can be used not only to detect but also predict the tool breakage. 


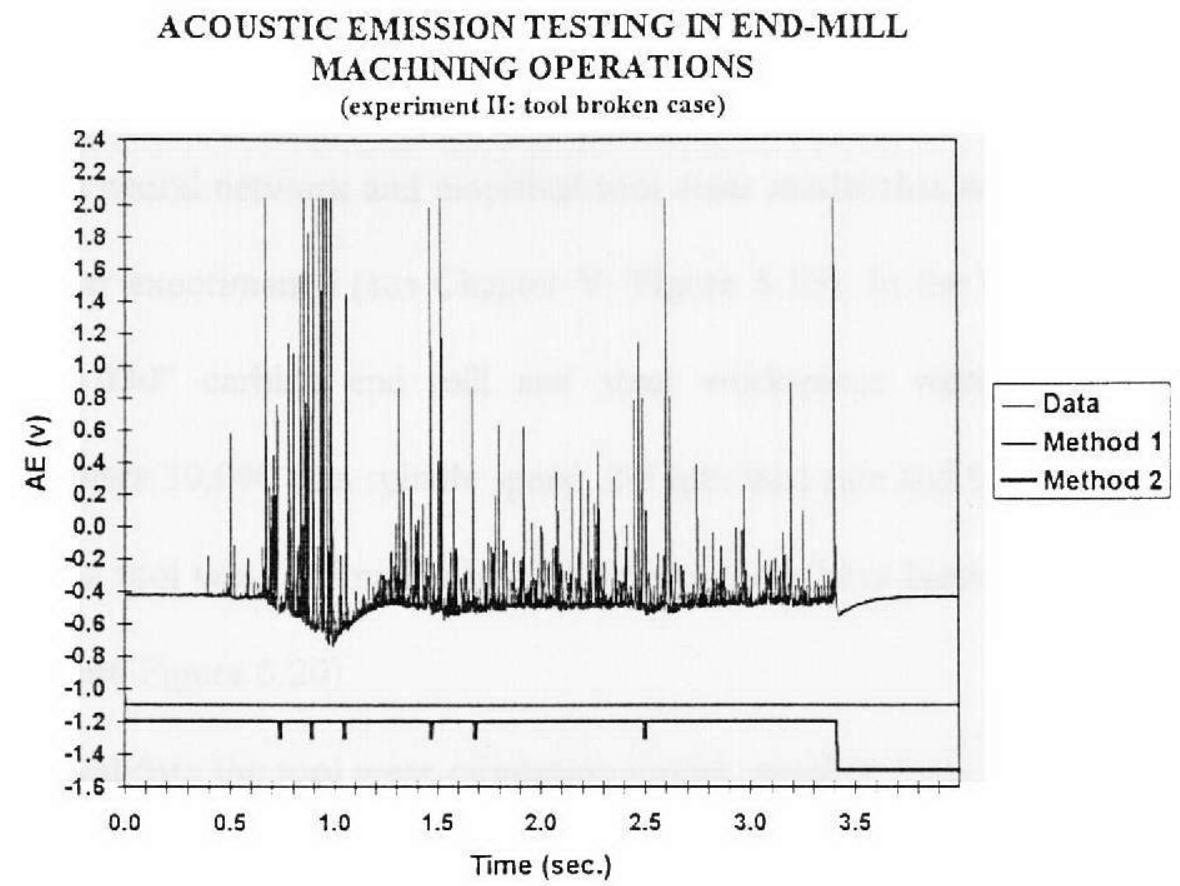

Figure 7.16 Tool breakage monitoring by AE activity of experiment II, case 1

\section{ACOUSTIC EMISSION TESTING IN END-MILL MACHINING OPERATIONS}

(experiment II: tool left from the work-piece case)

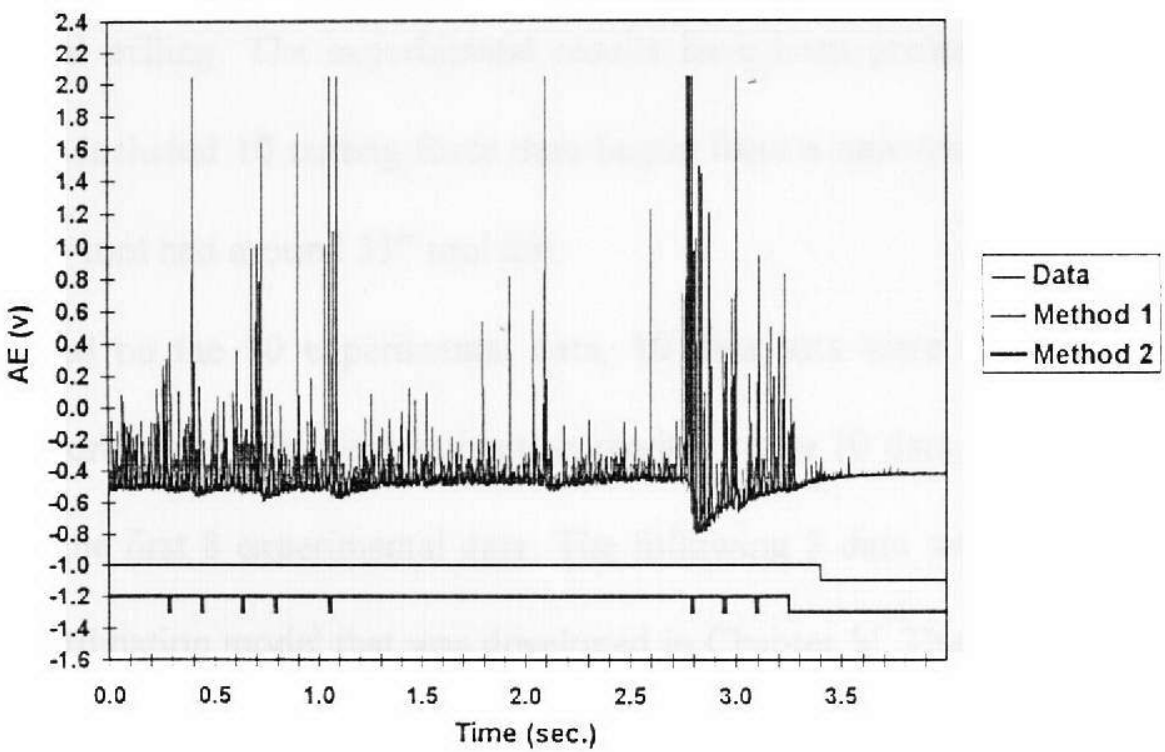

Figure 7.17 Tool breakage monitoring by AE activity of experiment II, case 2 
2. Tool Wear Monitoring and Estimation ${ }^{[39][42]}$

A tool wear estimation model has been developed in Chapter $\mathrm{V}$ by using the backpropagation neural network and empirical tool wear model that was based on the data of the tool wear experiment I (see Chapter V, Figure 5.15). In the tool wear experiment I, two-flute 0.030 " carbide end mill and steel work-piece were tested. The working conditions were $30,000 \mathrm{rpm}$ spindle speed, $2.5 \mathrm{ipm}$ feed rate and 0.045 " depth of cut. The results of the tool wear estimation by tool cutting force have been presented in Chapter $\mathrm{V}$ (Table 5.1 and Figure 5.20).

To validate the tool wear estimation model, another case, tool wear experiment II (see Chapter V, Figure 5.15), was tested. The data of the experiment II never be used when the tool wear estimation model was generated. In the experiment II, two-flute 0.030 " carbide end mill and steel work-piece were tested. The working conditions were $20,000 \mathrm{rpm}$ spindle speed, $1.25 \mathrm{ipm}$ feed rate, 0.0225 " depth of cut and $50 \%$ overlapped climbing end milling. The experimental results have been presented in Figure 5.15 and 5.16, which included 10 cutting force data begun from a new tool and ended until it was broken. The tool had around 33" tool life.

Based on the 10 experimental data, 10 data sets were used for the test of the neural network based tool wear estimation model. In the 10 data sets, the first 7 data sets were from the first 8 experimental data. The following 3 data sets were estimated by the tool wear estimation model that was developed in Chapter V. The last 2 experimental data were reserved for the test purpose. In other word, in the study the tool life was estimated after the tool had cut a 27 "-long work-piece. 


\begin{tabular}{|c|c|c|c|c|c|}
\hline & \multicolumn{5}{|c|}{ Cutting Force (N) } \\
\hline $\begin{array}{c}\text { Tool Life } \\
\text { (inch) }\end{array}$ & $\begin{array}{c}\text { Experimental } \\
\text { Data }\end{array}$ & $\begin{array}{c}\text { Empirical } \\
\text { Model }\end{array}$ & $\begin{array}{c}\text { Estimation } \\
\text { Data }\end{array}$ & $\begin{array}{c}\text { Empirical } \\
\text { Model Error }\end{array}$ & $\begin{array}{c}\text { Estimation } \\
\text { Error }\end{array}$ \\
\hline 3 & & & & & \\
\hline 6 & 28.080 & 27.807 & & 0.273 & \\
\hline 9 & 25.360 & 27.839 & & -2.479 & \\
\hline 12 & 25.920 & 27.935 & 25.234 & -2.015 & 0.687 \\
\hline 15 & 29.320 & 28.150 & 26.577 & 1.170 & 2.743 \\
\hline 18 & 29.560 & 28.566 & 28.911 & 0.994 & 0.649 \\
\hline 21 & 28.840 & 29.283 & 28.699 & -0.443 & 0.141 \\
\hline 24 & 31.240 & 30.428 & 29.491 & 0.812 & 1.749 \\
\hline 27 & 31.320 & 32.154 & 31.703 & -0.834 & -0.383 \\
\hline 30 & 33.160 & 34.640 & 33.051 & -1.480 & 0.109 \\
\hline 33 & 38.200 & 38.093 & 34.871 & 0.107 & 3.329 \\
\hline 36 & & & 36.195 & & \\
\hline 39 & & & 39.012 & & \\
\hline Average & 27.976 & & & 1.980 & 2.255 \\
\hline Persentage & & & & $7.08 \%$ & $8.06 \%$ \\
\hline
\end{tabular}

Table 7.4 Results of the tool wear forecasting model testing

Tool Wear Estimation Testing

2 flute 0.030 " diameter carbide end mill, steel work-piece

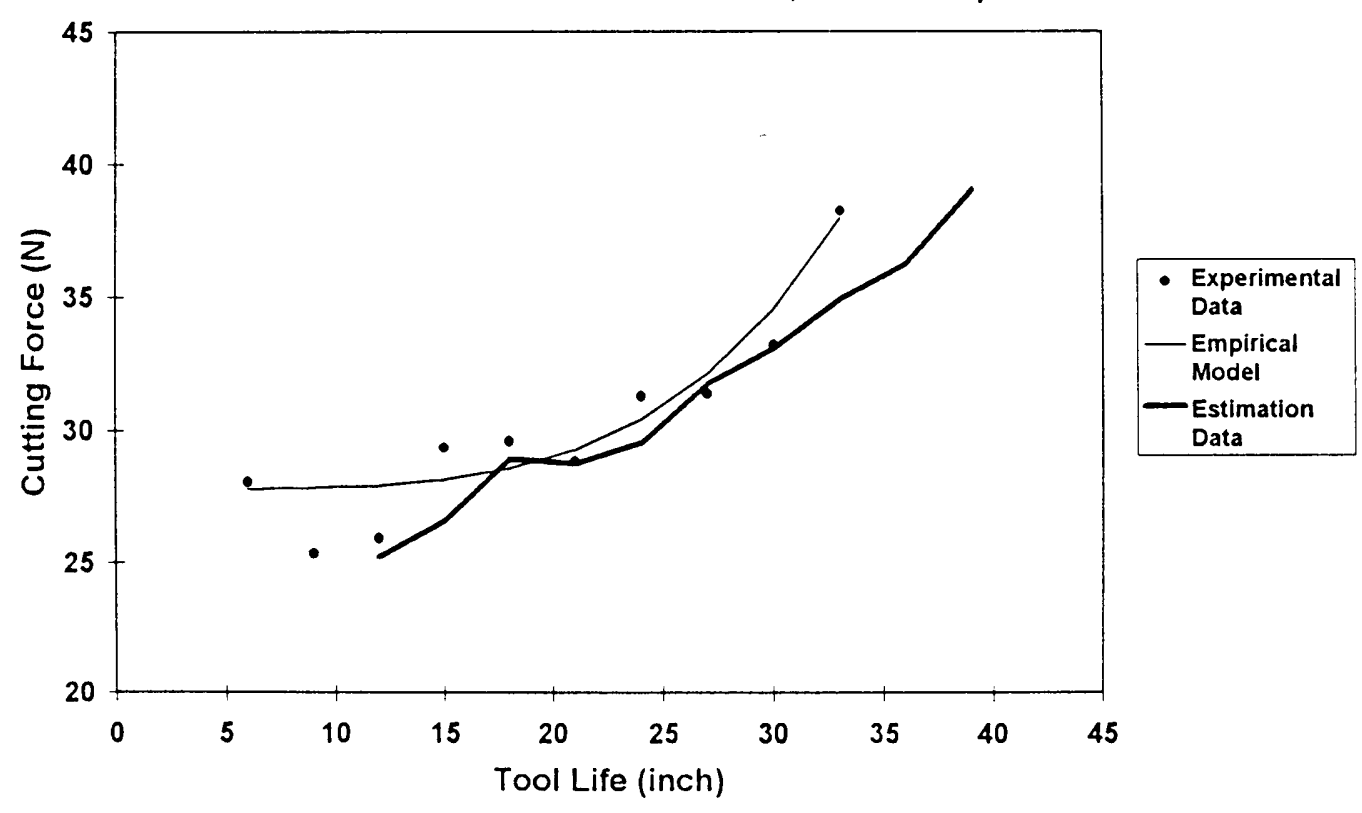

Figure 7.18 Performance of the tool wear forecast model 
The test results of the tool wear estimation model are presented in Table 7.4 and Figure 7.18. The cutting force was estimated with $1.8 \mathrm{~N}$ or $6.45 \%$ average error. The empirical tool wear model shown in Figure 7.18 was developed from the 10 experimental data after the experiment had been completed. It was very similar to the model developed in Chapter V. Only the basic cutting force level $\mathrm{C}_{1}$ was different because of the different working conditions. The coefficients of empirical tool wear model were:

$$
\begin{aligned}
& C_{1}=27.800 \\
& C_{2}=0.0522 \\
& C_{3}=4.287
\end{aligned}
$$

If the tool breakage critical cutting force was selected as $38 \mathrm{~N}$, the tool life would be estimated as around 37.5 inch. Compared to the actual tool life, 33 inch, the tool life estimated by the tool wear estimation model had an acceptable error, $13.6 \%$.

The tool breakage critical cutting force can be determined by the experimental data. To improve the accuracy of tool wear estimation, the tool wear empirical and estimation model should be improved further based on experimental observations.

Considering the tool wear coefficient $\mathrm{K}_{\mathrm{w}}$ in formula 5.2.3, the analytical cutting force model has been modified to include the tool wear. The cutting forces of two cases, the new tool case and pre-failure case of the tool wear experiment I (see Chapter V, Figure 5.15), were presented in Figure 7.19 and 7.20, in which they were compared with the cutting forces of the analytical model. 


\section{Cutting Force of Micro-End-Milling Operations}

in Tool Wear Experiments

(new tool)

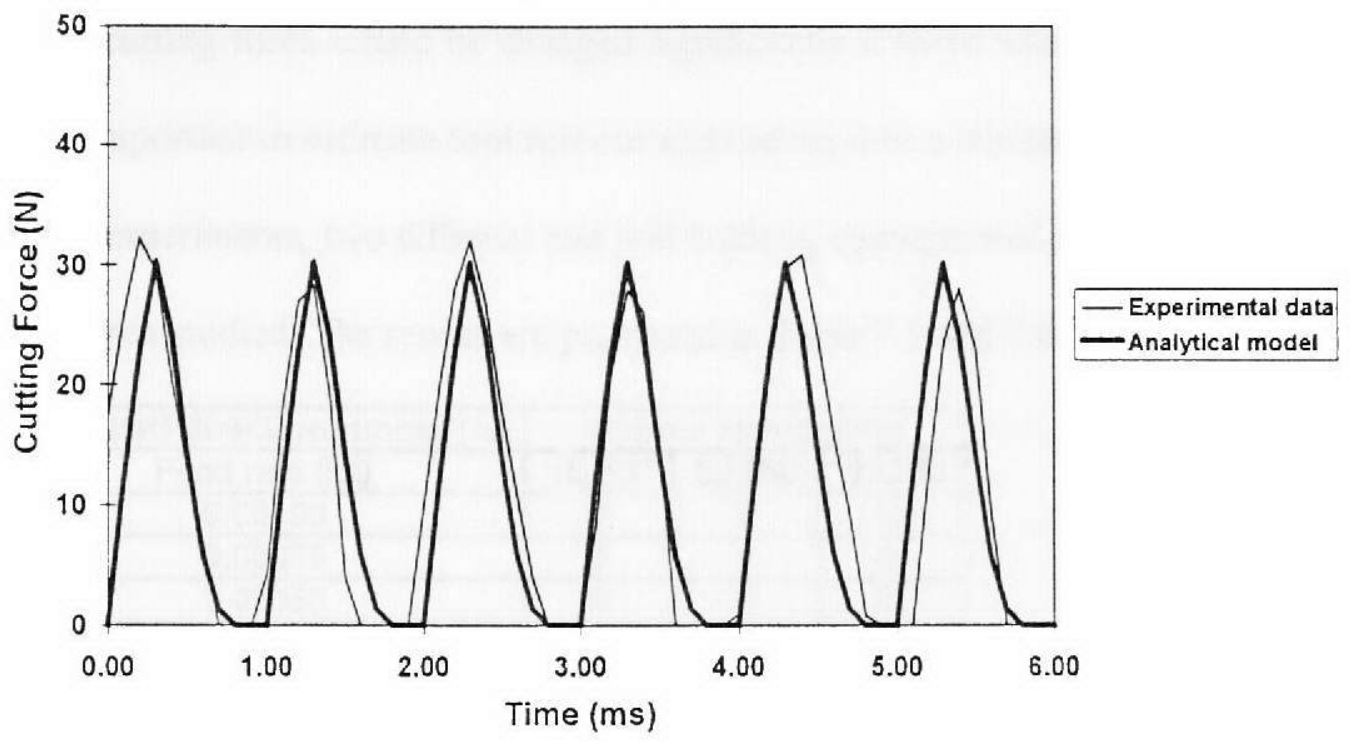

Figure 7.19 Thrust direction cutting force of the new tool

Cutting Force of Micro-End-Milling Operations in Tool Wear Experiments

(tool before breakage)

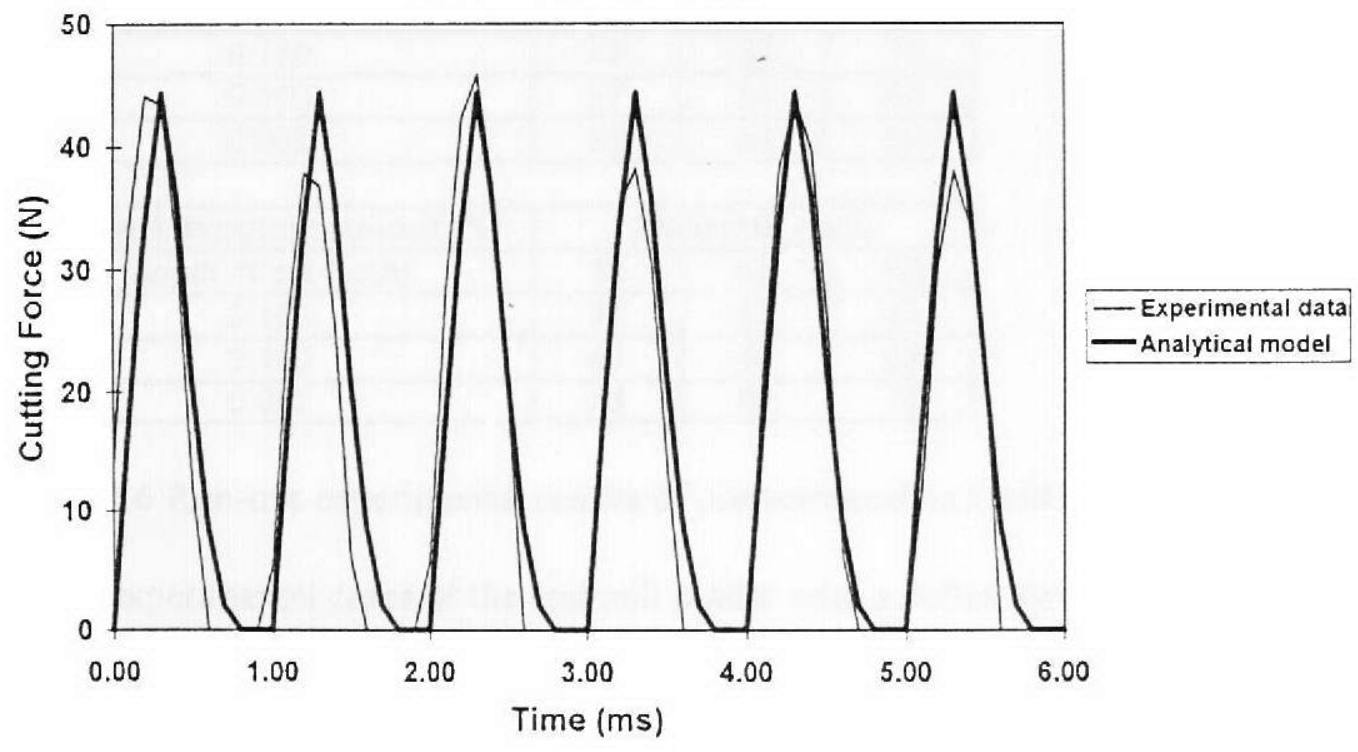

Figure 7.20 Thrust direction cutting force of the tool before breakage 


\section{Tool Run-out Estimation and Cutting Condition Monitoring ${ }^{[19]}$}

Tool run-out exists in most of micro-end-milling operations. Because of tiny tool diameter, the cutting force would be changed significantly if there was only a small tool run-out. It is important to estimate tool run-out and reduce it to a minimum level.

In the experiments, two different end mill holders, conventional and collet end mill holder, have been studied. The results are presented in Table 7.5 and 7.6.

\begin{tabular}{|c|c|c|c|}
\hline$X$ (thrust) direction runout (\%) & \multicolumn{3}{|c|}{ Spindle speed (rpm) } \\
\hline Feed rate (ipt) & 15,000 & 32,000 & 50,000 \\
\hline 0.00100 & 9 & 9 & 34 \\
\hline 0.00075 & 8 & 9 & 49 \\
\hline 0.00050 & 0 & 0 & 35 \\
\hline
\end{tabular}

\begin{tabular}{|c|c|c|c|}
\hline$Y$ (feed) direction runout (\%) & \multicolumn{3}{|c|}{ Spindle speed (rpm) } \\
\hline Feed rate (ipt) & 15,000 & 32,000 & 50,000 \\
\hline 0.00100 & 48 & 10 & 20 \\
\hline 0.00075 & 50 & 65 & 47 \\
\hline 0.00050 & 63 & 55 & 23 \\
\hline
\end{tabular}

Table 7.5 Run-out experimental results of collet end mill holder

\begin{tabular}{|c|c|c|c|}
\hline X (thrust) direction runout (\%) & \multicolumn{3}{|c|}{ Feed rate (ipm) } \\
\hline Depth of cut (inch) & 30 & 65 & 100 \\
\hline 0.150 & 72 & 85 & 71 \\
\hline 0.100 & 57 & 54 & 64 \\
\hline 0.062 & 56 & 83 & 60 \\
\hline
\end{tabular}

\begin{tabular}{|c|c|c|c|}
\hline$Y$ (feed) direction runout (\%) & \multicolumn{3}{|c|}{ Feed rate (ipm) } \\
\hline Depth of cut (inch) & 30 & 65 & 100 \\
\hline 0.150 & 87 & 87 & 46 \\
\hline 0.100 & 40 & 48 & 63 \\
\hline 0.062 & 78 & 86 & 79 \\
\hline
\end{tabular}

Table 7.6 Run-out experimental results of conventional end mill holder

In the experimental cases of the end mill holder with a collet, two-flute carbide end mill with 0.0625 " diameter is used to cut aluminum work-piece. The working conditions were following: $15,000,32,000$ and $50,000 \mathrm{rpm}$ spindle speed, $0.0005,0.00075$ and 0.001 ipm feed rate, 0.020 " depth of cut and $50 \%$ overlapped climbing milling. In the cases of 
the conventional end mill holder (holding the tool with set-screw), POCO-3 graphite work-piece was cut by a high speed end mill with two-flute 0.0625 " tool diameter. The working conditions were $15,000 \mathrm{rpm}$ spindle speed, 30,65 and $100 \mathrm{ipm}$ feed rate, $0.0625 ", 0.100 "$ and 0.150 " depth of cut, and 50\% overlapped climbing milling.

Based on the experimental results, it is known that run-out of micro-tools effect cutting performance more serious than conventional tools. The tested end mill holder with a collet had $0 \%$ to $65 \%$ run-out and the average was around $30 \%$ average. The tested end mill holder with set-screw had $40 \%$ to $87 \%$ run-out and the average was around $68 \%$. The collet end mill holder is much better than the conventional one.

Based on the analytical cutting force model, estimated the tool run-out was studied in Chapter IV. When the tool run-out angle is parallel to the tool cutting edges $(\gamma=0)$, the cutting force has a maximum value that almost double of the minimum value (see Chapter IV, Figure 4.18) and the work-piece surface has the lowest precision. When the tool runout angle is perpendicular to the tool cutting edges $(\gamma=\pi / 2)$, the cutting force has a minimum value and the work-piece surface has the highest precision.

If end mill holder with a set screw is going to be used, the tool should be screwed on the holder perpendicular to the cutting edges of the two-flute tool. The same angle should be 45 degree in the case of the four-flute tool. These adjustments would reduce the run-out to the minimum level.

It is important to estimate the tool run-out and monitor the tool cutting conditions of micro-end-milling operations. The method of estimating tool run-out and monitoring tool cutting conditions by using the genetic algorithm with the analytical cutting force 
model has been discussed in Chapter V. The main idea is to compare the experimental cutting forces to the cutting forces calculated by the analytical model, and use the genetic algorithm to search the optimal fitting variables of the parameters (tool run-out or cutting conditions) of the analytical model. The method can be used to estimate the tool run-out, spindle speed, feed rate, depth of cut, entry and exit cutting angles. The details were discussed in Chapter V.

The genetic evolution procedure of tool run-out estimation had been presented in Figure 5.23 of Chapter V. The results are presented in Table 7.7. The error is less than $2.5 \%$ after 210 generations.

\begin{tabular}{|l|c|c|c|}
\hline & Acutal Value & Estimating Value & Error \\
\hline Tool Run-out (inch) & 0.001 & 0.00099 & $1.00 \%$ \\
\hline Tool Run-out Angle (degree) & 30 & 29.26 & $2.47 \%$ \\
\hline Optimal Function Fitness & 0 & 0.0031 & 0.0031 \\
\hline
\end{tabular}

Table 7.7 Results of the tool run-out estimation

The results of the tool cutting condition monitoring have been presented in Table 5.2 of Chapter V. The error is reduced to less than $3 \%$ in 20 generations and less than $1 \%$ in 120 generations. The average errors of all the studied cases are $1.13 \%$ at the $20^{\text {th }}$ generation and $0.19 \%$ at $120^{\text {th }}$ generation, which are presented in Table 7.8.

\begin{tabular}{|l|c|c|}
\hline & Error in 20th Generation & Error in 120th Generation \\
\hline Tool Cutting Angle & $1.13 \%$ & $0.19 \%$ \\
\hline Optimal Function Fitness & 1.3578 & 0.0525 \\
\hline
\end{tabular}

Table 7.8 Results of the tool cutting condition monitoring 


\section{Chapter VIII}

\section{Introduction of MOGART Package}

Based on the analytical cutting force model and experimental data of micro-endmilling operations, a Micro-End-Milling Operation Guide and Research Tool (MOGART) package has been developed for the machinability studies, modeling and monitoring of micro-end-milling operations (see Figure 8.1).

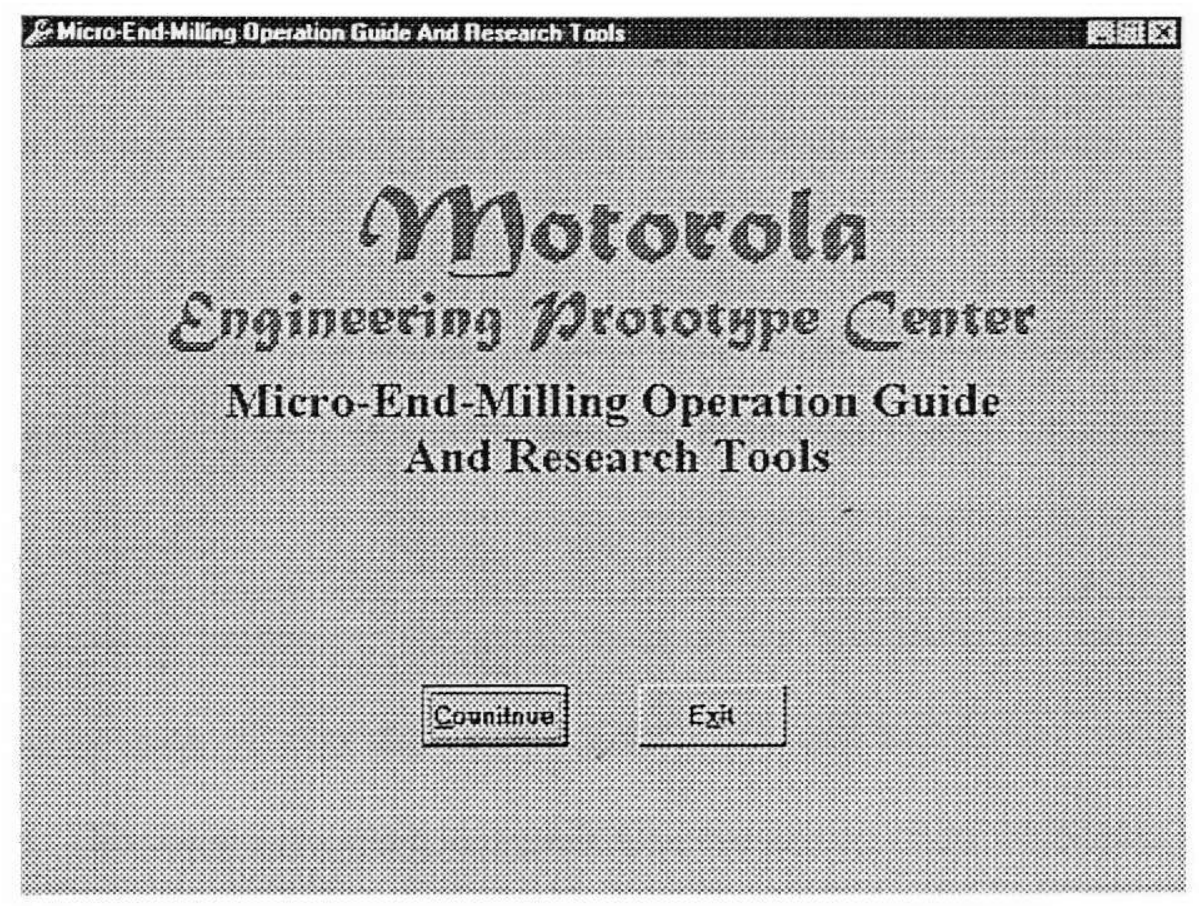

Figure 8.1 MOGART package

The package is capable to perform the following tasks by using a user friendly interface: 
- The model and neural network based cutting force estimation

- Cutting force characteristics analysis

- Investigation of machability

- Estimation of wear

- Detection of breakage

- Estimation of run-out

- Selection of optimal working conditions

- Estimation of surface roughness and precision

- Neural network based data mapping, forecasting and classification

- Genetic algorithm based modeling, monitoring and optimization

As a convenient and efficient research tool, the MOGART has being applied to the micro-end-milling studies of the Engineering Prototype Center of Radio Technology Division of Motorola Inc., which include machinability, wear, breakage, run-out and optimal working conditions. The performance of the MOGART has been tested on the experimental data of over 800 experimental cases and satisfactory results have been obtained. This chapter is a basic user guide for introduction of the MOGART program.

\subsection{Structure of MOGART Package}

The structure diagram of the MOGART program is presented in Figure 8.2 


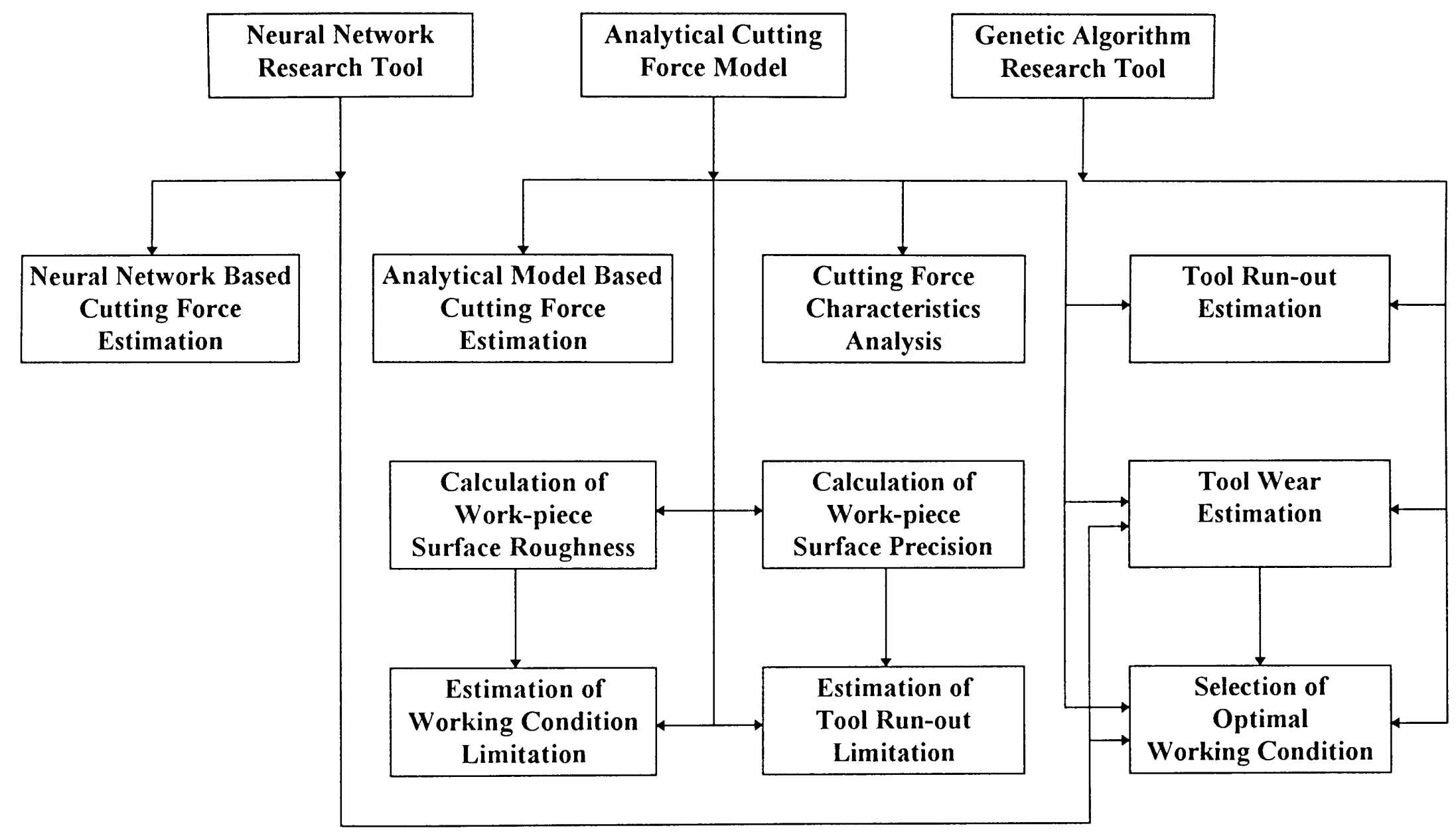

Figure 8.2 Diagram of MOGART program 
The MOGART includes three main research tools: the analytical cutting force model, neural networks and genetic algorithms. Also, it included many research applications: neural networks-based experimental data processing, analytical cutting force model-based machinability studies, cutting force estimation, work-piece surface precision and roughness calculation, genetic algorithms-based tool life, run-out and optimal working condition estimation.

The MOGART has four sections: the analytical cutting force model, neural network research tool, genetic algorithm research tool and operation guide applications of milling operations (see Figure 8.3). In the analytical cutting force model section, a tool to study the cutting force model of end milling operations is provided. The results of the studies are stored in the operation guide applications section. The neural network research tool can be used to generate empirical cutting force estimation models and wear forecasting models. The genetic algorithm research tool can be used to search the optimal parameters and coefficients of the analytical cutting force model. The neural network research tool (NNTool) and genetic algorithm research tool (GATool) are designed for general research purposes so that they can be used to solve data mapping, forecasting and optimization problems of other engineering systems.

The MOGART program is developed by using Visual Basic 4.0 version compiler. It has a friendly user interface that includes the data file import and Microsoft Excel Worksheet-based data input options, and visualized results that include the graphics of the tool cutting edge profiles, cutting force profiles and characteristics, and so forth. 
- For a new research project

1. From the File menu, choose Project.

2. From the Project sub-menu, select New Project.

3. In the New Project screen (see Figure 8.3), type the project directory and name, click Ok button.

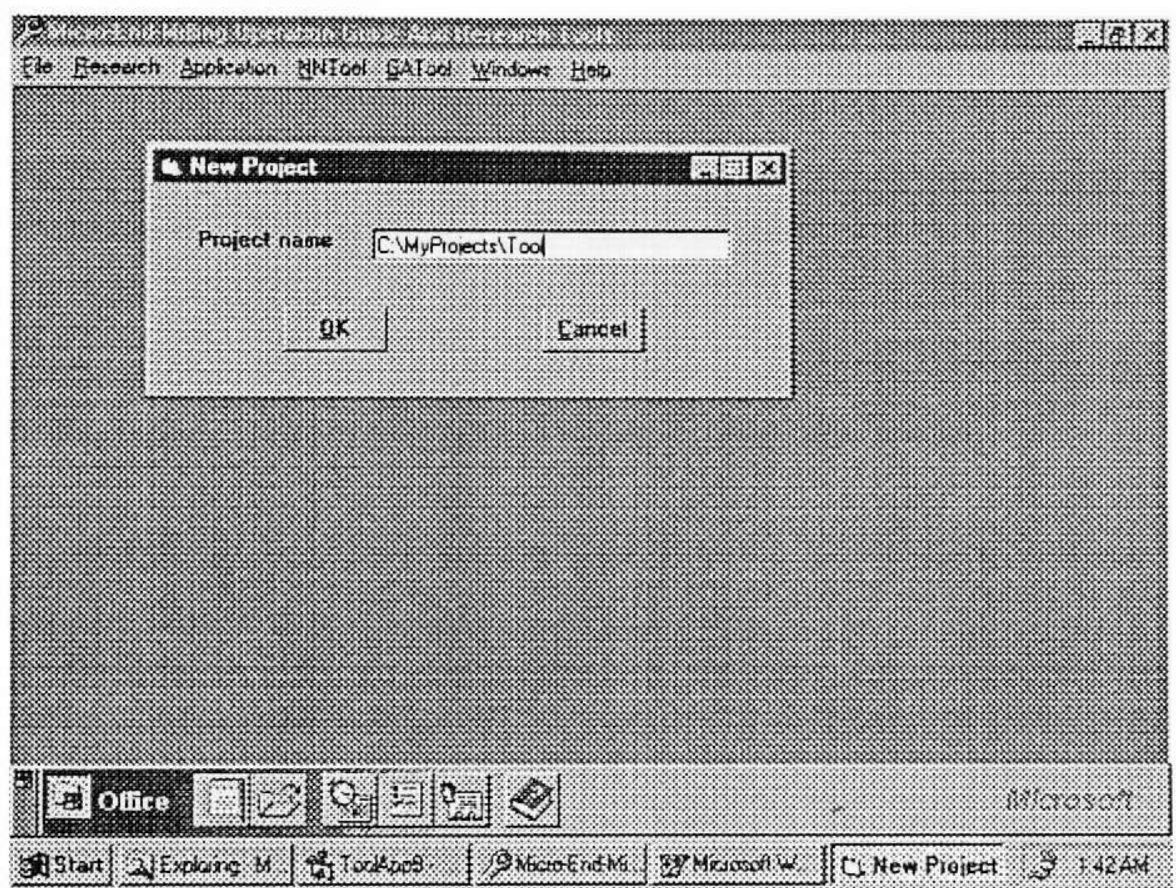

Figure 8.3 Menu of MOGART program

- For a matured project

1. From the File menu, choose Project.

2. From the Project sub-menu, select Tool Material.

3. From the Tool Material sub-menu, select tool material.

4. From the Project sub-menu, select Work-piece Material.

5. From the Work-piece Material sub-menu, select work-piece material. 


\subsection{Analytical Cutting Force Model}

Based on the analytical cutting force model, the MOGART can be used to calculate the tool cutting forces of milling operations by the formulas derived in the model. There are four features, Cutting Force Estimation, Cutting Force Report, Cutting Force Graphic and Tool Cutter Profile, which are included in the main menu Research section.

- To estimate the tool maximum and minimum cutting forces in the feed and thrust directions

1. From the Research menu, choose Cutting Force Estimation.

2. In the Cutting Force Estimation screen (see Figure 8.4), there are three tool geometry parameters, seven tool working condition parameters and two material coefficients. Select the parameters and coefficients one by one by pointing the cursor at the text box and click the right mouse button, and type the data depending on the tool operation conditions. For example, if there is a $50 \%$ overlapped climbing milling case, type 90 in the Cutting Start Angle text box and 180 in the Cutting End Angle text box.

3. One option is the calculation precision angle. 5 degrees is chosen as the default calculation angle, which means the tool cutting force data are calculated at every 5 degrees of the cutting period. The maximum calculation precision can be selected to 1 degree.

4. Click Ok button. 


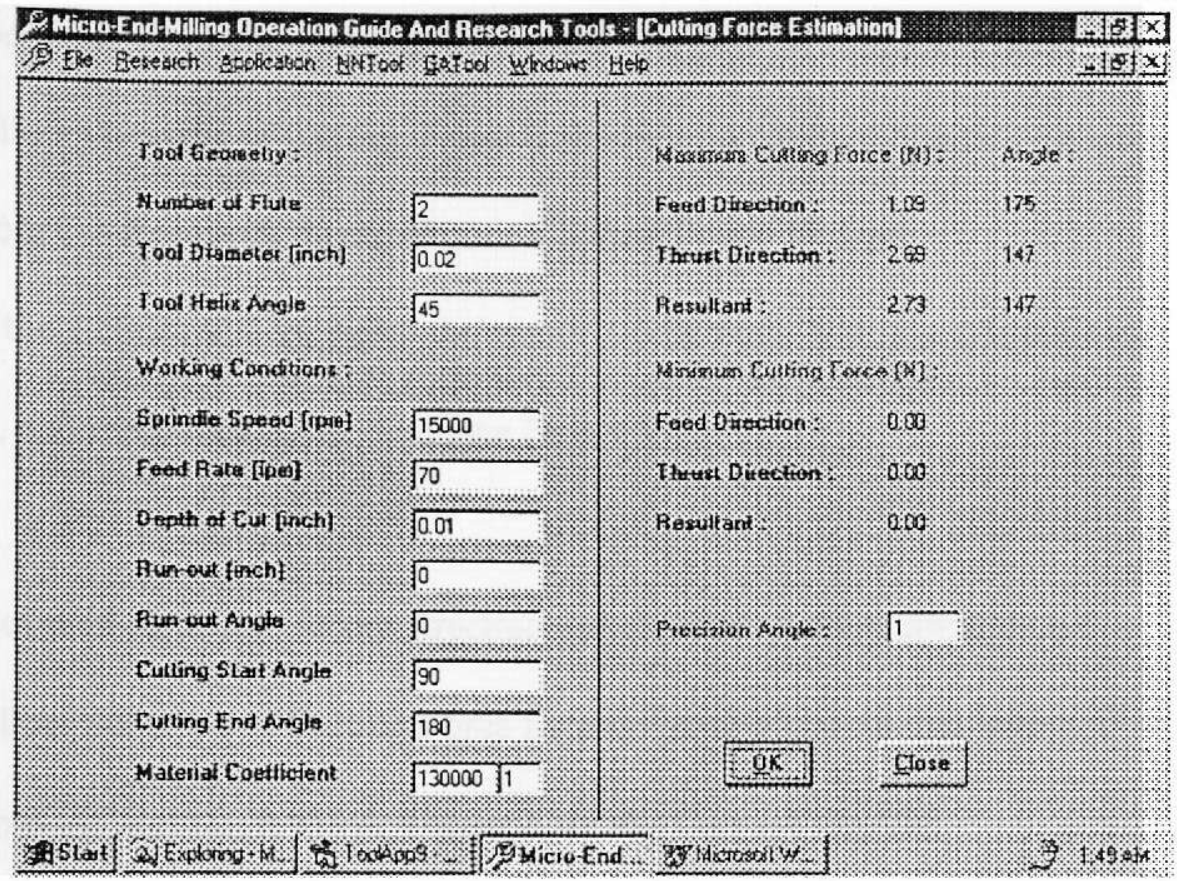

Figure 8.4 Cutting force estimation

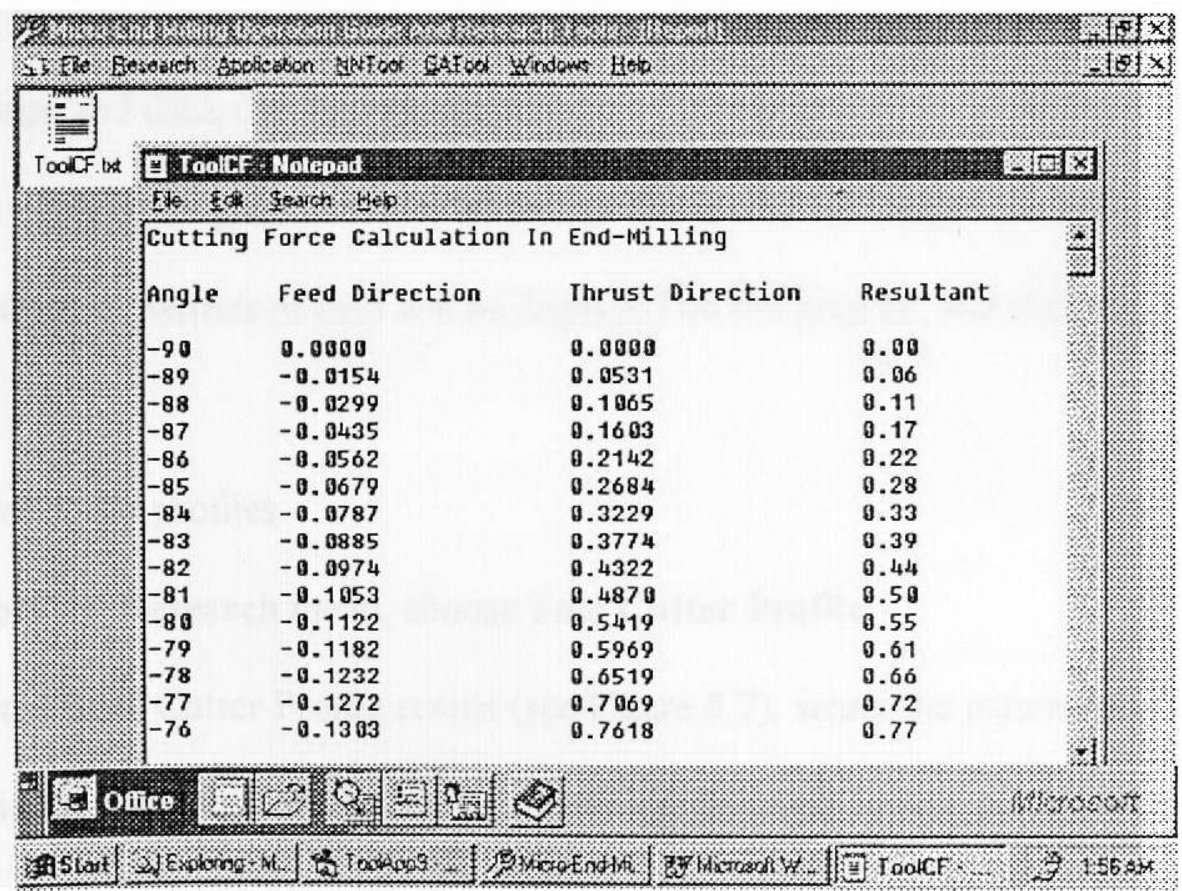

Figure 8.5 Cutting force report 
- Tool feed direction, thrust direction and resultant cutting force profile estimation data

1. Follow the steps of Cutting Force Estimation to estimate the cutting force.

2. From the Research menu, choose Cutting Force Report.

3. In the Report screen, point the cursor to the icon with the project name you worked on, and double-click the icon (see Figure 8.5).

- Tool feed direction, thrust direction and resultant cutting force graphics

1. Follow the steps of Cutting Force Estimation to estimate the cutting force.

2. From the Research menu, choose Cutting Force Graphic.

3. In the Cutting Force Graphic screen (see Figure 8.6), point the cursor to the check box and click the right mouse button to check the force graphic you would like to see.

4. To change the tool turning angle display option to time, point the cursor to the option button and click the right mouse button.

5. To change the graphic scale, select the Scale text box, type the scale, which decides how many periods of data will be displayed on the graphic, and click Ok button.

- Tool cutter profiles

1. From the Research menu, choose Tool Cutter Profile.

2. In the Tool Cutter Profile screen (see Figure 8.7), select the parameters one by one by pointing the cursor at the text box and click the right mouse button, and type the data depending on the tool operation conditions.

3. Click Ok button. 


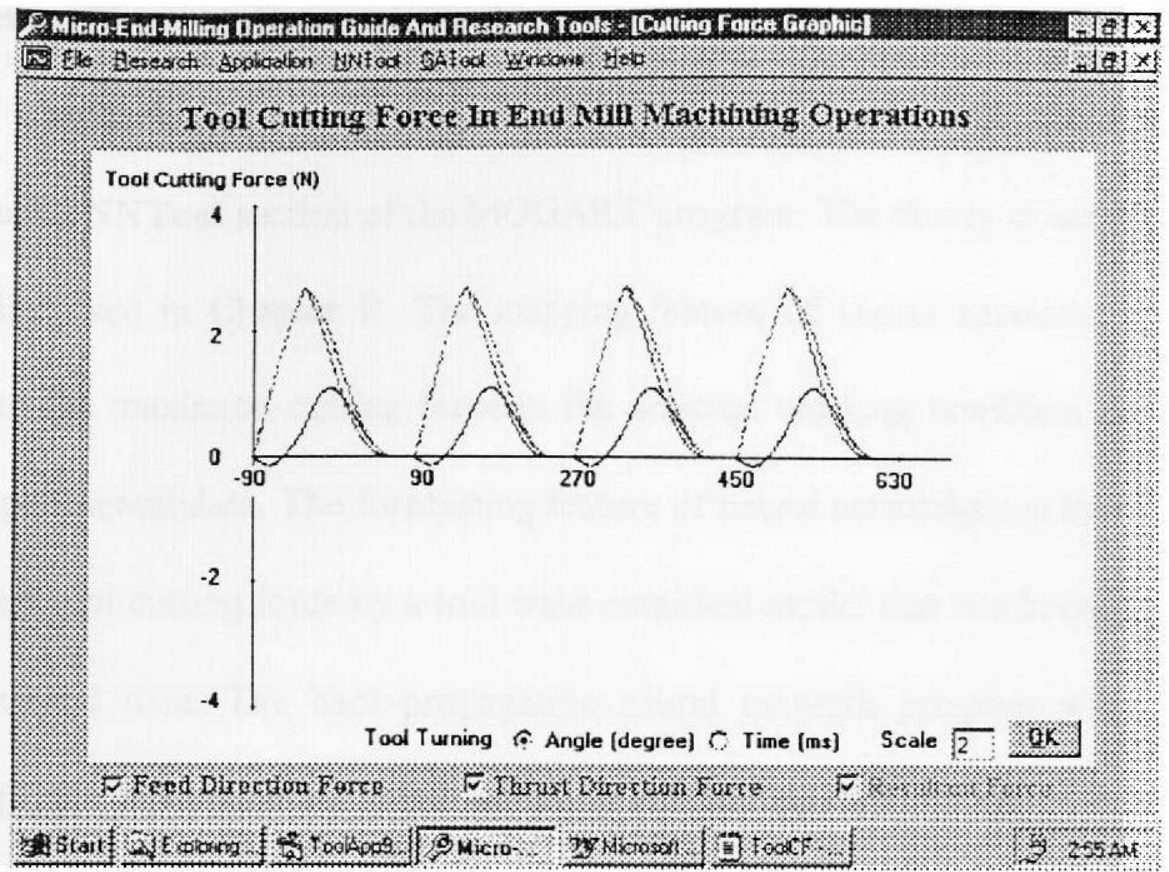

Figure 8.6 Cutting force profile

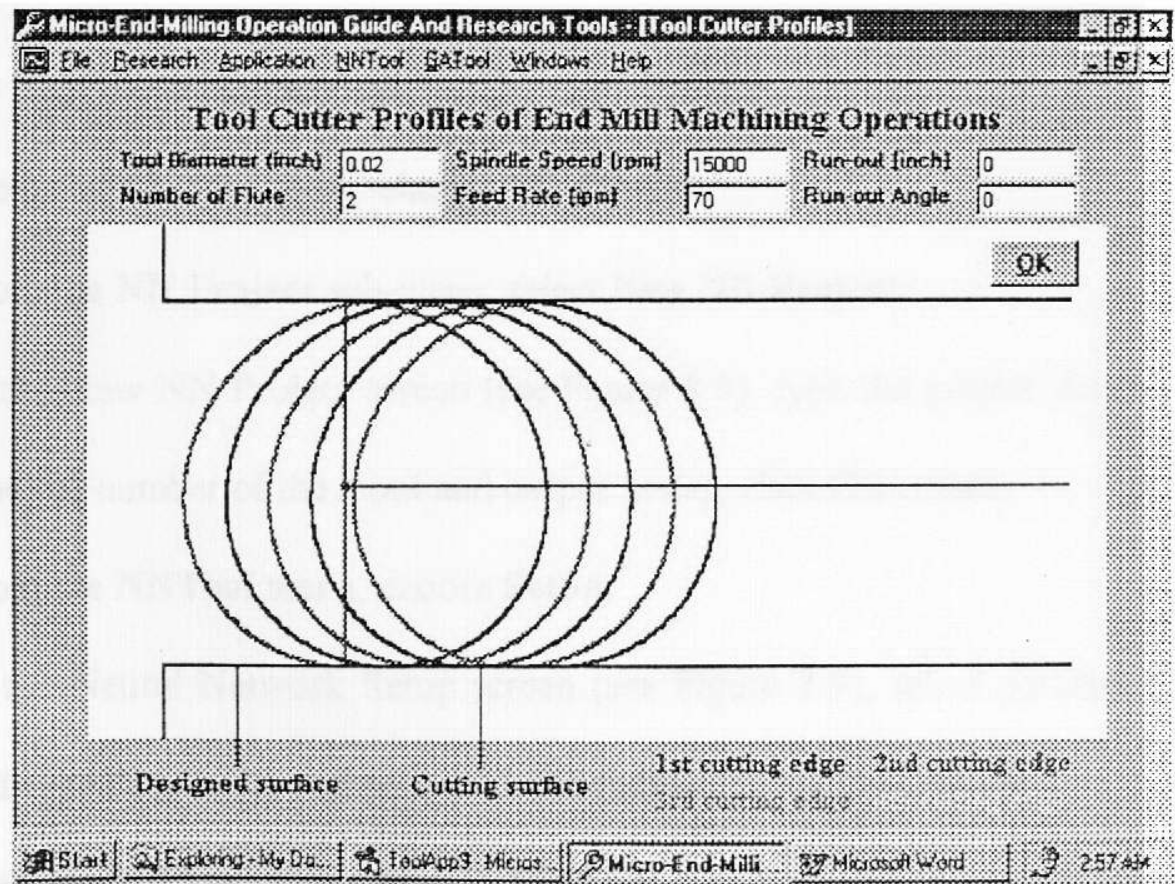

Figure 8.7 Tool cutter profile 


\subsection{Neural Network Research Tool}

The back-propagation neural network as a data processing tool is included in the main menu NNTool section of the MOGART program. The theory of neural networks has been discussed in Chapter II. The mapping feature of neural networks can be used to estimate the maximum cutting force in the selected working condition ranges by only a few experimental data. The forecasting feature of neural networks can be used to estimate the worn tool cutting force by a tool wear empirical model that has been generated by the experimental data. The back-propagation neural network program was developed for general data processing purposes so that it can be used for studies of micro-end-milling operation and other engineering systems.

The NNTool includes three sections, Training, Testing and Results.

- To train the neural network

1. From the NNTool menu, choose NN Project.

2. From the NN Project sub-menu, select New NN Project.

3. In the New NN Project screen (see Figure 8.8), type the project directory and name, type the number of the input and output nodes, click Ok button.

4. From the NNTool menu, choose Setup.

5. In the Neural Network Setup screen (see Figure 8.9), select parameters one by one and type data in the text box, click Ok button.

6. From the NNTool menu, choose Training. 


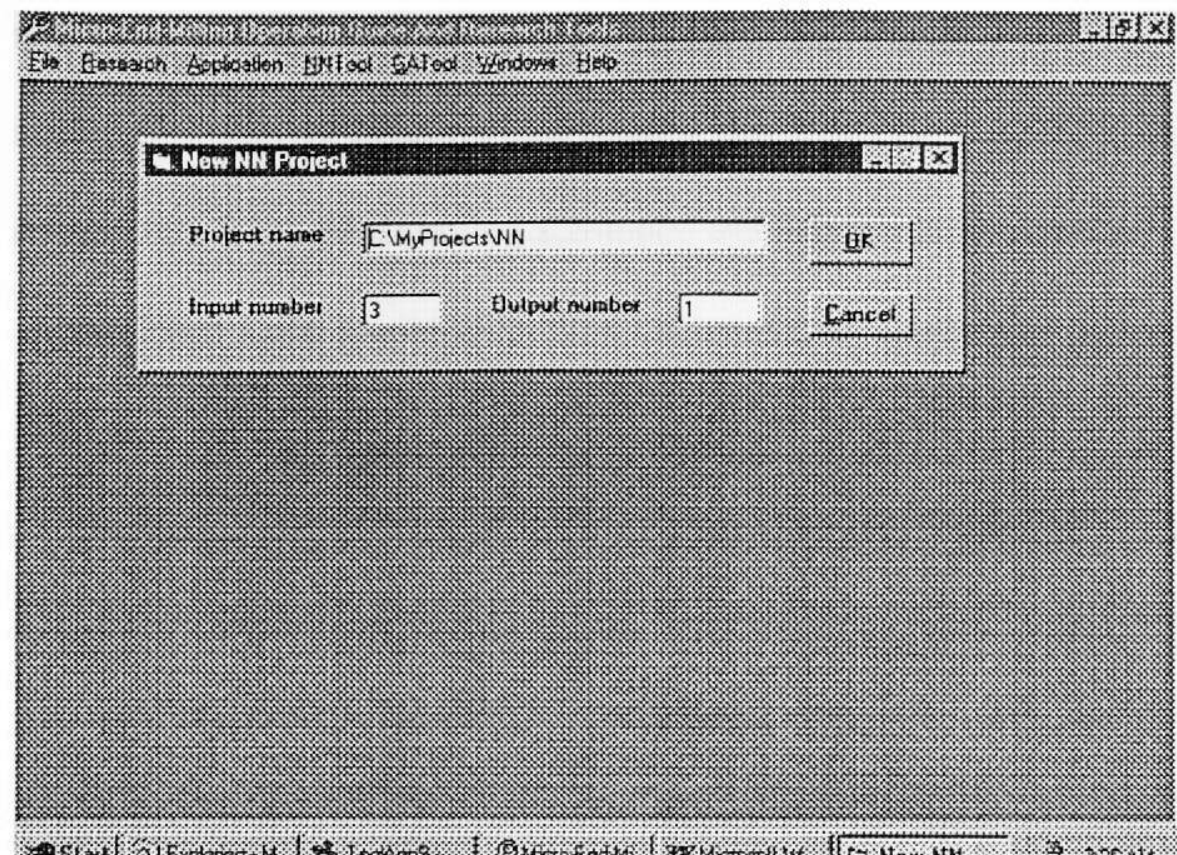

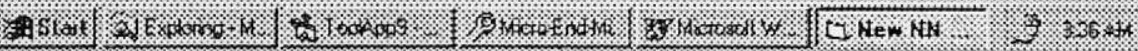

Figure 8.8 Neural network project

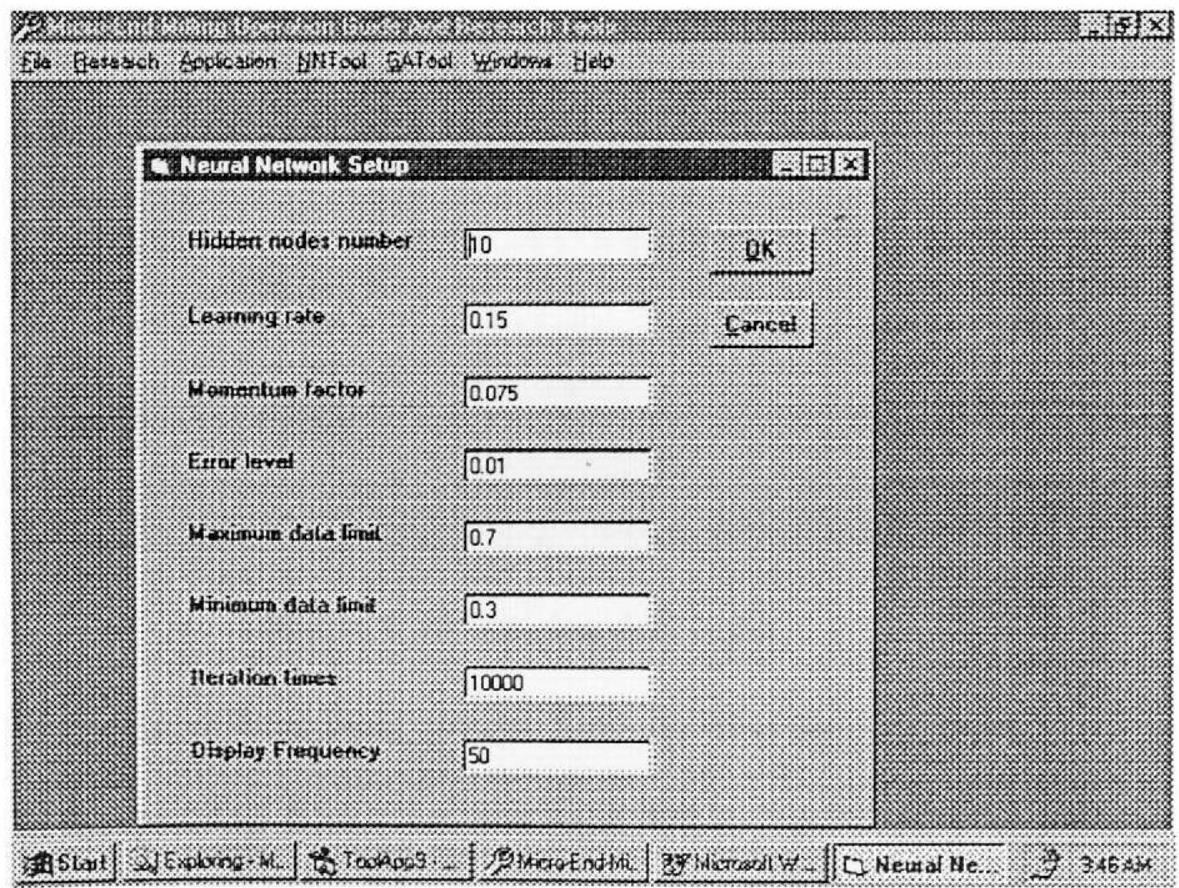

Figure 8.9 Neural network setup 
7. Import a pre-prepared training data file by selecting Import Training File from the Training sub-menu, or input training data on the Excel Worksheet by selecting Input Training Data from the Training sub-menu and follow the steps below:

(1) In the Input Data screen, point the cursor at the upper-right corner and double click it.

(2) In the Excel Worksheet (see Figure 8.10), type the number of the training data cases; type the input data then output data one case by one case; type the support service phone number 3053483304 as a data checking number.

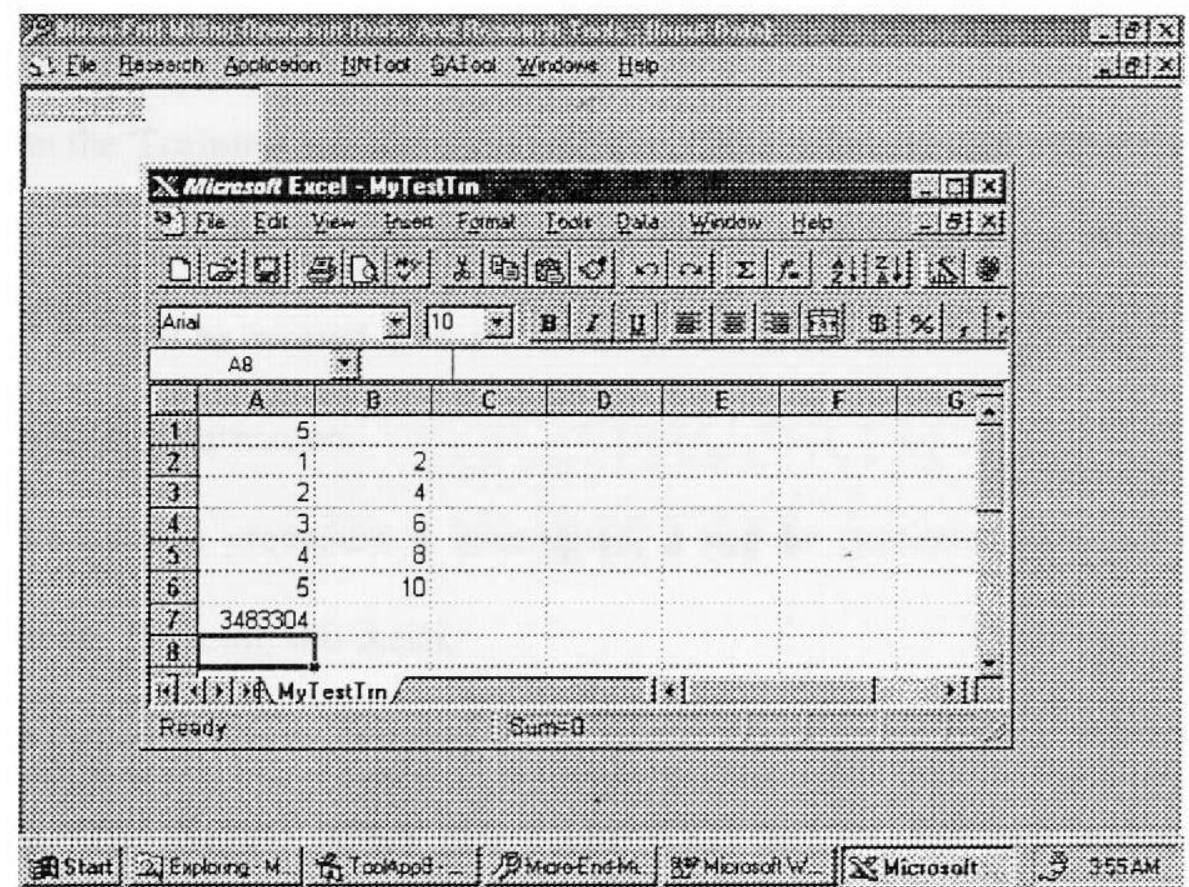

Figure 8.10 Training data input

(3) In the Excel Worksheet, choose Save As from the File menu.

(4) In the Save As screen, select Text (OS/2 or MS-DOS) (*.txt) from Save As type section. Find the project directory, and click Save button to save the training data file with name "project name + Trn.txt." 
(5) Close the Excel Worksheet.

8. From the Training sub-menu, select Setup Start Point.

9. From the Setup Start Point sub-menu, select Random, Index Random or Constant. If selecting Random, it will set a random start point. If selecting Index Random, type a random index number in the Random Start Point screen and click Ok button. It will set a random start point depending on the given index number so that the running procedure can be repeated. If selecting Constant, type a constant number in the Constant Start Point screen and click Ok button. It will set all connection weights as a given constant at the beginning.

10. From the Training sub-menu, select Start. The neural network will start to be trained until it reaches the given Error Level or maximum Iteration times. A "project name + .prj" file will be created after the training, which includes all the neural network data processing information.

11. If the training procedure is interrupted, it can be continued by selecting Continue from the Training sub-menu.

12. One option is Show Running Error in the Training sub-menu. If it is checked, the Data Train Report screen (see Figure 8.11) will be displayed during the training procedure.

- To test the neural network

1. After training, choose NN Project from the NNTool menu.

2. From the NN Project sub-menu, select Import NN Project (see Figure 8.12).

3. In the Import Project screen, find the project directory and name, double click it. 


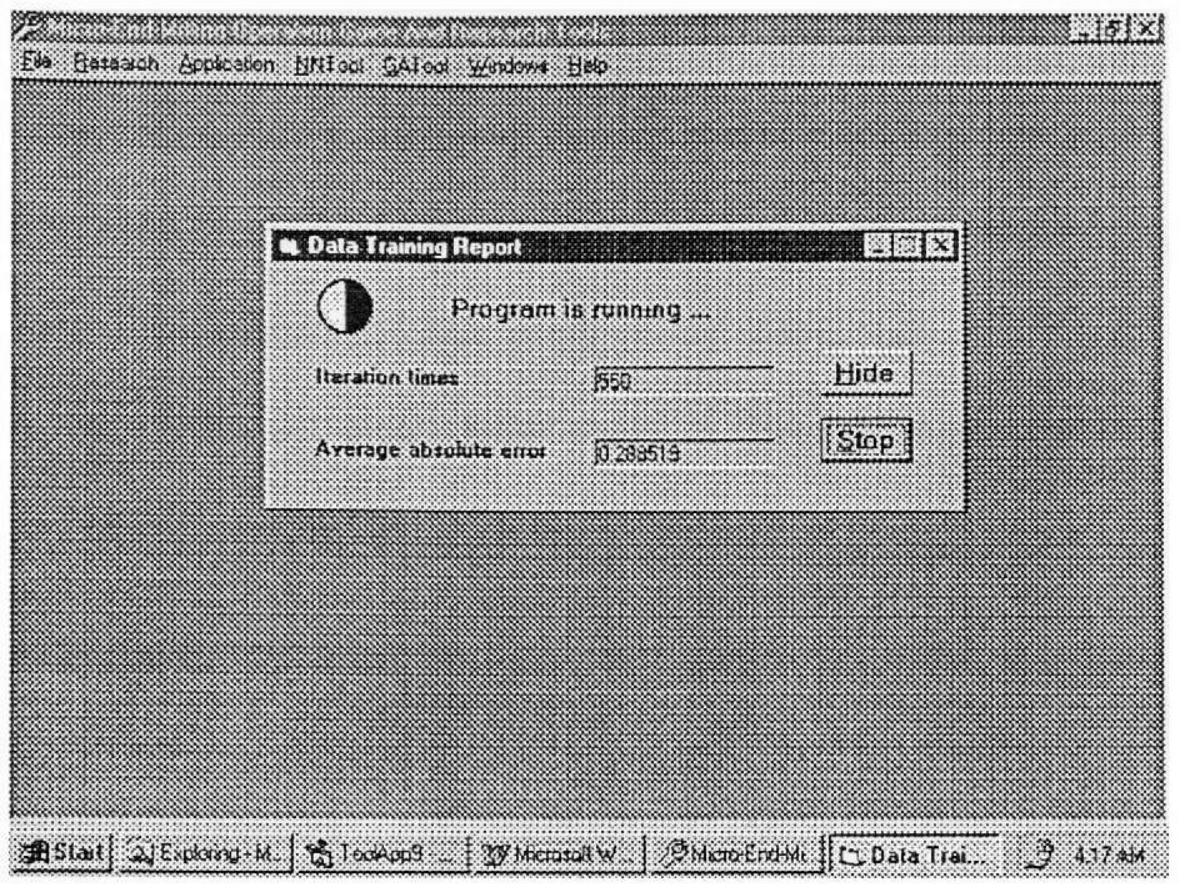

Figure 8.11 Neural network running

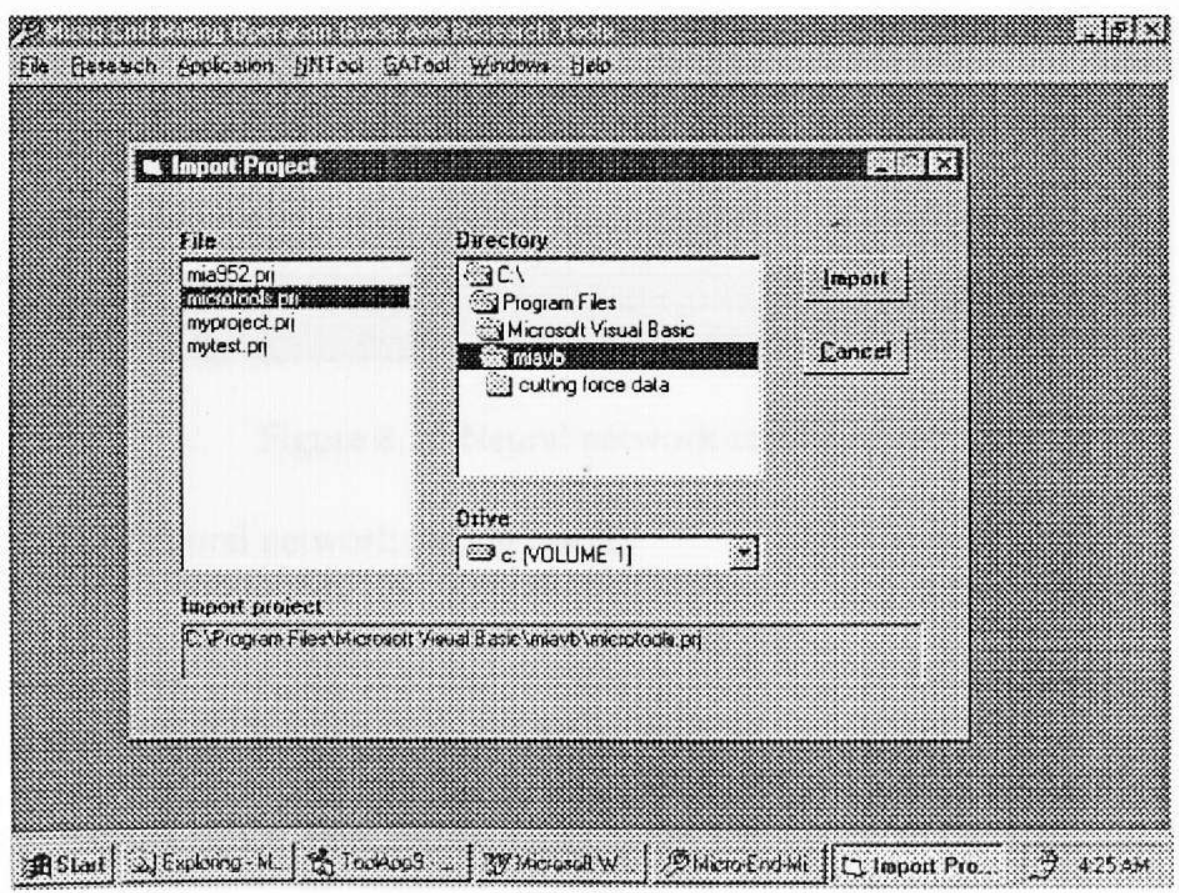

Figure 8.12 Import the project 
4. From the NNTool menu, choose Testing.

5. From the Testing sub-menu, select Import Testing File from the Testing sub-menu, or input testing data on the Excel Worksheet by selecting Input Testing Data from the Testing sub-menu and follow step 7 of the training section.

6. From the Testing sub-menu, select Start. A "project name + Rpt.txt" file will be created after the testing.

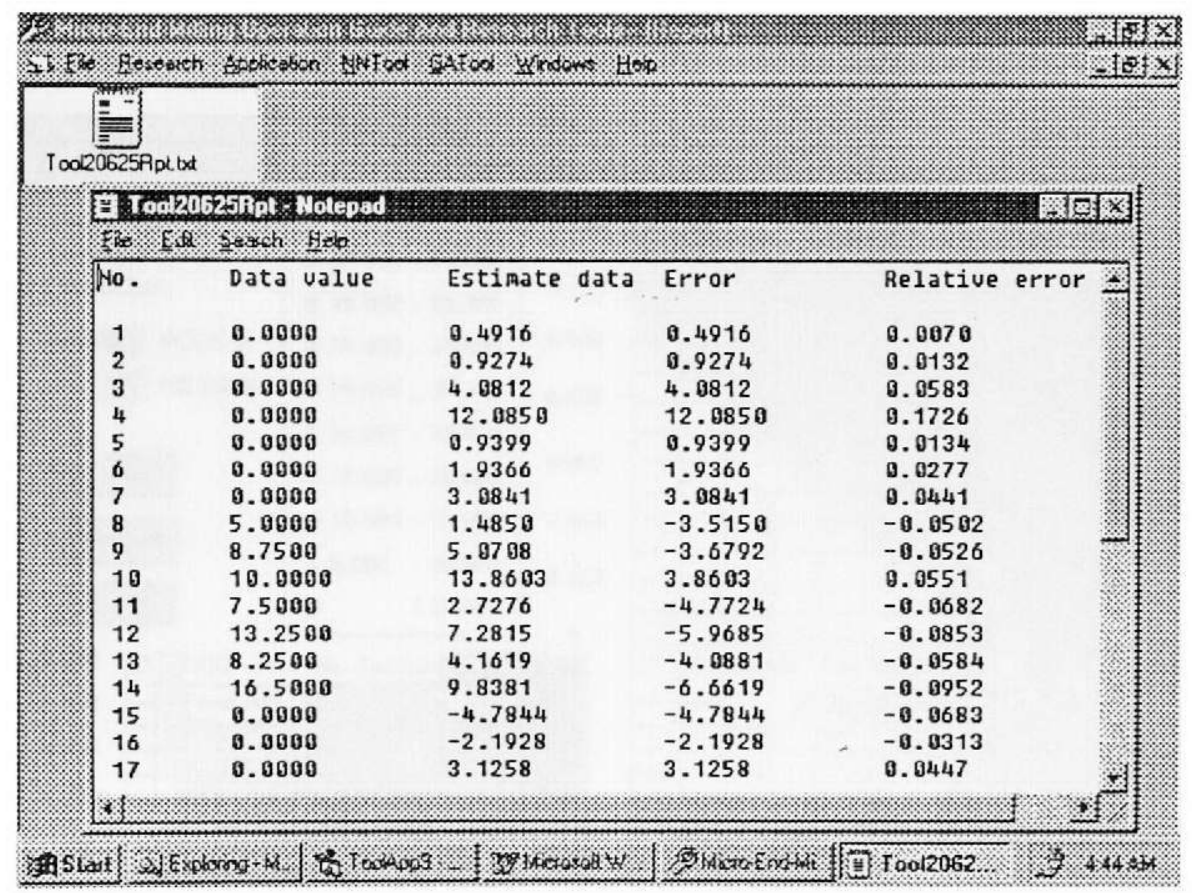

Figure 8.13 Neural network testing report

- The data of neural network testing results

1. After testing, choose Results from the NNTool menu.

2. From the Results sub-menu, select Show Report.

3. In the Report screen (see Figure 8.13), point the cursor to the icon that has a project name you worked on, and double click the icon. 
- The graphics of neural network testing results

1. After testing, choose Results from the NNTool menu.

2. From the Results sub-menu, select Show Graphic.

3. In the 3 Dimension Cutting Force Distribution screen (see Figure 8.14), click the option button, type the working condition data in the gray area, and click the Redraw button. It will redraw a given case's graphics.

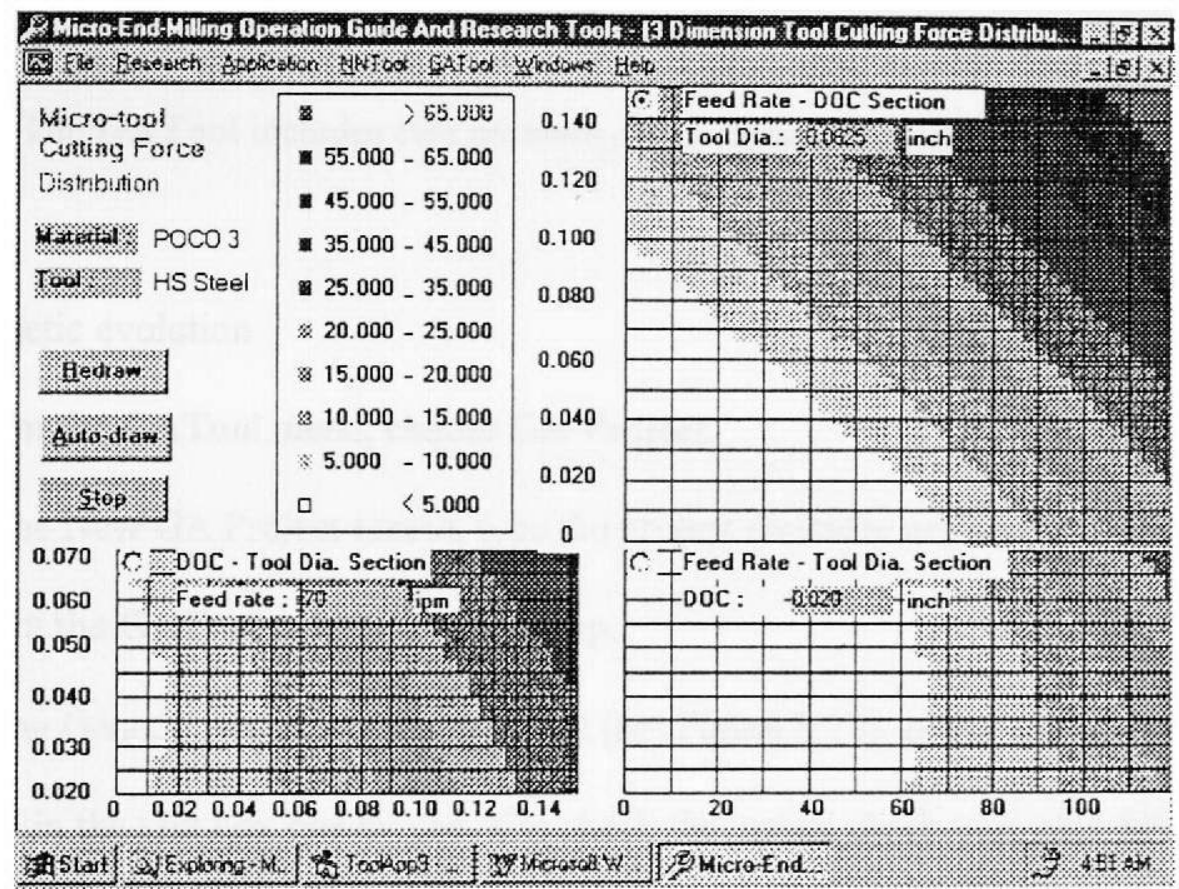

Figure 8.14 Graphics of neural network testing results

4. One option is to click the option button, and click Auto-draw button. It will draw the graphic layer by layer automatically.

5. Click Stop button to stop the Auto-draw function. 


\subsection{Genetic Algorithm Research Tool}

The genetic algorithm as a data analysis tool is included in the main menu GATool section of the MOGART program. The theory of the genetic algorithms has been discussed in Chapter II. The optimization feature of genetic algorithms can be used to monitor the tool wear, run-out, cutting angles and working conditions with the analytical cutting force model. The genetic algorithm was developed for general optimization purposes so that it can be used for monitoring micro-end-milling operations and solving optimization problems of other engineering systems.

The GATool includes two sections, Generator and Results.

- Genetic evolution

1. From the GATool menu, choose GA Project.

2. In the New GA Project screen, type the project directory and name, click Ok button.

3. From the GATool menu, choose Setup.

4. In the Genetic Algorithm Setup screen (see Figure 8.15), select parameter and type the data in the text box one by one, also check the option check box, click Ok button.

5. In another Genetic Algorithm Setup screen (see Figure 8.16), select parameter and type the data range and the number of chromosomes in the text box one by one, click Ok button.

6. From the GATool menu, choose Generator.

7. From the Generator sub-menu, select Setup Start Point. 


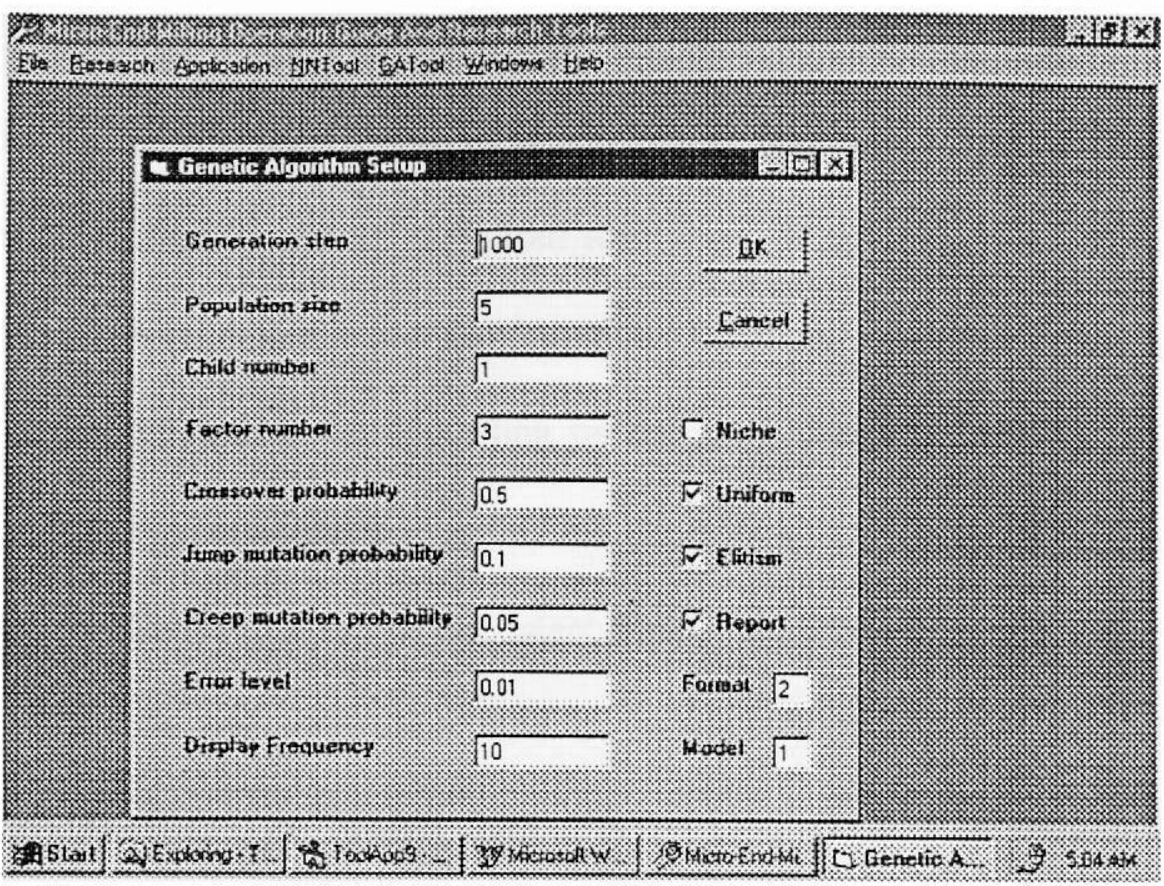

Figure 8.15 GATool setup step 1

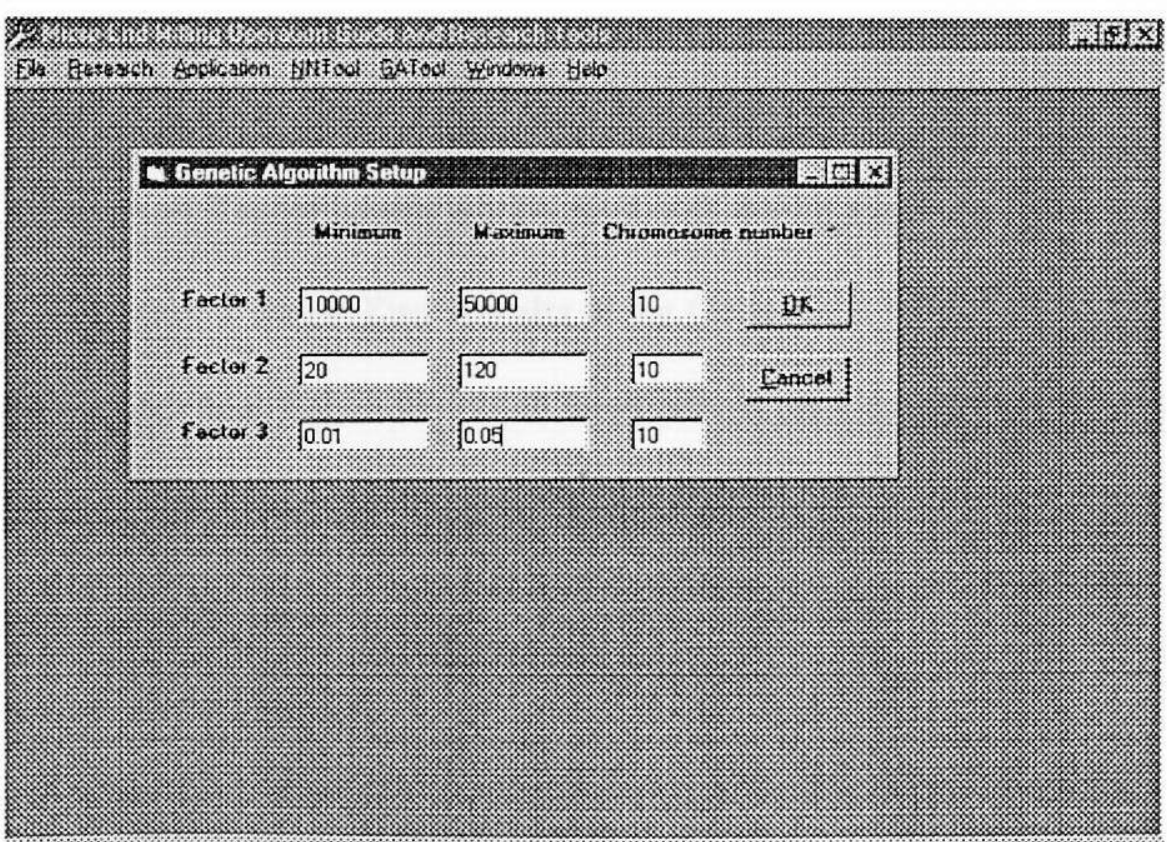

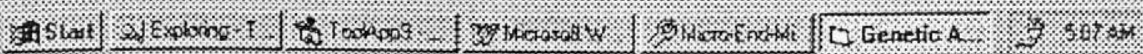

Figure 8.16 GATool setup step 2 
8. From the Setup Start Point sub-menu, select Random or Index Random. If you select Random, it will set a random start point. If selecting Index Random, type a random index number in the Random Start Point screen and click Ok button. It will set a random start point depending on the given index number so that the running procedure can be repeated.

9. From the Generator sub-menu, select Start. The Generator will start to create one generation by one generation until it reaches the given Error Level or maximum Generation Steps. A "project name + Report.txt" file will be created after running, which includes all the generation data information.

10. If the running process is interrupted, it can be continued by selecting Continue from the Generator sub-menu.

11. One option is Show Running Fitness in the Generator sub-menu. If it is checked, the Generation Report screen will be displayed during the running process.

12. Another option is Show End Results in the Generator sub-menu. If it is checked, the Results screen (see Figure 8.17) will be displayed after the running process. It tells the best fitness and factor data in the last generation.

- Genetic evolution results

1. After testing, choose Results from the GATool menu.

2. In the Report screen, point the cursor to the icon that has a project name you worked on, and double click the icon. 


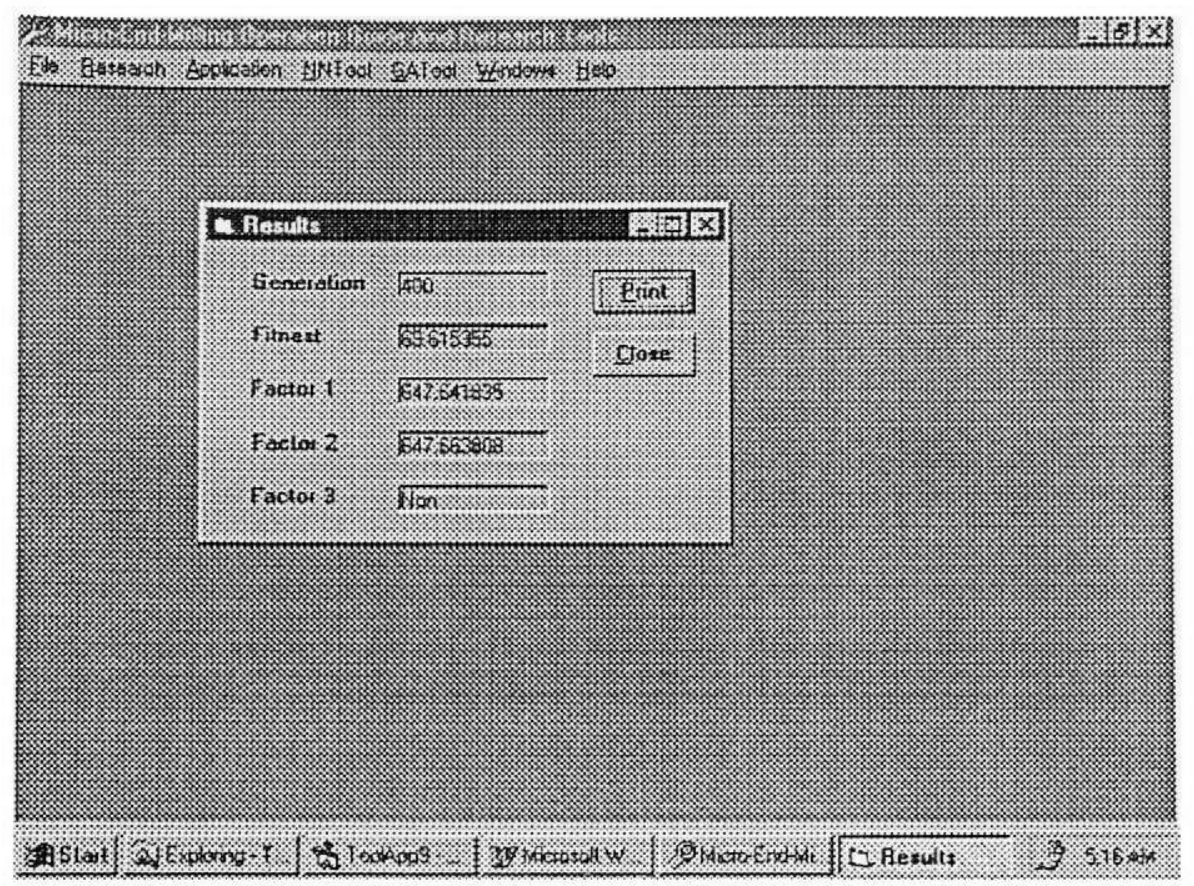

Figure 8.17 GATool results

\subsection{Applications of Operation Guide in Micro-End-Milling Operations}

Based on the analytical cutting force model, six tool operation guide applications have been done, Cutting Force Estimate, Work-piece Surface Precision Estimation, Work-piece Surface Roughness Estimation, Tool Run-out Estimation, Tool Life Estimation and Working Condition Selection, which are included in the main menu Application section.

- To estimate the tool feed direction and thrust direction maximum cutting force characteristics with different parameters

1. From the Application menu, choose Cutting Force Estimate. 


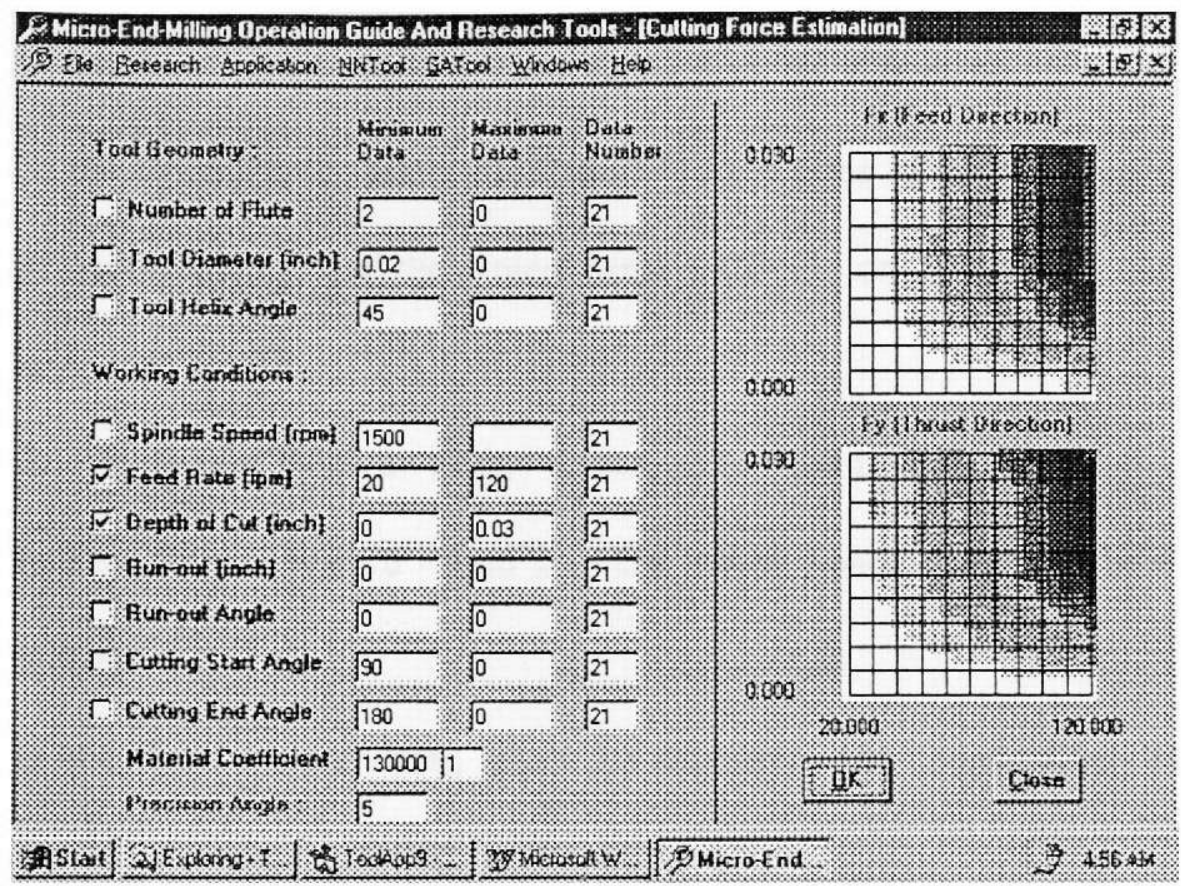

Figure 8.18 Characteristics of maximum cutting forces

2. In the Cutting Force Estimation screen (see Figure 8.18), select one or two parameters by pointing the cursor at the check box and click the right mouse button, type the data range in the Maximum and Minimum Data text box, and the data number in the Data Number text box.

3. Click Ok button.

4. To know the cutting force data, point the cursor to the Fx (Feed Direction) or Fy (Thrust Direction) and click the right mouse button.

5. To have a nice hard copy of the graphics, follow steps as below:

(1) In the Cutting Force Estimation screen, double click the graphic.

(2) In the Report screen, point the cursor at the upper-right corner and double click it. 
(3) In the Excel sample sheet, choose Open from the File menu.

(4) In the Open screen, select All files $\left({ }^{*} .^{*}\right)$ from Files of the type section. Under the project directory, find the project name + CFx.txt or CFy.txt file, and double click it to open the file.

(5) In Text Import Wizard - Step 1 screen, click Next button.

(6) In Text Import Wizard - Step 2 screen, check Space check box, and click Finish button.

(7) In the Excel sample sheet, select the data area, and choose Copy from the Edit menu.

(8) In the Excel sample sheet, choose ToolCF.xls from the Window menu.

(9) In the Excel sample sheet, if it is one parameter, select the CF2Ddata sheet; or if it is two parameters, select the CF3Ddata sheet.

(10) In the CF2Ddata sheet or CF3Ddata sheet, select the same data area, and choose

Paste from the Edit menu.

(11) Click CF2Dchart to see maximum cutting force with one parameter graphic or click CF3Dchart1 and CF3Dchart2 to see the maximum cutting force with two parameter graphics (see Figure 8.19).

(12) To get a hard copy, choose Print from the File menu. 


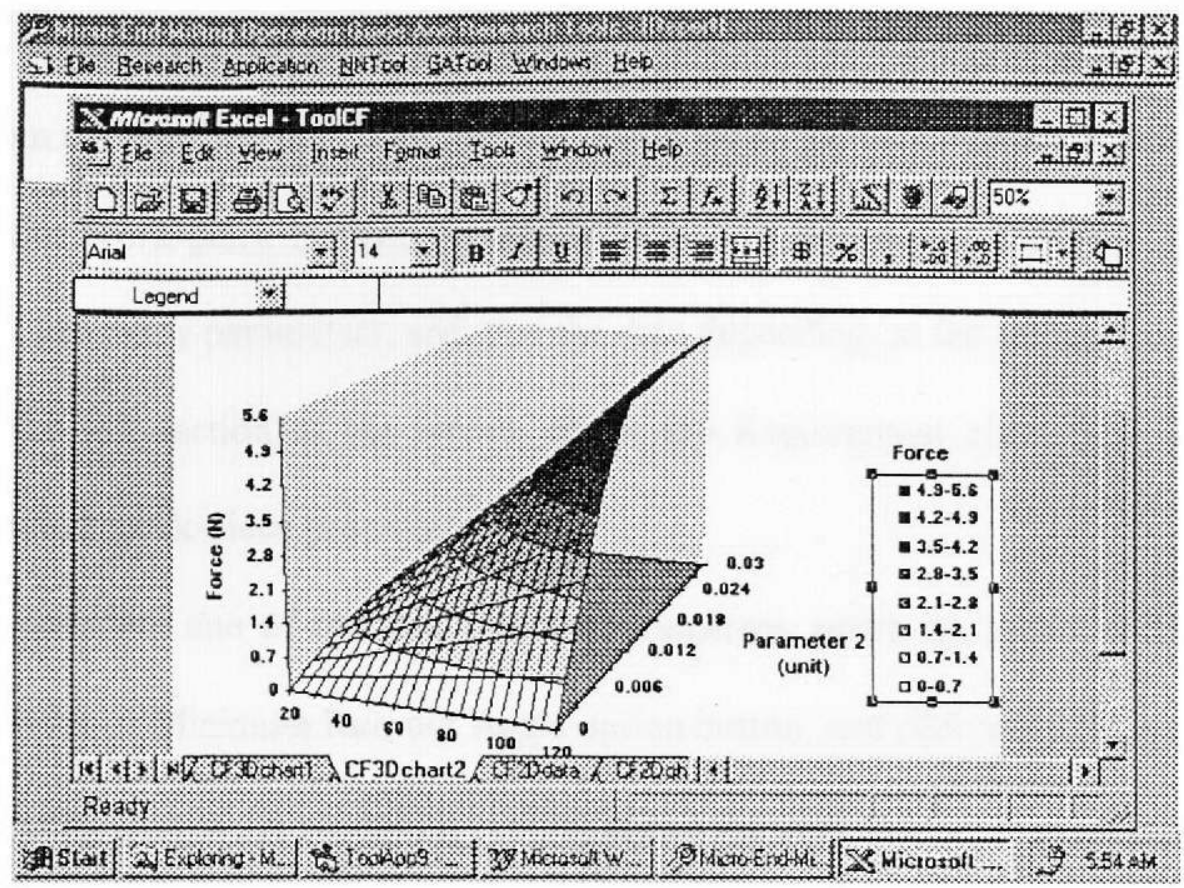

Figure 8.19 Three-dimensional cutting force graphic

- To estimate the work-piece surface precision depending on the tool run-out

1. From the Application menu, choose Work-piece Surface Precision Estimation.

2. In the Work-piece Surface Precision Estimation screen (see Figure 8.20), select the tool geometry parameters by pointing the cursor at the text box and click the right mouse button, and type the data depended on the tool operation case.

3. In the right section of the screen, check the Working Condition check box, and select the run-out parameters by pointing the cursor at the text box and click the right mouse button, and type the data depending on the tool operation case.

4. Click Ok button. 
- To estimate the tool run-out depending on the work-piece surface precision

1. From the Application menu, choose Work-piece Surface Precision Estimation.

2. In the Work-piece Surface Precision Estimation screen (see Figure 8.20), select the tool geometry parameters, and type the data depending on the tool operation case.

3. In the left section of the screen, check the Requirement check box, and type the required work-piece precision.

4. To calculate one of the tool run-out parameters, point the cursor to the Maximum Run-out or Minimum Run-out Angle option button, and click the right mouse button.

5. Type the data in the other tool run-out parameter text box.

6. Click Ok button.

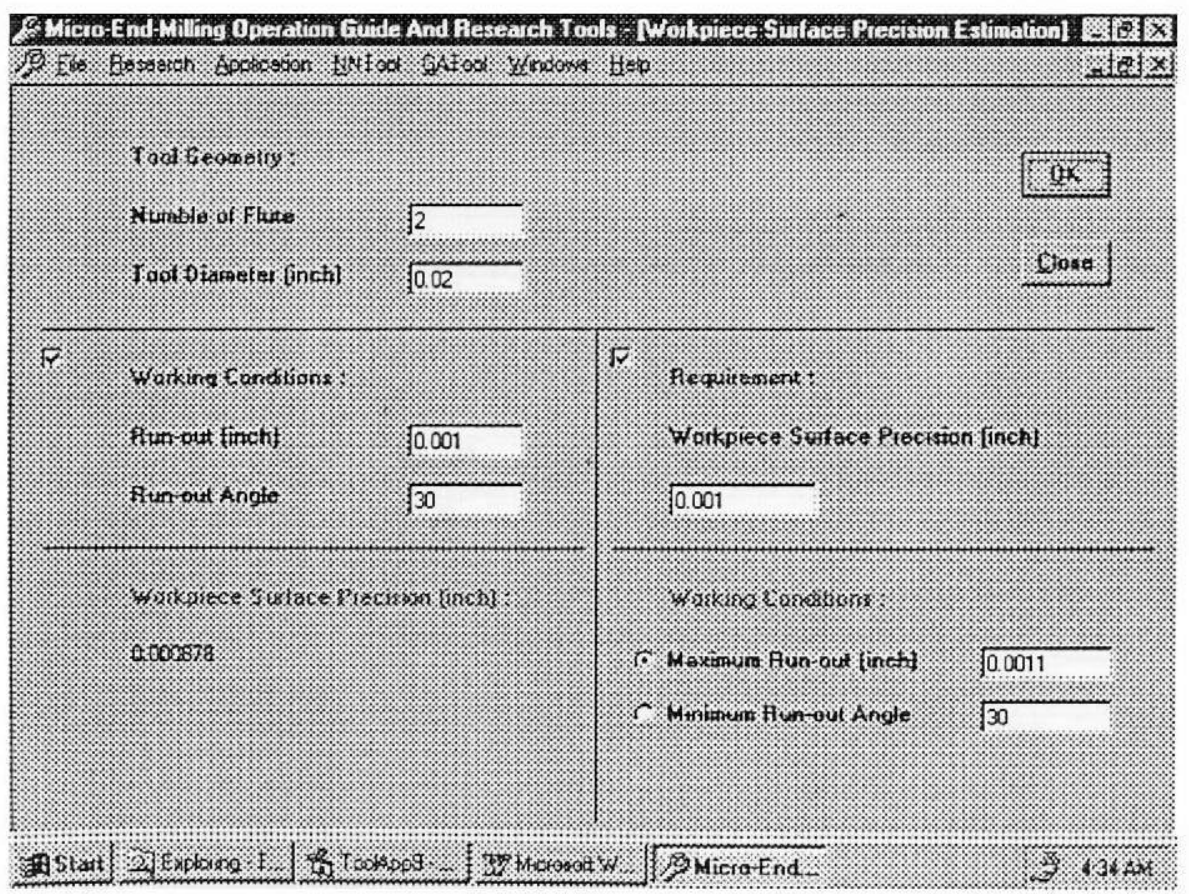

Figure 8.20 Work-piece surface precision estimation 
- To estimate the work-piece surface roughness depending on the tool cutting edge tip profiles

1. From the Application menu, choose Work-piece Surface Roughness Estimation.

2. In the Work-piece Surface Roughness Estimation screen (see Figure 8.21), select the tool geometry parameters, and type the data depending on the tool operation case.

3. Under the Milling Type section, check the Conventional Milling or Climbing Milling check box.

4. In the right section of the screen, check the Working Condition check box, and select working condition parameters, and type the data depending on the tool operation case.

5. Click Ok button.

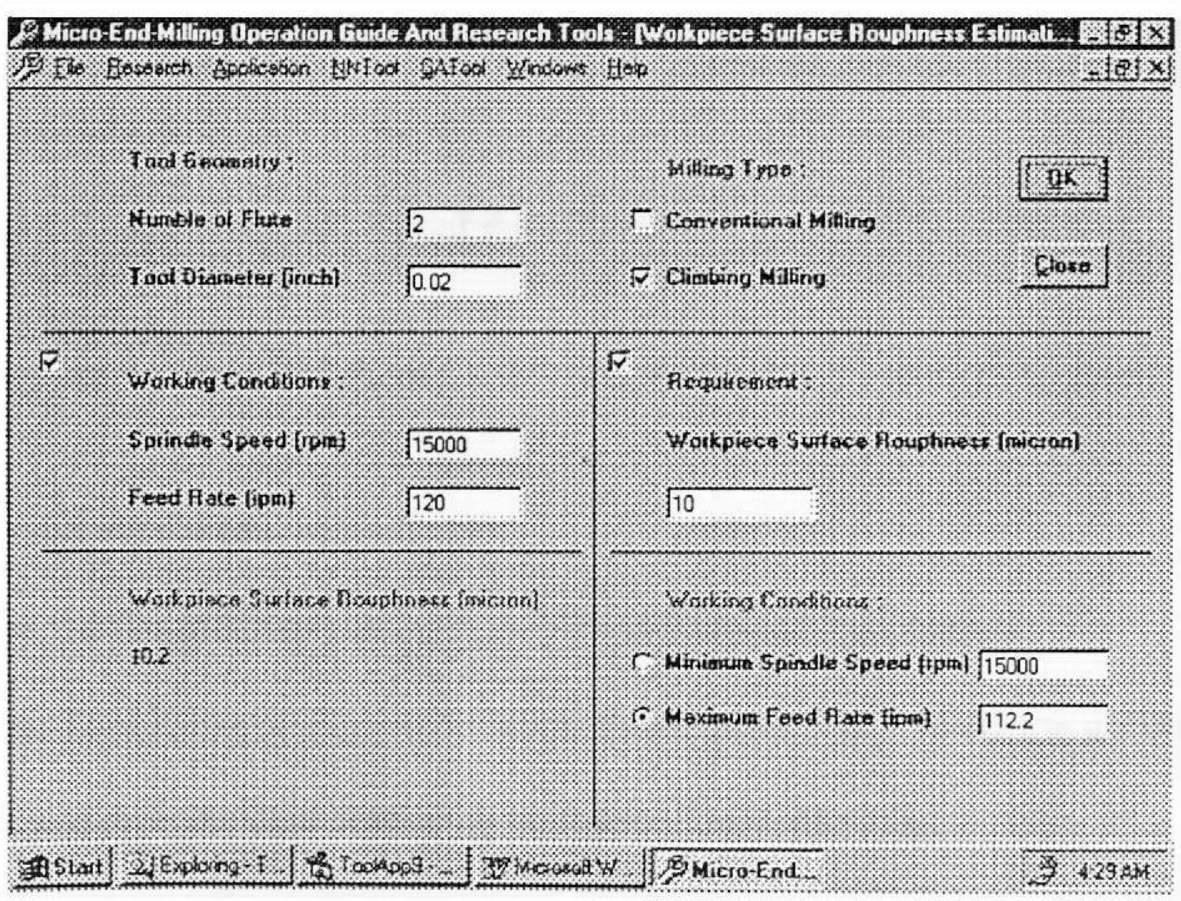

Figure 8.21 Work-piece surface roughness estimation 
- To estimate the tool working condition depending on the work-piece surface roughness

1. From the Application menu, choose Work-piece Surface Roughness Estimation.

2. In the Work-piece Surface Roughness Estimation screen (see Figure 8.21), select the tool geometry parameters, and type the data depending on the tool operation case.

3. Under the Milling Type section, check the Conventional Milling or Climbing Milling check box.

4. In the left section of the screen, check the Requirement check box, and type the required work-piece surface roughness.

5. To calculate one of the working condition parameters, point the cursor to the Minimum Spindle Speed or Maximum Feed Rate option button, and click the right mouse button.

6. Type the data in the text box of the other working condition parameters.

7. Click Ok button.

- To estimate the tool run-out

1. From the Application menu, choose Tool Run-out Estimation.

2. In the Tool Run-out Estimation screen (see Figure 8.22), select the parameters one by one, and type the data depending on the tool operation case. 
3. In the left section of the screen, type the experimental data of the maximum cutting force in feed direction and thrust direction. The largest one of the two flutes is defined as the $1^{\text {st }}$ cutting edge.

4. Click Ok button.

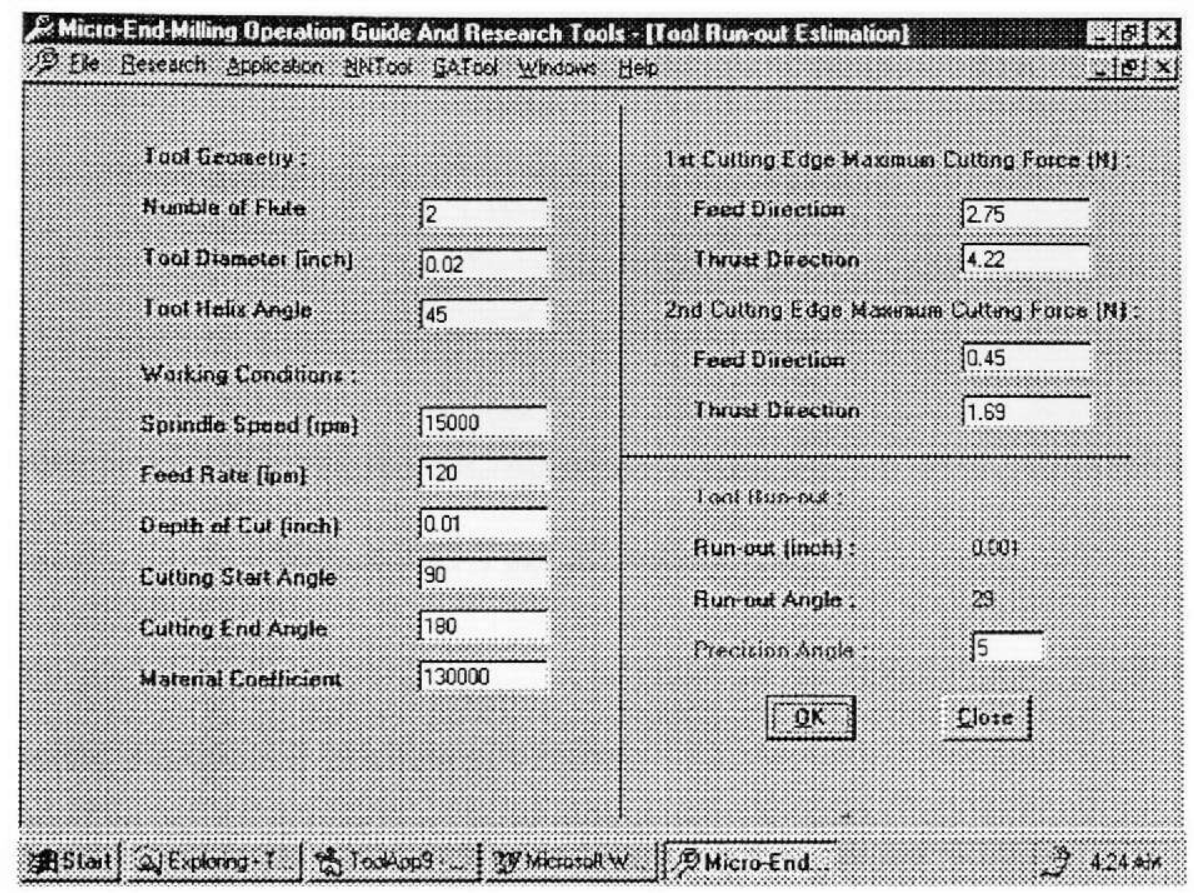

Figure 8.22 Tool run-out estimation

- To estimate the tool life

1. From the Application menu, choose Tool Life Estimation.

2. In the Tool Life Estimation screen (see Figure 8.23), select the parameters one by one, and type the data depending on the tool operation case.

3. Click Ok button. 


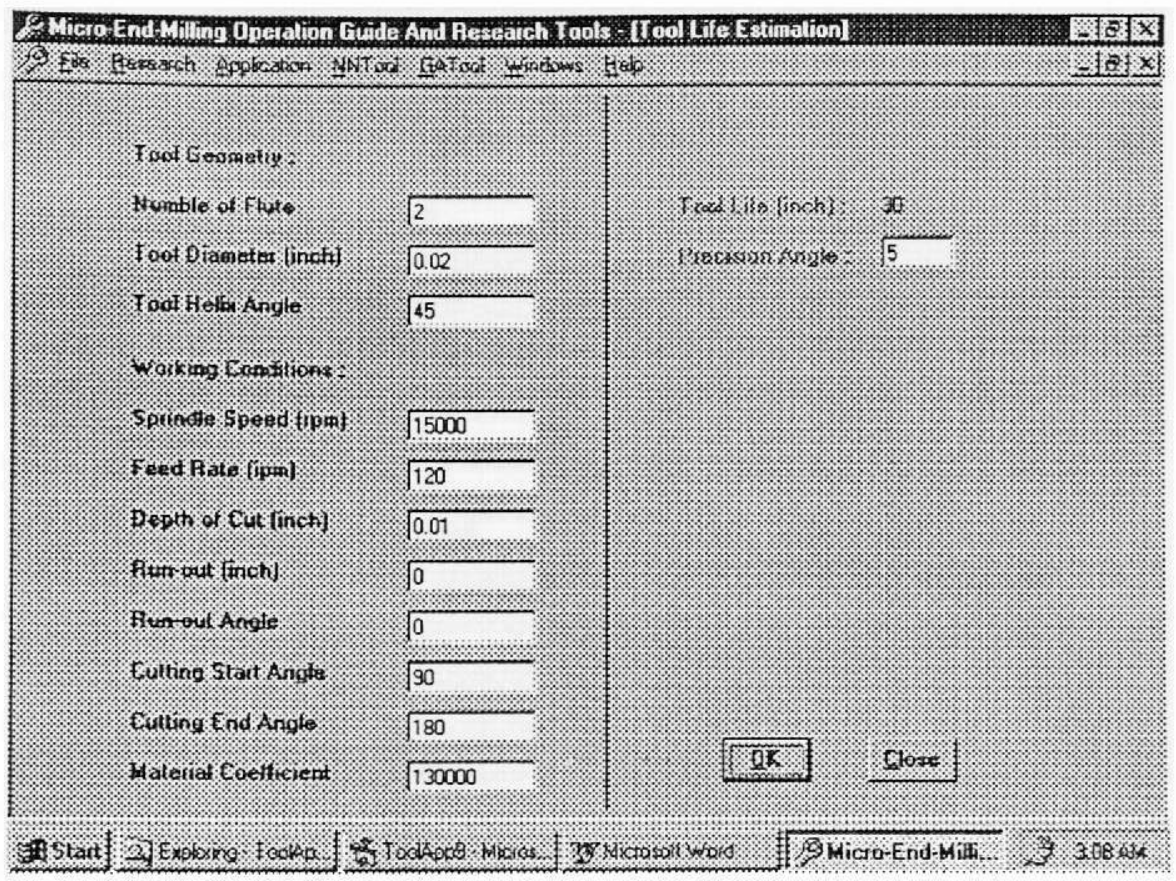

Figure 8.23 Tool life estimation

- To select the optimal working condition

1. From the Application menu, choose Working Condition Selection.

2. In the Working Condition Selection screen (see Figure 8.24), select the parameters one by one, and type the data depending on the tool operation case.

3. In the left section of the screen, point the cursor at the working condition check box that you would like to select, and click the right mouse button.

4. Type the data in the text box of the other working condition parameters.

5. Click Ok button. 


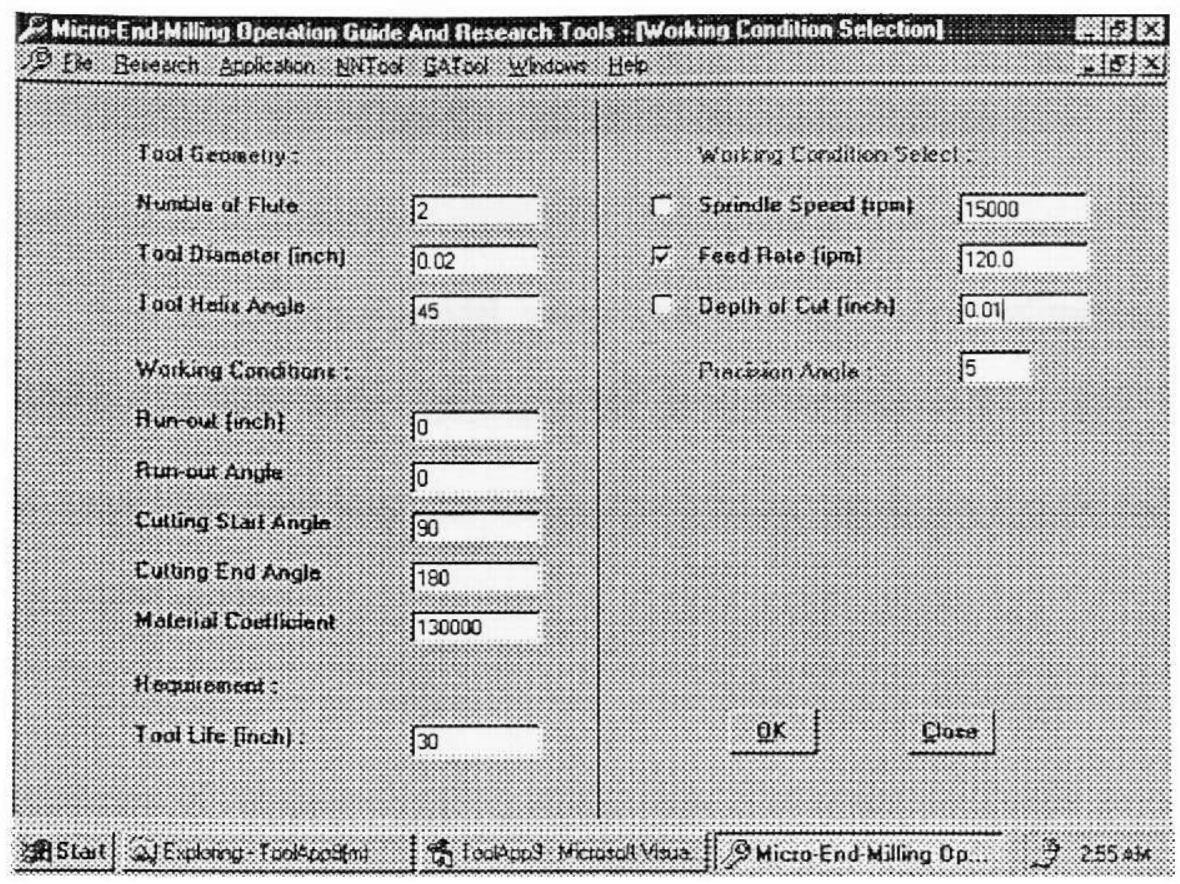

Figure 8.24 Optimal working condition selection 


\section{Chapter IX \\ Conclusion and Recommendations}

A new analytical model was developed for micro-end-milling operations, and computational tools were customized for analysis of experimental data. All the developed tools were integrated with the Micro-End-Milling Operation Guide and Research Tools (MOGART) package.

Studying tool tip trajectory and experimental data developed the analytical cutting force model of micro-end-milling operations. The analytical model estimates the cutting force variation of micro and conventional end milling operation with or without tool runout and wear. Ten parameters and two coefficients are considered in the model. They are three working condition variables (spindle speed, feed rate and depth of cut), two tool run-out variables (run-out and its angle), two cutting condition variables (tool cutting entry and exit angle) and three tool geometry variables (tool diameter, helix angle and the numbers of tool flutes). The material coefficient is related to the tool and work-piece materials. The wear coefficient is related to the tool life. The estimated cutting forces of the analytical model were well matched with the experimental cutting force data. Compared to the numerical procedures, computational time is extremely short. The model is very convenient to study the characteristics of the cutting forces at various different conditions and model based monitoring method is introduced for the first time in this study. 
The MOGART package developed in this study mainly includes three researches:

a) analytical cutting force model, b) neural network based experimental data processing module and c) genetic algorithm based optimization routines. Experimental data can be easily passed to the package through Excel worksheet or through import data file option. Similarly, the results of any analysis can be transferred to a text document and an Excel worksheet based chart .

For machinability study, back-propagation neural network was proposed for twodimension and three-dimension interpolation. The empirical maximum cutting force estimation models and wear forecasting models of the micro-end-milling operations were generated with acceptable errors by using a back-propagation neural network program (NNTool). This program is a part of the MOGART package and has been tested on the experimental data. The tool maximum cutting force and wear characteristics of micro-endmilling operations can be estimated at any cutting condition after the neural network was trained. The program saved a lot of time in machinability studies. The back-propagation neural network was found as an excellent interpolation tool for micro-end-milling and other three-dimension cases.

In the first time, genetic algorithm was used to estimate the parameters of the analytical model to monitor micro-end-milling operations. Wear, breakage, run-out, cutting angles and optimal working conditions were estimated instantaneously without requiring any prior experimental data analysis or training. The proposed approach was found fast and accurate when it was tested on the simulated and experimental data. A genetic algorithm program (GATool) is included in the MOGART package. The 
MOGART was used for the estimation of optimal cutting condition, run-out, wear and breakage. It minimized the cost of data processing and allowed analysis of identical data by using different methods to compare the results.

For different micro-end-mills, work-pieces and working conditions of the microend-milling operations, over 800 milling operation experiments were performed and more than 160 megabytes of cutting force data was collected for this study. The most representative and reliable data were separated and used in this study.

The NNTool and GATool modules were designed for general purpose data processing applications. A modified version of NNTool was used for environmental engineering studies.

The following research work could be done to improve the presented work:

1. Improvement of the analytical model

- Identifying the difference between conventional and micro end milling machining based on the experimental data.

- Investigation of tool wear at different operating conditions to improve the empirical tool wear model.

- Addition of dynamic characteristics of the tools to the model.

2. On line monitoring

- Development of a monitoring system, which would estimate the tool wear and evaluate the cutting conditions to keep the quality of the production at the desired standards. 


\section{LIST OF REFERENCES}

[1] J. Tlusty, P. Macneil, "Dynamics of Cutting Forces In End Milling", Annals of the CIRP, Vol. 24, No. 1, 1975, pp. 21-25.

[2] P. E. Gygax, "Dynamics of Single-Tooth Milling", IWF/ETH Zurich, Annals of the CIRP, Vol. 28, No. 1, 1979, pp. 65-70.

[3] G. Yucesan, A. E. Bayoumi, L. A. Kendall, "An Analytic Cutting Force Model for Milling", Transactions of the North American Manufacturing Research Institution of SME 1990, May 1990, pp. 137-145.

[4] Krzysztof Jemlelniak , "Modelling of Dynamic Cutting Coefficients in ThreeDimensional Cutting", International Journal of Machine Tools \& Manufacture, 1992, Vol. 32, No. 4, pp. 509-519.

[5] J.-J. Junz Wang, S. Y. Liang, W. J. Book, "Convolution Analysis of Milling Force Pulsatio", Journal of Engineering for Industry, February 1994, Vol. 116, pp. 17-25.

[6] Fangming Gu, S. G. Kapoor, R. E. DeVo, “An Approach to On-line Cutter Runout Estimation in Face Milling", Transactions of the North American Manufacturing Research Institution of SME, May 1991, pp. 240-247.

[7] J. W. Sutherland, R. E. DeVor, "An Improved Method for Cutting Force and Surface Error Prediction in Flexible End Milling Systems", Journal of Engineering for Industry, November 1986, Vol. 108, pp. 269-279. 
[8] E. J. A. Armarego, N. P. Deshpande, "Computerized End-Milling Force Predictions with Cutting Models Allowing for Eccentricity and Cutter Deflection", Annals CIRP, Vol. 19, No. 1, 1991, pp. 25-29.

[9] H. S. Kim, K. F. Ehmann, "A Cutting Force Model for Face Milling Operatins", International Journal of Machine Tools \& Manufacture, 1993, Vol. 33, No. 5, pp. 651-673.

[10]Rosenblatt, F., "The Perceptron: A Probabilistic Model for Information Storage and Organization in The Brain", Psychol. Rev., Vol. 65, pp. 386-408, 1958.

[11]Robert Hecht-Nielsen, "Neurocomputing", Addison-Wesley Publishing Company, Inc., 1990.

[12]Russell C. Eberhart, Roy W. Dobbins, "Neural Network PC Tools: A Practical Guide”, Academic Press, Inc., 1990.

[13]Stephen T. Welstead, "Neural Network and Fuzzy Logic Applications In C/C ++", John Wiley \& Sons, Inc., 1997.

[14]W.Y.Bao, I.N.Tansel, T.T.Arkan, B.Tansel, "Visualization of Underground Contamination with A Self Contained Package", Proceedings of the American Society of Civil Engineers, Florida / South Florida Section Conference, September 18-20, 1997.

[15]I.N.Tansel, W.Y.Bao, T.T.Arkan, B.Tansel, "Visualization of Underground Contamination by Using Neural Networks", Smart Engineering System: Neural Networks, Fuzzy Logic, Data Mining, and Evolutionary Programming, Intelligent Engineering 
Systems Through Artificial Neural Networks, Vol.7, pp. 1007-1012, ASME Press, 1997.

[16]I.N.Tansel, W.Y.Bao, T.T.Arkan, B.Shisler, M.McCool, and D.Smith, "Neural Network Based Cutting Force Estimators for Micro-End-Milling Operations", Smart Engineering System: Neural Networks, Fuzzy Logic, Data Mining, and Evolutionary Programming, Intelligent Engineering Systems Through Artificial Neural Networks, Vol.7, pp. 885890, ASME Press, 1997.

[17]I.N.Tansel, W.Y.Bao, B.Tansel, C.M.Jordahl, "Modeling, Contamination Sites with Trainable Networks", Fuzzy Logic and Evolutionary Programming, Intelligent Engineering Systems Through Artificial Neural Networks, Vol.5, pp.823-828, ASME Press, 1995.

[18]Scott Robert Ladd, "Genetic Algorithms in C++", M\&T Books, 1996.

[19]I.N.Tansel, W.Y.Bao, B.Tansel, B.Shisler, D.Smith and J.Murray, "Identification of Cutting Conditions by Using an Analytical Model and Genetic Algorithms for MicroEnd-Milling Operations", Smart Engineering System Design: Neural Networks, Fuzzy Logic, Rough Sets and Evolutionary Programming, Intelligent Engineering Systems Through Artificial Neural Networks, Vol.8, pp. 779-784, ASME Press, 1998.

[20]S. D. J. A. Arsecularatne, G. Barrow, S. Hinduja, "Prediction of The Radial Force in Turing Using Feed Force Data", International Journal of Machine Tools \& Manufacture, 1993, Vol. 33, No. 6, pp. 827-839. 
[21]M. Liu, S. Y. Liang, "Monitoring of Peripheral Milling Using Acoustic Emission", Transactions of the North American Manufacturing Research Institution of SME 1990, May 1990, pp. 120-127.

[22]P. Bandyopadhyay, E. M. Gonzalez, R. Huang, S. M. Wu, "A feasibility Study of on Line Monitoring of DDS Methodology," International Journal of Machine Tool Design \& Research, 1986, Vol. 26, pp. 245-257.

[23]T. I. Liu, "Automated Visual Inspection of drill wear", ASME Manufacturing International Conference, Atlanta, Georgia, 1990.

[24]K. Uehara, "New Attempts for Short time tool life testing", Annals of the CIRP, 1973, Vol. 22, pp. 23.

[25]M. E. Merchant, E. J. Krabacher, "Radioactive Tracers for Rapid Measurements of Cutting Tool Life", Journal of Applied Physics, 1951, Vol. 22, pp. 1507-1508.

[26]J. C. Principle, T. Yoon, “ A New Algorithm for The Detection of Tool Breakage in Milling”, International Journal of Machine Tools \& Manufacture, 1991, Vol. 31, No. 4, pp. $443-454$.

[27]I.N.Tansel, M.Trujillo, A.Nedbouyan, C.Velez, W.Y.Bao, T.T.Arkan and B.Tansel, "Micro-end-milling-III: Wear estimation and tool breakage detection using acoustic emission signals", Machine Tools \& Manufacture, Vol.38, No.12, pp.1449-1466, December 1998.

[28]I.N.Tansel, M.Trujillo, W.Y.Bao, T.T.Arkan, "Detecting Microtool Failures," Cutting Tool Engineering, Vol.49, No.6, pp.54-62, September, 1997. 
[29]I.N.Tansel, M.Trujillo, W.Y.Bao, T.T.Arkan, "Detection of Tool Breakage in MicroEnd-Milling Operations by Monitoring Acoustic Emission", Technical Papers of the North American Manufacturing Research Institution of Society of Manufacturing Engineers (NAMRI SME 1997), pp.69-74, May 20-23, 1997.

[30]I.N.Tansel, M.E.Trujillo, W.Y.Bao, T.T.Arkan, "Tool Breakage Detection in the Micro-Machining of Aluminium by Monitoring Acoustic Emission", Proceedings of the 1997 Florida Conference on Recent Advances in Robotics, pp.230-234, April 1011, 1997.

[31]I.N.Tansel, W.Y.Bao, M.Trujillo, O.Rodriguez, T.T.Arkan, "Detection of Prefailure Phase in Micro-End-Milling operations", presented at the Industrial Engineering Research Conference (IERC Solutions'97), May 17-18, 1997.

[32]S. Y. Liang, D. A. Dornfeld, "Tool Wear Detection Using Time Series Analysis of Acoustic Emission", Joural of Engineering for Industry, August 1989, Vol. 111, pp. 199205.

[33]M. A. Elbestawi, T. A. Papazafiriou, R. X. Du, "In-process Monitoring of Tool Wear in Milling Using Cutting Force Signature", International Journal of Machine Tools \& Manufacture, 1991, Vol. 31, No. 1, pp. 55-73.

[34]K. Glass, R. Colbaugh, "Real-Time Tool Wear Estimation Using Cutting Force Measurements", Interational Conference on Robotics and Automation, Minneapolis, Minnesota, April 1996, pp. 3067-3072. 
[35]M. A. El Baradie, "Statistical Analysis of the Dynamic Cutting Coefficients and Machine Tool Stability", Transactions of The ASME, Journal of Engineering for Industry, May 1993, Vol. 115, pp. 205-215.

[36]I. N. Tansel, C. McLaughlin, "Detection of Tool Breakage in Milling Operations- Time Series Approach", International Journal of Machine Tools Manufacture, Vol. 33, No. 4, 1993, pp. 531-544.

[37]T. J. Ko, D. W. Cho, "Tool Wear Monitoring in Diamond Turning by Fuzzy Pattern Recognition", Transactions of The ASME, Journal of Engineering for Industry, May 1994, Vol. 116, pp. 225-232.

[38]I. N. Tansel, O. Rodriguez, M. Trujillo, E. Paz, W. Li, "Wear Induced Stress (WIS) And Tool Breakage in Micro-End-Milling", Intelligent Engineering Systems Through Artificial Neural Networks, November 1995, Vol. 5, pp. 867-872.

[39]I.N.Tansel, W.Y.Bao, T.T.Arkan and B.Shisler, "Wear Estimation in Micro-End Milling with Wavelet Transformations and Probabilistic Neural Networks", Smart Engineering System Design: Neural Networks, Fuzzy Logic, Rough Sets and Evolutionary Programming, Intelligent Engineering Systems Through Artificial Neural Networks, Vol.8, pp. 755-760, ASME Press, 1998.

[40]I. N. Tansel, C. McLaughlin, "Detection of Tool Breakage in Milling Opeartions - Neural Network Approach", Int. Journal of Machine Tools Manufact., Vol 33, No. 4, 1993, pp. 545-558.

[41]Y. Chao, Y. D. Hwang, "An Improved Neural Network Model for The Prediction of Cutting Tool Life", Journal of Intelligent Manufacturing, 1997, Vol. 8, pp. 107-115. 
[42]I.N.Tansel, W.Y.Bao, T.T.Arkan, B.Shisler, M.McCool, A.Jinks, and D.Smith, "Wear Estimation for Micro-Machining of Non-Metal Materials", Smart Engineering System: Neural Networks, Fuzzy Logic, Data Mining, and Evolutionary Programming, Intelligent Engineering Systems Through Artificial Neural Networks, Vol.7, pp.903-908, ASME Press, 1997.

[43]Milton C. Shaw, "Metal Cutting Principles", Oxford University Press, New York, 1986.

[44]S. M. Pandit, S. M. Wu, "Time Series and System Analysis with Applications", John Wiley and Sons, Inc., 1983.

[45]Timothy Masters, "Advanced Algorithms for Neural Networks", John Wiley \& Sons, Inc., 1995.

[46]B.Tansel, I.N.Tansel, W.Y.Bao, "Neural Network Based performance Estimator for High Energy Electron Beam Irradiation Process", Smart Engineering System Design: Neural Networks, Fuzzy Logic, Rough Sets and Evolutionary Programming, Intelligent Engineering Systems Through Artificial Neural Networks, Vol.8, pp. 839-844, ASME Press, 1998. 
1982

1982-1985

B.S., Power Machinery Engineering Shanghai Jiao Tong University

Shanghai, China

1988

1988-1990
Shanghai Ocean Fishery Company, China

M.S., Power Machinery Engineering

Shanghai Jiao Tong University

Shanghai, China

Shanghai Jiao Tong University, China

\section{PUBLICATIONS AND PRESENTATIONS}

- Published Journal Papers:

1. "Micro-end-milling-III: Wear estimation and tool breakage detection using acoustic emission signals", I.N.Tansel, M.Trujillo, A.Nedbouyan, C.Velez, W.Y.Bao, T.T.Arkan and B.Tansel, "Machine Tools \& Manufacture", Vol.38, No.12, pp.1449-1466, December 1998.

2. “Detecting Microtool Failures," I.N.Tansel, M.Trujillo, W.Y.Bao, T.T.Arkan, "Cutting Tool Engineering", Vol.49, No.6, Vol.8, pp.54-62, September, 1997.

- Refereed Papers Published in Books:

3. "Identification of Cutting Conditions by Using an Analytical Model and Genetic Algorithms for Micro-End-Milling Operations", I.N.Tansel, W.Y.Bao, B.Tansel, B.Shisler, D.Smith and J.Murray, Vol.8, pp. 779-784, 1998.*

4. "Wear Estimation in Micro-End Milling with Wavelet Transformations and Probabilistic Neural Networks", I.N.Tansel, W.Y.Bao, T.T.Arkan and B.Shisler, Vol.8, pp. 755-760, 1998.*

5. "Neural Network Based performance Estimator for High Energy Electron Beam Irradiation Process", B.Tansel, I.N.Tansel, W.Y.Bao, Vol.8, pp. 839-844, 1998.*

* Presented at the Artificial Neural Networks In Engineering (ANNIE'98) Conference and published at the Smart Engineering System Design: Neural Networks, Fuzzy Logic, Rough Sets and Evolutionary Programming, Intelligent Engineering Systems Through Artificial Neural Networks, edited by Drs. Dagli, Akay, Buczak, Ersoy, and Fernandez, ASME Press, New York, 1998. 
6. "Neural Network Based Cutting Force Estimators for Micro-End-Milling Operations", I.N.Tansel, W.Y.Bao, T.T.Arkan, B.Shisler, M.McCool, and D.Smith, Vol.7, pp.885-890, 1997.**

7. "Wear Estimation for Micro-Machining of Non-Metal Materials", I.N.Tansel, W.Y.Bao, T.T.Arkan, B.Shisler, M.McCool, A.Jinks, and D.Smith, Vol.7, pp.903-908, 1997.**

8. "Visualization of Underground Contamination by Using Neural Networks", I.N.Tansel, W.Y.Bao, T.T.Arkan, B.Tansel, Vol.7, pp.1007-1012, 1997.**

** Presented at the Artificial Neural Networks In Engineering (ANNIE'97) Conference and published at the Smart Engineering System: Neural Networks, Fuzzy Logic, Data Mining, and Evolutionary Programming, Intelligent Engineering Systems Through Artificial Neural Networks, edited by C.H.Dagli, M.Akay, O.Ersoy, B.R.Fernandez, and A.Smith, ASME Press, New York, 1997.

9. "Modeling, Contamination Sites with Trainable Networks", I.N.Tansel, W.Y.Bao, B.Tansel, C.M.Jordahl, presented at the Artificial Neural Networks in Engineering (ANNIE'95) Conference and published at the Fuzzy Logic and Evolutionary Programming, Intelligent Engineering Systems Through Artificial Neural Networks, Vol.5, pp.823-828, ASME Press, New York, 1995.

- Published Conference Papers:

10. "Visualization of Underground Contamination with A Self Contained Package", W.Y.Bao, I.N.Tansel, T.T.Arkan, B.Tansel, Proceedings of the American Society of Civil Engineers, Florida / South Florida Section Conference, September 18-20, 1997.

11. "Detection of Tool Breakage in Micro-End-Milling Operations by Monitoring Acoustic Emission", I.N.Tansel, M.Trujillo, W.Y.Bao, T.T.Arkan, Technical Papers of the North American Manufacturing Research Institution of Society of Manufacturing Engineers (NAMRI SME 1997), pp.69-74, May 20-23, 1997.

12. "Detection of Prefailure Phase in Micro-End-Milling Operations", I.N.Tansel, W.Y.Bao, M.Trujillo, O.Rodriguez, T.T.Arkan, presented at the Industrial Engineering Research Conference (IERC Solutions'97), May 17-18, 1997.

13. "Tool Breakage Detection in the Micro-Machining of Aluminium by Monitoring Acoustic Emission", I.N.Tansel, M.E.Trujillo, W.Y.Bao, T.T.Arkan, Proceedings of the 1997 Florida Conference on Recent Advances in Robotics, pp.230-234, April 10$11,1997$.

14. "Prototyping for High-Tech Consumer Products", B.Shisler, D.Johnson, D.Smith, I.N.Tansel, W.Y.Bao, T.T.Arkan, Proceedings of the 1997 Florida Conference on Recent Advances in Robotics, pp.24-26, April 10-11, 1997.

15. "Design and Realization of an Automated Log Strip Seperator", I.N.Tansel, T.T.Arkan, W.Y.Bao, J.Shaw, C.A.Velez, T.C.Yih, S.Tosunoglu, I.Fernandez, B.Tansel, Proceedings of the 1997 Florida Conference on Recent Advances in Robotics, pp.8-11, April 10-11, 1997.

16. "Solving industrial Problems in Mechatronics Class", I.N.Tansel, T.T.Arkan, W.Y.Bao, J.Show, C.A.Veldez, T.C.Yih, S.Tosunoglu, I.Fernandez, Proceedings of Mechatronics 96, June 13-15, 1996. 